\title{
Endocrine and behavioural plasticity in vertebrates
}

\author{
Submitted by \\ Amanda Louise Fry
}

Submitted to Swansea University in fulfilment of the requirements for the Degree of Doctor of Philosophy

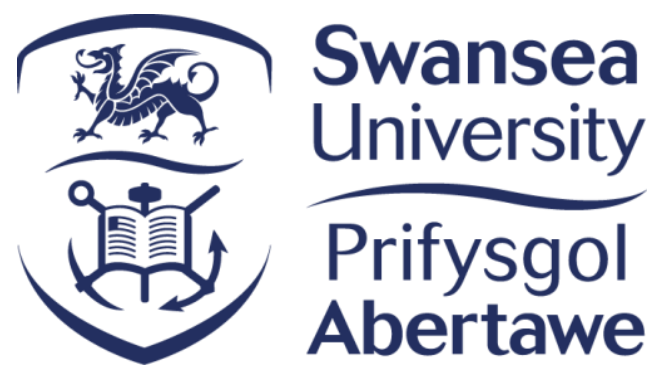

Swansea University, 2021 


\begin{abstract}
Endocrine plasticity is the reversible change in endocrine traits in response to unpredicted changes in an animals' physical and social environment. Inter-individual differences in how individuals' endocrine traits vary can occur as a result of differences in how individuals' hypothalamic-pituitary-adrenal/interrenal (HPA/I axis) respond to stressors. However, it remains unknown if and how the variation in individuals' baseline stress hormones (measured by glucocorticoids) can predict stress-induced plasticity. In this thesis, I aim to gain a more in-depth understanding of variation in endocrine plasticity over a range of physical and social environmental changes across different vertebrate systems, and the potential factors (behavioural and morphological) driving it. First, I review the literature on between-individual variation in endocrine plasticity to better understand why and how plasticity occurs (Chapter 1) and provide an overview of my methods and study systems (Chapter 2). Second, I investigate the effects of familiarity and recent social context on cortisol responses (Chapter 3) and behavioural responses (Chapter 4) in three-spined stickleback fish (Gasterosteus aculeatus). Third I investigate endocrine plasticity in humans (Homo sapiens) and potential links to BIG 5 personality measures in a social task (Chapter 5). Fourth, I investigate long-term plasticity in Welsh mountain ewes (Ovis aries) in response to changing food availability (Chapter 6). Across these three different study systems I find i) endocrine repeatability, which can be considered equivalent to personality traits, ii) evidence for endocrine plasticity and between-individual differences in plasticity, and iii) links between endocrine repeatability and plasticity to between variations behavioural/morphological traits. In the final chapter (Chapter 7) I discuss how these findings advance our understanding of how individuals respond and adapt to environmental challenges.
\end{abstract}




\section{Contents}

Declaration and Statements ................................................................................4

Acknowledgements............................................................................................................ 5

Statement of Contributions ...................................................................................................... 6

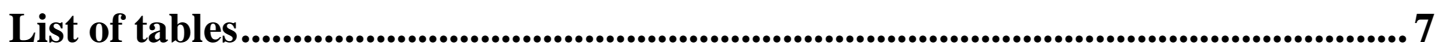

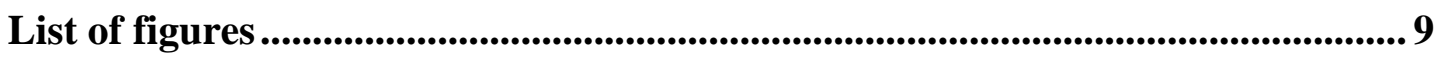

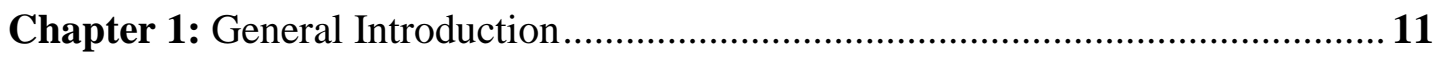

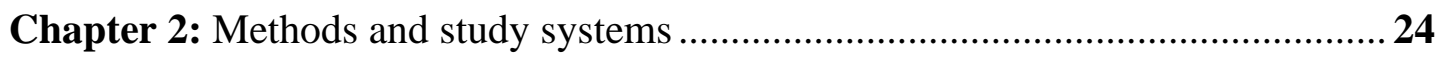

Chapter 3: Effects of recent social context and familiarity on cortisol responses in three-spined stickleback fish (Gasterosteus aculeatus)

Chapter 4: Effects of recent housing condition, familiarity and hypothalamicpituitary-interrenal (HPI) axis activity on behavioural responses in dyadic social contexts in three-spined stickleback fish (Gasterosteus aculeatus)..........................50

Chapter 5: Repeatability and plasticity in human endocrine traits 66

Chapter 6: Repeatability and plasticity in sheep wool cortisol in response to changing food availability .87

Chapter 7: General Discussion .......................................................................... 105

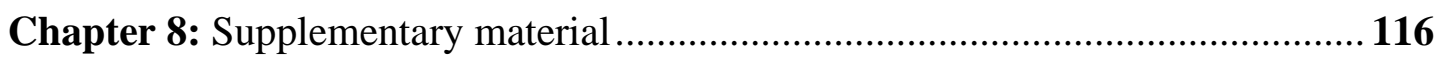

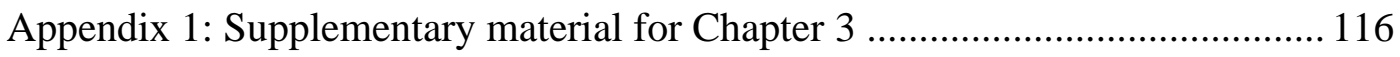

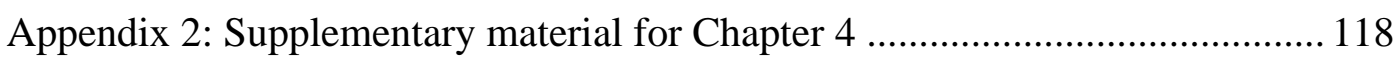

Appendix 3: Supplementary material for Chapter 5 .......................................... 120

Appendix 4: ASAB Conference Poster ............................................................. 126

Appendix 5: ECBB Conference Poster .......................................................... 127

Chapter 9: References..................................................................................... 128 


\section{Declaration and Statements}

\section{DECLARATION}

This work has not previously been accepted in substance for any degree and is not being concurrently submitted in candidature for any degree.

Signed

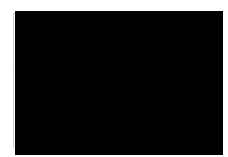

(candidate)

Date: $31 / 03 / 2021$

\section{STATEMENT 1}

This thesis is the result of my own investigations, except where otherwise stated. Where correction services have been used, the extent and nature of the correction is clearly marked in a footnote(s).

Other sources are acknowledged by footnotes giving explicit references. A bibliography is appended.

Signed

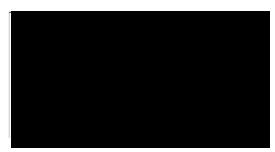

(candidate)

Date: $31 / 03 / 2021$

\section{STATEMENT 2}

I hereby give consent for my thesis, if accepted, to be available for photocopying and for inter-library loan, and for the title and summary to be made available to outside organisations.

Signed (candidate)

Date: $31 / 03 / 2021$ 


\section{Acknowledgements}

I would not have made through my $\mathrm{PhD}$ without the help and support of a range of people. Firstly, I would like to thank my supervisors who have provided tremendous amount of help throughout my $\mathrm{PhD}$ and have helped me grow as a research scientist. My first supervisor Ines Fürtbauer has provided invaluable behavioural endocrinology knowledge, taught me numerous laboratory skills, scientific writing advice and presentation skills; with her help I have grown as a scientist. My second supervisor Andrew King has provided invaluable advice on experimental design, great explanations and help with scientific writing and good practices. Outside office hours they have arrange many social sessions which fostered a great lab group community, including our "Staying Inn" virtual pub during lockdown!

I would like to thank my friends that I have met during my $\mathrm{PhD}$ journey. I would like to thank Charlotte Solman for all her scientific advice, reading through my drafts, for listening to all my rambling and being there for a chat about anything when I needed. I would also like to thank Charlotte Christensen and Anna Bracken for our many games nights and answers to my random questions on our group chat, you three have helped me keep sane! Lastly, I would like to thank Dr Rebecca Pawluk, for being such a great friend throughout the years, helping me with all my $\mathrm{PhD}$ related questions and for our venting tea sessions.

Most of all, I would like to thank my amazing fiancé James Lewis for all his emotional support and for keeping me motivated throughout these last four years. You have been my rock, supporting me through all my ups and downs and I could not have made it through without you. Thank you for all your love, care and support you have given me throughout, especially this last year. 


\section{Statement of Contributions}

Table 1.0: List of contributions of myself (AF, the candidate), Dr Ines Fürtbauer (IF, first supervisor) and Dr Andrew King (AK, second supervisor) towards each element of work for thesis data chapters (3-6).

\begin{tabular}{lllll}
\hline Contributor & \multicolumn{5}{c}{ Chapter } \\
\cline { 2 - 5 } Role & \multicolumn{1}{c}{$\mathbf{3}$} & \multicolumn{4}{c}{$\mathbf{4}$} & \multicolumn{1}{c}{$\mathbf{6}$} \\
\hline Conceptualisation & AF, IF & AF, IF, AK & AF, IF, AK & AF, IF \\
Data Collection & AF & AF & AF & IF \\
Hormone analysis & AF & AF & AF & AF \\
Laboratory & IF & IF & IF & IF \\
supervision & & & & \\
Data Analysis & AF & AF & AF & AF \\
$\begin{array}{l}\text { Data visualisation } \\
\text { Writing- Original }\end{array}$ & AF & AF & AF & AF \\
$\begin{array}{l}\text { Draft } \\
\text { Writing- Review }\end{array}$ & AF, IF, AK & AF, IF, AK & AF, IF, AK & AF, IF, AK \\
\& Editing & & & & \\
\hline
\end{tabular}




\section{List of tables}

Table 1.1: Some examples of phenotypic plasticity in morphology, behaviour and physiology across species and contexts, with known or predicted adaptive reason for plastic response. 14

Table 1.2: Summary of data chapter (3-6), research questions (ticks indicate whether a question is addressed in a data chapter), and sample type for hormone analysis.... 22 Table 3.1: Changes in waterborne cortisol concentrations (ng/g/h) across pre-NE and post-NE measures for $\mathrm{n}=72$ stickleback fish. Fish ID and Dyad ID were included as random effects. Statistically significant results are shown in bold.

Table 3.2: Factors affecting pre-NE (LMM2) and post-NE (LMM3) waterborne cortisol concentrations $(\mathrm{ng} / \mathrm{g} / \mathrm{h})$ for $\mathrm{n}=72$ stickleback fish. Fish ID and Dyad ID were included as random effects. Statistically significant results are shown in bold. 43 Table 3.3: Summary of LMMs exploring potential cortisol coregulation in stickleback fish dyads ( $\mathrm{n}=36$ dyads) for pre- and post-NE cortisol measurements. Note, both cortisol and partner cortisol were log transformed (see methods for details). Fish ID and Dyad ID were included as random effects. Statistically significant results are shown in bold.

Table 4.1: Variables included in final models investigating repeatability, predictors of behaviour and potential changes in the four behaviour measures across two trials. Random effect(s) were included in all (G)LMMs to control for the non-independence of data. Sex was controlled for in individual behaviour models.

Table 4.2: Post hoc analysis of proportion of individual time in shelter with partner (\%) for each familiarity context; familiar and unfamiliar dyads, $\mathrm{n}=36$ dyads. Statistically significant results are shown in bold.

Table 5.1: Summary information for sex, age, salivary cortisol and testosterone concentrations and PSS scores, averaged across two sampling points for $n=13$ male and female participants. PSS is scored on a scale of 0-40, generally a score of 0-13 is considered 'low stress' and a score of 27-40 is considered 'high stress'. Note that a complete table with all measures (pre- and post-hormone concentrations, PSS and scores for each personality trait) for each participant is presented in Appendix 3 Table 8.7). .75

Table 5.2: Factors predicting individual cortisol concentrations $(\mu \mathrm{g} / \mathrm{dL})$ and testosterone concentrations $(\mathrm{pg} / \mathrm{mL})$ before and after a social task in female and male participants $(n=13)$. Participant ID was included as a random effect. Statistically significant results are shown in bold. .76

Table 5.3: Factors predicting perceived stress in female and male participants $(n=13)$. Participants ID was included as a random effect. Significant results are shown in bold.

Table 5.4: Correlations between Big 5 personality traits and average cortisol (Spearman correlations) and testosterone concentrations (Pearson correlations) including p-values in brackets. Statistically significant results are shown in bold.... 81 Table 6.1: Factors predicting individual body mass $(\mathrm{kg})$ in $n=33$ sheep. Sheep ID was included as a random effect. Statistically significant results are shown in bold. 96 
Table 6.2: Factors predicting individual wool cortisol concentrations $(\mu \mathrm{g} / \mathrm{dL})$ in $n=33$ sheep. Sheep ID was included as a random effect in both models. Statistically significant results are shown in bold. 97

Table 6.3: Comparison of LMMs with different fixed and random effects using an ANOVA test to compare log-likelihood ratios ( $n=120$ cortisol measures). Grazing period was included as a fixed effect in each model. Statistically significant results are shown in bold. 98

Table 6.4: Factors predicting cortisol change (LMM9) and adjusted cortisol change (LMM10) during two grazing periods in $n=33$ sheep. Sheep ID was included as a random effect in both models. Statistically significant results are shown in bold..... 98 Table 7.1: Summary of research questions addressed in each data chapter. Green ticks indicate evidence, red crosses indicate absence of evidence (empty box indicates research question not addressed). 106

Table 8.1: Summary of LMMs exploring potential cortisol coregulation in stickleback fish dyads ( $\mathrm{n}=36$ dyads) for post-NE cortisol measurements, and potential interactions between recent social context and familiarity. Note, both cortisol and partner cortisol were $\log$ transformed (see methods for details). Initially, the model suggested an interaction between housing and partner cortisol (LMM6), but when the nonsignificant familiarity and partner cortisol interaction was removed the interaction was no longer significant (LMM7). Fish ID and Dyad ID were included as random effects. Statistically significant results are shown in bold. 116

Table 8.2: Summary of effect of individual and partner cortisol concentration on changes in cortisol concentrations from pre-NE to post-NE1. Note pre-NE and partner pre-NE cortisol was logged transformed. Fish ID and Dyad ID were included as random effects. Statistically significant results are shown in bold. 116

Table 8.3: Model output of post hoc analysis ran to investigate whether housing individual socially or solitarily affected change in cortisol. Cortisol change (log transformed) was the response and an interaction between pre-NE cortisol and housing was included. Group ID was included as a random effect.

Table 8.4: Repeatability of (i) total distance travelled, (ii) individual shelter use and (iii) proportion of individual time in shelter with partner between trial one and trial two, controlling for sex, $n=72$ stickleback fish. Dyad ID was included as a random effect. Statistically significant results are shown in bold.

Table 8.5: Repeatability of inter-dyad distance travelled between trial one and trial two, $\mathrm{n}=36$ stickleback fish dyads. 118

Table 8.6: Factors affecting total distance travelled (m), individual shelter time (mins), proportion of individual time in shelter with partner $(\%)$ and inter-dyad distance (m) across two behaviour trial experiments ( $n=72$ individuals; $n=36$ dyads). Fish ID and Dyad ID was included as random effects where appropriate. Statistically significant results are shown in bold.

Table 8.7: Complete table with all measures (pre- and post-hormone concentrations, PSS and scores for each personality trait) for each participant, $n=13$. 123 


\section{List of figures}

Figure 1.1: Theoretical reaction norm scenarios of phenotypes within a population in response to an environmental gradient (e.g. change in food availability). Each line represents an individual within a population in response to an environmental gradient where, a) shows individual differences in phenotypes but no plasticity, b) shows individuals have same phenotype and plasticity, c) shows individual differences but same plasticity direction and d) shows individual differences in phenotype and plasticity (adapted from Dingemanse et al. 2010). .............................................. 12

Figure 2.1: Overview of the time from hormone secretion to appearance in noninvasive sample types (adapted from Behringer \& Deschner 2017). 25

Figure 2.2: Schematic diagram of waterborne hormone sampling, extraction and analysis methodology.

Figure 2.3: Schematic diagram for methodology of wool collection and cortisol extraction and analysis.

Figure 2.4: Study systems used in my thesis; A) Three-spined stickleback fish, B) Humans and C) Welsh mountain ewes.

Figure 3.1: Experimental setup for investigating the effects of recent social context and familiarity on cortisol responses in three-spined stickleback fish. (A) Individuals housed solitarily prior to experiments and tested with an unfamiliar partner (that had also been housed solitarily). (B) Individuals housed socially and tested with an unfamiliar partner. (C) Individuals housed socially and tested with that familiar partner. Dyads were tested in the novel environment tank twice and a post-test hormone sample was taken each time, a pre-test sample was also taken before the experiments ( $n=3$ samples per individual; see also Figure 3.2). Dyad partners remained the same throughout the experiments.

Figure 3.2: Timeline of experiment and waterborne hormone collection. Fish dyads were exposed twice to the novel environment. $\mathrm{N}=3$ waterborne hormone samples (total $\mathrm{n}=216$ ) were collected from each individual (one pre- and two post-test samples). . 40 Figure 3.3: Waterborne cortisol concentrations in $n=72$ stickleback fish. For each fish, samples were collected before (pre-NE) and after the first (post-NE1) and second (post-NE2) novel environment test. Note that untransformed cortisol concentrations are shown. Box and whiskers show average value, first and third quartile and minimum and maximum values. Data points that fall outside the quartiles are outliers represented by dots.

Figure 3.4: Correlation between (A) partner and (B) individual Pre-NE cortisol concentrations $(\mathrm{ng} / \mathrm{g} / \mathrm{h})$ and the change in cortisol concentrations between Pre-NE and the first novel environment test (post-NE1), $\mathrm{n}=72$.

Figure 3.5: Correlation between individual Pre-NE cortisol concentrations ( $\mathrm{ng} / \mathrm{g} / \mathrm{h}$ ) and the adjusted for regression effects change in cortisol concentrations between Pre$\mathrm{NE}$ and the first novel environment test (post-NE1), $\mathrm{n}=72$.

Figure 4.1: Repeatability in (A) total distance travelled (m), (B) individual shelter use (mins), (C) proportion of individual time in shelter with partner (\%) and (D) inter-dyad distance across two trials ( $\mathrm{T} 1$ and $\mathrm{T} 2 ; \mathrm{n}=72$ individuals; $\mathrm{n}=36$ dyads).

Figure 4.2: Differences in (A) total distance travelled, (B) individual shelter use, (C) proportion of individual time in shelter with partner ( $n=72$ individuals) and (D) inter- 
dyad distance between familiar and unfamiliar stickleback dyads ( $\mathrm{n}=36$ dyads). Box and whisker plots show (for each behaviour measure) average value, first and third quartile values and minimum and maximum values.

Figure 4.3: Relationship between individuals' average waterborne cortisol (ng/g/h) and total distance travelled $(\mathrm{m}), \mathrm{n}=72$ individuals. The blue line represents the predicted effect of average cortisol and distance travelled, with the shaded area representing the $95 \%$ confidence intervals.

Figure 5.1: (A-B) Repeatability in (A) cortisol and (B) testosterone across two sampling points (before and after a social task; $n=13$ ). (C-D) Reaction norm plots showing change ('plasticity') in (C) cortisol and (D) testosterone concentrations.... 77 Figure 5.2: Relationship between pre-task cortisol concentrations and the change in cortisol between the two sampling points $(n=13)$. .78

Figure 5.3: (A) Repeatability and (B) plasticity in perceived stress scores (PSS) across two sampling points (before and after a social task, $n=13$ ).

Figure 5.4: Relationship between average PSS and average cortisol in (A) the original data set $(n=13)$ and $(B)$ the additional data set $(n=56)$ and the relationship between average PSS and percentage change in (C) the original data set and (D) the additional data set.

Figure 6.1: Experimental timeline showing the two grazing periods. At the start of each grazing period, a wool sample was taken, and body mass was measured. Sheep were then allowed to graze freely in an upland pasture. At the end of the grazing period another wool sample and body mass measure were obtained. The second grazing period occurred three months later, and the sampling procedure mirrored the first grazing period.

Figure 6.2: (A) Pre- and post-grazing body mass, (B) pre- and post-grazing WCC ( $\mathrm{pg} / \mathrm{mg}$ ) in two grazing periods, (C) relationship between individuals' body mass $(\mathrm{kg})$ and WCC (pg/mg), (D) differences in WCC between two age groups (3-4 and 5-6 years) and (E) relationship between pre-grazing cortisol (pg/mg) and wool cortisol change during the two grazing periods, $n=33$ sheep. Boxes $(A, B, D)$ indicate average value, first and third quartile values, whiskers indicate minimum and maximum values, and dots represent outliers.

Figure 6.3: Relationship between pre-grazing cortisol (pg/mg) and adjusted wool cortisol change during the two grazing periods, $n=33$ sheep. 100 


\section{Chapter 1: General Introduction}

\section{Phenotypic plasticity}

What is phenotypic plasticity? Phenotypic plasticity is defined as the ability of an organism to modify its phenotype in response to an environmental change, traditionally defined as the ability of a genotype to produce different phenotypes under varying environmental conditions (DeWitt et al. 1998; Miner et al. 2005; Gianoli \& Valladares 2012; Forsman 2015). Phenotypic plasticity can be, broadly split into i) developmental plasticity, i.e. irreversible phenotypic-like modification on development and growth, and ii) phenotypic flexibility, i.e. reversible intra-individual plasticity in physiology, behaviour, morphological and life-history traits (Sih 2004; Forsman 2015). In this thesis, I study phenotypic plasticity (or flexibility) that can occur where individuals experience variable and unpredictable environmental conditions (Windig et al. 2004). This includes changes in the physical environment, (e.g. change of habitat/environment) or changes to the social environment (e,g, changes to group composition; Blumstein 2012; Colson et al. 2012; Miranda-de la Lama et al. 2012; Lowry et al. 2013).

How can we measure phenotypic plasticity? In order to measure phenotypic plasticity, at least two measures of the trait of interest need to be taken (Taff et al. 2018), allowing the use of a 'reaction norm approach' for investigations of individual phenotypic expression across changing environmental conditions (for reviews see Dingemanse et al. 2010; Taff \& Vitousek 2016). Reaction norms describe individual flexibility to environmental changes by analysing the elevation (the phenotype measure, i.e. the intercept which defines the physiological/behaviour/morphological trait being analysed at a specific point) and the slope (measure of plasticity) of individual responses to an environmental gradient (e.g. change in food availability or pre- and post-novel environment; Nussey et al. 2007; Dingemanse et al. 2010; Dingemanse \& Wolf 2013; Ergon \& Ergon 2015). When using the reaction norm approach to measure phenotypic plasticity there are various theoretical scenarios which show different ways individuals may respond to environmental changes (see Figure 1.1 below; Dingemanse et al. 2010). For example, there may be individual differences in the phenotypic trait being measured and no plasticity (i.e. no change 
when the environmental gradient changes; Figure 1.1a), no individual differences in the phenotypic traits and population level plasticity (i.e. all individuals show the same response to the phenotypic trait and environmental change; Figure 1.1b), individual differences in phenotypic trait but all show the same direction of plasticity (Figure 1.1c). Additionally, there may be individual differences in the phenotypic trait and plasticity in response to the environmental gradient (Figure 1.1d).

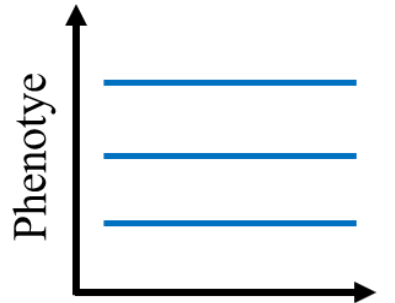

a)

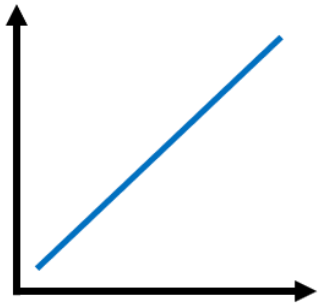

b)

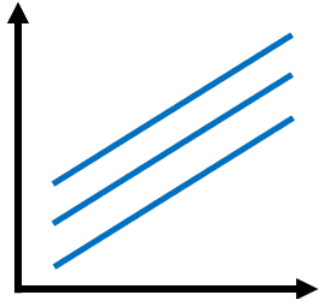

c)

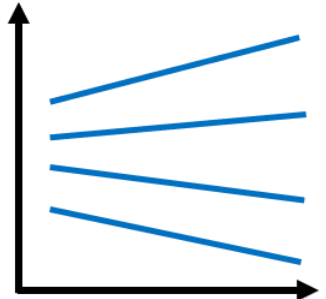

d)

Environmental gradient

Figure 1.1: Theoretical reaction norm scenarios of phenotypes within a population in response to an environmental gradient (e.g. change in food availability). Each line represents an individual within a population in response to an environmental gradient where, a) shows individual differences in phenotypes but no plasticity, b) shows individuals have same phenotype and plasticity, c) shows individual differences but same plasticity direction and d) shows individual differences in phenotype and plasticity (adapted from Dingemanse et al. 2010).

What is the function of phenotypic plasticity? Phenotypic plasticity enables individuals to adaptively change their phenotype in response to changes in their environment, therefore providing direct fitness benefits (Agrawal 2001; Gianoli \& Valladares 2012). Phenotypic plasticity can be short- or long-term depending on the environmental gradient (DeWitt \& Scheiner 2004). Short-term phenotypic plasticity occurs when an individual is exposed to a brief (i.e. minutes or hours) environmental change (Noh et al. 2017). This could include short-term exposure to a novel environment, handling of an individual, brief social isolation (i.e. for a few hours) or being placed with an unfamiliar individual for a brief time (i.e. 30 minutes; Thomas $\&$ Simmons 2011; Noh et al. 2017). Long-term phenotypic plasticity, in contrast, occurs when exposure to environmental change is longer (i.e. days, weeks or months); this could include long-term isolation or effects of changes in food availability on body mass (DeWitt \& Scheiner 2004; Noh et al. 2017). Table 1.1 provides some examples 
of plasticity in morphology, behaviour, and physiology across various species and contexts, along with the known or predicted adaptive benefit. In this thesis, I focus upon the final category - plasticity in physiology - and more specifically endocrine plasticity, with a strong focus on the physiological stress response, and also explore the potential links to associated changes in behaviour (e.g. activity, boldness and personality, i.e. coping styles, reviewed by Koolhaas et al. 1999; Coppens et al. 2010) and morphology (e.g. body weight Fokidis et al. 2012; Chapman et al. 2015). 
Table 1.1: Some examples of phenotypic plasticity in morphology, behaviour and physiology across species and contexts, with known or predicted adaptive reason for plastic response.

\begin{tabular}{|c|c|c|c|c|c|}
\hline $\begin{array}{l}\text { Type of } \\
\text { phenotypic } \\
\text { plasticity }\end{array}$ & Context & $\begin{array}{l}\text { Plasticity } \\
\text { response }\end{array}$ & Species & Adaptive reason & References \\
\hline Morphology & $\begin{array}{l}\text { Limiting food } \\
\text { availability }\end{array}$ & $\begin{array}{l}\text { Body weight } \\
\text { decreases }\end{array}$ & Ovis aries & $\begin{array}{l}\text { Decreasing food intake, means there } \\
\text { is less energy available for } \\
\text { metabolic process. As a result, the } \\
\text { body breaks down excess fat stores } \\
\text { and over time body mass decreases. }\end{array}$ & $\begin{array}{l}\text { (Abouheif } \text { et al. 2013; } \\
\text { Swelum } \text { et al. 2017) }\end{array}$ \\
\hline Behaviour & $\begin{array}{l}\text { New } \\
\text { environment }\end{array}$ & $\begin{array}{l}\text { Activity } \\
\text { decreases }\end{array}$ & $\begin{array}{l}\text { Salmo trutta, } \\
\text { Ovis aries }\end{array}$ & $\begin{array}{l}\text { New environments could be } \\
\text { potentially dangerous, i.e. predators, } \\
\text { therefore, it is beneficial for } \\
\text { individuals to move less so they are } \\
\text { less conspicuous to predators. }\end{array}$ & $\begin{array}{l}\text { (Brelin et al. } 2005 \text {; } \\
\text { Miranda-de la Lama et } \\
\text { al. 2012) }\end{array}$ \\
\hline Behaviour & $\begin{array}{l}\text { Familiar } \\
\text { individuals }\end{array}$ & $\begin{array}{l}\text { Increase } \\
\text { exploration }\end{array}$ & $\begin{array}{l}\text { Pimephales promelas, } \\
\text { Plethodon cinereus, } \\
\text { Oreochromis mossambicus }\end{array}$ & $\begin{array}{l}\text { Familiar individual provides a safe } \\
\text { and secure social environment, } \\
\text { where an individual can explore } \\
\text { more. }\end{array}$ & $\begin{array}{l}\text { (Chivers et al. } 1995 ; \\
\text { Liebgold \& Dibble 2011; } \\
\text { Galhardo } \text { et al. 2012) }\end{array}$ \\
\hline Physiology & $\begin{array}{l}\text { New } \\
\text { environment }\end{array}$ & $\begin{array}{l}\text { Increase in } \\
\text { glucocorticoids }\end{array}$ & $\begin{array}{l}\text { Cavia porcellus, } \\
\text { Sus scrofa, } \\
\text { Ovis aries }\end{array}$ & $\begin{array}{l}\text { Short-term increase in } \\
\text { glucocorticoids can heighten } \\
\text { responses and enable individuals to } \\
\text { be alert in a new environment. }\end{array}$ & $\begin{array}{l}\text { (Hennessy et al. 2006; } \\
\text { Colson et al. 2012; } \\
\text { Miranda-de la Lama et } \\
\text { al. 2012) }\end{array}$ \\
\hline Physiology & $\begin{array}{l}\text { Limiting food } \\
\text { availability }\end{array}$ & $\begin{array}{l}\text { Increase in } \\
\text { glucocorticoids }\end{array}$ & $\begin{array}{l}\text { Oncorhynchus mykiss, } \\
\text { Procolobus rufomitratus, } \\
\text { Lemur catta } \\
\text { Rissa tridactylda, } \\
\text { Melospiza melodia, }\end{array}$ & $\begin{array}{l}\text { Decreasing food intake, means there } \\
\text { is less energy available for } \\
\text { metabolic process. Increasing } \\
\text { glucocorticoids can cause a } \\
\text { redirection of energy from non- }\end{array}$ & $\begin{array}{l}\text { (Blom et al. 2000; } \\
\text { Wingfield \& Kitaysky } \\
\text { 2002; Clinchy et al. } \\
\text { 2004; Kitaysky et al. } \\
\text { 2007; Behie et al. 2010; }\end{array}$ \\
\hline
\end{tabular}




\begin{tabular}{|c|c|c|c|c|c|}
\hline & & & $\begin{array}{l}\text { Uria aalge, } \\
\text { Alouatta pigra } \\
\text { Eudocimus albus }\end{array}$ & $\begin{array}{l}\text { essential to essential metabolic } \\
\text { processes. }\end{array}$ & $\begin{array}{l}\text { Herring et al. } 2011 \text {; } \\
\text { Chapman et al. } 2015 \text {; } \\
\text { Fardi et al. 2018) }\end{array}$ \\
\hline Physiology & Handling & $\begin{array}{l}\text { Increase in } \\
\text { glucocorticoids }\end{array}$ & $\begin{array}{l}\text { Scorpis violaceus, } \\
\text { Hemigymmus melapterus, } \\
\text { Oncorhynchus mykiss, } \\
\text { Pelecanoides urinatrix, } \\
\text { Phylloscopus trochilus, } \\
\text { Aptenodytes forsteri, } \\
\text { Turdus merula, } \\
\text { Tachycineta bicolor, } \\
\text { Platymantis vitiana, } \\
\text { Tamias amoenu }\end{array}$ & $\begin{array}{l}\text { Handling an individual is an } \\
\text { unknown potentially unsafe } \\
\text { environment. Therefore, an increase } \\
\text { in glucocorticoids allows for } \\
\text { allocation of resources to the 'fight } \\
\text { or flight' response and heighten } \\
\text { alertness. }\end{array}$ & $\begin{array}{l}\text { (Pankhurst et al. 1992; } \\
\text { Smith } \text { et al. } 1994 ; \\
\text { Silverin } \text { et al. } 1997 ; \\
\text { Grutter \& Pankhurst } \\
\text { 2000; Kenagy \& Place } \\
\text { 2000; Meka \& } \\
\text { McCormick 2005; } \\
\text { Cockrem et al. 2008; } \\
\text { Narayan } \text { et al. 2010; } \\
\text { Adams et al. 2011; } \\
\text { Zimmer 2019) }\end{array}$ \\
\hline Physiology & $\begin{array}{l}\text { Social } \\
\text { isolation }\end{array}$ & $\begin{array}{l}\text { Increase in } \\
\text { glucocorticoids }\end{array}$ & $\begin{array}{l}\text { Callithrix kuhli, } \\
\text { Microtus ochrogaster, } \\
\text { Taeniopygia guttata, } \\
\text { Rattus norvegicus, }\end{array}$ & $\begin{array}{l}\text { For social individuals, social } \\
\text { isolation is an unfamiliar new } \\
\text { environment (see 'new } \\
\text { environment' adaptive reason } \\
\text { above). }\end{array}$ & $\begin{array}{l}\text { (Smith \& French 1997; } \\
\text { Dronjak et al. 2004; } \\
\text { Ruscio et al. 2007; } \\
\text { Banerjee \& Adkins- } \\
\text { Regan 2011; Ferland \& } \\
\text { Schrader 2011) }\end{array}$ \\
\hline Physiology & $\begin{array}{l}\text { Unfamiliar } \\
\text { individuals }\end{array}$ & $\begin{array}{l}\text { Increase in } \\
\text { glucocorticoids }\end{array}$ & $\begin{array}{l}\text { Phodopus sungorus, } \\
\text { Sceloporus jarrovi }\end{array}$ & $\begin{array}{l}\text { An unknown individual could be a } \\
\text { potential threat, and therefore an } \\
\text { increase in glucocorticoids enables } \\
\text { an individual to have a heighten } \\
\text { response to potential danger. }\end{array}$ & $\begin{array}{l}\text { (Castro \& Matt 1997; } \\
\text { Woodley et al. 2000) }\end{array}$ \\
\hline
\end{tabular}




\section{Endocrine plasticity}

What is endocrine plasticity? Endocrine plasticity is the reversible change in endocrine traits in response to unpredicted environmental changes (opposed to natural cyclical changes that occur within an individual), sometimes referred to as 'endocrine flexibility' (for a review see Taff \& Vitousek 2016). In this thesis, I largely focus on the glucocorticoid hormone cortisol (but also examine testosterone levels in Chapter 5). Cortisol is released from the adrenal cortex in response to activation of the hypothalamus-pituitary-adrenal (HPA) axis (mammals) or hypothalamus-pituitaryinterrenal (HPI) axis (fish; Barton 2002; Nelson 2011; Schreck \& Tort 2016).

When exposed to a stressor (a condition, agent or other physiological or psychological stimulus that causes stress to an individual) the nervous system is activated causing a cascade of chemical reactions, which helps an individual overcome the stressor and return to homeostasis (stable internal state Sapolsky et al. 2000; Nelson 2011). There are two neuroendocrine pathways; the sympathetic-adrenal-medullary axis and the hypothalamic-pituitary-adrenal/interrenal (HPA/I) axis (Barton 2002; Nelson 2011; Schreck \& Tort 2016). Corticotrophin-releasing-hormone is released from the hypothalamus, which in turn stimulates adrenocorticotropin-releasing hormone to be released from the pituitary gland which stimulates release of glucocorticoids (cortisol or corticosterone, depending upon the species) from the adrenal cortex (Nelson 2011; Wosu et al. 2013). The HPA/I axis response is rapid, and within minutes of activation circulating glucocorticoid concentrations increase, causing physiological and behavioural changes that help an individual to respond to the stressor (Schoenemann \& Bonier 2018). The HPA/I axis is mediated via a negative feedback system, when an individual is no longer exposed to a stressor or when blood glucocorticoid levels have reached a threshold to cope with the stressor the hypothalamus will no longer be activated and will return to homeostasis (Nelson 2011). This response of increasing glucocorticoids through activation of the HPA/I axis to deal with a stressor is known as the allostatic response, and coping with the stressor and returning to homeostasis is the overall function of the HPA/I axis (Madliger \& Love 2016; Schoenemann \& Bonier 2018).

Flexibility in glucocorticoid responses has been shown in a range of species and for a variety of physical environment changes, e.g. increase in glucocorticoids in response 
to novel environments (Hennessy et al. 2009; Colson et al. 2012; Miranda-de la Lama et al. 2012), low food availability (Blom et al. 2000; Wingfield \& Kitaysky 2002; Clinchy et al. 2004; Kitaysky et al. 2007; Behie et al. 2010; Herring et al. 2011; Chapman et al. 2015; Fardi et al. 2018), and handling of individuals (Pankhurst et al. 1992; Smith et al. 1994; Silverin et al. 1997; Grutter \& Pankhurst 2000; Kenagy \& Place 2000; Meka \& McCormick 2005; Cockrem et al. 2008; Adams et al. 2011). Furthermore, elevations in glucocorticoids have been demonstrated in socially isolated individuals (Smith \& French 1997; Dronjak et al. 2004; Ruscio et al. 2007; Banerjee \& Adkins-Regan 2011; Ferland \& Schrader 2011; Heimbürge et al. 2018) and in response to interactions with unfamiliar individuals (Castro \& Matt 1997; Woodley et al. 2000). Familiar individuals, on the other hand, can decrease glucocorticoids concentrations in social partners, via social buffering (Lyons et al. 1993; Bartolomucci et al. 2003; DeVries et al. 2003; Rukstalis \& French 2005; Hennessy et al. 2009). In addition, familiar partners can bidirectionally influence each other's physiological stress response ('cortisol coregulation' see Sbarra \& Hazan 2008 for a review; Saxbe \& Repetti 2010; Liu et al. 2013; Buttner et al. 2015; Saxbe et al. 2015; Fürtbauer \& Heistermann 2016; Cunningham 2017).

\section{Inter-individual differences in glucocorticoid concentrations}

Inter-individual differences in glucocorticoid concentrations reflect the different level of glucocorticoids produced by the HPA/I axis (Nelson 2011; Guindre-Parker 2020). It is thought that there should be individual differences in baseline glucocorticoids and plasticity responses (Guindre-Parker 2020), and there is increasing evidence for interindividual differences in glucocorticoids (see below). This is because the main role of baseline glucocorticoids is to maintain homeostasis, which is likely to be different for each individual as glucocorticoids are released at different rates from individuals' HPA/I axes (Guindre-Parker 2020). Plasticity in glucocorticoids occurs because individuals shift their energetic requirements to match the optimal phenotype for an environmental change (Guindre-Parker 2020). If individual differences in baseline glucocorticoids exist, we can also expect differences in plasticity because individual baseline glucocorticoids are closer or further away from the optimal phenotype (Guindre-Parker 2020). Inter-individual differences in endocrine traits are important to consider when studying endocrine plasticity because it will help us to understand 
how different individuals ('endocrine personalities'; see Fürtbauer et al. 2015b) react to environmental changes (Guindre-Parker 2020).

Individual variation in baseline glucocorticoids has been documented in various species (for a review see Guindre-Parker 2018), including chimpanzees (Pan troglodytes Sonnweber et al. 2018), tree swallows (Tachycineta bicolor Madliger \& Love 2016) and Fijian frogs (Platymantis vitiana Narayan et al. 2013). Additionally, individual variation in plasticity of glucocorticoid responses has also been shown in various species (for a review see Cockrem 2013), including chimpanzees (Anestis et al. 2006), spotted hyenas (Crocuta crouta Van Jaarsveld \& Skinner 1992), red squirrels (Sciurus vulgaris Guindre-Parker et al. 2019), hamsters (Phodopus campbelli Guimont \& Wynne-Edwards 2006), Adélie penguins (Pygoscelis adeliae Cockrem et al. 2006, 2009; Cockrem 2013), grey-faced petrel chicks (Pterodroma macroptera gouldi Adams et al. 2005), blue tits (Parus caeruleus Müller et al. 2006), hens (Gallus gallus domesticus Littin \& Cockrem 2001), Fijian frogs (Narayan et al. 2010, 2012a), cane toads (Rhinella marina Narayan et al. 2012b), African catfish (Clarias gariepinus Martins et al. 2006) and flatfish (Solea senegalx Silva et al. 2010).

There are several different hypotheses that have been proposed to explain the function of this inter-individual variation in endocrine traits, broadly split into fitness-related and evolutionary-related hypotheses. Guindre-Parker (2018) reviewed three fitness related hypotheses to explain inter-individual variation in baseline glucocorticoids. First the "Cort-Fitness hypothesis" assumes a negative relationship between cortisol and fitness, i.e. individuals with higher baseline cortisol will have lower fitness because this triggers allocation of resources away from coping with the environment, thus reducing fitness (shown in various species; Romero \& Wikelski 2001; Eeva et al. 2003; Altmann et al. 2004; Pride 2005; Angelier et al. 2007; Blas et al. 2007; Buck et al. 2007; Ellenberg et al. 2007; Müller et al. 2007; see Bonier et al. 2009 for a review; Schoenemann \& Bonier 2018; Guindre-Parker 2018). Second, the "Cort-Trade Off hypothesis" assumes that elevated cortisol increases reproductive success but decreases survival (Wingfield \& Sapolsky 2003; Schoenle et al. 2018). Third, the "Cort-Adaptation hypothesis" assumes that elevations in cortisol prepare individuals for future energetic demands (e.g. breeding, sessional weather changes, or challenge by a conspecific Guindre-Parker 2018; Bonier \& Cox 2020). 
With regards to the evolutionary hypotheses, two main hypotheses have been proposed to explain plasticity in endocrine responses. First, the "optimal endocrine hypothesis", where individual variation in plastic glucocorticoid responses are generated through responses to environmental change (Bonier \& Cox 2020). This hypothesis assumes that inter-individual variation in glucocorticoid plasticity responses enables expression of near-optimal endocrine phenotypes to overcome environmental change (Bonier \& Cox 2020). Variation in phenotypic expression exists because optimal endocrine response is going to be different for different environments and therefore it would be advantageous for individuals to be able to respond differently in different environments (Cockrem 2013b; Miles et al. 2018). For example, releasing a larger amount of cortisol in an environment where a predator is present (compared to one without a predator) - this will heighten the physiological response and shift energy from storage to energy use and help individuals cope with predator presence (Nelson 2011; Miles et al. 2018).

Second, the "ongoing selection hypothesis", assumes variation is naturally expressed within individuals, and that some individuals express suboptimal phenotypes which are simply selected against during environmental change (Bonier \& Cox 2020). Using the same predator versus non-predator example as above, under the "ongoing selection hypothesis", individuals with lower baseline cortisol would simply be selected against and would not survive. On a population level, inter-individual variation in baseline cortisol and cortisol plasticity is advantageous for populations surviving environmental changes (Miles et al. 2018). If a population included individuals with different baseline and cortisol responses to changing conditions, then the chances of some individuals being able to respond to the change and surviving are higher compared to a population consisting of individuals with identical endocrine responses (Miles et al. 2018; Sonnweber et al. 2018).

Whilst inter-individual variation in both baseline glucocorticoids and plasticity of glucocorticoid responses have been demonstrated (e.g. Adams et al. 2005; Martins et al. 2006; Silva et al. 2010; Cockrem 2013b; Narayan et al. 2013; Madliger \& Love 2016; Guindre-Parker 2018; Sonnweber et al. 2018), it remains largely unknown whether baseline glucocorticoid variation can predict plasticity, i.e. do inter-individual baseline differences predict inter-individual differences in glucocorticoid plasticity in response to a novel environment. Addressing this will help researchers to better 
understand how individuals differ in their ability to adapt to environmental changes and whether any observed variation in glucocorticoid plasticity can shape individual fitness (Dingemanse \& Wolf 2013; Cockrem et al. 2017; Guindre-Parker 2020).

\section{Linking endocrine measures to behaviour}

Hormones influence behaviour and vice versa, and it is therefore assumed that endocrine (cortisol) and behavioural responses should be correlated (Nelson 2011). Inter-individual differences in behavioural responses have been documented (both baseline behavioural responses and changes in behaviour in response to changing environment conditions; Hau \& Goymann 2015; Cockrem et al. 2017; Figure 2.1), and therefore it is assumed there would be inter-individual differences in related cortisol and behaviour responses.

Coping styles are coherent sets of consistent physiological and behavioural traits, and two distinct coping styles have been recognised; proactive and reactive individuals (for a review see Koolhaas et al. 1999). Proactive individuals are characterised by low HPA axis activity, high physical activity, aggression and are behaviourally less flexible (reviewed by Koolhaas et al. 1999; Coppens et al. 2010). Reactive individuals, in contrast, are characterised by high HPA axis activity, immobility, avoidance behaviour and are behaviourally more flexible (reviewed by Koolhaas et al. 1999, 2010; Coppens et al. 2010). An explanation for why this relationship exists could be that in reactive individuals, the higher production of cortisol triggers an increase in metabolism and reallocation of energy (Nelson 2011). This reallocation of energy can promote immobility and avoidance by making less energy available for movement (Nelson 2011).

Coping styles have been documented in many vertebrate species including Barbary macaques (Macaca sylvanus Tkaczynski et al. 2019), short-tailed singing mouse (Scotinomys teguina Crino et al. 2010), great tits (Parus major Carere \& Van Oers 2004; Baugh et al. 2012), rock agama (Psammophilus dorsis Batabyal \& Thaker 2019), zebrafish (Danio rerio Tudorache et al. 2013, 2015), rainbow trout (Oncorhynchus mykiss Ruiz-Gomez et al. 2008, 2011), brown trout (Salmo trutta Brelin et al. 2005), European seabass (Dicentrarchus labrax Ferrari et al. 2015), Nile tilapia (Oreochromis niloticus Martins et al. 2011) and three-spined stickleback fish (Gasterosteus aculeatus Bensky et al. 2017). However, it remains unknown whether 
coping styles are consistent across contexts; are the individual coping styles expressed under baseline conditions the same as coping styles expressed after an environmental change.

\section{Thesis objectives and data chapters}

In this thesis, I aim to gain a more in-depth understanding of variation in both shortand long-term endocrine plasticity over a range of physical and social environmental changes across different vertebrate systems, and the potential behavioural/morphological correlates. Phenotypic plasticity is important for individuals to cope with environmental changes (Windig et al. 2004; Gianoli \& Valladares 2012), and endocrine plasticity has been shown in response to physical and social environmental changes (Castro \& Matt 1997; Colson et al. 2012; Galhardo et al. 2012; Miranda-de la Lama et al. 2012). However, focusing on between-individual variation in endocrine plasticity will help researchers to better understand why and how plasticity occurs. I address five specific research questions around individual differences in endocrine responses, using three different vertebrate species (fish, humans, and sheep) and a variety of non-invasive methods for hormone analysis (water, saliva, and wool; Table 1.2; see Chapter 2 for an overview of methods and study systems). An outline of each data chapter is presented below. 
Table 1.2: Summary of data chapter (3-6), research questions (ticks indicate whether a question is addressed in a data chapter), and sample type for hormone analysis.

\begin{tabular}{|c|c|c|c|c|}
\hline Research question (Q) & $\begin{array}{l}\text { Chapter } 3 \\
\text { (three-spined } \\
\text { sticklebacks) }\end{array}$ & $\begin{array}{l}\text { Chapter } 4 \\
\text { (three-spined } \\
\text { sticklebacks) }\end{array}$ & $\begin{array}{c}\text { Chapter } 5 \\
\text { (humans) }\end{array}$ & $\begin{array}{c}\text { Chapter } 6 \\
\text { (sheep) }\end{array}$ \\
\hline $\begin{array}{l}\text { Q1. Are individuals } \\
\text { repeatable in their } \\
\text { endocrine traits? }\end{array}$ & $\checkmark$ & & $\checkmark$ & $\sqrt{ }$ \\
\hline $\begin{array}{l}\text { Q2. Do individuals show } \\
\text { plasticity in endocrine } \\
\text { traits ('endocrine } \\
\text { plasticity') and are they } \\
\text { repeatable therein? }\end{array}$ & $\checkmark$ & & $\sqrt{ }^{*}$ & $\checkmark$ \\
\hline $\begin{array}{l}\text { Q3. Does between- } \\
\text { individual variation in } \\
\text { endocrine traits predict } \\
\text { plasticity therein? }\end{array}$ & $\sqrt{ }$ & & $\checkmark$ & $\checkmark$ \\
\hline $\begin{array}{l}\text { Q4. Does between- } \\
\text { individual variation in } \\
\text { endocrine traits relate to } \\
\text { behaviour/morphological } \\
\text { condition? }\end{array}$ & & $\begin{array}{c}\checkmark \\
\text { (behaviour) }\end{array}$ & $\begin{array}{c}\checkmark \\
\text { (behaviour) }\end{array}$ & 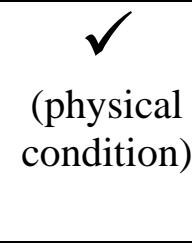 \\
\hline $\begin{array}{l}\text { Q5. Does the endocrine } \\
\text { status of social partners } \\
\text { affect endocrine } \\
\text { plasticity? }\end{array}$ & $\checkmark$ & & & \\
\hline Sample type & \multicolumn{2}{|c|}{ Waterborne } & Saliva & Wool \\
\hline
\end{tabular}

*Endocrine plasticity measured, but due to experimental set up repeatability in plasticity was not assessed in this chapter

In Chapter 3, I investigate the effects of recent social context on cortisol responses in three-spined stickleback fish (Gasterosteus aculeatus). I placed dyads of fish twice in a novel environment and collected waterborne hormone samples before and after. This allowed me to investigate cortisol repeatability (Q1) and plasticity (Q2) and to test whether between-individual variation in HPI axis activity predicted endocrine plasticity (Q3). Manipulating familiarity status (familiar versus unfamiliar) and recent housing conditions (socially versus solitarily housed), allowed me to investigate effects of social context on partners' synchrony in cortisol responses (coregulation; Q5). I also investigate whether partner or focal baseline cortisol concentrations predict changes in cortisol levels in responses to a novel environment (i.e. level of plasticity; Q5). 
In Chapter 4, I investigate effects of familiarity/recent social context on short-term repeatability and behavioural responses in three-spined stickleback fish. Moreover, I combine behavioural measures with hormonal data (Chapter 3 ) to investigate potential links between behavioural and hormonal responses (i.e. coping styles; Q4).

In Chapter 5, I investigate endocrine plasticity in humans (Homo sapiens) and its potential links to BIG 5 personality measures as well as perceived stress (using questionnaires) in a social task. First, I test whether endocrine response (cortisol and testosterone) and perceived stress are repeatable (Q1) and assess potential changes in hormone concentrations and perceived stress in response to the social task (Q2). I also assess whether between-individual variation in endocrine traits predicts plasticity therein (Q3). Second, I investigate whether individual cortisol concentrations are related to perceived stress and/or personality traits (Q4).

In Chapter 6, I investigate long-term endocrine plasticity in Welsh mountain ewes (Ovis aries) in response to changing food availability. First, I confirm changes in body mass (weight gain) following two periods of grazing in a high-quality upland pasture. Second, I investigate repeatability and plasticity of wool cortisol concentration (WCC) during these periods (Q1 and Q2), and test whether between-individual variation in WCC predicts plasticity (Q3). Finally, I investigate whether changes in WCC are linked to changes in body mass (Q4). 


\section{Chapter 2: Methods and study systems}

In this thesis I use a variety of non-invasive endocrinological techniques as well as a range of vertebrate study systems to measure endocrine traits to tackle my research questions (see Table 1.2; Chapter 1). In this chapter, I briefly outline the methods used and review my study systems.

\section{Methods}

Glucocorticoids can be quantified non-invasively from a variety of sample types, including saliva, urine, faeces, water and hair (reviewed by Scott \& Ellis 2007; Behringer \& Deschner 2017; Burnard et al. 2017). Non-invasive collection does not induce stress associated with blood collection, which is important as the samples will represent HPA/I axis response to environment change only, rather than being influenced by the collection process (Levine et al. 2007; Gatti et al. 2009). There are different lag times between hormone secretion and appearance of each non-invasive sample type and the choice of which to use will depend on the research question and the feasibility of sample collection (Behringer \& Deschner 2017). If the research question is investigating short-term hormone production and changes, sample types in which cortisol is secreted within minutes are needed (Behringer \& Deschner 2017). Saliva and water hormone secretion can be detected within minutes (reviewed by Lewis 2006; Scott \& Ellis 2007; Figure 2.1). If the research question is investigating long-term hormone production and changes, sample types in which cortisol is secreted over weeks or months are needed to capture the long-term measure of cortisol (reviewed by Behringer \& Deschner 2017; Burnard et al. 2017; Figure 2.1). In the present thesis, I quantify hormone concentrations from saliva (humans; Chapter 5), water (fish; Chapters 3 and 4), and wool samples (sheep; Chapter 6). I discuss the basic principles of each method below. 


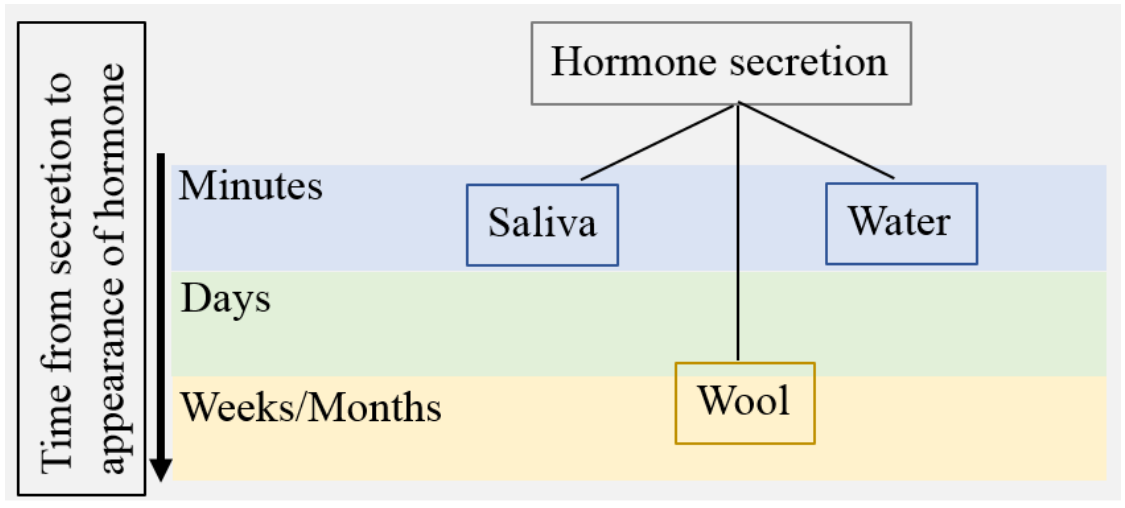

Figure 2.1: Overview of the time from hormone secretion to appearance in noninvasive sample types (adapted from Behringer \& Deschner 2017).

\section{Hormones from water}

In some aquatic animals, such as fish, steroids are released from the gills, via passive diffusion due to the concentration gradient between plasma and the water, therefore cortisol can be extracted from water samples and a number of studies have demonstrated a correlation between waterborne and plasma cortisol (Vermeirssen \& Scott 1996; Ellis et al. 2005; reviewed by Scott \& Ellis 2007). To obtain a water sample, an individual is placed into a beaker filled with a set amount of water (dependent on the species the sample is collected from) and left for a set amount of time (e.g. $1 \mathrm{hr}$; during this time the hormones are released, via passive diffusion, from the gills (reviewed by Scott \& Ellis 2007). Water samples are filtered, to remove any faecal matter, and stored frozen until analysis (see Scott et al. 2008 for a review; Baugh et al. 2018). Solid phase extraction methods are used to extract steroids from the water sample, and standard enzyme immunoassays kits can be used to measure steroid hormone concentrations (Figure 2.2; see Chapter 3 for full methods).

This method has many advantages for small aquatic individuals; it reduces the stress of handling associated with blood samples and steroids found in water are integrated through time (i.e. via diffusion), which means it can represent hormone levels over time (reviewed by Scott \& Ellis 2007). Most importantly, extracting hormones from water samples allows for repeated measures (not possible with plasma samples due to the stickleback's small size; Scott \& Ellis 2007), which are crucial for any study investigating repeatability and plasticity in endocrine traits. Waterborne cortisol has been successfully measured across a range of aquatic species, including common carp 
(Cyprinus carpio Ruane \& Komen 2003), rainbow trout (Oncorhynchus mykiss Ellis et al. 2004), guppies (Poecilia reticulata Fischer et al. 2014), poecilid fish (Brachyrhaphis episcopi Archard et al. 2012), zebrafish (Danio Rerio Pavlidis et al. 2013) and three-spined stickleback fish (Gasterosteus aculeatus Sebire et al. 2007; Fürtbauer et al. 2015; Fürtbauer \& Heistermann 2016). In Chapter 3, I use water samples to investigate the effects of recent social context on cortisol responses in threespined stickleback fish.

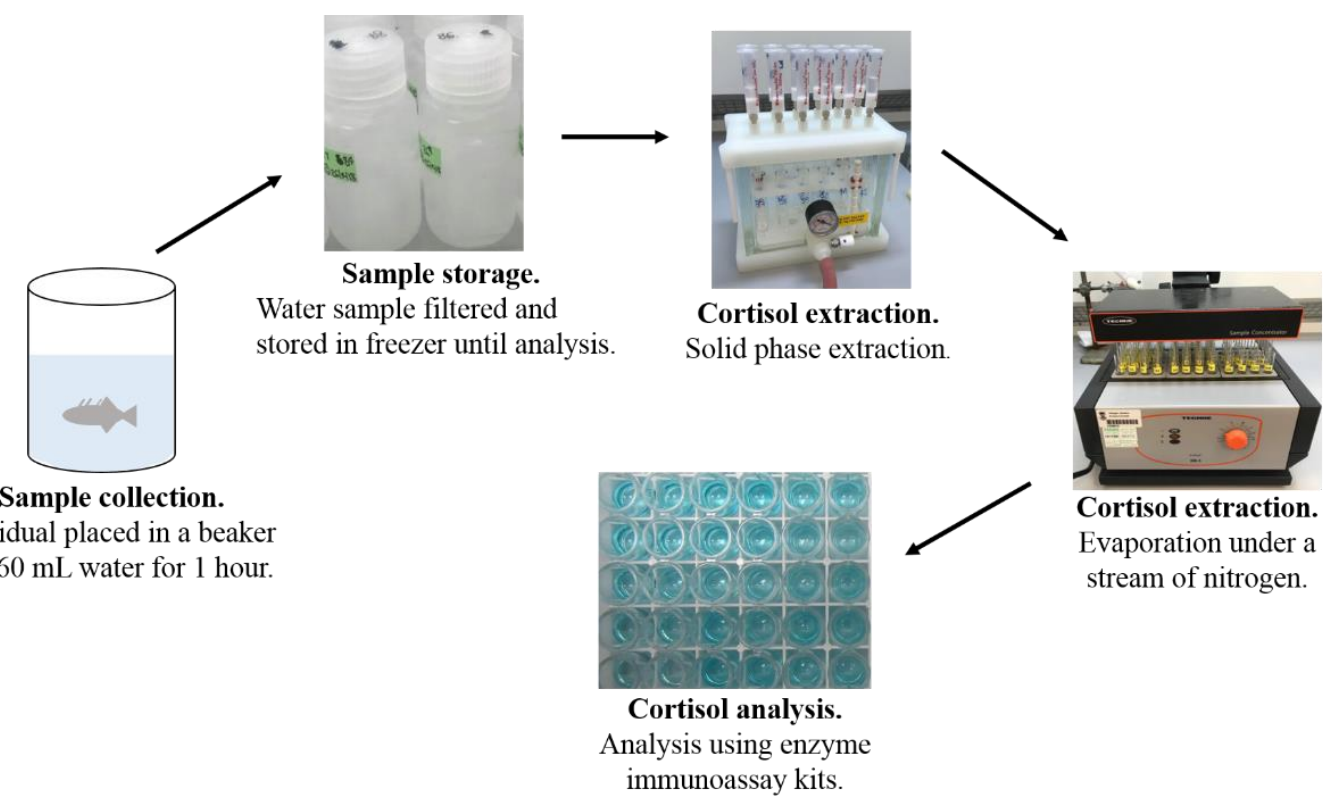

Figure 2.2: Schematic diagram of waterborne hormone sampling, extraction and analysis methodology.

\section{Hormones from saliva}

Hormones found in saliva reflect the unbound plasma hormone concentrations (Gröschl 2009). Many hormones can be found in saliva, including steroid hormones such as testosterone and cortisol (Gröschl 2009). Saliva samples represent real-time cortisol concentrations and can represent short-term changes in cortisol concentrations (Teruhisa et al. 1981; Behringer \& Deschner 2017; see Figure 2.1). High correlations have been shown between saliva and serum cortisol (Lewis 2006; Hellhammer et al. 2009; Kobayashi \& Miyazaki 2015) and testosterone (Dabbs 1990; Lippi et al. 2016; Francavilla et al. 2018) in validation studies. In humans, saliva samples are collected from individuals depositing their own saliva into a tube using a saliva collection aid; 
only a small amount (approximately $1 \mathrm{~mL}$ ) is needed (Hofman 2001). Once samples are collected, they are stored frozen until analysis, using enzyme immunoassays (see Chapter 5 for full details; Hofman 2001). The advantages of saliva collection for hormone analysis in humans is that it can be self-administered so a medical professional or special equipment is not needed (Adam \& Kumari 2009).

Salivary hormone extraction and analysis has been applied across several species including humans (Homo sapiens Thomas et al. 2009; Pluess et al. 2010; Hellhammer \& Schubert 2012; Matsuda et al. 2012; Stawski et al. 2013; Rudolph et al. 2016), chimpanzees (Pan troglodytes Heintz et al. 2011), common marmosets (Callithrix jacchus Ash et al. 2018), rhesus macaques (Macaca mulatta Pfefferle et al. 2018), dairy cows (Bos taurus Dzviti et al. 2019; Riek et al. 2019) and domestic dogs (Canis lupus familiaris Bergamasco et al. 2010; Glenk et al. 2014). In Chapter 5, I use saliva samples to measure cortisol and testosterone concentrations in human subjects in order to investigate endocrine plasticity and its potential links to BIG 5 personality measures and perceived stress in a social task.

\section{Hormones from hair/wool}

Cortisol measurements from hair offer a non-invasive approach for the measurement of long-term HPA axis activity (Raul et al. 2004; Meyer \& Novak 2012; Russell et al. 2012; Stalder \& Kirschbaum 2012; Burnard et al. 2017). Whilst the exact method of incorporation of cortisol into hair is not known, the route of incorporation is thought to be from the vascular supply, into the follicular cells that make up the hair shaft (Meyer \& Novak 2012; Russell et al. 2012). This incorporation occurs over time, which is why hair samples represent long-term HPA axis activity (Meyer \& Novak 2012; Russell et al. 2012).

Despite hair growth rates varying across species, several studies suggest that hair cortisol measures can be used as a retrospective calendar of HPA axis activity (Raul et al. 2004; Kirschbaum et al. 2009; Meyer \& Novak 2012; Carlitz et al. 2014; Burnard et al. 2017; Fürtbauer et al. 2019). Hair sample collection is relatively simple, depending on the species. In humans a sample can be easily cut from the back of the head (D'Anna-Hernandez et al. 2011; van Holland et al. 2012), in cattle and domestic species that are regularly/easy to handle, samples can be taken through simple restrain (Comin et al. 2011; Roth et al. 2016; Grigg et al. 2017; Peric et al. 2017; Fürtbauer et 
al. 2019), whereas wild primate species would usually need to be placed under anaesthetic (Fairbanks et al. 2011; Grant et al. 2017). Samples can be stored at room temperature for extended periods before extraction and analysis, providing a major advantage (Raul et al. 2004; D'Anna-Hernandez et al. 2011). A hair sample is taken by shaving as close to the skin as possible, with the proximal end of the hair shaft being used for analysis (Sauvé et al. 2007; Meyer \& Novak 2012). The hair sample is cut to represent the period of growth of interest, whilst this is species dependent, usually around $1 \mathrm{~cm}$ of hair represents growth in the last month (Raul et al. 2004; Russell et al. 2012). Upon collection, hair samples are stored in aluminium foil at room temperature until extraction and analysis (Davenport et al. 2006; Ghassemi Nejad et al. 2014; Stubsjøen et al. 2015; Caslini et al. 2016). To extract steroid hormones, samples are first washed and dried, then cut, weighed and finally methanol is added for incubation (see Chapter 6 for full details). Following a 24-hour extraction period, steroid-containing supernatant is transferred to a glass tube which is completely dried under a stream of nitrogen gas (Raul et al. 2004; Meyer \& Novak 2012; Burnard et al. 2017). Once dried, enzyme immunoassays are used to quantify hormone concentrations (see Figure 2.3 for a schematic methodology diagram).

Hair cortisol measurements have become increasingly popular and have been used across numerous species including cows (Bos taurus Comin et al. 2011; Moya et al. 2013; Peric et al. 2017), domestic pigs (Sus scrofa domesticus Casal et al. 2017), red deer (Cervus elaphus Caslini et al. 2016), domestic cats (Accorsi et al. 2008) and dogs (Canis lupus familiaris Accorsi et al. 2008; Bennett \& Hayssen 2010; Siniscalchi et al. 2013; Roth et al. 2016; Grigg et al. 2017), rhesus macaques (Macaca mulatta Davenport et al. 2006, 2008; Hamel et al. 2017), pig-tailed macaques (Macaca nemestrina Grant et al. 2017), vervet monkeys (Chlorocebus pygerythrus Fairbanks et al. 2011) and humans (D'Anna-Hernandez et al. 2011; van Holland et al. 2012).

Due to the increasing interest in agricultural welfare, wool cortisol concentrations have also been used to asses long-term HPA axis activity in sheep (Ghassemi Nejad et al. 2014; Stubsjøen et al. 2015; Salaberger et al. 2016; Nejad et al. 2017; Fürtbauer et al. 2019). Whilst, wool fibres have different characteristics from hair, the continuous growth of the wool fibres mean that they can be used as a retrospective calendar of HPA axis activity (Ghassemi Nejad et al. 2014; Burnard et al. 2017). Wool samples are collected in a similar way as hair, with wool being shaved as close to the skin as 
possible with the proximal end of the sample being used for analysis (Burnard et al. 2017; Fürtbauer et al. 2019). Extraction and analysis of wool samples mirror those described above for hair samples (see Chapter 6 for full methodology on wool cortisol extraction and analysis). In Chapter 6, I use wool for cortisol analysis in Welsh Mountain ewes in order to investigate long-term endocrine plasticity in response to changing food availability. Using a measurement of long-term HPA axis enabled me to match cortisol measurements over the last month, to the change in food availability over the same period.

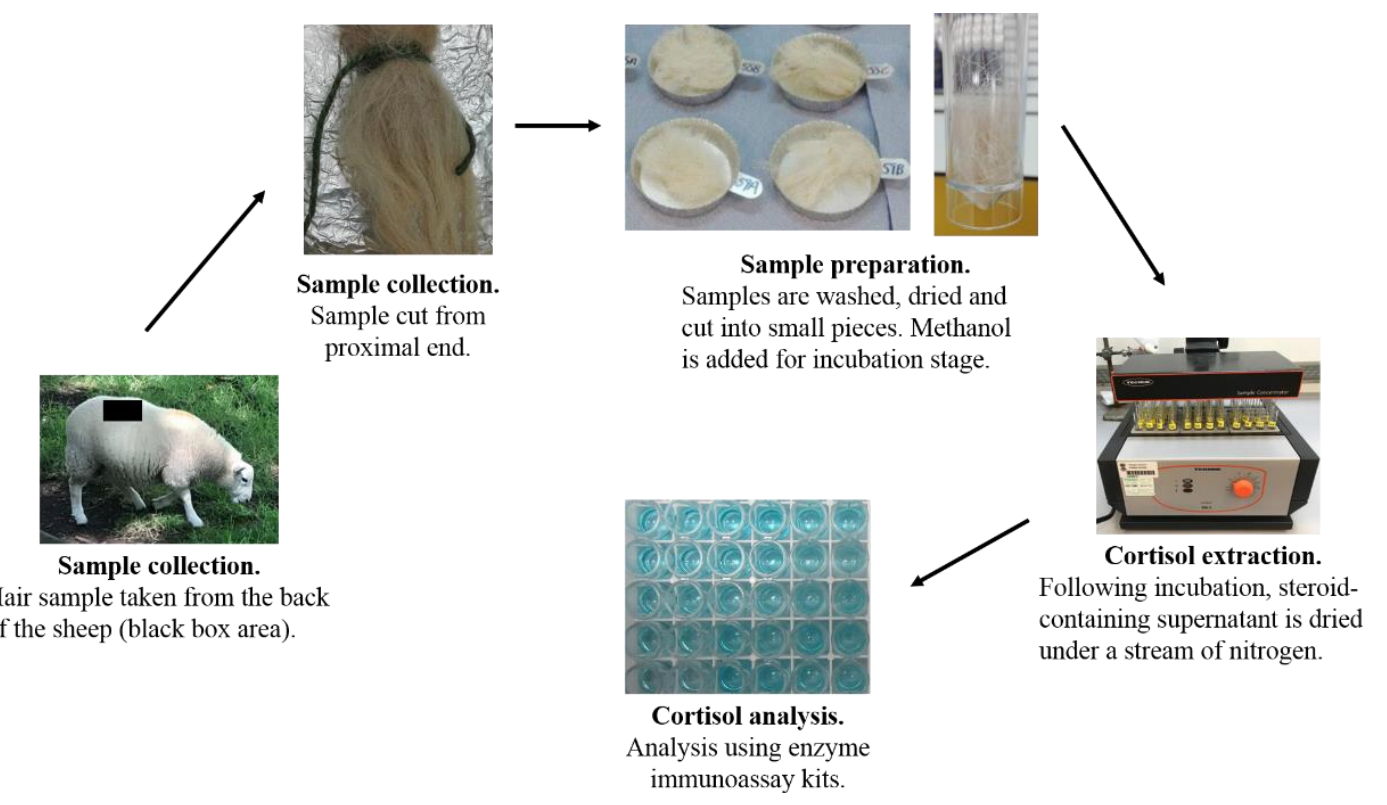

Figure 2.3: Schematic diagram for methodology of wool collection and cortisol extraction and analysis. 


\section{Study systems}

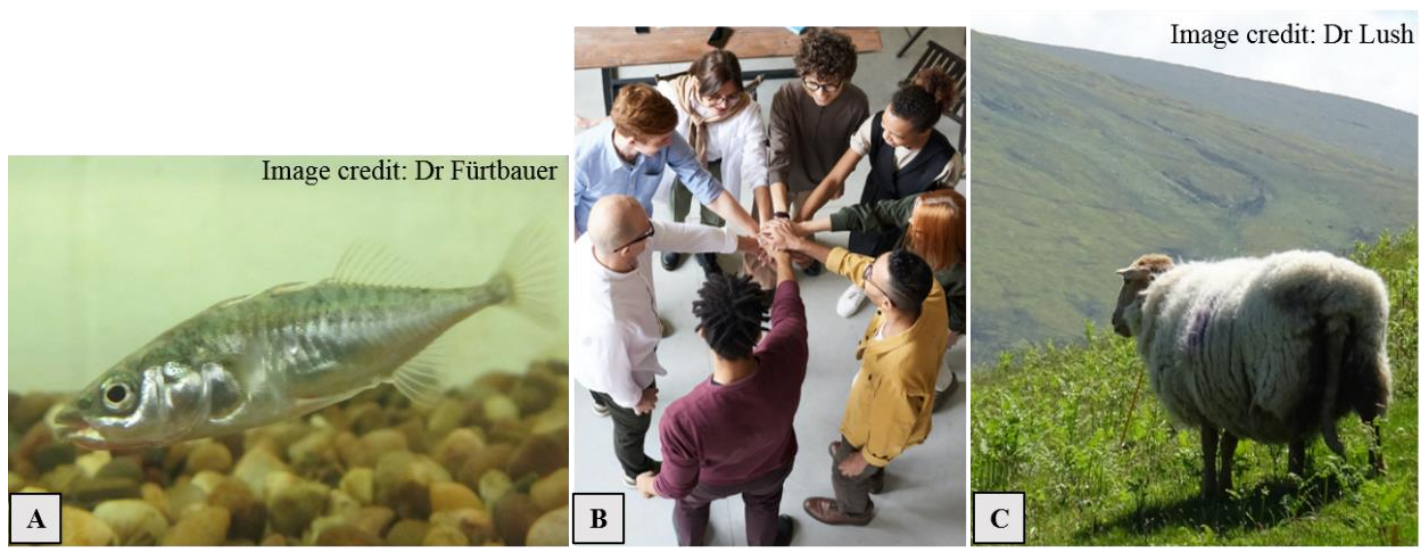

Figure 2.4: Study systems used in my thesis; A) Three-spined stickleback fish, B) Humans and C) Welsh mountain ewes.

\section{Stickleback fish}

Three-spined stickleback fish are excellent models to use to explore behavioural endocrinological research questions. They are naturally social species that usually shoal together, and therefore are good models to explore behavioural responses and social interactions (Huntingford \& Ruiz-Gomez 2009). They are also a relatively robust species, and are able to be housed either individually or in groups in a laboratory setting, which allows easy investigation into effects of recent housing conditions on individual behaviour (Huntingford \& Ruiz-Gomez 2009; Jolles et al. 2016). Previous studies have used three-spined stickleback fish to investigate group behaviour and the effects of familiarity (Ward et al. 2004; Ward \& Hart 2005) and recent social conditions (Jolles et al. 2016). Additionally, in three-spined stickleback fish waterborne cortisol measurements have been used to investigate daily patterns of cortisol measurements (Sebire et al. 2007), predator effects (Fürtbauer et al. 2015b), cortisol coregulation (Fürtbauer \& Heistermann 2016), and coping styles (Bensky et al. 2017). Waterborne cortisol measurements have also been taken to investigate the effects of hypoxia (O'Connor et al. 2011), water treatment (Pottinger et al. 2011) and climate change related factors (Pottinger \& Feuchtmayr 2020) on HPI axis activity.

\section{Humans}

Humans as a model species for biological studies have many advantages (Brosnan \& Postma 2017; Sterelny 2017). There is a broad understanding of human processes and 
therefore research encompasses a range of areas including, genetics, behaviour, neurology, endocrinology, diseases, morphology and environmental interactions (Brosnan \& Postma 2017; Sterelny 2017). Additionally, as humans are the only species that can state their thoughts and feelings, they provide a large amount of data covering a range of topics (e.g. family and friend relationships, education and medical issues; Brosnan \& Postma 2017). A range of questionnaires have been developed to understand individuals' thoughts, feelings and interactions with others, including personality, relationship and daily challenges/stressors questionnaires as well as cognitive tasks (Cohen et al. 1983; Goldberg 1990; Kirschbaum et al. 1993).

Endocrine measures are also relatively easy to obtain from humans, as researchers can communicate with participates and explain processes, which helps with ease of sample collection (reviewed by Esteban \& Castaño 2009). As non-invasive endocrine measures provide researchers with the opportunity to assess HPA axis activity without inducing stress, non-invasive methods of collection in humans (i.e. saliva, hair and fingernail samples) have become increasingly popular over the last decade (reviewed by Esteban \& Castaño 2009). Saliva samples have been widely used to assess glucocorticoids in humans across a range of environments including working vs nonworking days (Marchand et al. 2013; Rudolph et al. 2016), night vs day shifts (Thomas et al. 2009), daily fluctuations (Dahlgren et al. 2009; Matsuda et al. 2012), effects of a social stress test (Hellhammer \& Schubert 2012; Allen et al. 2014), effects of daily stressors (Powell \& Schlotz 2012; Stawski et al. 2013), during pregnancy (Obel et al. 2005; Pluess et al. 2010) and for clinical research (Jogems-Kosterman et al. 2007; Restituto et al. 2008; Kudielka et al. 2009). Additionally, saliva samples have been used to measure cortisol and testosterone to investigate their role in friendship formation (Kornienko et al. 2014, 2016; Ketay et al. 2017), during competitive sports (Edwards \& Kurlander 2010; Edwards \& Casto 2013; Casto et al. 2014; Casto \& Edwards 2016), social interactions (Eisenegger et al. 2011) and links to personality traits (Kirschbaum et al. 1992; Shoal et al. 2003; Parent-Lamarche \& Marchand 2015; Smeets-Janssen et al. 2015; Afrisham et al. 2016).

Sheep

Sheep are a domesticated species, living in a mixture of indoor and outdoor environments, which provides researchers with an opportunity to explore biological 
processes across different environments (Pinnapureddy et al. 2015; Burnard et al. 2017). Sheep have been widely used for medical and genetic studies due to their longer lifespan enabling the ability to conduct chronic studies and similarity of genetic and physiological composition to humans (Jennifer Morton \& Howland 2013; Pinnapureddy et al. 2015). It has been shown that sheep possess a good cognitive ability, have good facial recognition and are able to perform decision-making tasks making them a good model for behavioural studies (Kendrick et al. 2001; Reefmann et al. 2009; Doyle et al. 2011; Morton \& Avanzo 2011). Additionally, as a domesticated species, they are frequently handled which provides opportunities for measures to be routinely taken, an advantage for longitudinal research (Pinnapureddy et al. 2015).

Within the last decade, wool samples have been used to assess long-term HPA axis activity in sheep in response to heat stress and water restrictions (Ghassemi Nejad et al. 2014; Nejad et al. 2017), to assess external factors influencing cortisol concentrations (Salaberger et al. 2016), in response to induced stress (Weaver et al. 2020) and during breeding (Sawyer et al. 2019). Whilst, not currently explored in sheep, research has used hair cortisol concentrations to assess cortisol during movement between different grazing periods in cows (Comin et al. 2011; Peric et al. 2017). 


\title{
Chapter 3: Effects of recent social context and familiarity on cortisol responses in three-spined stickleback fish (Gasterosteus aculeatus)
}

\begin{abstract}
Social interactions can influence individuals' hormonal responses and where individuals experience a potentially stressful event together, there is potential for bidirectional influence of individuals' physiological stress response (i.e. 'coregulation'). Coregulation has been shown to occur in humans and fish, and especially between familiar (or related) individuals. This study investigates effects of recent housing conditions (socially versus solitarily housed) and familiarity on cortisol responses and potential cortisol coregulation in dyads of three-spined stickleback fish (Gasterosteus aculeatus) experiencing a novel environment (NE). Individual cortisol concentrations were repeatable but did not differ significantly between pre- and postnovel environment, indicating the novel environment was not perceived as a stressor. Neither pre- nor post-NE cortisol concentrations were affected by recent social context or familiarity in this study. I found no evidence for cortisol coregulation between pairs of fish either pre- or post- novel environment. Cortisol coregulation is usually stronger under acute stress, but here the novel environment was not perceived as stressful potentially explaining the lack of coregulation and supporting the idea that coregulation provides a mechanism for individuals to align their responses to challenging environmental changes. I found that individual but not partner cortisol concentration predicted plasticity in cortisol responses (i.e. the change in cortisol from pre- to post-test measures), suggesting that individual baseline measures are important indicators of HPI axis flexibility. In order to better understand the effects of recent social context and familiarity on cortisol responses, future research should investigate dyadic cortisol responses across a range of environments (stressful and non-stressful) to better understand what physical and social conditions affect cortisol responses.
\end{abstract}




\section{Introduction}

Social interactions occur frequently within groups of animals and can influence individual physiology, e.g. hormonal responses, and behaviour (DeVries et al. 2003). When exposed to a new, potentially risky environment, an individual's hypothalamicpituitary-adrenal (HPA) axis (mammals), or hypothalamic-pituitary-interrenal (HPI) axis (fishes) is activated (Barton 2002; Nelson 2011; Schreck \& Tort 2016). Activation of the HPA/HPI axis causes a cascade of events, which result in glucocorticoids (e.g. cortisol) being released from the adrenal cortex; this represents the physiological stress response (Nelson 2011). Whilst research has shown that an interaction with an unfamiliar individual can heighten this stress response (Castro \& Matt 1997; Woodley et al. 2000), it has also been shown that interactions between familiar individuals can reduce an individual's stress response and act as a buffer in stressful environments (reviewed by DeVries et al. 2003). An increasing amount of research has focused on exploring how the HPA/HPI axis responds to social interactions and how a focal individual's hormonal response (i.e. their level of endocrine plasticity) is influenced by a partner, particularly under stressful conditions (reviewed by DeVries et al. 2003).

Researchers have investigated how different social interactions and contexts affect individual glucocorticoid levels through investigating cortisol responses, including exposure to novel environments (Hennessy et al. 2009; Colson et al. 2012; Mirandade la Lama et al. 2012), removing and reintroducing a partner (Carter et al. 1995), and introducing unfamiliar individuals (Castro \& Matt 1997; Woodley et al. 2000). Based on these findings, two phenomena have emerged to explain the link between partner interactions and HPA/HPI axis activity: (1) social buffering and (2) cortisol coregulation (see DeVries et al. 2003; Sbarra \& Hazan 2008 for reviews; Saxbe \& Repetti 2010). Social buffering refers to the unidirectional process of another individual lowering a focal individual's cortisol concentration under stressful conditions (DeVries et al. 2007). Cortisol coregulation, in contrast, is the bidirectional influence of social partners; the regulation (up or down) of both dyad partners' physiological stress responses (see Sbarra \& Hazan 2008 for a review; Saxbe \& Repetti 2010; Fürtbauer \& Heistermann 2016).

Social buffering - shown to occur in species such as house mice (Mus musculus Bartolomucci et al. 2003; Hennessy et al. 2009), rats (Rattus spp. DeVries et al. 2003), 
guinea pigs (Cavia porecllus Hennessy et al. 2006), black tufted-ear marmosets (Callithrix kuhli DeVries et al. 2003; Rukstalis \& French 2005), squirrel monkeys (Saimiri sciureus DeVries et al. 2003) and sheep (Ovis aries Lyons et al. 1993; Hennessy et al. 2009) - highlights the importance of a partner's presence on a focal individual's cortisol response, but it does not allow for the investigation of both partners and how they may co-regulate each other's physiological stress responses in different environments. Cortisol coregulation, in contrast, takes into account both partner's physiological state and allows exploration into how they may co-regulate (see Sbarra \& Hazan 2008 for a review on human coregulation). In humans, cortisol coregulation has been shown to occur in married couples (Saxbe \& Repetti 2010; Papp et al. 2013), between mothers and infants (Neu et al. 2008; Mörelius et al. 2015) and between mothers and adolescent offspring (Papp et al. 2009). It is suggested that cortisol coregulation functions to reduce or inhibit the stress response of a social partner under conditions that they would normally perceive as stressful (Sbarra \& Hazan 2008; Saxbe \& Repetti 2010). Whilst the majority of coregulation work has been conducted on humans, recent work has started to explore this phenomenon in other species, i.e. fish dyads (Fürtbauer \& Heistermann 2016) and human-dog/dogdog dyads (Buttner et al. 2015; Cunningham 2017; Solman \& Fürtbauer in prep).

The recent work on coregulation in humans (Neu et al. 2008; Papp et al. 2009, 2013; Saxbe \& Repetti 2010; Liu et al. 2013; Mörelius et al. 2015; Saxbe et al. 2015) suggests that a close relationship is needed for coregulation to occur. However, currently it is not yet clear what level of partner familiarity is needed for coregulation to occur, and whether short-term familiarity can result in coregulation. Previous research into social buffering in black tufted-ear marmosets (Callithrix kuhlii Rukstalis \& French 2005), zebra finches (Taeniopygia guttata Remage-Healey et al. 2003) and guinea pigs (Cavia porcellus Hennessy et al. 2006, 2009) shows that a familiar housing partner is needed for buffering effects to occur. Whether this is also the case for cortisol coregulation is not known given the focus of correlation studies on married or cohabitating human dyads (Ditzen et al. 2007; Saxbe \& Repetti 2010; Liu et al. 2013; Papp et al. 2013) or between mothers and children (Neu et al. 2008; Papp et al. 2009; Mörelius et al. 2015).

Here, I investigated whether familiarity or recent social context affects cortisol responses/coregulation in dyads of three-spined sticklebacks (Gasterosteus aculeatus), 
using a novel environment test and a repeated-measures design to collect waterborne hormone samples. Previous research has shown when familiar stickleback dyads are introduced to an open field environment (a mild stressor) the dyad partners' cortisol concentrations are significantly positively correlated, indicating coregulation of HPI axis activity (Fürtbauer \& Heistermann 2016). Furthermore, baseline cortisol levels of dyad partners predicted focal fishes' cortisol responses, with individuals showing higher cortisol concentrations following the open field exposure if their partner had higher baseline cortisol concentrations (Fürtbauer \& Heistermann 2016), indicating that a familiar individual's hormonal state can influence partner responses. Whilst these results, for the first time, highlight that cortisol coregulation occurs in stressful situations in a non-human animal, it remains unknown if and how familiarity (comparing familiar vs unfamiliar dyads) or recent social context (socially versus solitarily housed) affect coregulatory processes.

This experiment was set up to manipulate recent social context and familiarity. Prior to the experiments, individuals were housed either socially (with another individual) or solitarily, and during the experiment's individuals were either paired with a familiar or unfamiliar conspecific. Firstly, I tested whether the novel environment was perceived as a stressor. Based on previous research, I expected the fish to show repeatability in their cortisol concentrations (Fürtbauer et al. 2015b; Prediction 1), and show an increase in cortisol in response to open field environments (Fürtbauer \& Heistermann 2016; Prediction 2). Secondly, I tested whether coregulation occurred within dyads and whether this was influenced by recent housing condition and/or familiarity. If 'simple' social interaction is sufficient for coregulation to occur, then coregulation should occur after the novel environment test in all dyads regardless of recent social context or familiarity. However, if coregulation requires familiarity between individuals, then it should only occur for individuals that are familiar and have been socially housed together prior to the experiments. Based on previous research showing coregulation in familiar dyads (Fürtbauer \& Heistermann 2016), I predicted that familiar dyads' post-novel environment (NE) cortisol concentrations would be correlated; that is, individuals would coregulate (Prediction 3). I also tested pairs of fish that had been housed socially but were paired with unfamiliar partners to test if recent social contact, but not familiarity with a social partner, is sufficient for coregulation to occur (Prediction 4). The repeat testing in the novel environment 
allowed me to also test the possibility that short-term familiarity (i.e. familiarity gained in the first novel environment trial, hereafter referred to as ' $\mathrm{NE1}$ ', in the unfamiliar dyads) would cause coregulation in the second exposure to the novel environment (hereafter referred to as 'NE2'; Prediction 5). See Figure 3.1 for a summary of experiment and conditions. Thirdly, I investigate whether (a) partner and/or (b) focal baseline cortisol concentrations predicted any changes (i.e. level of plasticity) in cortisol from pre- to post-NE. Previous research on stickleback fish has shown that partner baseline cortisol levels were linked to focal fishes' cortisol levels after a shared open field test (i.e. individuals had higher stress following the open field in their partner had high baseline cortisol; Fürtbauer \& Heistermann 2016). Based on this research, I predicted that higher partner baseline cortisol concentrations would result in higher cortisol post-NE exposure in focal fish (Prediction 6). I also tested whether individual baseline cortisol predicted any change in cortisol in response to the novel environment (i.e. investigating whether individuals with higher or lower baseline concentrations are more reactive; showed a greater level of plasticity; Taff \& Vitousek 2016; Stedman et al. 2017; Prediction 7).

\section{Material and methods}

\section{Subjects and housing}

$\mathrm{N}=119$ stickleback fish, sourced from a wild commercially bred population through CarpCo, Kent, England (www.carpco.co.uk), were initially housed in two large holding tanks $(122 \times 38 \times 30 \mathrm{~cm})$ for one week prior to the start of experiments. $\mathrm{N}=76$ fish ( $\mathrm{n}=56$ females, $\mathrm{n}=20$ males) were used in the experiments. The experiments were conducted between April and May 2018. Throughout the study, temperature of the laboratory/water was kept between $18-20{ }^{\circ} \mathrm{C}$, a constant light cycle of 8L:16D was kept and fish were fed defrosted bloodworms (Chironomus spp.) daily. Five days prior to experiments, fish were moved to a $2.8 \mathrm{~L}$ aerated holding tank (referred to as 'housing tanks' in the study) which were lined with gravel and included a plastic plant. Fish were either housed in pairs or solitarily ( $n=24$ dyad tanks, $n=24$ solitary tanks). Dyads were a mixture of same-sex ( $\mathrm{n}=17$ female-female, $\mathrm{n}=1$ male-male) and mixed-sex $(n=18)$ dyads. Where possible male-male dyads were avoided due to the fact that potentially fatal aggressive territorial behaviour can occur between males (McKinnon 
\& McPhail 1996; Tinghitella et al. 2018). Individual tanks were set up one day before fish were moved from the holding tanks, and the water source was the same as the housing tank. Fish were in the individual tanks for a total of five days; after two days of being in the individual tanks a 50\% water change was undertaken and following the next two days a full water change was undertaken. Fish were tagged for individual identification purposes the day before the experiments using $5 \mathrm{~mm}$ diameter electrical tap disks (green or yellow) which were placed on the middle dorsal spine following previous studies (Webster \& Laland 2009; Laskowski \& Bell 2013; Jolles et al. 2017). The tags were removed after the experiments. All procedures used in this study were approved by Swansea University's Ethics Committee (IP-1213-3).

\section{Experimental procedures}

All fish were tested in dyads and were subject to either social or solitary conditions in housing tanks and had a familiar or unfamiliar partner during the experiments. Therefore, there were three overall conditions: A) individuals housed solitarily and tested with an unfamiliar partner; $\mathrm{n}=12$ dyads, B) individuals housed socially but tested with an unfamiliar partner; $n=12$ dyads and C) individuals housed socially and tested with their familiar partner; $n=12$ dyads (Figure 3.1). For each dyad the experiment lasted 4 hours in total and included two exposures to a novel environment. The novel environment test tanks were identical to the tanks used by Fürtbauer \& Heistermann (2016) but included two shelters (ceramic flowerpots) on each end and were lined with white sand and filled up to $4 \mathrm{~cm}$ with water $(56 \times 23 \times 16 \mathrm{~cm})$. Different test tanks were used for NE1 and NE2 to rule out any potential olfactory cues because previous research has shown sticklebacks use chemical olfactory cues to recognise familiar settings (Ward et al. 2004). Water was added to test tanks the day before the experiment from the same source as the holding/individual tanks and kept aerated until the start of the experiment. Dyads were exposed to each NE test for 30 minutes (Figure 3.2). 


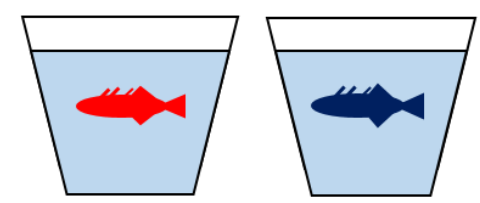

A

Solitary housed

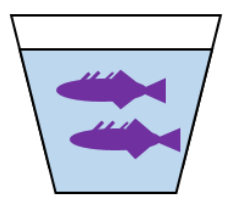

B

Pre-test sample

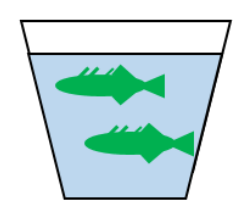

Socially housed

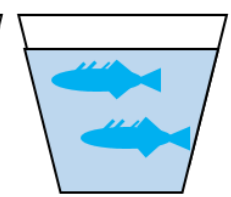

Behaviour trial (novel environment) set up

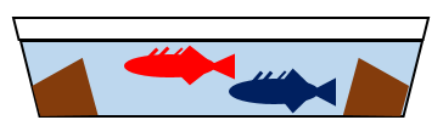

C

Unfamiliar dyads

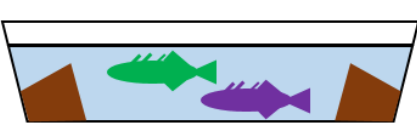

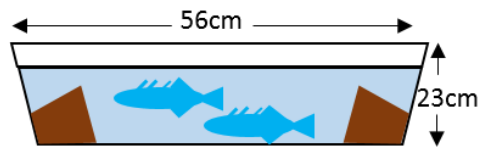

D

Familiar dyads

Post-test sample

Figure 3.1: Experimental setup for investigating the effects of recent social context and familiarity on cortisol responses in three-spined stickleback fish. (A) Individuals housed solitarily prior to experiments and tested with an unfamiliar partner (that had also been housed solitarily). (B) Individuals housed socially and tested with an unfamiliar partner. (C) Individuals housed socially and tested with that familiar partner. Dyads were tested in the novel environment tank twice and a post-test hormone sample was taken each time, a pre-test sample was also taken before the experiments ( $n=3$ samples per individual; see also Figure 3.2). Dyad partners remained the same throughout the experiments.

\section{Hormone collection and analysis}

A total of $n=216$ waterborne hormone samples were collected $(n=3$ for each individual, see Figure 3.2) and were analysed for cortisol, following published procedures (Fürtbauer et al. 2015b, a; Fürtbauer \& Heistermann 2016). Prior to the experiments, each individual was subject to two beaker habituation phases, the first occurred two days before the experiment day and the second occurred the day before the experiment day. This was done to habituate individuals to the beaker so that any increase in cortisol concentration did not reflect response to confinement. Previous research across a 5 day habituation period showed that cortisol increases during the first two days of confinement, but significantly decreases from day 3 and remains stable in subsequent beaker confinement (Fürtbauer et al. 2015b). The habituation phase process was the same as the process described for hormone collection within the experiments (habituation water samples were not stored for cortisol extraction). In brief, for each 
hormone collection (either straight from their housing tank, pre-NE sample, or immediately following an NE test, post-NE sample) fish were placed in a $150 \mathrm{~mL}$ glass beaker filled with $60 \mathrm{~mL}$ of water (same source as home tank) for 1 hour. Water samples were filtered through a net and collected in $60 \mathrm{~mL}$ plastic polypropylene bottles, stored at $-18^{\circ} \mathrm{C}$ until analysis. Solid phase extraction methods were used to extract steroids (cortisol) using Sep-Pak Plus C18 solid phase extraction cartridges and a Visiprep 12-port vacuum manifold connected to laboratory mains air source. $5 \mathrm{~mL}$ of methanol was used to remove steroids from the cartridges, which were collected in glass tubes and then evaporated under a stream of nitrogen at $38^{\circ} \mathrm{C} .250 \mu \mathrm{L}$ of assay buffer was used to re-suspend the steroids. Salimetrics saliva cortisol enzyme immunoassays were used to measure cortisol concentrations (Salimetrics LLC, State College, PA), following the manufacturer's instructions. Samples were run in duplicates and samples with a c.v. $>10 \%$ were re-measured. Assay sensitivity was $0.007 \mu \mathrm{g} / \mathrm{dL}$. Inter-assay coefficients of variation were $6.3 \%$ (high) and $14.9 \%$ (low) and intra-assay coefficient of variation were $2.7 \%$ (high) and $12.6 \%$ (low), calculated from replicates of the high- and low-quality controls across 8 plates.

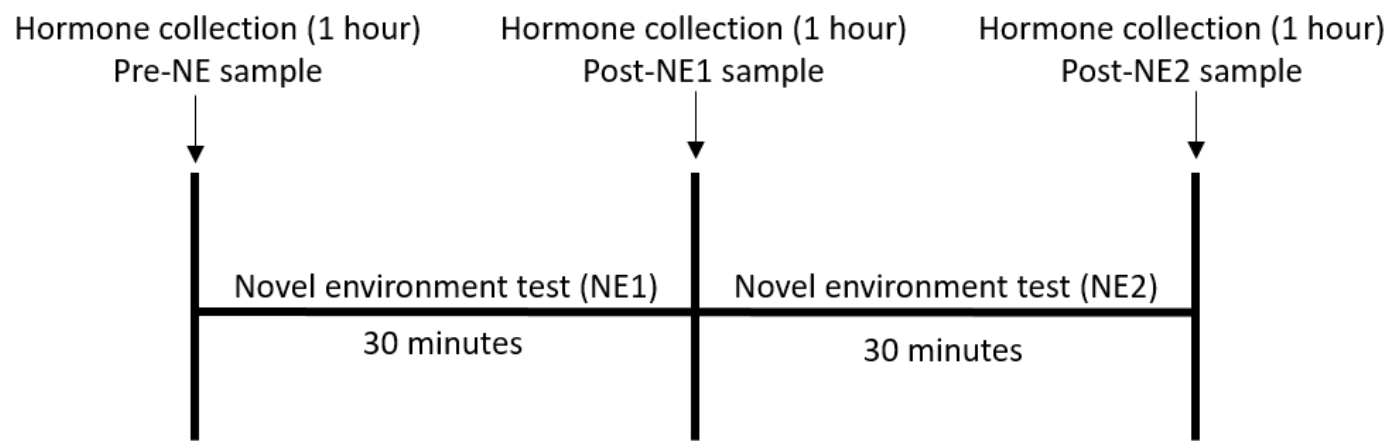

Figure 3.2: Timeline of experiment and waterborne hormone collection. Fish dyads were exposed twice to the novel environment. $\mathrm{N}=3$ waterborne hormone samples (total $\mathrm{n}=216$ ) were collected from each individual (one pre- and two post-test samples).

\section{Statistical analysis}

All statistical analysis was conducted in R (R Development Core Team 2018), using the packages lme4 and lmerTest (Bates et al. 2014). In all models, cortisol concentration was log-transformed to meet model assumptions of linear mixed models (LMMs; so that cortisol concentration fits normal distribution; Bates et al. 2014). First, to investigate potential differences in cortisol concentration between pre-NE and post- 
NE measures a linear mixed model (LMM) was used. The model (LMM1) included cortisol as the response variable and context, sex and time of sample collection (AM/PM; see Fürtbauer \& Heistermann 2016) as fixed effects. Individual ID and Dyad ID were included as random effects to control for non-independence of data. Intraclass correlation coefficient (ICC) was also calculated using the package ICC (Wolak et al. 2012) to obtain a measure of individual cortisol consistency across the three samples.

Second, to investigate effects of recent social context and familiarity on cortisol measures two LMMs were run. First, to explore the effects of recent social context on pre-NE cortisol concentrations, the model included the pre-NE cortisol samples as a response, with recent social context and sex as fixed effects (LMM2). Second, to explore the effects of recent social context and familiarity on post-NE cortisol concentrations in response to the novel environment, the model included the post-NE cortisol samples as a response, with recent social context, familiarity, sex and AM/PM as fixed effects (LMM3). Individual ID and Dyad ID were included as random effects in both models to control for non-independence of data.

Thirdly, to investigate whether partner cortisol was related to individual post-NE cortisol concentrations (i.e. cortisol coregulation) an LMM was run. Initially an interaction between partner cortisol and familiarity, and an interaction between partner cortisol and recent social housing was included (to investigate whether recent social context or familiarity affected coregulation), but these were removed due to not being significant. The final model (LMM4), included individual post-NE cortisol concentrations as the response, with partner post-NE cortisol, sex and AM/PM as fixed effects. Individual ID and Dyad ID were included as random effects to control for nonindependence of data. I also ran a linear model (LM) to investigate whether coregulation occurred in the pre-NE cortisol concentrations, with individual pre-NE cortisol as the response and partner pre-NE cortisol and sex as fixed effects (LM1).

Finally, to investigate whether changes in cortisol concentrations from pre-NE to post$\mathrm{NE}$ were predicted by (i) partner and/or (ii) individual pre-NE cortisol (baseline) concentrations an LMM was used (LMM5). The change between pre- and post-NE cortisol concentrations was fitted as the response, with partner pre-NE cortisol, individual pre-NE cortisol, recent social context, familiarity and sex as fixed effects. Individual ID and Dyad ID were included as random effects to control for non- 
independence of data. As this analysis compared individuals with different initial preNE cortisol values, there was potential for regression toward the mean to occur (Barnett 2004; Kelly \& Price 2005). Therefore, I tested for a differential effect using the test for the equality of variances in samples (equation 5; Kelly \& Price 2005).

\section{Results}

Repeatable inter-individual differences in cortisol concentrations (Prediction 1)

Waterborne cortisol concentrations ranged from $0.04-8.88 \mathrm{ng} / \mathrm{g} / \mathrm{h}(\mathrm{n}=216$; median $1.24 \mathrm{ng} / \mathrm{g} / \mathrm{h}$ ), and individuals showed strong consistency in cortisol across the three samples (ICC: 0.846, p<0.05; LMM1: p<0.001; Table 3.1).

Was the novel environment perceived as a stressor? (Prediction 2)

Individual cortisol concentrations did not differ significantly between pre-NE and post-NE measures (see Table 3.1; Figure 3.3), suggesting that individuals did not perceive the novel environment as a stressor. Pre-NE cortisol concentrations were not affected by recent social context (LMM2; Table 3.2) and post-NE cortisol concentrations were not affected by recent social context or familiarity (LMM3; Table 3.2). Males had significantly lower pre- and post-NE cortisol concentrations (LMM2, LMM3; Table 3.2). I also found no effect of time of day (morning or afternoon sample) on cortisol concentration (LMM1; Table 3.1).

Table 3.1: Changes in waterborne cortisol concentrations (ng/g/h) across pre-NE and post-NE measures for $n=72$ stickleback fish. Fish ID and Dyad ID were included as random effects. Statistically significant results are shown in bold.

\begin{tabular}{llcl}
\hline Model & Predictor Variable & Estimate \pm s.e. & p-value \\
\hline LMM1 & & & \\
Log(Cortisol ng/g/h) & & & \\
Fixed Effects & Intercept & $0.31 \pm 0.12$ & \\
& Context NE1 & $-0.03 \pm 0.11$ & 0.764 \\
& Context NE2 & $0.07 \pm 0.11$ & 0.540 \\
& Sex Male & $-0.81 \pm 0.19$ & $<\mathbf{0 . 0 0 1}$ \\
& Time PM & $0.10 \pm 0.14$ & 0.490 \\
Random Effects & & $\boldsymbol{\chi}^{\mathbf{2}}$ & $\boldsymbol{p}$-value \\
& Fish ID & 70.29 & $\mathbf{< . 0 0 1}$ \\
& Group ID & 41.47 & $\mathbf{< 0 . 0 0 1}$ \\
\hline
\end{tabular}




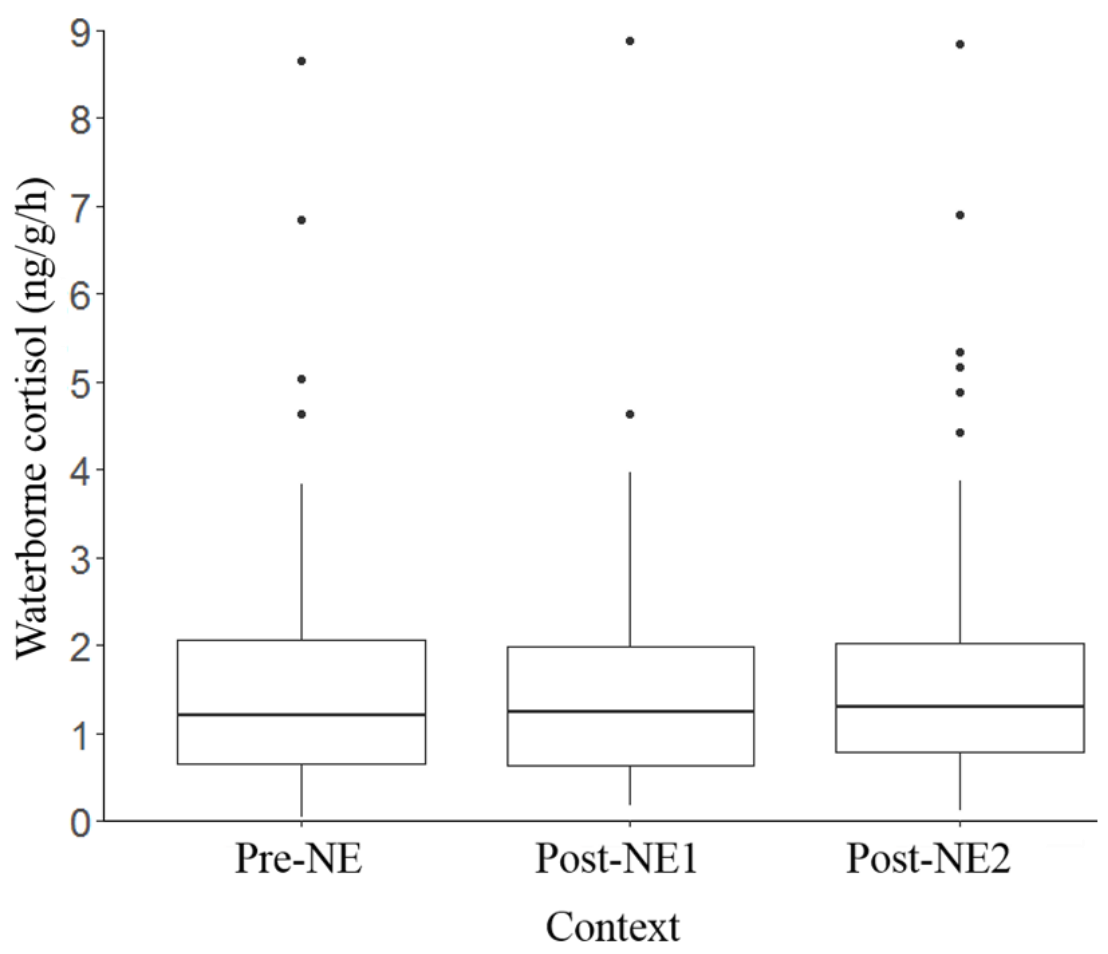

Figure 3.3: Waterborne cortisol concentrations in $n=72$ stickleback fish. For each fish, samples were collected before (pre-NE) and after the first (post-NE1) and second (post-NE2) novel environment test. Note that untransformed cortisol concentrations are shown. Box and whiskers show average value, first and third quartile and minimum and maximum values. Data points that fall outside the quartiles are outliers represented by dots.

Table 3.2: Factors affecting pre-NE (LMM2) and post-NE (LMM3) waterborne cortisol concentrations $(\mathrm{ng} / \mathrm{g} / \mathrm{h})$ for $\mathrm{n}=72$ stickleback fish. Fish ID and Dyad ID were included as random effects. Statistically significant results are shown in bold.

\begin{tabular}{lllr}
\hline Model & Predictor Variable & Estimate \pm s.e. & p-value \\
\hline LMM2 & & & \\
Pre-NE Log(Cortisol ng/g/h) & & & \\
Fixed Effects & Intercept & $0.43 \pm 0.16$ & \\
& Housing solitary & $-0.50 \pm 0.25$ & 0.050 \\
& Sex Male & $-0.63 \pm 0.2 .5$ & $\mathbf{0 . 0 1 6}$ \\
Random Effects & & $\chi^{2}$ & $\boldsymbol{p}$-value \\
& Group ID & 6.73 & $\mathbf{0 . 0 3 5}$ \\
LMM3 & & & \\
Post-NE Log(Cortisol ng/g/h) & & & \\
Fixed Effects & Intercept & $0.59 \pm 0.19$ & \\
& Housing solitary & $-0.12 \pm 0.23$ & 0.646 \\
& Unfamiliar & $-0.09 \pm 0.24$ & 0.720 \\
& Sex Male & $-0.87 \pm 0.20$ & $<\mathbf{0 . 0 0 1}$ \\
Random Effects & & $\chi^{2}$ & $\boldsymbol{p}$-value \\
& Fish ID & 54.77 & $<\mathbf{0 . 0 0 1}$ \\
& Group ID & 22.36 & $<\mathbf{0 . 0 0 1}$ \\
\hline
\end{tabular}


Cortisol concentrations of dyad partners were unrelated pre- and post-NE (LMM4-5; Table 3.3) and there was no interaction between dyads' cortisol concentrations and recent social context or familiarity (Appendix 1 Table 8.1), indicating an absence of cortisol coregulation. Whilst individual (focal fish) pre-NE cortisol concentrations were positively correlated with individual cortisol change; partner pre-NE was not correlated with cortisol change (Appendix 1 Table 8.2; Figure 3.4). This means that individual pre-NE cortisol concentration predicted cortisol change, but partner pre-NE did not.

Table 3.3: Summary of LMMs exploring potential cortisol coregulation in stickleback fish dyads ( $\mathrm{n}=36$ dyads) for pre- and post-NE cortisol measurements. Note, both cortisol and partner cortisol were log transformed (see methods for details). Fish ID and Dyad ID were included as random effects. Statistically significant results are shown in bold.

\begin{tabular}{|c|c|c|c|c|}
\hline Model & $\begin{array}{l}\text { Response } \\
\text { Variable } \\
\end{array}$ & $\begin{array}{l}\text { Predictor } \\
\text { Variable } \\
\end{array}$ & Estimate \pm s.e. & p-value \\
\hline $\begin{array}{l}\text { LMM4 } \\
\text { Post-NE }\end{array}$ & Cortisol (ng/g/h) & $\begin{array}{l}\text { Intercept } \\
\text { Partner cortisol } \\
\text { Sex Male } \\
\text { Time: PM }\end{array}$ & $\begin{array}{r}0.49 \pm 0.14 \\
0.05 \pm 0.08 \\
-0.91 \pm 0.20 \\
-0.20 \pm 0.18\end{array}$ & $\begin{array}{r}0.506 \\
<\mathbf{0 . 0 0 1} \\
0.267\end{array}$ \\
\hline Random Effects & & $\begin{array}{l}\text { Fish ID } \\
\text { Group ID }\end{array}$ & $\begin{array}{l}\chi^{2} \\
55.34 \\
49.83 \\
\end{array}$ & $\begin{array}{c}\text { p-value } \\
<0.001 \\
<0.001 \\
\end{array}$ \\
\hline $\begin{array}{l}\text { LM1 } \\
\text { Pre-NE }\end{array}$ & Cortisol (ng/g/h) & $\begin{array}{l}\text { Intercept } \\
\text { Partner cortisol } \\
\text { Sex Male } \\
\text { Time PM }\end{array}$ & $\begin{array}{c}0.29 \pm 0.14 \\
0.08 \pm 0.11 \\
-0.74 \pm 0.26 \\
0.099 \pm 0.240\end{array}$ & $\begin{array}{l}0.499 \\
\mathbf{0 . 0 0 5} \\
0.740 \\
\end{array}$ \\
\hline
\end{tabular}

\section{Plasticity in cortisol concentrations (Predictions $6 \& 7$ )}

Individuals' pre-NE cortisol concentrations were negatively correlated with the change in cortisol concentrations between pre- and post-NE1 (estimate \pm s.e. $=-0.80 \pm 0.13$, $\mathrm{p}<0.001$; Figure 3.4B), indicating that individuals baseline cortisol predicts cortisol plasticity, with individuals with a higher baseline cortisol showing a greater decrease in response to the novel environment. Whilst overall there were no significant differences between the sampling points (Figure 3.3), some individuals showed a 
decrease, whilst others showed an increase (Figure 3.4B). A post hoc analysis showed that housing condition did not affect individual's cortisol change (Appendix 1 Table 8.3; Figure 3.4B). As this analysis compared individuals with different initial baseline cortisol values, there was potential for regression toward the mean to occur (Barnett 2004; Kelly \& Price 2005). Using the equation described by Kelly \& Price (2005), I tested for a differential effect using the test for the equality of variances in paired samples (equation 5; Kelly \& Price 2005). I found evidence for a differential effect ( $T$ $=3.3$, df $=71, \mathrm{p}<0.05)$, which suggests that the observed relationship between individuals baseline and cortisol change in response to the novel environment could be a statistical rather than actual relationship between the variables (Barnett 2004; Kelly \& Price 2005). Therefore, I constructed adjusted values using the equation described by Kelly \& Price (2005) and using these values individuals pre-NE cortisol concentrations were non significantly negatively correlated with the change in cortisol between pre- and post-NE1 (estimate \pm s.e. $=-0.16 \pm 0.14, \mathrm{p}=0.249$; Figure 3.5).

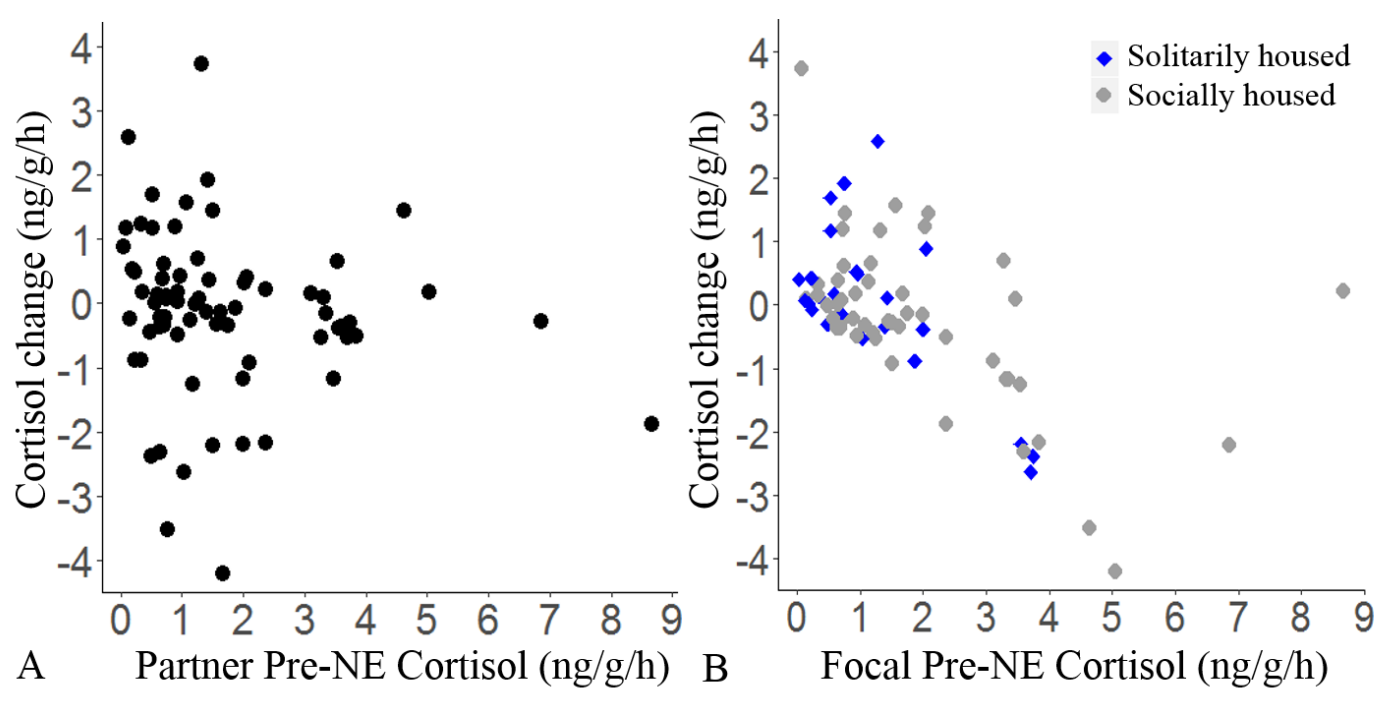

Figure 3.4: Correlation between (A) partner and (B) individual Pre-NE cortisol concentrations $(\mathrm{ng} / \mathrm{g} / \mathrm{h})$ and the change in cortisol concentrations between Pre-NE and the first novel environment test (post-NE1), $n=72$. 


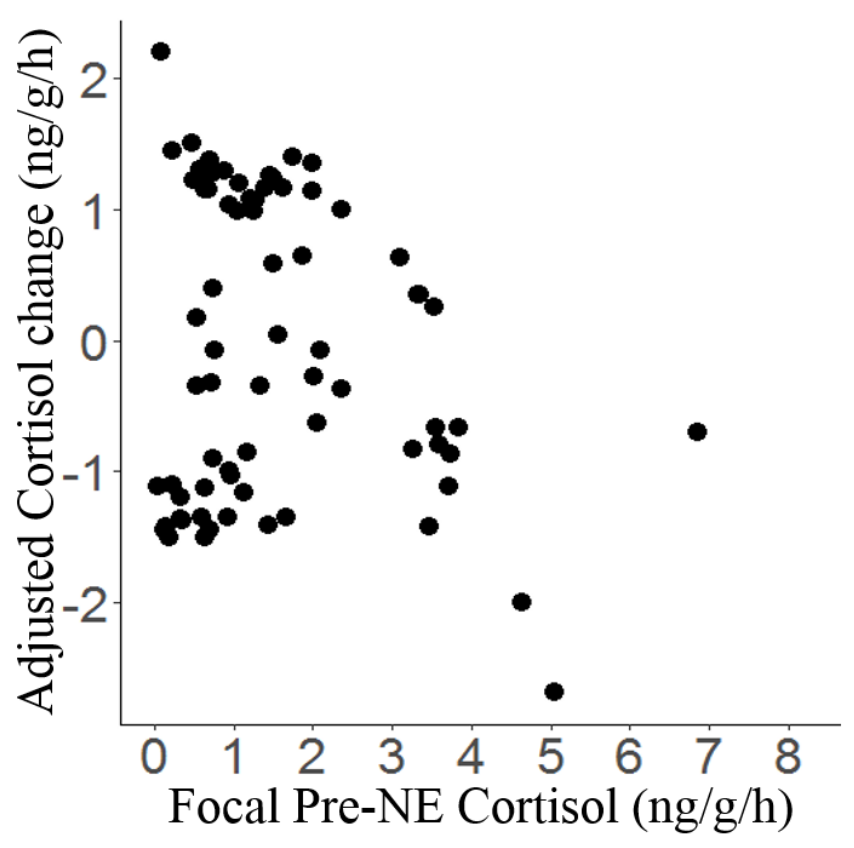

Figure 3.5: Correlation between individual Pre-NE cortisol concentrations (ng/g/h) and the adjusted for regression effects change in cortisol concentrations between Pre$\mathrm{NE}$ and the first novel environment test (post-NE1), $\mathrm{n}=72$.

\section{Discussion}

Whilst research investigating cortisol coregulation in humans has increased over the last decade, studies tend to focus on dyads with strong attachment bonds, such as romantic couples cohabiting (Saxbe \& Repetti 2010; Papp et al. 2013) or mothers and offspring (Neu et al. 2008; Papp et al. 2009; Mörelius et al. 2015), raising the question if dyad partners need to be familiar for coregulation to occur. Fish models are being used increasingly in endocrinological research due to the similarities between the hypothalamic-pituitary-interrenal (HPI) axis and the hypothalamic-pituitary-adrenal (HPA) axis in humans (Oliveira \& Galhardo 2009; Pankhurst 2011). Recent research on sticklebacks has shown evidence for cortisol coregulation in a stressful context (Fürtbauer \& Heistermann 2016). Building upon this work, the present study aimed to explore whether familiarity/recent social context affects cortisol coregulation. I also aimed to explore repeatability and plasticity in inter-individual differences in cortisol concentrations and whether there were any effects of familiarity/recent social context.

I did not find evidence of cortisol coregulation in fish dyads in response to a novel environment, which may be explained by the fact that the novel environment was not 
perceived as a stressor, i.e. cortisol concentrations did not significantly change from pre-NE to post-NE (see Figure 3.3). Whilst previous work has found that cortisol concentrations significantly increased in fish exposed to a shared open field environment (Fürtbauer \& Heistermann 2016), the present study used a 'novel environment' including two shelters (flower pots). These shelters could have potentially reduced the perceived risk, resulting in the lack of an acute physiological stress response; a potential requirement for coregulation to occur.

Human couples who perceive their relationship as 'stressful' (i.e. lack of perceived spousal support and experiencing disagreements) exhibit saliva cortisol coregulation (Liu et al. 2013; Saxbe et al. 2015). In stickleback fish, waterborne cortisol concentration's increase in response to open field environments and under these conditions cortisol coregulation within familiar dyads occurs (Fürtbauer \& Heistermann 2016). The results from this study and work on humans suggests that a 'stressful' environment (i.e. one where individuals show an increase in HPA/I axis activity) may affect presence and strength of coregulation (Saxbe \& Repetti 2010; Liu et al. 2013; Fürtbauer \& Heistermann 2016). Coregulation in 'stressful' environments could occur between individuals as a way of coping with challenges and/or changes in the environment (Saxbe \& Repetti 2010; Liu et al. 2013; Fürtbauer \& Heistermann 2016). Given that I did not find the fish perceived the novel environment as a stressor, it is not unsurprising that I find no evidence for cortisol coregulation between pairs of fish. Further research comparing whether the same dyads coregulate under both 'stressful' and 'non-stressful' conditions is therefore now needed to investigate the full conditions under which coregulation occurs in non-human animals.

Investigation into whether individual pre-NE cortisol measure predicts cortisol change/coregulation revealed that, whilst there was no population level change in cortisol, individuals with higher pre-NE cortisol show decrease in cortisol (and showed a greater level of change; greater plasticity) whilst individuals with lower baseline cortisol either showed no change or slight increases in cortisol levels (see Figure 3.4). These results assess the slope of individual plasticity and results suggest that baseline measures may be an important factor influencing flexibility (Taff \& Vitousek 2016; Stedman et al. 2017). Perhaps this finding indicates that individuals with a higher pre$\mathrm{NE}$ cortisol concentration have a more flexible physiological response to environmental change and regulate their cortisol accordingly. Whilst there were no 
significant changes, perhaps these individuals perceived the housing as slightly more 'stressful' (i.e. perhaps these individuals experienced high aggression with housing partners or found the housing area too small Ashley 2007; Keck et al. 2015).

On a functional level, this increased physiological flexibility of individuals with higher baseline measures could have adaptive significance as it suggests that these individuals may be able to better adapt to changing environments (Piersma \& Drent 2003; Taff \& Vitousek 2016). In my study, the adaptive flexibility to the novel environment would be to decrease cortisol as a response to a non-threatening novel environment, this is adaptive as prolonged, elevated levels of cortisol have negative consequences on growth and feed intake (Nelson 2011). Indeed, if individuals that have higher baseline/pre-environmental change cortisol concentrations are more flexible in general, we would expect that these individuals would show a greater increase (greater slope of plasticity) in response to a physiological stressor (i.e. an open environment or in response to a predator) as well as a greater decrease when there is not a physiological stressor (Taff \& Vitousek 2016). Therefore, future studies should investigate individuals' responses to environmental changes which cause a physiological response (i.e. increase in cortisol post-environmental change) and those that do not and whether links between baseline (i.e. pre-stressor) measures and plasticity are consistent across contexts.

\section{Conclusion}

This study did not find evidence for cortisol coregulation in a non-stressful environment suggesting that coregulation occurs only under certain (stressful) environmental conditions which is in line with research on humans (Saxbe \& Repetti 2010; Liu et al. 2013; Fürtbauer \& Heistermann 2016). The absence of coregulation prevented me from investigating familiarity effects on cortisol coregulation, providing avenues for future research. Cortisol concentrations did not significantly change from pre- to post-NE, and neither pre-NE or post-NE cortisol concentrations were not affected by recent social context or familiarity. Additionally, individuals pre-NE (i.e. their baseline) predicted their level of endocrine plasticity, suggesting that baseline measure may be an important factor influencing flexibility (Taff \& Vitousek 2016; Stedman et al. 2017), and indicating that perhaps individuals with higher baseline/preNE cortisol may have a more flexible physiological response to environment change 
and regulate their cortisol accordingly (Piersma \& Drent 2003). Overall, the findings of this study suggest that whilst inter-individual differences in cortisol concentrations persist, when a novel environment is not physiologically stressful (i.e. no increase in HPA axis activity) there are no effects of recent social context on familiarity and that cortisol coregulation does not occur between dyads. These results suggest that in order to better understand the effects of recent social context and familiarity on cortisol responses, future research should investigate dyadic cortisol responses across a range of changing (stressful and non-stressful) environments to better understand what physical and social conditions affect cortisol responses. 


\title{
Chapter 4: Effects of recent housing condition, familiarity and hypothalamic-pituitary-interrenal (HPI) axis activity on behavioural responses in dyadic social contexts in three-spined stickleback fish (Gasterosteus aculeatus)
}

The work presented in this chapter formed part of a conference poster presentation (Appendix 4):

Fry, A., \& Fürtbauer, I. (2019) The effects of familiarity on repeatability of behavior in pairs of three-spined sticklebacks. New Frontiers in the Study of Animal Behaviour (ASAB), Liverpool, UK.

\begin{abstract}
Recent housing condition (socially versus solitarily housed) and familiarity with a partner can affect behaviour as well as social interactions which occur frequently within groups of animals. Effects of social context on repeatability of behaviour have been studied in non-social contexts; however, it remains unknown whether recent social context and familiarity affect repeatability and behavioural responses also in social contexts. Furthermore, behaviour can also be linked to physiological measures and coping styles (i.e. a set of behaviour and physiological traits) are often found in stressful contexts, however, potential effects of social context on coping styles are yet to be explored. Using dyads of three-spined stickleback fish (Gasterosteus aculeatus), I investigate effects of recent social conditions on short-term repeatability and behavioural responses when exposed twice to a novel environment. I further test whether behavioural responses are linked to physiological stress levels, using waterborne cortisol analysis. Individual behaviour measures (activity, individual shelter use, and proportion of time in shelter with partner) but not the dyadic behaviour (inter-dyadic distance) were repeatable. Familiarity did not affect repeatability in either individual or dyadic behaviour measures. Activity levels decreased, individual shelter and inter-dyadic distance increased across behaviour trials. Shared shelter use increased in the second trial for unfamiliar social dyads, perhaps a result of interest in a new social partner. Waterborne cortisol concentrations were negatively correlated with activity (total distance travelled), showing individuals with higher hypothalamicpituitary-interrenal (HPI) axis activity had lower activity levels, providing evidence of 'coping styles'. Taken together these results suggest that recent social context does not affect the repeatability of behaviour or changes in individual behaviours in dyadic social contexts. Overall, these findings therefore suggest that the current social/ecological environment and individual personalities could explain behaviour variation better than previous environments. However, the results suggest that familiarity can affect specific behaviour in future social contexts.
\end{abstract}




\section{Introduction}

Consistent individual differences in behaviour across time and/or contexts (i.e. animal personality) is widespread across the animal kingdom (Sih 2004; Bell et al. 2009; Stamps \& Groothuis 2010). For example, fish of several species show repeatability in activity/distance travelled (Marras et al. 2011; Poulin et al. 2012; Taylor \& Cooke 2014; Fürtbauer et al. 2015b; O’Neill et al. 2018) and time spent in shelter/latency to emerge from hiding (O’Neill et al. 2018; Roy \& Bhat 2018). An increasing amount of research has compared behaviour of individuals when alone to when in a social pair or group, with particular focus on fish due to the natural formation of social shoals (Bierbach et al. 2017; Guayasamin et al. 2017), and showing consistency in behaviour across non-social and social contexts (reviewed by Webster \& Ward 2011).

Social interactions frequently occur within groups of animals, and it is well established that social interactions can affect activity levels (Cordeiro et al. 2018; StrandburgPeshkin et al. 2018), aggressive behaviour (Sokolowski 2010; Sachser et al. 2013) and avoidance behaviour (Stins et al. 2011). Whilst research has shown that behaviour can change in response to social contexts (Laland \& Williams 1998; Kurvers et al. 2013; King et al. 2015; Koski \& Burkart 2015; Fürtbauer \& Fry 2018), there remains little research on repeatability of behaviour within pairs over time, which represents a more natural social context for social animals, as opposed to testing individuals within nonsocial contexts or across non-social and social contexts.

Previous social context (e.g. whether individuals have been in groups or in isolation), has also been shown to affect an individual's behaviour in groups in relation to shoaling decisions (Webster \& Hart 2006), risk-taking (van Oers et al. 2005) and leadership (Laland \& Williams 1998). Individual isolation leads to increased locomotion in open field environments in zebrafish (Danio rerio Shams et al. 2018), rats (Rattus spp. Varlinskaya et al. 1999), diary calves (Bos tarus Jensen et al. 1999; Chua et al. 2002), tigers (Panthera tigris De Rouck \& Law 2005) and domesticated dogs (Canis lupus familiaris Hetts et al. 1992). Housing individuals in isolation or socially has been shown to affect certain behaviours, with abnormal behaviours having been shown to occur in individuals exposed to challenges or novel environments (Mertens \& Unshelm 1996; Costa et al. 2016; White et al. 2017). For example, in a reversal learning response to novel objects in diary calves individually housed calves were slower to approach the novel object and were unable to correctly re-learn the task 
whilst the socially housed calves could (Meagher et al. 2015). In tigers, individuals housed in pairs spent more time performing 'natural' behaviours, whereas individually housed tigers spent more time auto-playing (De Rouck \& Law 2005). In horses 'stress' behaviours were observed more frequently in individually housed compared to social house individuals (Visser et al. 2008). Additionally, in dairy calves (Jensen et al. 1999), rats, and mice (Olsson \& Westlund 2007) individuals housed in isolation showed more exploratory behaviour to a novel environment than socially housed individuals.

Furthermore, recent social context can have 'carry over' effects and influence repeatability in behaviour across non-social and social contexts (Jolles et al. 2016). Housing three-spined stickleback (Gasterosteus aculeatus) fish either solitarily, partly solitarily or socially prior to an individual risk-taking task (i.e. time to emerge out of deep water into shallow water) showed that the recent social context influenced the repeatability of behaviour, with solitary housed individuals showing higher repeatability than socially housed fish (Jolles et al. 2016). Also, previous partner personality (i.e. whether partner is bold or shy) in combination with focal fish personality has been shown to affect individuals' own behaviour in a novel environment, with bold fish paired with a bold partner spending more time out of cover (Jolles et al. 2014).

In addition to recent social context, familiarity of interacting individuals can affect behaviour (including both repeatability and behavioural changes), with individuals behaving differently when with unfamiliar compared to when with familiar group members (reviewed by Ward \& Hart 2003). Plasticity in behaviour, such as decreasing exploration and remaining close to group members when in a new environment can have advantages including information transfer (e.g. knowing where food resources are) and group protection from predators (Manning \& Dawkins 1998; Morrell et al. 2008). Individuals of numerous species choose to associate with familiar over unfamiliar partners (Griffiths \& Magurran 1997; Dzieweczynski et al. 2012; Thünken et al. 2016; Keller et al. 2017) and familiar dyads habituate faster to novel environments (Valero et al. 2009; Keller et al. 2017). In shoals of three-spined stickleback fish, for example, individuals stay closer to conspecifics (Atton et al. 2014), and show more explorative behaviour in response to a novel environment or novel object test when with a familiar individual (Chivers et al. 1995; Liebgold \& 
Dibble 2011; Galhardo et al. 2012). Familiarity is important in animal groups; it can help mediate a stable hierarchy, decisions, influence mate-choice decision, and even reduce aggressive behaviour (Atton et al. 2014). Investigating the effects of familiarity on individual behaviour is important to better understand how individuals respond to changing social environments; particularly important in fission-fusion systems with variable group members, and this can have implications for research in commercial settings where individuals tend to be housed in smaller groups (Webster \& Ward 2011). Fish shoals are a good example of this, with individuals constantly joining and leaving shoals (Couzin 2006). Overall, whilst it is clear that recent social housing and personality of partners can affect future individual behaviour (Jolles et al. 2014, 2016), it remains unknown whether previous social experience (e.g. recent housing condition) and familiarity with a partner affects behaviour in social contexts.

Behavioural responses shown in social and non-social contexts may be underpinned by changing physiology in response to the change in environmental conditions (Nelson 2011). In response to an environmental stressor the hypothalamic pituitary adrenal/interrenal (HPA/I) axis is activated, which stimulates a cascade of internal reactions where cortisol is produced (Alsop \& Vijayan 2009; Nelson 2011). Coping styles are defined as coherent sets of physiological and behavioural responses, with two different coping styles commonly observed: reactive (behaviourally less active, less risk taking and higher HPA/I) and proactive (behaviourally more active, more risk taking and lower HPA/I) individuals (Koolhaas et al. 1999, 2010; Edwards \& Casto 2013; Castanheira et al. 2017). Coping styles have been described across vertebrate taxa and are particularly well studied in fish, including three-spine sticklebacks (Fürtbauer et al. 2015b), rainbow trout (Oncorhynchus mykiss Øverli et al. 2004), brown trout (Salmo trutta Brelin et al. 2005), Siamese fighting fish (Betta splendens Verbeek et al. 2008) and halibut (Hippoglossus hippoglossus Kristiansen \& Fernö 2007). However, it is currently unknown whether individual coping styles can be affected by the previous (i.e. recent social housing) or current (i.e. familiarity) social context.

In this study, I investigate whether recent social context (i.e. solitarily or socially housed; see details below) and current social context (i.e. familiarity) affect behavioural responses in dyads of three-spined stickleback fish exposed twice to a new environment. Three-spined stickleback fish are excellent models to explore 
behavioural responses and social interactions as they are naturally social species that usually shoal together (Huntingford \& Ruiz-Gomez 2009). They are also a relatively robust species, and are able to be housed either individually or in groups in a laboratory setting, which allows easy investigation into effects of recent housing conditions on individual behaviour (Huntingford \& Ruiz-Gomez 2009; Jolles et al. 2016). Threespined stickleback fish have been used in previous dyadic experiments (Fürtbauer \& Heistermann 2016) along with group behaviour experiments on familiarity (Ward et al. 2004; Ward \& Hart 2005) and recent social conditions (Jolles et al. 2016).

Firstly, I investigate whether individual behaviour measures (total distance travelled, individual shelter use and proportion of individual time in shelter spent with partner) and dyadic behaviour (inter-dyad distance) were repeatable across trials and, whether repeatability of behaviours was affected by recent housing contexts and/or familiarity with a partner during trials. Based on previous research showing repeatable behaviour in solitary and partially solitary but not socially housed individuals (Jolles et al. 2016), I predicted that (i) solitary housed individuals would show repeatability in individual behaviours. Given that stickleback fish remain closer to familiar over unfamiliar shoal members (Ward et al. 2004; Ward \& Hart 2005; Atton et al. 2014), I further predicted that (ii) inter-dyadic distance would be repeatable in familiar but not in unfamiliar dyads.

Secondly, I investigated factors that predicted behaviour including, recent social housing, familiarity with partner in the behaviour test, cortisol measures and tested for potential changes in behaviour across the two trials. Given the extensive evidence of 'coping styles' in fish, I predicted that (iii) individuals with higher HPI axis activity would be less active and spend more time in shelter. Also as 'exploration' and 'shyness' decrease in individuals exposed to repeated trials of the same environment (Ward et al. 2008; Bolivar 2009; Matsunaga \& Watanabe 2010), I predicted that (iv) individuals would be less active and hide less in the second trial. As this study enabled me to investigate both the effects of recent housing and familiarity, I predicted that (v) familiar dyads would be more active, remain closer together and spend more time together in shelter with their partner compared to unfamiliar dyads. This is based on previous research finding that familiar individuals are more similar in their behaviour, remain closer to each other and explore a novel environment more compared to unfamiliar dyads (Liebgold \& Dibble 2011; Galhardo et al. 2012; Atton et al. 2014). 
As stickleback fish are naturally shoaling fish (Huntingford \& Ruiz-Gomez 2009) and socially housed individuals were paired with another individual throughout (compared to solitary housed individuals), I predicted that socially housed dyads would (vi) remain closer to their partner and spend more time in shelter with that partner. Additionally, given that even with limited familiarity (i.e. familiarity from a previous group experiment) groups of stickleback fish are similar in behaviours (Jolles et al. 2018), I predicted that (vii) unfamiliar individuals would increase time spent together and that inter-dyadic distance would decrease in the second trial (as a result of familiarity gained in the first trial) compared to the first trial.

\section{Methods}

\section{Subjects and housing}

$\mathrm{N}=76$ stickleback fish ( $\mathrm{n}=56$ females, $\mathrm{n}=20$ males), sourced from a wild, commercially bred population through CarpCo, Kent, England (www.carpco.co.uk/) were used for the experiments. Prior to experiments fish were either housed in social pairs or solitarily ( $\mathrm{n}=24$ dyad tanks, $\mathrm{n}=24$ solitary tanks). All fish were tested in dyads and as a result of being housed socially or solitarily within the experiments dyads were either familiar or unfamiliar. Therefore, experimental dyads could be familiar and previously housed socially (i.e. housed together), unfamiliar and previously housed socially (i.e. housed with a different individual) or unfamiliar and previously housed solitary. The experiment consisted of two exposures to a novel environment, which were filmed for behavioural extraction (see below for full details). $\mathrm{N}=3$ waterborne hormone samples were collected for each individual and were analysed for cortisol concentrations. For full details of experimental procedures, hormone collection and analysis, see Chapter 3.

\section{Video analysis and behavioural measures}

A total of $n=72$ behavioural trials were filmed using a Panasonic HDC-SD60 video camera ( $n=2$ behavioural trials for each dyad). Using the high-resolution imagetracking software IdTracker, which allows individual identification of multiple individuals (Pérez-Escudero et al. 2014), x, y coordinates of fish movements were extracted. These coordinates were used to calculate the following behaviour measures; (i) total distance travelled (m), (ii) total individual shelter use (mins), (iii) proportion of individual time in shelter with partner (\%) and (iv) inter-dyad distance (m). 


\section{Statistical analysis}

All statistical analysis was conducted in R (R Development Core Team 2018) using packages lme4 and lmerTest (Bates \& Pinheiro 2000; Bates et al. 2014) for linear mixed model (LMM) and generalised linear mixed model (GLMM) analysis. First, repeatability in all four behaviour measures (i-iv; see above) across trials one and two were investigated. LMMs were used for distance travelled and individual shelter use, and a GLMM was used for proportion of individual time in shelter with partner (Appendix 2 Table 8.4). A linear model was used to test repeatability in inter-dyad distance (Appendix 2 Table 8.5). Inter-dyad distance was log transformed to meet model assumptions (i.e. so that inter-dyad distance fitted normal distrubtion; Bates et al. 2014). To investigate the effect of recent social housing and familiarity on repeatability of behaviour, an interaction between trial and (i) recent housing (social or solitary) and (ii) familiarity with a partner was initially fitted. This interaction was removed in all models testing for repeatability due to non-significance. The final models included the behaviour measure in trial two as the response, and the behaviour in trail one and sex as fixed effects. Dyadic ID was included as a random effect in the LMM/GLMM models. Second, predictors of behaviours and potential changes in behaviour between trial one and trial two were investigated. LMMs were used for distance travelled, individual shelter use, and inter-dyad distance (Appendix 2 Table 8.6). Initially, interactions between trial and (i) previous housing and (ii) familiarity were included to test if behaviour was dependent on housing and familiarity; this was removed as it was not significant. A GLMM was used to explore predictors and potential changes in the proportion of individual time in shelter with partner between trials (Appendix 2 Table 8.6), which was square root transformed to meet model assumptions (Bates et al. 2014). As a significant interaction between trial and familiarity was found in this model, post hoc analysis was conducted to determine the direction of the significant relationship. Two separate models (GLMM 3-4; Table 4.2) were run for familiar and unfamiliar contexts, using the same previously established significant fixed effects as the original GLMM (trial and sex).

In the behaviour models (distance travelled, individual shelter use and proportion of individual time in shelter with partner) cortisol concentration was included to investigate the relationship between behaviour and physiology (i.e. coping styles Koolhaas et al. 1999). Average focal cortisol concentration was used given that 
cortisol was consistent across the three measures (Chapter 3). Variables included in the final models are shown in Table 4.1 below. Where possible all models included sex to control for sex differences (McQuillan et al. 2003; Kudielka \& Kirschbaum 2004).

Table 4.1: Variables included in final models investigating repeatability, predictors of behaviour and potential changes in the four behaviour measures across two trials. Random effect(s) were included in all (G)LMMs to control for the non-independence of data. Sex was controlled for in individual behaviour models.

\begin{tabular}{|c|c|c|c|c|}
\hline $\begin{array}{l}\text { Research } \\
\text { question }\end{array}$ & Model & $\begin{array}{l}\text { Response } \\
\text { Variable }\end{array}$ & Fixed Variables & $\begin{array}{l}\text { Random } \\
\operatorname{effect}(s)\end{array}$ \\
\hline \multirow{4}{*}{ Repeatability } & LMM1 & $\begin{array}{l}\text { Distance } \\
\text { travelled trial } 2\end{array}$ & \multirow{3}{*}{$\begin{array}{l}\text { Behaviour in trial } 1 \text {, } \\
\text { sex }\end{array}$} & \multirow{3}{*}{ Dyad ID } \\
\hline & LMM2 & $\begin{array}{l}\text { Shelter use trial } \\
2\end{array}$ & & \\
\hline & GLMM1 & $\begin{array}{l}\text { \% Together in } \\
\text { shelter trial } 2\end{array}$ & & \\
\hline & LM1 & $\begin{array}{l}\text { Inter-dyad } \\
\text { distance trial } 2\end{array}$ & $\begin{array}{l}\text { Behaviour in trial } 1 \text {, } \\
\text { sex }\end{array}$ & \\
\hline \multirow{4}{*}{$\begin{array}{l}\text { Potential } \\
\text { changes }\end{array}$} & LMM3 & Distance & \multirow{3}{*}{$\begin{array}{c}\text { Trial }^{*} \text {, housing*, } \\
\text { familiarity*, cortisol } \\
\text { sex }\end{array}$} & \multirow{3}{*}{$\begin{array}{c}\text { Dyad ID, } \\
\text { Fish ID }\end{array}$} \\
\hline & LMM4 & $\begin{array}{l}\text { travelled } \\
\text { Shelter }\end{array}$ & & \\
\hline & GLMM2** & $\begin{array}{l}\text { \% Together in } \\
\text { shelter }\end{array}$ & & \\
\hline & LMM5 & $\begin{array}{l}\text { Inter-dyad } \\
\text { distance }\end{array}$ & $\begin{array}{l}\text { Trial*, housing*, } \\
\text { familiarity* }\end{array}$ & Dyad ID \\
\hline
\end{tabular}

*Categorical effect

*For post hoc analysis see Table 2

\section{Results}

Total distance travelled ranged from $0.03 \mathrm{~m}-130.60 \mathrm{~m}$ (average $\pm \mathrm{SD}=40.50 \mathrm{~m} \pm$ $32.80 \mathrm{~m}$ ) and individual shelter use ranged from 0.00 mins -30.00 mins (average \pm $\mathrm{SD}=12.37$ mins \pm 10.14 mins). Proportion of time in shelter with partner ranged from $0 \%-100 \%$ (average $\pm \mathrm{SD}=8.27 \% \pm 8.20 \%$ ) and inter-dyad distance ranged from $0.03 \mathrm{~m}-0.50 \mathrm{~m}$ (average $\pm \mathrm{SD}=0.18 \mathrm{~m} \pm 0.08 \mathrm{~m}$ ). 


\section{Repeatability in behaviour}

Total distance travelled (estimate \pm s.e. $=0.35 \pm 0.07, \mathrm{p}<0.001 ;$ Figure 4.1A; Appendix 2; Table 8.4; LMM1), individual shelter use (estimate \pm s.e. $=0.38 \pm 0.17, p=0.032$; Figure 4.1B; Appendix 2; Table 8.4; LMM2) and proportion of individual time in shelter with partner (estimate \pm s.e. $=0.29 \pm 0.15, p=0.049$; Figure 4.1C; Appendix 2; Table 8.4; GLMM1) were significantly positivity correlated across trials indicating repeatability in individual behaviour measures. Inter-dyad distance was not significantly correlated across trials (Figure 4.1D; Appendix 2; Table 8.5; LM1), indicating a lack of repeatability in the dyadic behaviour measure.

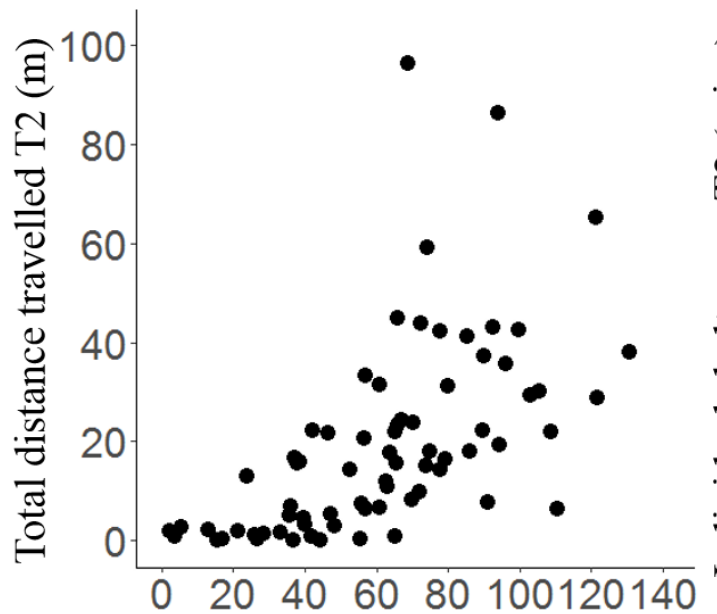

A Total distance travelled T1 (m)

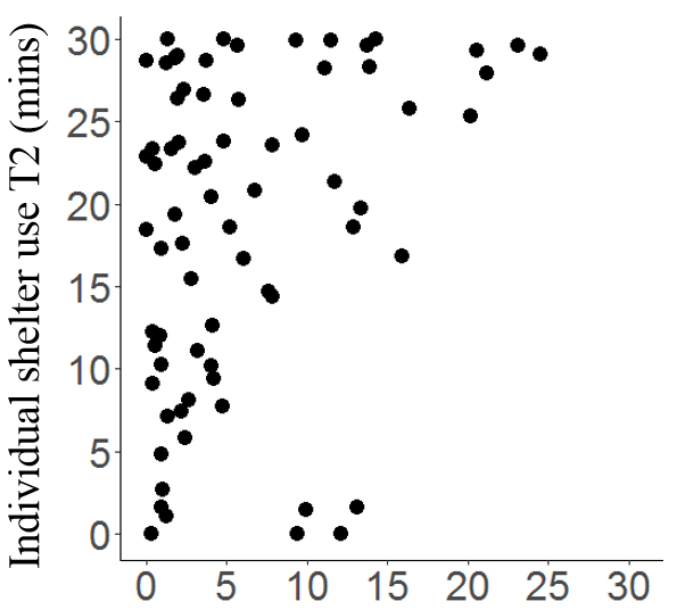

B Individual shelter use T1 (mins)

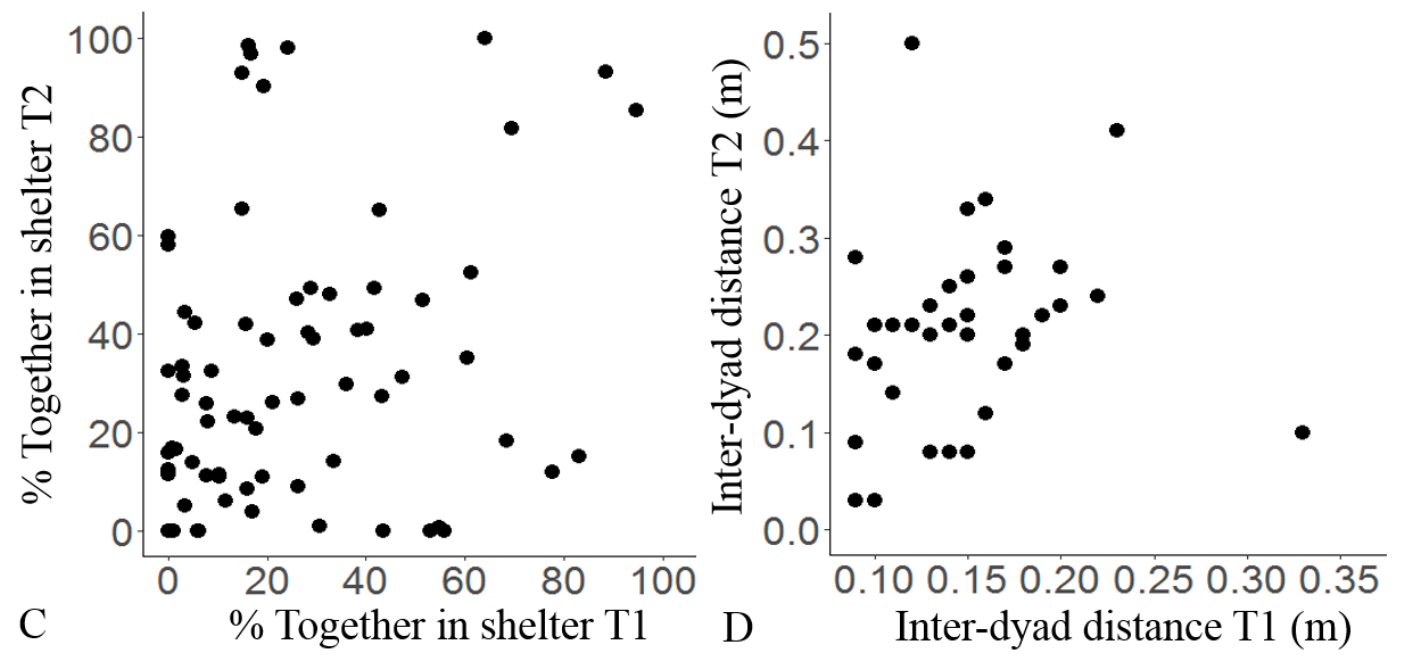

Figure 4.1: Repeatability in (A) total distance travelled (m), (B) individual shelter use (mins), (C) proportion of individual time in shelter with partner (\%) and (D) inter-dyad distance across two trials ( $\mathrm{T} 1$ and $\mathrm{T} 2 ; \mathrm{n}=72$ individuals; $\mathrm{n}=36$ dyads). 


\section{Predictors of behaviour}

Total distance travelled was significantly lower in trial two (estimate \pm s.e. $=-42.34 \pm$ 2.77, $\mathrm{p}<0.001$; Figure 4.2A; Appendix 2; Table 8.6; LMM3). Individual shelter use (estimate \pm s.e. $=12.28 \pm 1.14, \mathrm{p}<0.001$; Figure 4.2B; Appendix 2; Table 8.6; LMM4) and inter-dyad distance (estimate \pm s.e. $=0.06 \pm 0.02, \mathrm{p}=0.002$; Figure 4.2D; Appendix 2; Table 8.6; LMM5) were significantly higher in trial two, indicating that individuals were further apart and hid more in the second compared to the first trial.

Changes in total distance travelled, individual shelter use, and inter-dyad distance were not affected by previous housing or familiarity in the trial but proportion of individual time in shelter with the partner was (Appendix 2; Table 8.6; GLMM2). In unfamiliar dyads the proportion of individual time in shelter with the partner was significantly higher in trial two (estimate \pm s.e. $=1.03 \pm 0.45, \mathrm{p}=0.023$; Figure 4.2C; Table 4.2; GLMM4), but no significant difference was found in familiar dyads (Table 4.2; GLMM3).

A significant negative correlation between total distance travelled and average individual waterborne cortisol concentration was found (estimate \pm s.e. $=-5.65 \pm 2.49$, $\mathrm{p}=0.025$; Figure 4.3; Appendix 2; Table 8.6; LMM3), indicating that individuals with higher average HPI axis activity were less active. Individual shelter use and proportion of individual time in shelter with partner was unrelated to cortisol concentrations (Appendix 2; Table 8.6). 


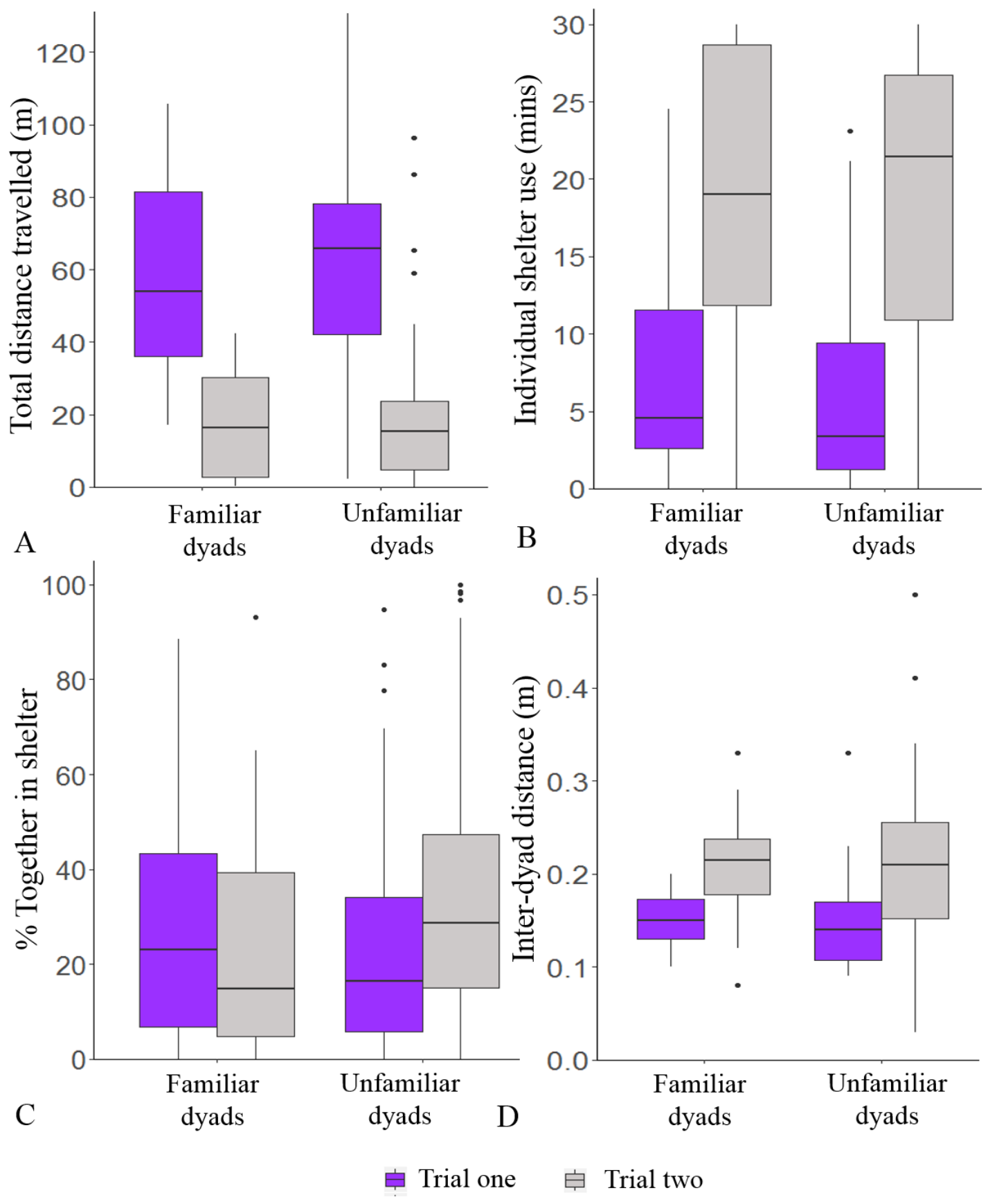

Figure 4.2: Differences in (A) total distance travelled, (B) individual shelter use, (C) proportion of individual time in shelter with partner ( $\mathrm{n}=72$ individuals) and (D) interdyad distance between familiar and unfamiliar stickleback dyads ( $\mathrm{n}=36$ dyads). Box and whisker plots show (for each behaviour measure) average value, first and third quartile values and minimum and maximum values. 
Table 4.2: Post hoc analysis of proportion of individual time in shelter with partner (\%) for each familiarity context; familiar and unfamiliar dyads, $\mathrm{n}=36$ dyads. Statistically significant results are shown in bold.

\begin{tabular}{llcc}
\hline Model & Predictor Variable & Estimate \pm s.e. & p-value \\
\hline Proportion of time in shelter with partner $(\%)$ & & \\
GLMM3 Familiar dyads & & & \\
\% Together in shelter & & & \\
Fixed Effects & Intercept & $-0.02 \pm 0.43$ & \\
& Trial two & $-0.17 \pm 0.58$ & 0.773 \\
& Sex Male & $0.11 \pm 0.71$ & 0.882 \\
Random Effects & & $\chi^{\mathbf{2}}$ & $\begin{array}{c}\text { p-value } \\
\end{array}$ \\
& Fish ID & 53.24 & $<0.001$ \\
& Group ID & 54.82 & $<0.001$ \\
\hline GLMM4 Unfamiliar dyads & & & \\
\%Together in shelter & Intercept & $-0.94 \pm 0.39$ & \\
Fixed Effects & Trial two & $1.03 \pm 0.45$ & $\mathbf{0 . 0 2 3}$ \\
& Sex Male & $1.23 \pm 0.56$ & $\mathbf{0 . 0 2 9}$ \\
& & & \\
Random Effects & Fish ID & 112.53 & $<0.001$ \\
& Group ID & 112.34 & $<\mathbf{0 . 0 0 1}$ \\
\hline
\end{tabular}

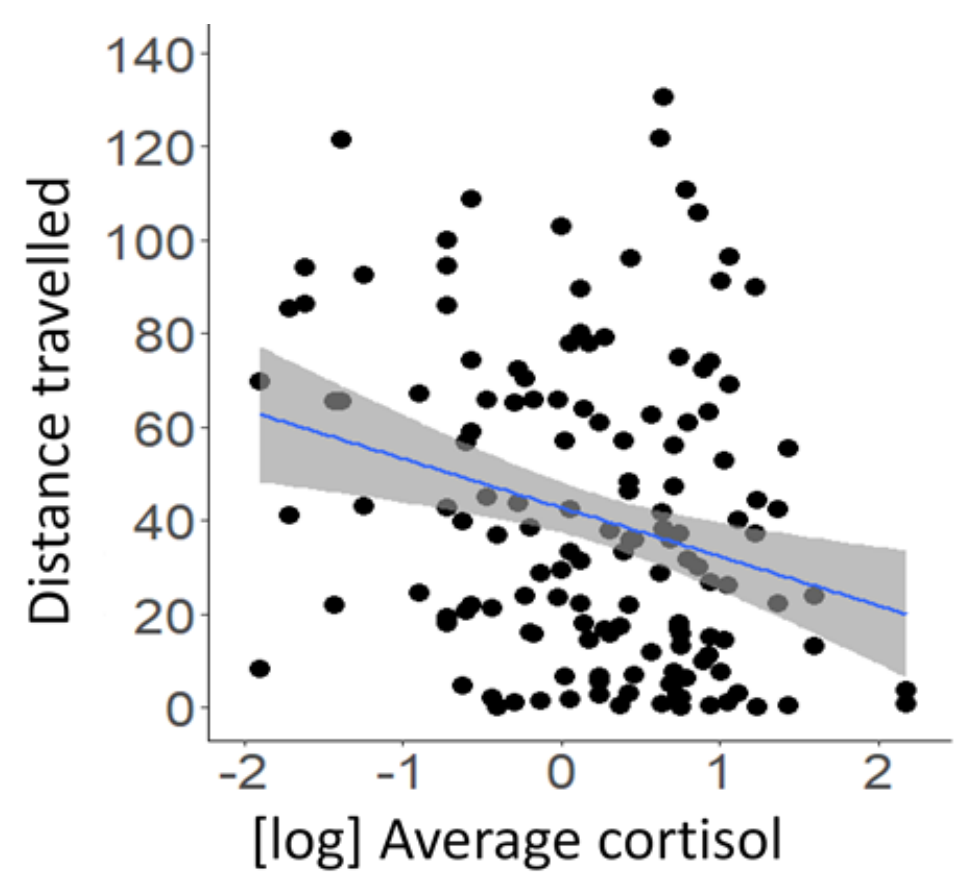

Figure 4.3: Relationship between individuals' average waterborne cortisol (ng/g/h) and total distance travelled $(\mathrm{m}), \mathrm{n}=72$ individuals. The blue line represents the predicted effect of average cortisol and distance travelled, with the shaded area representing the $95 \%$ confidence intervals. 


\section{Discussion}

Recent social context (whether individuals are housed socially or solitary) and the level of familiarity affect behaviour in many animals (De Rouck \& Law 2005; Olsson \& Westlund 2007; Visser et al. 2008; Valero et al. 2009; Dzieweczynski et al. 2012; Meagher et al. 2015; Jolles et al. 2016; Thünken et al. 2016; Keller et al. 2017; LuconXiccato et al. 2017). As most fish species are social with fission-fusion characteristics (i.e. forming large shoals, with individuals regularly joining and leaving), they are a good model to study the effects of recent social context on behavioural responses (Hahlbeck et al. 2004; Huntingford \& Ruiz-Gomez 2009). Previous work on threespined stickleback fish has shown that housing conditions (i.e. housing individuals solitarily, partially solitarily, or socially) can influence repeatability of behaviour (Jolles et al. 2016). Additionally, three-spined stickleback fish stay closer to familiar than unfamiliar conspecifics (Atton et al. 2014) and in several other fish species individuals explore a novel environment more when with familiar individuals (Fathead minnows Pimephales promelas Chivers et al. 1995; cichlid Oreochromis mossambicus Galhardo et al. 2012). However, to date relatively unexplored are possible carry over effects from recent social context to behaviour expressed in a later social context, e.g. whether recent social housing or familiarity affects repeatability and/or behavioural responses when individuals are exposed repeatedly to a novel environment. To address this gap in knowledge, I tested fish dyads with varying recent social context (i.e. housing) and level of familiarity. Furthermore, given that behavioural responses are often coupled with physiological parameters ('coping styles' see Koolhaas et al. 1999 for a review; Øverli et al. 2004; Brelin et al. 2005; Kristiansen \& Fernö 2007; Verbeek et al. 2008; Fürtbauer et al. 2015b), I also tested whether individual HPI axis activity predicted behavioural responses.

\section{Repeatability in behaviour}

Total distance travelled, individual shelter use and proportion of individual time in shelter with partner were repeatable across the two trials and, contrary to my prediction that only solitarily housed but not socially housed individuals would show repeatability in individual behaviours, recent social context had no effect on repeatability. Despite previous research showing solitarily and partially solitarily housed individuals showed stronger repeatability than socially housed individuals 
(Jolles et al. 2016), my results suggest that repeatability in behaviour is not affected by recent context, but perhaps stable to an individual. This could reinforce behaviour as distinct individual personalities that are consistent across time and contexts (Sih 2004; Bell et al. 2009; Stamps \& Groothuis 2010). Previous studies in different species have shown repeatability in activity/distance travelled within individuals, where recent social context was not considered (Gabriel \& Black 2010; Marras et al. 2011; Taylor \& Cooke 2014; Fürtbauer et al. 2015b; Fürtbauer \& Fry 2018).

Additionally, whilst I predicted that the dyadic behaviour (i.e. inter-dyadic distance between fish) would be repeatable in familiar but not unfamiliar dyads, I found this behaviour was not repeatable. One possible explanation for this could be that the current social environment affects an individual's behaviour in a group instead of recent social context. Whilst experience from previous social context can carry over and affect behaviour when tested in isolation (Jolles et al. 2016), this might not be the case for later social behavioural tests. Conformity (i.e. a change in individual behaviour to match partner and/or group behaviour) or social facilitation (the presence of another individual affecting behaviour, which causes certain behaviours to occur more often or perform behaviours they would not perform alone) could explain why the current social environment would be a better predictor of behaviour than recent social context (reviewed by Webster \& Ward 2011). If individuals are changing their behaviour to conform to a partner, this could affect the lack of repeatability as individuals are not consistent in their behaviour responses. For example, if individuals were conforming to social partners we would expect a focal fish's behaviour to change if placed with a different partner, which has been shown in many species (Laland \& Williams 1998; Schuett \& Dall 2009; Kurvers et al. 2013; King et al. 2015; Koski \& Burkart 2015; McDonald et al. 2016; Fürtbauer \& Fry 2018). If social facilitation was occurring, individual's behaviour would be expected to change from partner to partner, which could cause an absence of repeatability. In order to test the effects of conformity and/or social facilitation further, an experiment in which an individual's behaviour is tested first in isolation (i.e. to determine individual behaviour), and then in familiar/unfamiliar social groups after being housed socially/solitarily is required to fully investigate whether behaviour is only carried over into individual isolation behavioural tests. 


\section{Predictors of behaviour}

In addition to repeatability of behaviour, I investigated predictors of behavioural responses including recent housing condition, familiarity with partner, and cortisol measures, and tested for potential changes in behaviour across the two trials. Total distance travelled decreased from trial one to trial two, supporting part of my prediction that individuals would be less active and suggests habituation to the novel environment (Matsunaga \& Watanabe 2010). Habituation to repeated exposure of the same environment has been observed in various species, with individuals showing a decrease in activity levels (Ward et al. 2008; Bolivar 2009; Matsunaga \& Watanabe 2010). However, my prediction that individuals would hide less in the second behaviour trail was not met as individuals increased their time spent in shelter in the second compared to the first trial.

I found no effect of recent housing or familiarity on changes in individual behaviours (distance travelled and individual shelter use) and dyadic behaviour (inter-dyadic distance). Changes in shared shelter use between trials differed between familiarity contexts: unfamiliar dyads spent more time in shelter with a partner in the second trial; whereas no difference was found in familiar dyads. There was no effect of housing on changes in shared shelter use. This supports my prediction that unfamiliar individuals would increase time spent together in the second trial (as a result of familiarity gained in the first trial). Perhaps this result suggests that when individuals are in a novel environment that is not perceived as a stressor (presented in Chapter 3 through the lack of increase in HPI axis activity) and presented with a novel partner, individuals spend more time together due to novelty of partner. Similarly, research in rats has shown that when placed in a neutral cage with either a familiar or unfamiliar individual, it was the unfamiliar individual who the focal individual spent more time interacting (i.e. sniffing) and following (Marco et al. 2011).

Investigations into links between behaviour (total distance travelled, individual shelter use and proportion of time in shelter with partner) and HPI axis activity revealed that individuals' average waterborne cortisol was negatively correlated with activity. This is in line with previous research on copying styles (i.e. suites of correlated physiologcial and behavioual responses in challenging or 'stressful' situations; Koolhaas et al. 1999) in fish, which show that 'reactive' individuals are less active and 
show higher HPI axis activity, whereas proactive individuals are more active and show lower HPI axis activity (Øverli et al. 2004; Brelin et al. 2005; Kristiansen \& Fernö 2007; Verbeek et al. 2008; Fürtbauer et al. 2015b; Castanheira et al. 2017). Given that exposure to the novel environment did not result in increased waterborne cortisol levels (see Chapter 3), this finding suggests that individuals show the characteristics of proactive and reactive coping styles also under 'non-stressful' conditions. Perhaps this suggests that rather than coping styles being a set of physiological and behavioural conditions that enable an individual to cope with an environmental change (Koolhaas et al. 1999; Coppens et al. 2010), 'coping styles' actually describe an individuals' personality. If these 'baseline styles' (i.e. coping styles expressed under non-stressful conditions) are consistent across stressful conditions, then they can be used to predict how individuals will respond to specific environmental changes.

\section{Conclusion}

Overall, this study finds no effect of recent housing condition or familiarity on repeatability and changes in all but one behaviour measure, instead the current social and ecological environment (here NE) appear to explain changes in behaviour. Despite previous research showing that recent social context can have carried over effects onto individual behaviour is isolation (Jolles et al. 2016), there was no carry over effects in this study. I did find repeatability in individual behaviours, which is in line with previous research (Gabriel \& Black 2010; Marras et al. 2011; Taylor \& Cooke 2014; Fürtbauer et al. 2015b; Fürtbauer \& Fry 2018). The unexpected finding that unfamiliar dyads spend more time together in shelter in the second trial two could suggest that when the physical environment is not stressful (i.e. did not cause an increase in HPI axis activity) an individual is interested in their social unfamiliar partner (Marco et al. 2011). Individual cortisol concentration were related to distance travelled, which is in line with previous work on reactive and proactive coping styles (Brelin et al. 2005; Kristiansen \& Fernö 2007; Verbeek et al. 2008; Fürtbauer et al. 2015b; Castanheira et al. 2017), and suggests evidence for these profiles within individuals when not under physiologically stressful conditions. This study suggests that recent social context is less important than the current social and ecological environment in affecting and predicting individuals' behavioural responses. 


\title{
Chapter 5: Repeatability and plasticity in human endocrine traits
}

\begin{abstract}
Human behaviour and personality are influenced by both our external environment, and our own internal physiological processes, including hormones. Different social contexts give us the opportunity to explore how individuals' behavioural and endocrine traits relate to their social experiences, and whether they are repeatable and plastic (i.e. change based on social experience). It is well established that individuals respond differently to social experience due to personality differences and that hormonal differences also occur. However, much of the potential importance of between-individual variation in hormones and personality is ignored because studies are unable to utilise repeated measures of these traits. This study investigates whether individual endocrine traits (cortisol and testosterone) are repeatable before and after people engaged in a social task, and if and how individuals are plastic in their cortisol response. I also explore potential links between cortisol measures and personality traits and perceived stress scores (PSS). I found that cortisol, testosterone and PSS were repeatable before and after a social task indicating consistency in endocrine and behavioural traits. Cortisol showed a non-significant decrease during the social task indicating some level of plasticity. This non-significant decrease in cortisol concentrations was predicted by initial cortisol concentrations, suggesting cortisol plasticity in response to a social task is influenced by individual hormone levels. Additionally, PSS showed a significant decrease during the social task indicating plasticity in PSS. However, PSS and cortisol were not significantly correlated, indicating no relationship between physiological and psychological stress measures. Out of the five Big5 personality traits, only 'conscientiousness' was significantly correlated with mean cortisol concentrations, suggesting a link between physiological stress levels and personality. Individuals are constantly faced with social challenges and I suggest that by highlighting these consistent endocrine profiles and individual differences in plasticity will help researchers to better understand how and why individual differences in endocrine traits and behaviour in response to social experiences persist.
\end{abstract}




\section{Introduction}

Human behaviour and personality are influenced by both our external environment, and our internal physiological processes, including hormones that act as coordinators of social behaviour (Adkins-Regan 2005; Davies et al. 2012). Glucocorticoids (e.g. cortisol) and sex steroids (e.g. testosterone), for example, influence behaviour ranging from parental care (Davies et al. 2012) to leadership (Sherman et al. 2012), and are fundamental to the formation and maintenance of friendships and resulting social networks (Kornienko et al. 2016; Ketay et al. 2017). Even the anticipation of a challenging situation can cause people to experience higher levels of physiological stress, through increased production of cortisol from stimulation of the hypothalamicpituitary-adrenal axis (HPA axis; Kornienko et al. 2014).

Whether an individual experiences higher physiological stress in response to a challenging situation can often be predicted by their behaviour/personality traits (Koolhaas et al. 1999; Conard \& Matthews 2008). Personality traits can influence individuals' behaviour and explain consistencies across situations, and as a result within human behaviour research personality traits are used as another indicator of behaviour (Dall et al. 2004; Nettle \& Penke 2010). The Big 5 dimensions consist of five personality traits (openness, conscientiousness, extraversion, agreeableness and neuroticism) that are stable across time (Goldberg 1990). Each personality trait is associated with different characteristics which describes an individual; openness is associated with experience and unconventional ideas, conscientiousness is associated with organisation and goal-directed behaviour (Goldberg 1990). Extraversion is associated with individuals being active and social, agreeableness is associated with altruistic and cooperative behaviour, whereas neuroticism is associated with negative emotions and anxiety (Goldberg 1990; Cappeliez \& O’Rourke 2002).

Research investigating the links between personality and cortisol has shown that high levels of neuroticism and extraversion are correlated with increased cortisol and that high conscientiousness scores correlated with decreased cortisol under normal conditions (i.e. taking daily measures LeBlanc \& Ducharme 2005; Nater et al. 2010; Hill et al. 2013; Steptoe et al. 2017). Under stressful conditions (e.g. undergoing laboratory stress test) lower openness, lower agreeableness and higher neuroticism has been shown to be associated with lower cortisol (Oswald et al. 2006; Bibbey et al. 
2013). Sex differences in personality-physiology research have also been found; blunted cortisol has been associated with a higher neuroticism score in women, but in men neuroticism and cortisol are positivity correlated (Oswald et al. 2006; DeSoto \& Salinas 2015). Research linking personality and sex steroids are less common and tend to be focused on testosterone, with results showing that higher extraversion and openness are associated with increased testosterone (Smeets-Janssen et al. 2015; Afrisham et al. 2016).

Despite the body of work investigating the relationships between hormones and human personality and/or behaviour, studies tend to link average hormone levels to personality traits (Bateup et al. 2002; DeSoto \& Salinas 2015; Parent-Lamarche \& Marchand 2015) or use single hormone measures (LeBlanc \& Ducharme 2005). Even when multiple samples are taken to investigate hormonal change (Bibbey et al. 2013; Bedgood et al. 2014) or during competition (Edwards et al. 2006), studies do not test for repeatability in endocrine traits (Edwards et al. 2006; Ouyang et al. 2011; Schmidt et al. 2014; reviewed by Taff \& Vitousek 2016). Research examining single or average hormone measure can be extremely insightful (Hamilton et al. 2015) but using a single hormone measure only represents a single time point which may not reliably explain links between hormones and personality as cortisol has a variable diurnal rhythm (Rose et al. 1972; Schoenemann \& Bonier 2018). This means that the potential importance of between-individual variation in hormones and/or behaviour is ignored (Williams 2008; Schoenemann \& Bonier 2018). Understanding the repeatability and plasticity (the expression of different phenotypes in response to environmental change; Taff \& Vitousek 2016) of peoples' hormones and behaviour (and the interactions between them) using a repeated measures design will allow us to better understand and predict individuals' responses to changing environments.

Investigations into the repeatability and plasticity of endocrine traits have become central to the study of non-human animal personality research (see Taff \& Vitousek 2016 for a review of studies on endocrine plasticity) and individuals' ability to cope with change (Stamp et al. 2015). Baseline cortisol measures have been shown to be repeatable in many species, including tree swallows (Tachycineta bicolor Ouyang et al. 2011; Madliger \& Love 2016), great tits (Parus major Ouyang et al. 2011), little penguins (Eudyptula minor Cockrem et al. 2017) and Fijian ground frogs (Platymantis vitianus Narayan et al. 2013). Some recent research has focused on linking hormones 
and behaviour in response to changing environmental conditions. In three-spined stickleback fish (Gasterosteus aculeatus), for example, simulated predator experiments revealed the existence of endocrine personalities which are linked to behavioural differences (Fürtbauer et al. 2015b). Similarly, high cortisol and low exploration behaviour profiles have found in the dark-eyed junco (Junco hyemalis Atwell et al. 2012) and in freshwater fish (Brachyrhophis episcopi Archard et al. 2012).

It is clear from research in non-human animals species that repeatability of endocrine traits needs to be considered also in humans and that the importance of endocrine mechanisms underpinning behaviour cannot be ignored (reviewed by Taff \& Vitousek 2016). However, to my knowledge no studies explicitly and simultaneously investigate individual repeatability and plasticity of endocrine traits in humans. Research investigating changes in hormone concentrations in response to contextual changes such as simulated laboratory stress tests (Kirschbaum et al. 1993; Kudielka et al. 2009) and sports competitions (Bateup et al. 2002), offers insights into human endocrine plasticity. If personality traits (i.e. consistent individual differences in behaviour) are modulated by endocrine traits we would expect consistency in these traits (see Fürtbauer et al. 2015b). Furthermore, we might be able to better understand the potential links between hormones and personality differences in humans if we first access to what extent endocrine traits are repeatable and plastic (reviewed by Taff $\&$ Vitousek 2016).

In this study, I investigated repeatability and plasticity in human endocrine traits (salivary cortisol and testosterone) using a reaction norm approach (Dingemanse et al. 2010). Plasticity in endocrine traits (and perceived stress) were tested in response to changes in peoples' social environment by exposing subjects to a social task. Firstly, I predicted that (i) individuals would exhibit consistency in endocrine traits across the two sampling points (i.e. before and after the social task), based on previous findings in non-human animals ('endocrine personalities' Narayan et al. 2013; Fürtbauer et al. 2015b; Madliger \& Love 2016; reviewed by Taff \& Vitousek 2016; Cockrem et al. 2017).

Secondly, I investigated whether individuals' cortisol and testosterone levels are plastic ('endocrine plasticity' see e.g. Taff \& Vitousek 2016; Gangloff et al. 2017; 
Stedman et al. 2017) in response to the social task, and whether certain individuals are more plastic than others (i.e. exhibit larger changes in hormone concentrations). I predicted that (ii) individuals' cortisol concentrations would be higher before the social task in anticipation of the unknown social task (Bedgood et al. 2014). In addition to cortisol, I also looked at testosterone given that testosterone levels are potentially susceptible to socially induced changes (Eisenegger et al. 2011; Afrisham et al. 2016; Casto \& Edwards 2016; Fürtbauer et al. 2020). For example, in humans testosterone is known to increase significantly during competitive interactions (Edwards \& Kurlander 2010; Casto \& Edwards 2016) and in situations where individuals feel under pressure (Afrisham et al. 2016). However, it has been shown in stickleback fish, testosterone levels were lower due to collection (i.e. when shoaling compared to when alone; Fürtbauer et al. 2020). Therefore, I predicted that (iii) individuals' testosterone concentrations would be higher after the social task due to the pressure of the task. An alternative prediction could be that, in line with previous research, individuals' testosterone would decrease due to collective activity (Fürtbauer et al. 2020)

Thirdly, in addition to endocrine traits, I measured individuals' perceived stress levels because psychological stress can influence physiological stress (Sapolsky 1994), with positive correlations being found between cortisol levels and perceived emotional stress (for a review see Campbell \& Ehlert 2012). I predicted (iv) a positive correlation between individuals physiological and psychological stress. I also predicted and tested for (v) consistency in perceived stress levels across the two sampling points as positive correlations have been shown in validation tests (Cohen et al. 1983). Finally, I also explored the link between cortisol and the Big 5 personality traits.

\section{Methods}

Participants were $n=13$ ( $n=8$ male and $n=5$ female) adults (ages 23-57 years; Table 5.1), voluntarily attending a free art and science games night at Hackney Wick, London ( $5^{\text {th }}$ April 2017). Initially $n=35$ participants signed up to attend the games night but unfortunately, only 13 participants attended on the night. No specific information about the activities was provided prior to the event. On arrival, participants were allocated a participant ID and informed that they would be required to (i) provide two saliva samples, (ii) complete two perceived stress questionnaires, and (iii) one personality questionnaire. Consent was obtained from all participants. Prior to the 
starting the event participants were briefed on the details of the sample procedures (i.e. how to give a saliva sample), the rules of the games and given some background information on the topic of collective behaviour and links to personality. All protocols used in this study were approved by Swansea University's Ethics Committee (Approval Reference CSH-001-2015).

\section{Experiments}

The social task, devised by Heather Barnett (artist in residence with Swansea University at the time of this research project) required participants who were unfamiliar with each other to interact and work together, i.e. they were instructed to adopt behavioural rules of slime mould organisms and work together to achieve cohesion. For this task participants were given three rules (i) no talking, (ii) try to not make eye contact with others (this was to closing mimic slime moulds lack of vision) and to (iii) connect with other participants and collectively move towards the set goal area. There was no scoring or competitive element to this task. The main experiment lasted approximately one hour, and saliva samples were taken before and after the social task (except for participants P11 and P13, see below; Table 1). Following the main experiment, a second activity took place, which involved participants working in smaller groups on a similar task. This task involved each group coming up with three rules for an animal system of their choice. Each group then showed their system to the other groups for them to guess the systems and rules. For participants P11 and P13 saliva samples were taken before and after this second social task.

\section{Saliva sample collection and analysis}

To measure cortisol and testosterone concentrations, saliva samples were collected from all participants (total $n=26$ samples). To assess individual consistency and plasticity in endocrine traits, samples were collected before and after the social task (approximately one hour apart). Participants were instructed not to eat or drink one hour before the event and were asked to indicate any food/drink consumption upon sample collection. This is because acidic or surgery foods can compromise the enzyme immunoassay results (Schwartz et al. 1998; Gordon et al. 2005; Vialard-Miguel et al. 2005; Lovallo et al. 2006; Clements 2013). Approximately $1 \mathrm{~mL}$ of saliva was collected using Salimetrics saliva collection aids (Salimetrics LLC, State College, PA) 
and pre-labelled $2 \mathrm{~mL}$ micro-centrifuge tubes. Saliva samples were frozen after the experiment and were transported to Swansea University on ice. Given the small sample size ( $\mathrm{n}=13)$, I used data collected from an undergraduate practical ( $\mathrm{n}=56$ individuals) to further investigate the potential relationship between psychological and physiological stress levels (Appendix 3; Table 8.8). To assess individual consistency, samples were collected at the beginning and end of a undergraduate practical (approximately one hour apart) and mirroring the techniques used in the main experiment participants were instructed to not eat or drink one hour before the practical (Schwartz et al. 1998; Gordon et al. 2005; Vialard-Miguel et al. 2005; Lovallo et al. 2006; Clements 2013). Approximately $1 \mathrm{~mL}$ of saliva was collected using Salimetrics saliva collection aids (Salimetrics LLC, State College, PA) and pre-labelled $2 \mathrm{~mL}$ micro-centrifuge tubes. Saliva samples were collected at Swansea University which were frozen immediately after collection.

Samples were first thawed and then centrifuged for 3 minutes at a speed of 1,500 rpm to separate cellular material and were then analysed using Salimetrics salivary cortisol and testosterone enzyme immunoassay kits (Salimetrics LLC, State College, PA), following the manufacturer's instructions. Intra-assay coefficients of variation, provided by Salimetrics, were $4.0 \%$ (high) and $7.0 \%$ (low) for cortisol and $2.5 \%$ (high) and $6.7 \%$ (low) for testosterone. Inter-assay coefficients of variation, provided by Salimetrics, were $11.0 \%$ (high) and $3.0 \%$ (low) for cortisol and $14.1 \%$ (high) and $5.6 \%$ (low) for testosterone. Assay sensitivity was $0.007 \mu \mathrm{g} / \mathrm{dL}$.

\section{Questionnaires}

\section{Perceived Stress Scale (PSS)}

In order to assess perceived stress levels, the 10 item PSS (a questionnaire used to assess perceived stress over the last month) was adapted for 'daily use' (Cohen et al. 1983; Appendix 3; Questionnaire 8.1). Two PSS questionnaires were completed by each participant at the same time as saliva samples were collected (i.e. before and after the social task). PSS scores were calculated by reversing the scores for the four positive questions $(0=4,1=3,2=2,3=1$ and $4=0)$ and then summing the scores across all 10 questions, therefore participants could score a total of between 0 and 40 (Cohen et al. 1983). Average PSS scores for each participant are presented in Table 5.1. PSS scores 
for each participant in the additional undergraduate dataset are presented in Appendix 3, Table 8.8 .

\section{'Big 5' Personality questionnaire}

The personality of each individual was assessed using the 50 item International Personality Item Pool (IPIP) questionnaire (Goldberg 1999; Appendix 3; Questionnaire 8.2). This scale analyses the 'Big 5' personality domains; 'openness', 'conscientiousness', 'extraversion', 'agreeableness' and 'neuroticism' (also known as 'emotional stability'). Individuals were given a score of between 10 and 50 for each of the five personality domains. The 'openness' personality domain includes individual traits such as imagination, creativity and abstract thinking, whereas the 'conscientiousness' personality domain includes traits such as organisation, thoughtfulness and ambition (Goldberg 1990). The personality domain 'extraversion' includes individual traits such as sociability, outgoing and assertiveness, 'agreeableness' includes traits such as trustworthy, empathy and affectionate, and the final domain 'neuroticism' includes traits such as anxiety, emotional instability and moodiness (Goldberg 1990).

\section{Statistical analysis}

All statistical analysis was conducted in R (R Development Core Team 2018). Initially, statistical analysis was conducted both with and without participants P11 and P13, who arrived late to identify whether different sampling times affected the results. As there was no significant difference in any of the analyses the results presented include all $\mathrm{n}=13$ participants. To test for individual consistency in cortisol concentrations, testosterone concentrations, and perceived stress levels, Spearman and Pearson correlations were used. To investigate plasticity in endocrine traits and perceived stress, three Linear Mixed Models (LMMs) were used using the packages lme4 and lmerTest (Bates et al. 2014), with cortisol (log-transformed to achieve normal distribution to meet model assumptions), testosterone and perceived stress scores, respectively, fitted as the response variable (LMM1-LMM2; Table 5.2; LMM3; Table 5.3). In each model, context (before and after the social task; categorical effect), age and sex (to control for potential sex and age effects; see Kirschbaum et al. 1992; Harman et al. 2001) were included as fixed effects. Participant ID was included as a 
random effect to account for individual variation in participants' endocrine measures. Model assumptions were checked using graphs of Q-Q plots and standardised residuals vs. fitted values.

To investigate the relationship between initial (before) cortisol concentrations and the change in cortisol concentrations between the two sampling points, a Spearman correlation was used. As this analysis compared individuals with different initial baseline cortisol values, there was potential for regression toward the mean to occur (Barnett 2004; Kelly \& Price 2005). Therefore, I tested for a differential effect using the test for the equality of variances in paired samples (equation 5; Kelly \& Price 2005). Additionally, Spearman correlations were used to investigate the relationship between (i) average PSS and average cortisol concentrations and (ii) average PSS and the percentage change in cortisol between the two sampling points for original and additional data (see methods for details). Initially, a linear mixed model was run with average PSS as the response variable and, average cortisol, change in cortisol and sex as fixed effects. As there were no effects of sex (fixed effect) or individual ID (random effect), I used simple correlations to communicate my findings. Finally, I used Spearman and Pearson correlations to investigate relationships between endocrine measures and the Big 5 personality traits. 
Table 5.1: Summary information for sex, age, salivary cortisol and testosterone concentrations and PSS scores, averaged across two sampling points for $n=13$ male and female participants. PSS is scored on a scale of 0-40, generally a score of 0-13 is considered 'low stress' and a score of 27-40 is considered 'high stress'. Note that a complete table with all measures (pre- and post-hormone concentrations, PSS and scores for each personality trait) for each participant is presented in Appendix 3 Table 8.7).

\begin{tabular}{lllllc}
\hline $\begin{array}{l}\text { Participant } \\
\text { ID }\end{array}$ & Sex & $\begin{array}{l}\text { Age } \\
\text { (years) }\end{array}$ & $\begin{array}{l}\text { Average salivary } \\
\text { cortisol }(\boldsymbol{\mu g} / \mathbf{d L})\end{array}$ & $\begin{array}{l}\text { Average salivary } \\
\text { testosterone }(\mathbf{p g} / \mathbf{m L})\end{array}$ & $\begin{array}{l}\text { Average } \\
\text { PSS }\end{array}$ \\
\hline P1 & Male & 28 & 0.11 & 168.60 & 14.5 \\
P2 & Female & 42 & 0.12 & 63.22 & 16.5 \\
P3 & Male & 35 & 0.12 & 165.45 & 6.5 \\
P4 & Male & 37 & 0.09 & 193.55 & 10 \\
P5 & Male & 57 & 0.15 & 143.54 & 11 \\
P6 & Female & 24 & 0.15 & 111.93 & 14 \\
P7 & Female & 26 & 0.14 & 115.86 & 20.5 \\
P8 & Male & 27 & 0.43 & 98.36 & 4.5 \\
P9 & Male & 25 & 0.16 & 161.73 & 7 \\
P10 & Male & 42 & 0.18 & 200.67 & 16.5 \\
P11 & Female & 54 & 0.16 & 98.33 & 4.5 \\
P12 & Female & 46 & 0.26 & 67.23 & 15.5 \\
P13 & Male & 43 & 0.07 & 122.21 & \\
\hline
\end{tabular}

\section{Results}

\section{Repeatability and plasticity in endocrine traits}

Saliva cortisol concentrations ranged from $0.06 \mu \mathrm{g} / \mathrm{dL}-0.58 \mu \mathrm{g} / \mathrm{dL}$ (median $=0.13$ $\mu \mathrm{g} / \mathrm{dL}, \mathrm{n}=13$ ) and saliva testosterone concentrations ranged from $62.40 \mathrm{pg} / \mathrm{mL}-$ $240.33 \mathrm{pg} / \mathrm{mL}$ (mean $\pm \mathrm{SD}=131.59 \pm 46.41, \mathrm{n}=13$ ). Both cortisol and testosterone concentrations were significantly positively correlated across the two sampling points indicating individual consistency in these endocrine traits (cortisol: Spearman's rho $=0.566, p=0.047, n=13$; Figure 5.1a; testosterone: Pearson's $r=0.792, p=0.001$, $\mathrm{n}=13$; Figure 5.1b). A non-significant trend for cortisol concentrations to be higher before than after the social task was found (LMM1: estimate \pm s.e. $=0.23 \pm 0.11$, $\mathrm{p}=0.068$; Table 5.2; Figure 5.1c), indicating some degree of plasticity in physiological stress levels in response to the social task. There were no effects of age or sex on cortisol concentrations (LMM1; Table 5.2).

Pre-task cortisol concentrations and cortisol change were significantly negatively correlated (Spearman's $\mathrm{rho}=-0.847, \mathrm{p}<0.05, \mathrm{n}=13$; Figure 5.2), indicating that individuals with higher initial cortisol levels showed a greater decrease in cortisol in 
response to the social task. Using the equation described by Kelly \& Price (2005), I tested for a differential effect to test for regression toward the mean as this analysis compared individuals with different initial baseline cortisol values (Barnett 2004; Kelly \& Price 2005). I found evidence for a differential effect $(T=12.07$, df $=13$, $\mathrm{p}<0.05$ ), which suggests that the observed relationship between pre-task cortisol concentrations and cortisol change could be a statistical rather than actual relationship between the variables (Barnett 2004; Kelly \& Price 2005). Therefore, I constructed adjusted values using the equation described by Kelly \& Price (2005) and using these values the significant negative correlation between pre-task cortisol concentrations and cortisol change remained (Spearman's rho=-0.894, $\mathrm{p}<0.05, \mathrm{n}=13$ ). Testosterone concentrations, as expected, were significantly higher in male participants (LMM2: estimate \pm s.e. $=64.97 \pm 18.43, \mathrm{p}=0.005$; Table 5.2) but did not differ significantly between pre- and post-task samples $(\mathrm{LMM} 2$ : estimate \pm s.e. $=5.63 \pm 8.61, \mathrm{p}=0.525$; Table 5.3; Figure 5.1d), indicating a lack of plasticity in testosterone levels in response to the social task. There was no effect of age on testosterone concentrations (LMM2; Table 5.2).

Table 5.2: Factors predicting individual cortisol concentrations ( $\mu \mathrm{g} / \mathrm{dL})$ and testosterone concentrations $(\mathrm{pg} / \mathrm{mL})$ before and after a social task in female and male participants $(n=13)$. Participant ID was included as a random effect. Statistically significant results are shown in bold.

\begin{tabular}{|c|c|c|c|}
\hline Model & Predictor Variable & Estimate \pm s.e. & $p$-value \\
\hline \multicolumn{4}{|l|}{ LMM1: $\log$ (Cortisol) } \\
\hline \multirow{4}{*}{ Fixed Effects } & Intercept & -1.77 & \\
\hline & Context Before & 0.23 & 0.068 \\
\hline & Age & -0.01 & 0.683 \\
\hline & Sex Male & -0.16 & 0.582 \\
\hline \multirow[t]{2}{*}{ Random Effects } & & $\chi^{2}$ & p-value \\
\hline & Participant ID & 7.20 & 0.007 \\
\hline \multicolumn{4}{|l|}{ LMM2: Testosterone } \\
\hline \multirow[t]{4}{*}{ Fixed Effects } & Intercept & 105.65 & \\
\hline & Context Before & $5.63 \pm 8.61$ & 0.525 \\
\hline & Age & $-0.45 \pm 0.82$ & 0.593 \\
\hline & Sex Male & \pm 18.43 & 0.005 \\
\hline \multirow[t]{2}{*}{ Random Effects } & & $\chi^{2}$ & p-value \\
\hline & Participant ID & 5.02 & 0.025 \\
\hline
\end{tabular}



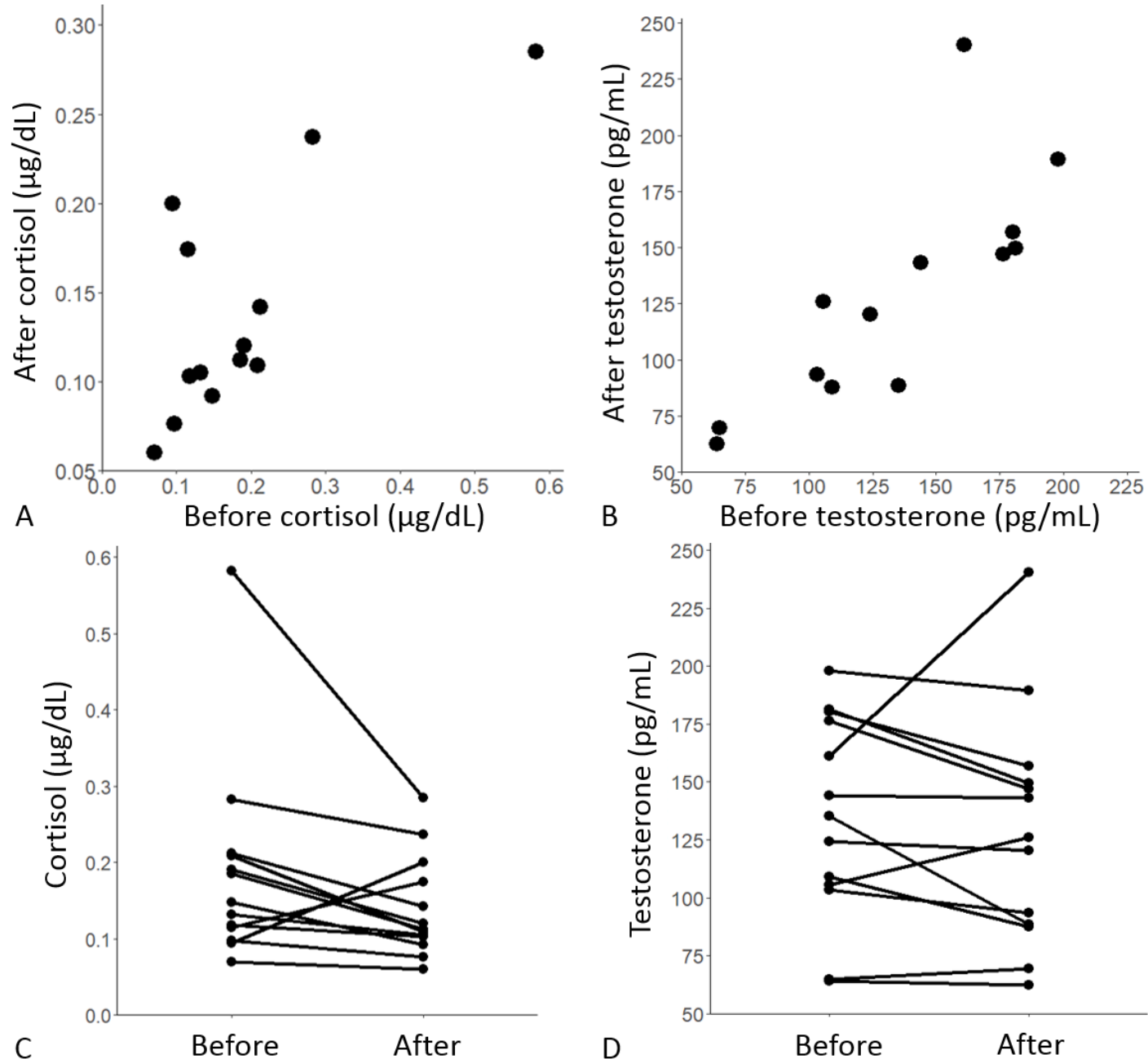

Figure 5.1: (A-B) Repeatability in (A) cortisol and (B) testosterone across two sampling points (before and after a social task; $n=13$ ). (C-D) Reaction norm plots showing change ('plasticity') in (C) cortisol and (D) testosterone concentrations. 


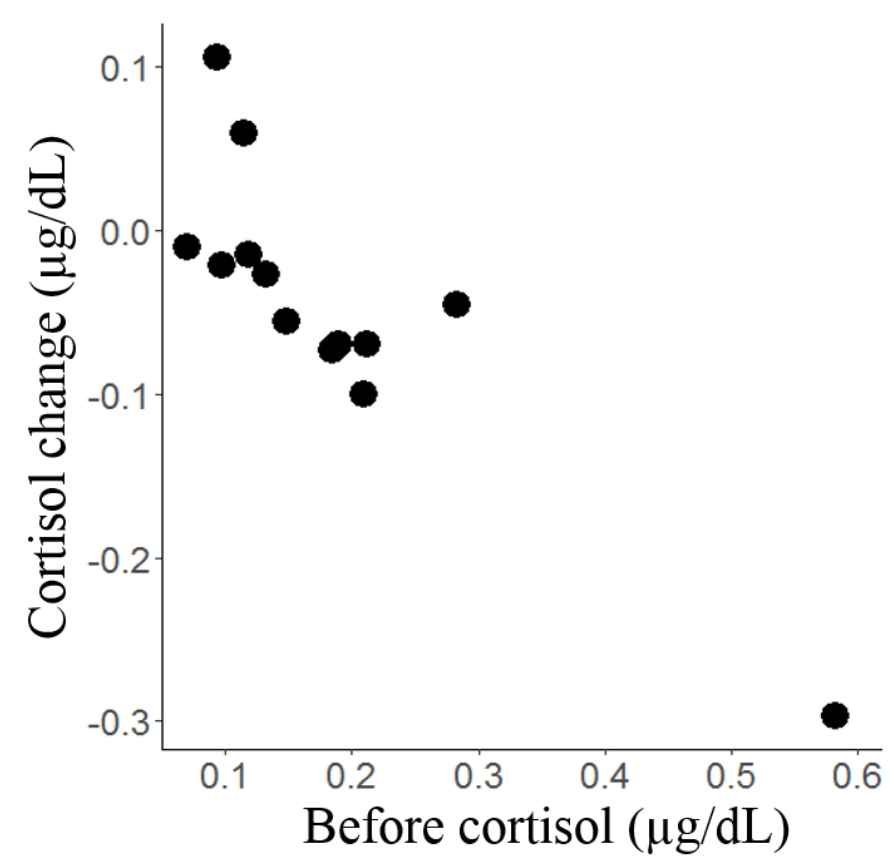

Figure 5.2: Relationship between pre-task cortisol concentrations and the change in cortisol between the two sampling points $(n=13)$.

\section{Repeatability and plasticity in perceived stress}

Perceived stress scores ranged from $3-24$ (mean $\pm \mathrm{SD}=12.15 \pm 5.78, \mathrm{n}=13$ ) and were significantly positively correlated across two sampling points (Pearson's $\mathrm{r}=0.701, \mathrm{p}=0.008, \mathrm{n}=13$; Figure 5.3a), suggesting individual consistency in perceived stress. PSSs were significantly higher before compared to after the social task (LMM3: estimate \pm s.e. $=3.21 \pm 1.36, \mathrm{p}=0.036 ; \mathrm{n}=13$; Table 5.3; Figure 5.3b), suggesting plasticity in perceived stress. Additionally, a non-significant negative correlation was found between PSS and cortisol (LMM3: estimate \pm s.e. $=-18.83 \pm 9.63, p=0.065$, $\mathrm{n}=13$, see Figure 5.4a). Average PSS and the percentage change in cortisol concentrations between the two sampling points were not correlated (Spearman's Rho $=0.234, \mathrm{p}=0.441, \mathrm{n}=13$; Figure 5.4c) and there was no relationship between the percentage change in PSS and the percentage change in cortisol concentrations (Spearman's Rho=0.195, $\mathrm{p}=0.523, \mathrm{n}=13$ ), suggesting that PSS and change in physiological stress levels are unrelated.

Given the small sample size $(n=13)$, I used data available from an undergraduate practical ( $\mathrm{n}=56$ individuals) to further investigate the potential relationship between psychological and physiological stress levels (Appendix 3; Table 8.7). No significant 
relationship was found between PSS and average cortisol (Spearman's Rho $=0.073$, $\mathrm{p}=0.594, \mathrm{n}=56$; Figure $5.4 \mathrm{~b}$ ) or between PSS and percentage change in cortisol concentrations (Spearman's Rho $=0.071, \mathrm{p}=606$, $\mathrm{n}=56$; Figure 5.4d), indicating that PSS and physiological stress levels are unrelated.

Table 5.3: Factors predicting perceived stress in female and male participants $(n=13)$. Participants ID was included as a random effect. Significant results are shown in bold.

\begin{tabular}{llrll}
\hline Model & Predictor Variable & Estimate \pm s.e. & p-value \\
\hline LMM3: PSS & & & & \\
Fixed Effects & Intercept & $20.85 \pm 4.85$ & \\
& Cortisol & $-18.83 \pm 9.63$ & 0.065 \\
& Context Before & $3.21 \pm 1.36$ & $\mathbf{0 . 0 3 6}$ \\
& Age & $-0.13 \pm 0.11$ & 0.255 \\
& Sex Male & -4.22 & \pm 2.39 & 0.113 \\
Random Effects & & & $\boldsymbol{\chi}^{\mathbf{2}}$ & $\boldsymbol{p}$-value \\
& Participant ID & & 2.30 & 0.130 \\
\hline
\end{tabular}

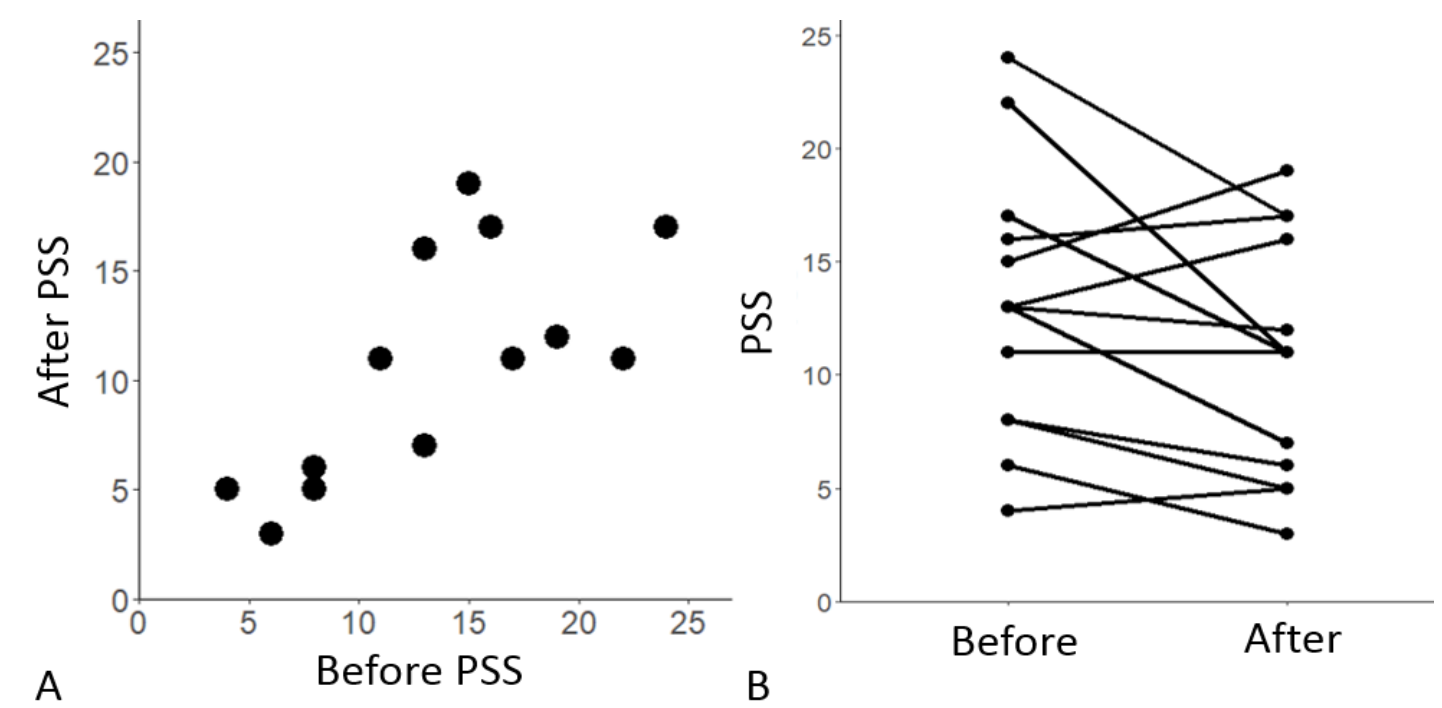

Figure 5.3: (A) Repeatability and (B) plasticity in perceived stress scores (PSS) across two sampling points (before and after a social task, $n=13$ ). 

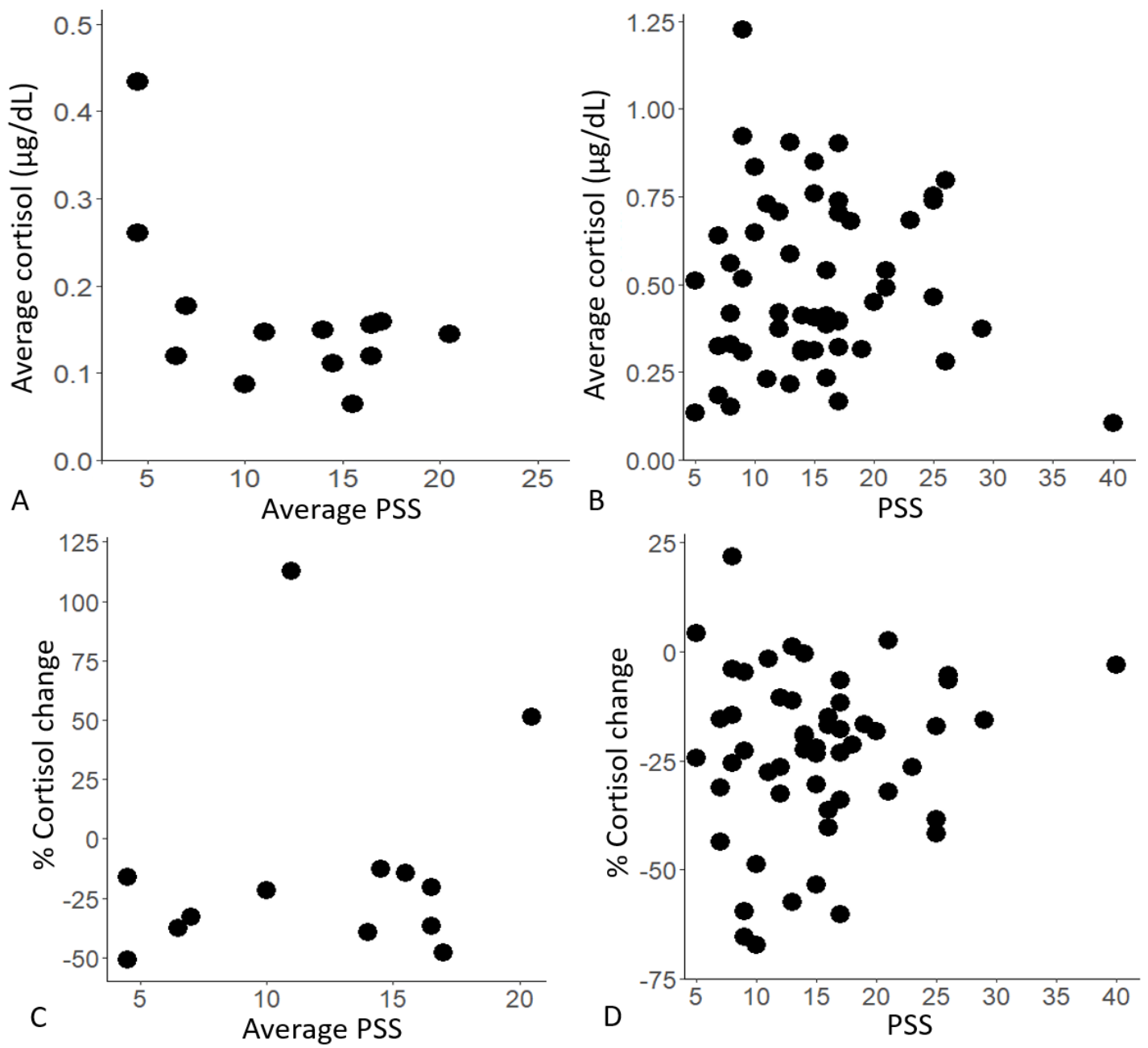

Figure 5.4: Relationship between average PSS and average cortisol in (A) the original data set $(n=13)$ and (B) the additional data set $(n=56)$ and the relationship between average PSS and percentage change in (C) the original data set and (D) the additional data set. 


\section{Links between endocrine traits and personality}

Conscientiousness was significantly positively correlated with average cortisol concentrations (Spearman's Rho $=0.720, \mathrm{p}=0.006, \mathrm{n}=13$; Table 5.4), indicating that individuals with personality characteristics of organisation, thoughtfulness and ambition had higher cortisol concentrations. No other significant relationships were found between the Big 5 personality traits and the average cortisol and testosterone concentrations (Table 5.4).

Table 5.4: Correlations between Big 5 personality traits and average cortisol (Spearman correlations) and testosterone concentrations (Pearson correlations) including p-values in brackets. Statistically significant results are shown in bold.

\begin{tabular}{l|ll} 
& Cortisol & Testosterone \\
\hline Openness & $0.446(\mathrm{p}=0.126)$ & $-0.260(\mathrm{p}=0.390)$ \\
Conscientiousness & $\mathbf{0 . 7 2 0}(\mathbf{p}=\mathbf{0 . 0 0 6})$ & $-0.632(\mathrm{p}=0.687)$ \\
Extraversion & $-0.102(\mathrm{p}=0.739)$ & $0.105(\mathrm{p}=0.734)$ \\
Agreeableness & $-0.025(\mathrm{p}=0.936)$ & $0.001(\mathrm{p}=0.997)$ \\
Neuroticism & $0.319(\mathrm{p}=0.289)$ & $0.193(\mathrm{p}=0.527)$
\end{tabular}

\section{Discussion}

Research into individual differences in human endocrine traits, and how they respond to contextual changes, has increased since the early 2000's but research assessing endocrine consistency and plasticity remains understudied (reviewed by Hau \& Goymann 2015). To explore endocrine plasticity in humans (and any other species), repeated measures are crucial (reviewed by Taff \& Vitousek 2016); however human studies tend to use average (Bateup et al. 2002; DeSoto \& Salinas 2015; ParentLamarche \& Marchand 2015) or single measures (LeBlanc \& Ducharme 2005). The present study investigated individual endocrine consistency and plasticity in response to a social task (i.e. a change in the individuals' social environment) and integrated measures of perceived stress and personality traits. To my knowledge this is the first study to adopt a reaction norm approach in humans, showing consistency and plasticity in endocrine traits. Unfortunately, less people than expected participated in the study and the resulting small sample size leaves some results inconclusive. However, additional data allowed to explore some of the observed trends further. 
Firstly, I found that individuals' cortisol concentrations were significantly correlated across two sampling points, which demonstrates that individuals were repeatable (consistent) in their physiological stress levels. Whilst consistency in glucocorticoids has been found in other vertebrates such as three-spined stickleback fish (Fürtbauer $e t$ al. 2015b), bluegill sunfish (Lepomis macrochirus Cook et al. 2012) and various bird species (see Cockrem 2013a for a review), this, to my knowledge, is the first study that has specifically tested for and reported (short-term) consistency in cortisol concentrations in humans. I also found evidence for repeatability in testosterone concentrations across the two sampling points, which is in line with previous research reporting high correlations of testosterone concentrations in men sampled over multiple occasions (Brown et al. 1978; Liening et al. 2010; Edwards \& Casto 2013). The consistency found in both cortisol and testosterone concentrations supports my first prediction that individuals would exhibit consistency in endocrine traits across the two sampling points.

On an individual level, consistently high cortisol concentrations indicate a higher physiological stress response (Smith \& Vale 2006). Individual differences in cortisol concentrations can occur as a result of the way a stressor is detected and perceived (Adkins-Regan 2005). This could be adaptive when faced with constant environmental changes as an increase in cortisol concentrations increases glucose availability and oxygen intake, this inhibits energetic processes not related to survival (Davies et al. 2012). However, if an individual's cortisol concentration increases in situations where an increased stress response is not needed over long periods it could have a negative effect on health. Chronic elevation of cortisol concentrations has been associated with an increased risk of memory impairment (Oei et al. 2007) and mental health issues (Mondelli et al. 2010).

Consistent individual differences in hormonal traits such as cortisol and testosterone may be linked to behavioural differences. Coping style research (definition: coherent set of behaviour and physiological traits Koolhaas et al. 1999) has shown 'reactive' (behaviourally more flexible and higher HPA axis response Koolhaas et al. 1999; Coppens et al. 2010) and 'proactive' (behaviourally less flexible and lower HPA axis response Koolhaas et al. 1999; Coppens et al. 2010) responses to stressors in rodents (Coppens et al. 2010), fish (Ruiz-Gomez et al. 2011) and birds (Cockrem 2007). My results show that individual endocrine traits are a consistent physiological 
characteristic and perhaps highlights that coping styles could exist in humans, and they may have an important endocrine basis. It could also suggest that human group compositions may benefit from being composed of a mixture of 'proactive' and 'reactive' individuals in order to cope with different challenges. Studies of the effects of behavioural phenotypic composition on the performance of animal groups have found that mixed groups (i.e. groups with more variation in a particular behavioural trait) tend to generally be more successful (Dyer et al. 2009; Modlmeier et al. 2012). For example, mixed groups of bold and shy guppies (Poecilia reticulata) have a higher group foraging success (Dyer et al. 2009) and ant (Temnothorax longispinosus) colonies with more behavioural variation are more productive (Modlmeier et al. 2012).

As personality traits are used as a proxy of behaviour in humans, I investigated the potential relationships between the Big 5 personality traits and average cortisol and testosterone concentrations, respectively. One of the characteristics associated with the personality trait conscientiousness is an increased awareness and vigilance (Rose et al. 2002), which could suggest that individuals scoring high in this trait could respond quicker to changes in the environment; making them behaviourally more flexible. This is in line with my results that found that individuals scoring high for conscientiousness have higher HPA axis activity (i.e. increased cortisol), further suggesting that in humans coping styles involve personality traits and endocrine concentrations.

Secondly, I explored plasticity in cortisol levels and found a trend towards cortisol concentrations being higher before than after the social task. Although not significant, this finding is in line with my second prediction that individual's cortisol concentration would be higher before the social task and suggests a certain degree of cortisol plasticity. This is in line with previous research showing that cortisol was higher before individuals took part in a TSST (Trier Social Stress Test) stress test (Khalfa et al. 2003) or a competitive sports match (Urhausen et al. 1987), perhaps indicating that cortisol increases in anticipation of a task. Cortisol is released in response to a stressful stimulus (a 'stressor') to increase blood flow and make an individual more alert, when the stressor is removed from the environment cortisol is downregulated (Davies et al. 2012). On an ultimate level it could be advantageous for individuals to be plastic in their cortisol responses; especially in dynamic environments (reviewed by Taff $\&$ Vitousek 2016). If individuals are able to increase cortisol production during a challenging situation, they would benefit from physiological responses that could help 
overcome the challenge (e.g. increase blood flow) and decreasing cortisol production when the stressor is removed would ensure negative consequences of constant cortisol stimulation (i.e. chronic stress Smith \& Vale 2006). Testosterone concentration, in contrast, did not change in response to the social task, failing to support my third prediction that individual's testosterone would change after the social task. This result may be explained by the absence of a competitive component/context for which changes in testosterone concentrations are often reported (Trumble et al. 2012; Casto et al. 2014; Casto \& Edwards 2016), suggesting that testosterone plasticity may only be found in response to competition/challenging situations.

I further explored whether certain individuals are more or less plastic by investigating whether individual's initial cortisol levels were related to changes in cortisol concentrations. I found that individuals with higher initial cortisol concentrations showed a stronger decrease therein (i.e. greater plasticity). These results perhaps suggest that individuals that showed a greater plastic response are more reactive individuals and are individuals that show more flexible slopes in their response to the environmental change (see Taff \& Vitousek 2016 for a review on endocrine flexibility). In my study, individuals' cortisol concentrations were lower after compared to before the social task, indicating that perhaps individuals showed increased cortisol concentrations in anticipation of the unknown social task. This suggests that individuals were more reactive and able to decrease their cortisol in response to being in a stressful environment. If individuals with higher baseline concentrations are more reactive, it could be expected that these individuals (the ones that in this study showed a stronger decrease in cortisol) to show a stronger increase in cortisol concentrations in response to stressful environments; an area for future endocrine flexibility research. This is advantageous for individuals in dynamic environments subject to change as individuals can quickly alter their cortisol concentrations when needed (Dall et al. 2004; Canale \& Henry 2010). This fits in with the theoretical arguments that individuals with more flexible slopes (i.e. greater flexibility in reaction norms) are better able to deal with a dynamic environment (reviewed by Taff \& Vitousek 2016). Alternatively, it could be that individuals with higher baseline cortisol concentrations are operating at their maximum reactive scope, and any further increase would push individuals into homeostatic overload (Romero et al. 2009). It would be interesting for future to explore individual endocrine 
flexibility in a range of environmental conditions. Whilst this study shows that interindividual differences in initial cortisol concentrations can predict the level of plasticity and highlights some insights to individual reactivity, we did not assess repeatability in plasticity. Future studies should investigate whether individual plasticity is consistent across a variety of contexts (Schoenemann \& Bonier 2018).

The investigation into a link between psychological and physiological stress revealed a non-significant negative correlation between perceived stress scores and cortisol concentrations, which provides some support for my fourth prediction that there would be a positive correlation between individuals' physiological and psychological stress measures. Whilst other studies have also failed to find a relationship (Vedhara et al. 2003; Gidlow et al. 2016; Olstad et al. 2016) there have been studies that find a positive correlation (Kalra et al. 2007; Karlén et al. 2011; Donker 2015; Sladek et al. 2016). The discrepancy in these findings could be due to the environments/contexts in which stress was measured, and perhaps physiological and psychological stress are correlated only in stressful environments. Due to the relatively small sample size in my study, additional data was collected and analysed and showed no relationship between perceived and physiological stress. Despite the absence of a link to physiological stress levels, perceived stress scores were repeatable, indicating consistency in participants' self-assessment of perceived stress and supporting my fifth prediction that perceived stress levels would be consistent across the two sampling points. Similar to cortisol concentrations, I also found a trend towards PSS being higher before the social task, which could reflect individual's anticipation of the unknown and possibly a stressful/challenging situation. In this study I show that individual's perceived stress can be used to accurately represent their psychological stress levels, and whilst we found no relationship between psychological and physiological stress measures perhaps perceived stress does link to physiological stress under certain contexts (i.e. stressful environments).

\section{Conclusion}

Overall, and despite the small sample size, this study demonstrates that both cortisol and testosterone concentrations are repeatable over a short time period. Such consistent individual differences in endocrine traits may underlie consistent behavioural/personality differences which can be important for group functioning and 
performance (Dyer et al. 2009; Modlmeier et al. 2012). It also shows some evidence of cortisol plasticity, which suggests that HPA axis activity was increased in anticipation of the task. Hence, studying individual differences in endocrine traits may help broaden our mechanistic understanding of behavioural/personality variation (and maintenance) also in humans. The results suggest that individual differences in cortisol responses are linked also to the degree of plasticity therein, suggesting that endocrine personalities may also affect the ability to cope with dynamic environments, with some individuals being more flexible than others (Taff \& Vitousek 2016). 


\title{
Chapter 6: Repeatability and plasticity in sheep wool cortisol in response to changing food availability
}

The work presented in this chapter formed part of a published research article and conference poster presentation (Appendix 5):

Fürtbauer I., Solman C., \& Fry A. (2019) Sheep wool cortisol as a retrospective measure of long-term HPA-axis activity and links to body mass. Domestic Animal Endocrinology 68, 39-46

Fürtbauer, I., Solman, C., \& Fry, A. (2018) Sheep wool cortisol: Intra-individual repeatability and plasticity in response to grazing related changes in body mass. 9th European Conference in Behavioural Biology (ASAB), Liverpool, UK.

\begin{abstract}
Understanding individual's phenotypic plasticity (both physiologically and physically) is important to fully investigate and understand how animals respond to environmental changes. The hypothalamic-pituitary-adrenal (HPA) axis activity can provide insight to an individual's physiological activity and using chronic (long-term) measures of HPA activity, allows for assessment of phenotypic plasticity in response to gradual environmental changes, such as variations in food availability. Whilst research into the relationship between short-term HPA axis activity and food availability has been demonstrated in cortisol concentrations when food is limited (i.e. restricted in experiments), little is known about changes in chronic HPA axis activity in response to changing food availability. Here, I use wool cortisol analysis to assess chronic HPA axis activity of $n=33$ Welsh mountain sheep that experienced changes in food availability as a result of two one-month long (high-food quality) grazing periods. Wool samples and body mass measurements were taken from individuals before and after grazing. Body mass increased during both grazing periods. Wool cortisol concentrations (WCC) were found to be repeatable and significantly higher before grazing compared to after grazing. WCC was also significantly negatively correlated with body mass, indicating that heavier individuals exhibited lower WCC. Additionally, cortisol plasticity (measured as the percentage change in WCC across samples) was predicted by individuals' initial WCC, with individuals with higher WCC before grazing showing a larger decrease in WCC after grazing. Taken together these results suggest that WCC (a long-term measure of HPA activity) can be used as an indicator of sheep nutritional status and show that endocrine plasticity can be dependent on the environmental conditions an individual experiences. Cortisol plasticity was predicted by pre-grazing WCC, which could suggest these individuals are more reactive to environmental change, in this case changing available nutritional context.
\end{abstract}




\section{Introduction}

During an acute (short-term) stress response, an animal perceives a stressor and the hypothalamic-pituitary-adrenal (HPA) axis is activated, which causes a cascade of physiological and behavioural responses that enables an individual to cope with the stressor and return to homeostasis (Nelson 2011). However, if the HPA axis is repeatedly activated over a long period of time, and does not return to homeostasis, this results in a chronic stress response (Nelson 2011). Chronic stress, i.e. long-term activation of the HPA axis, can have numerous negative consequences to an animal's health and fitness, including, for example, reduced growth and reproduction (Caslini et al. 2016). Taking biological measurements that represent an animal's physiological stress response over long-term can enable researches to gain a deeper understanding of potential chronic (long-term) activation of the HPA axis (Burnard et al. 2017; Heimbürge et al. 2018). Long-term measures of HPA axis activity can therefore provide a window for viewing if and how individuals' physiology responds to changes in their environment (i.e. their plasticity), which can be vital for their survival (Ghalambor et al. 2007). Cortisol plasticity (i.e. changes in cortisol levels across time and/or contexts), in particular, can be used to determine how changes in the environment affect an individual's physiological stress response (Wingfield 2013).

For many species, the effects of environmental changes are not instant but occur over a period of time and there can be a time lag between the change and the animal responding (Gabriel et al. 2005). Therefore, measuring short-term physiological stress responses (which are subject to daily fluctuations) will not give an accurate representation of how an individual is responding to longer-term changes (Russell et al. 2012; Caslini et al. 2016). Measures of chronic or long-term physiological stress responses are thus needed to assess HPA axis activity in response to a gradual environmental change (Burnard et al. 2017). For example, hair cortisol concentrations represent long-term cortisol secretion, as shown in dairy cows (Bos taurus, Comin et al. 2011; Moya et al. 2013; Peric et al. 2017), pigs (Sus scrofa domesticus, Casal et al. 2017), red deer (Cervus elaphus Caslini et al. 2016), domestic cats and dogs (Felis catus and Canis lupus familiaris, Accorsi et al. 2008), rhesus macaques (Macaca mulatta, Davenport et al. 2006), vervet monkeys (Chlorocebus pygerythrus, Fairbanks et al. 2011), and humans (Homo sapiens, D’Anna-Hernandez et al. 2011; van Holland et al. 2012). 
Measuring hair cortisol concentrations has its advantages because (i) hair samples are relatively non-invasive to collect, (ii) are not subject to daily fluctuations (and therefore represent an average cortisol measure over a longer period), (iii) can be used as a retrospective calendar, (iv) and can be easily stored at room temperature (Russell et al. 2012; Stalder \& Kirschbaum 2012; Burnard et al. 2017). Hair samples are therefore a good candidate for taking repeated measures of chronic HPA axis activation, providing an indication of individuals' physiological stress response over an longer time period (Russell et al. 2012). However, repeated sampling requires capture of the same individual through time, and where individuals range over large areas and identification can be difficult (Burnard et al. 2017), this can become challenging. Additionally, the 're-shave' approach (which enables researchers to investigate how specific changes influence chronic stress physiology, by knowing that new hair was grown during the experimental period) requires that hair samples are taken from the same location, which can be difficult to (re)locate (Comin et al. 2011; Burnard et al. 2017).

Agricultural settings and farm livestock provide researchers with greater opportunity to investigate chronic physiological stress in multiple individuals, and in response to natural environmental changes (Burnard et al. 2017). For example, previous research in dairy cows using hair cortisol measures found that cortisol increased during periods of moving cows from indoor winter to summer grazing, which was suggested to be the result of the change in physical environment and diet (Comin et al. 2011). Similarly, Peric et al., (2017) explored changes in hair cortisol levels in cows moving to high mountain pastures and compared to cows remaining in indoor management. Results showed that hair cortisol increased during the moving period for individuals moving to high mountain pastures (as a result of a change in the physical environment) but remained constant in individuals kept indoors (those not experiencing a change in the physical environment; Peric et al. 2017).

In addition to cattle experiencing changes in stress physiology as a result of seasonal moving (Comin et al. 2011; Peric et al. 2017), changes in food availability are also likely to cause a stress response. Food availability alters with environmental variation within and across seasons or years, and periods of high or low food availability can affect an individual's physical condition (Kruuk \& Parish 1985; Strum 1991; Auer et al. 2015). In particular, individuals' body mass (and changes therein) can give an 
overall indication of physical well-being (Hicks et al. 1998; Sánchez-Muros et al. 2013), with positive correlations between food availability and body mass found in e.g. cattle (Monteiro 1975; Bines \& Morant 1983; Taylor et al. 1986; Basarab et al. 2003; Dillon et al. 2003) and sheep (Ovis aries Blaxter et al. 1961; Thornton et al. 1979; Abouheif et al. 2013; Swelum et al. 2017). In addition to food availability being a proxy of physical well-being, it has also been shown to be an indicator of physiological state (Kitaysky et al. 1999; Auer et al. 2015). Cortisol is a product of the HPA axis produced when individuals are under stress, it results in a cascade of responses that enable individuals to cope with the stressor and ultimately return the individual to homeostasis (Nelson 2011). In animals, a key environmental stress is food availability (i.e. whether individuals can obtain enough food daily), especially because food availability can vary seasonally and is dependent on other factors e.g. population density (Nelson 2011). If an individual is unable to take in enough nutrients this can put the body under significant stress, as individuals will not have enough nutrients required for metabolic processes (Nelson 2011). This results in the body breaking down its fat stores to use as energy for survival as a result of redistribution of body fat - if this continues over a prolonged period an individual's body mass can decrease (Manenschijn et al. 2011a; Nelson 2011). Using food manipulation studies, research has shown negative correlations between cortisol concentrations and food availability/abundance in red colobus monkeys (Procolobus rufomitratus, Chapman et al. 2015), ring-tailed lemurs (Lemur catta, Fardi et al. 2018), Guatemalan black howler monkeys (Alouatta pigra, Behie et al. 2010), black-legged kittiwakes (Rissa tridactyla, Wingfield \& Kitaysky 2002), song birds (Melospiza melodia, Clinchy et al. 2004), American white ibis (Eudocimus albus, Herring et al. 2011), common murres (Uria aalge, Kitaysky et al. 2007) and rainbow trout (Oncorhynchus mykiss, Blom et al. 2000). Additionally, a decrease in cortisol levels along with an increase in weight has been found in animals such as rodents (Cavia aperea, Schumann et al. 2014), pigs (Sus scrofa, Trevisan et al. 2017), polar bears (Ursus maritimus, Macbeth et al. 2012) and pied tamarins (Saguinus bicolor, Price et al. 2019).

Whilst research has started to explore the links between food availability, endocrine responses and morphological condition, previous research has focused on artificially manipulating food availability in the laboratory (Fokidis et al. 2012). Additionally, cortisol responses to changes in food availability tend to use cortisol measures that 
represent relatively short periods of time such as cortisol in blood (Blom et al. 2000; Kitaysky et al. 2007) and faecal samples (Chapman et al. 2015). Therefore, whilst these laboratory studies using short-term measures of cortisol levels offer insight into the relationship between cortisol and food availability, the potential effects of changing food availability upon long-term HPA-axis activity in the wild have not yet been investigated. Using measures of chronic (long-term) physiological stress such as hair/wool will enable researchers to assess links between physiology and physical condition in response to changes in food availability and associated changes in body mass. Understanding how individual and group physiological responses and physical condition change with food availability will help to better understand whether environmental change affects groups and individuals differently. This may help us predict responses to other environmental changes (e.g. habitat destruction/fragmentation) by highlighting whether changes need to be considered on an individual and/or group level, a key challenge facing animals in natural environments (Stubsjøen et al. 2015).

In this study, I investigate long-term repeatability and plasticity in wool cortisol concentrations (WCC) in Welsh mountain sheep (Ovis aries) that are moved back and forth to a high-quality upland pasture where they were allowed to freely graze. Wool fibre growth is almost continuous, which means it can be used as an indicator of an individual's cortisol timeline in the same way in which hair cortisol concentrations can be used a retrospective calendar (Caslini et al. 2016; Burnard et al. 2017). Research has successfully used wool samples to assess chronic wool cortisol concentrations in sheep in response to water restriction (Ghassemi Nejad et al. 2014), indicating that wool, like hair, can also be used to assess chronic HPA axis activity. I analysed WCC using a reaction norm approach, which describes individual flexibility by analysing the elevation (individual WCC) and the slope (the measure of plasticity) for each individual's response to grazing (David et al. 2004; Dingemanse et al. 2010). To my knowledge, this is the first study to adopt this approach using measures of long-term physiological stress. Wool samples were taken, and body mass recorded from individuals before grazing and after grazing (total of four samples per sheep) allowing me to investigate repeatability and plasticity in sheep wool cortisol in response to changing food availability experienced with the changing grazing regime. 
I hypothesised that changes to food availability will directly influence sheep body condition and thus affect their physiological stress response, and that individuals should consistently differ in this response (Fokidis et al. 2012; Abouheif et al. 2013; Swelum et al. 2017). First, I expected sheep to show increased body mass after each grazing period (prediction 1), since changes in body mass can be used as a proxy for changes in food availability (Kruuk \& Parish 1985; Strum 1991; Yom-Tov \& Geffen 2011; Sánchez-Muros et al. 2013). Second, because cortisol is known to increase when food resources are low (Kitaysky et al. 2007; Herring et al. 2011; Fokidis et al. 2012), I predicted sheep would show a decrease in their WCC after grazing (prediction 2). Given the expected relationship between body condition and WCC, at an individual level, I expected individuals with heavier body mass would have lower WCC (prediction 3; Herring et al. 2011; Fokidis et al. 2012). Finally, I predicted consistent individual differences in WCC (prediction 4; Cockrem 2013b; Narayan et al. 2013; Fürtbauer et al. 2015b; Madliger \& Love 2016), based on the growing evidence for such differences (Narayan et al. 2013; Fürtbauer et al. 2015b; Madliger \& Love 2016; Cockrem et al. 2017).

\section{Materials and methods}

$\mathrm{N}=33$ Welsh mountain barren ewes (Ovis aries), which formed part of a larger project investigating sheep utilisation of resources in an uplands landscape (Lush et al. 2018; http://uplands-n2o.bangor.ac.uk/), were studied. The work and methods used were approved by Swansea University's Animal Welfare and Ethical Review Group (References IP-1516-5) and by Bangor University's College of Natural Sciences Ethics Committee (Ethics approval code CND2016DC01). This study included two 1month long grazing periods, where sheep were allowed to graze freely in a semiimproved (high-quality) enclosed 11.5 ha upland pasture at Bangor University's Henfaes Research Centre, Abergwyngregyn, North Wales (see Figure 1 for details). Prior to and in between experimental grazing periods sheep grazed on unimproved grassland. These grazing periods allowed for repeated wool sample collection; $n=4$ samples for $n=27$ and $n=2$ samples for $n=6$ individuals (note that $n=3$ sheep were replaced for the second grazing period due to health reasons). Sheep were weighed before and after grazing in the uplands pasture (mean body mass across all 
measurements $\pm \mathrm{SD}=38.77 \mathrm{~kg} \pm 6.55 \mathrm{~kg} ; \mathrm{n}=33$ sheep). The age of each individual was recorded and for statistical analysis individuals were placed in two age classes, 3-4 $(n=18)$ and 5-6 year $(n=15)$ olds.

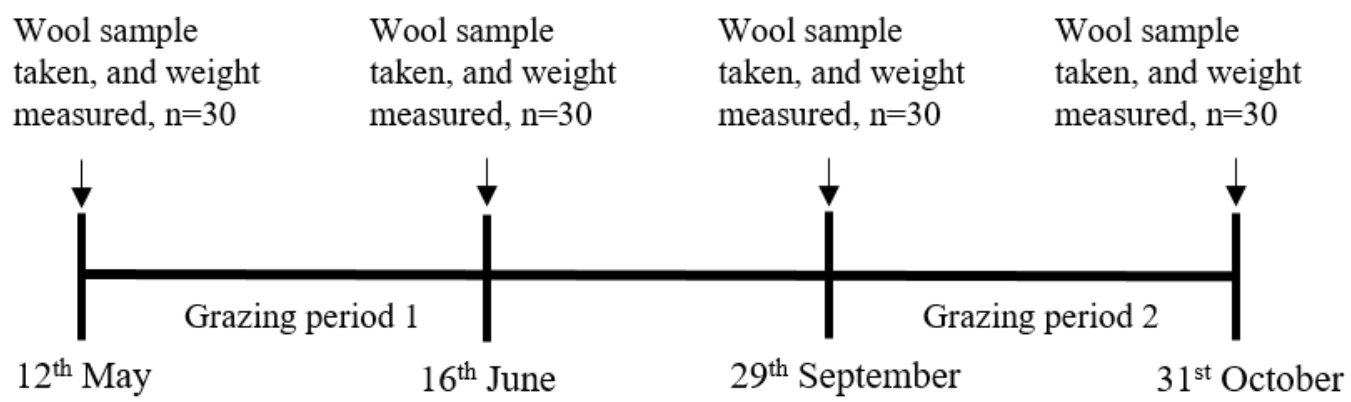

Figure 6.1: Experimental timeline showing the two grazing periods. At the start of each grazing period, a wool sample was taken, and body mass was measured. Sheep were then allowed to graze freely in an upland pasture. At the end of the grazing period another wool sample and body mass measure were obtained. The second grazing period occurred three months later, and the sampling procedure mirrored the first grazing period.

\section{Wool sample collection and storage}

Wool samples (total $n=120 ; n=4$ samples for $n=27$ individuals and $n=2$ samples for $\mathrm{n}=6$ individuals; see sampling schedule above; Figure 6.1) were collected by I. Fürtbauer from the lower back of each sheep using commercially available pet clippers, which were cleaned in-between each individual to avoid contamination (Davenport et al. 2006; Ghassemi Nejad et al. 2014). During both grazing periods, the second wool sample was taken from the same area as the first to ensure the cortisol measured in the second sample reflected the grazing period (shave re-shave approach; Accorsi et al. 2008; Comin et al. 2011). The wool samples were stored in aluminium foil within labelled paper envelopes at room temperature until extraction and analysis (e.g. Davenport et al. 2006; Ghassemi Nejad et al. 2014; Stubsjøen et al. 2015; Caslini et al. 2016).

\section{Extraction and measurement of wool cortisol}

Wool samples were processed following published procedures (e.g. Davenport et al. 2006; Ghassemi Nejad et al. 2014). In brief, approximately $250 \mathrm{mg}$ of each wool sample was washed twice with $5 \mathrm{~mL}$ isopropanol. Isopropanol was removed and samples were then placed in individual aluminium weighing boat and allowed to dry 
at room temperature for 7 days under a protected hood (Paulsen et al. 2001). Following drying, approximately $50 \mathrm{mg}$ of wool was placed in $2 \mathrm{~mL}$ microcentrifuge tubes, finely cut using scissors, and weighed (dry weight mean $\pm \mathrm{SD}=0.053 \pm 0.002, \mathrm{n}=120$ ). Where wool samples were long in length $(n=20)$, a subsample of $2.5 \mathrm{~cm}$ was taken from the end proximal to the skin. A subsample was taken to ensure that the wool used for cortisol analysis came from the first $2.5 \mathrm{~cm}$ of wool in all samples. This was done to ensure comparison of cortisol from the same time frame (i.e. cortisol analysed from growth during the experimental month would match the same period of time before the experimental month). Subsequently, $1 \mathrm{~mL}$ of methanol was added to each microcentrifuge tube. Tubes were vortexed for 10 minutes and incubated for 24 hours at room temperature to extract steroid hormones.

Following the 24-hour extraction period, samples were vortexed for 10 minutes and centrifuged (using a VWR micro star 17/17R centrifuge) for 5 minutes on the highest speed $(13,330 \mathrm{rpm})$. Then $600 \mu \mathrm{L}$ of the steroid-containing supernatant was transferred to a glass tube and completely dried under a stream of nitrogen gas at $38{ }^{\circ} \mathrm{C}$. Once dried, samples were re-suspended in $400 \mu \mathrm{L}$ of the assay diluent (containing phosphate buffer) provided in the immunoassay kit (Ghassemi Nejad et al. 2014). Samples were analysed for cortisol concentrations using a commercially available saliva enzyme immunoassay (Salimetrics LLC, State College, PA). This kit has been used to analyse hair cortisol concentrations in sheep (Ghassemi Nejad et al. 2014) and numerous other species including humans (Hodes et al. 2017; Hoffman et al. 2017; Tarullo et al. 2017; Ursache et al. 2017), non-human primates such as chimpanzees (Pan troglodytes, Yamanashi et al. 2018), rhesus macaques (Macaca mulatta, Hamel et al. 2011; Dettmer et al. 2017; Wooddell et al. 2017), pig-tailed macaques (Macaca nemstrina, Grant et al. 2017), ring-tailed lemurs (Lemur catta, Fardi et al. 2018) and other mammals such as brown bears and polar bears (Ursus arctos, Ursus marittimus, Kroshko et al. 2017), brown hears (Lepus europaeus, Esposito et al. 2017), domestic dogs (Canis lupus familiaris, Grigg et al. 2017b), cattle (Bos taurus, Ghassemi Nejad et al. 2017; Lockwood et al. 2017) and pigs (Sus scrofa, Casal et al. 2017; van der Staay et al. 2017). Samples from the same individual were run on the sample plate in duplicate. The sensitivity of the assay was $0.007 \mu \mathrm{g} / \mathrm{dL}$. The intra-assay coefficients of variation were $3.0 \%$ for high and $9.0 \%$ for low quality controls, respectively. Interassay coefficients of variation were $4.0 \%$ for high and $8.0 \%$ for low quality controls, 
respectively, which were run in quadruplicate on each plate ( $n=4$ plates). Cortisol concentrations are expressed as pg/mg wool.

\section{Statistical analysis}

Linear mixed models (LMMs) were performed in R (R Development Core Team 2018) using the packages lme4 and lmerTest (Bates et al. 2014). In all models, WCC was log transformed to meet the model assumptions of linear mixed models (LMMs; so that cortisol concentration fits normal distribution; Bates et al. 2014). LMMs were used to investigate factors predicting body mass, WCC and plasticity therein. To investigate factors predicting body mass LMM1 was run with weight as the response variable, and context and grazing period were included as fixed effects. To investigate factors predicting WCC, LMM2 was run with cortisol as the response variable, body mass and age class were included as fixed effects to test for their effects on WCC (Ruis et al. 1997; Tilbrook 2000). Individual sheep ID was included as random effect in both models.

Using a combination of LMMs and linear models, I used a reaction norm approach (Nussey et al. 2007; Dingemanse et al. 2010) to assess whether the individual (I; random intercept), environmental change (E; being allowed to graze freely, referred to as 'context') or the interaction between the individual and environmental change (I x E; context as random slope) affected cortisol concentrations and performed ANOVA model comparisons using log-likelihood tests (Models 3-8; Bates \& Pinheiro 2000; Rieucau et al. 2010; Carter et al. 2012; Fürtbauer et al. 2015b). To obtain a measure of consistency between individual WCC samples the Intraclass Correlation Coefficient (ICC) was calculated using the R package ICC (Wolak et al. 2012). Finally, to test whether the level of plasticity was linked to initial wool cortisol values, a model (LMM9) was run with change in WCC between pre- and post-grazing samples as the response variable and pre-grazing WCC fitted as a fixed effect. Grazing period and percentage weight change in body mass between pre- and post-grazing sampling points were included as additional fixed effects. As this analysis compared individuals with different initial cortisol values, there was potential for regression toward the mean to occur (Barnett 2004; Kelly \& Price 2005). Therefore, I tested for a differential effect using the test for equality of variances in paired samples (equation 5; Kelly \& Price 2005). Individual sheep ID was included as a random effect in all models. Model 
assumptions of LMMs were checked using graphs of Q-Q plots and standardised residuals vs. fitted values.

\section{Results}

\section{Does sheep body mass increase in response to grazing (prediction 1)?}

Body mass was significantly lower before grazing compared to after grazing (LMM1: estimate $\pm \mathrm{SE}=-4.29 \pm 0.61, \mathrm{p}<0.001$; Table 1; Figure 2A), indicating that individuals, as predicted, gained weight during the grazing period. In addition, body mass was significantly larger in grazing period 2 than grazing period 1 (LMM1; estimate \pm SE $=3.70 \pm 0.63, \mathrm{p}<0.001$; Table 1). Body mass was also associated with age (LMM1; estimate $\pm \mathrm{SE}=-5.69 \pm 1.57, \mathrm{p}=0.001$; Table 2$)$, indicating that younger sheep $(3-4$ year olds) weighed less than older sheep (5-6 year olds).

Table 6.1: Factors predicting individual body mass $(\mathrm{kg})$ in $n=33$ sheep. Sheep ID was included as a random effect. Statistically significant results are shown in bold.

\begin{tabular}{llrr}
\hline Model & Predictor Variable & Estimate \pm SE & p-value \\
\hline LMM1 & & & \\
Body mass & & & \\
Fixed Effects & Intercept & $42.14 \pm 1.26$ & \\
& Grazing period 2 & $3.70 \pm 0.63$ & $<\mathbf{0 . 0 0 1}$ \\
& Pre-grazing & $-4.29 \pm 0.61$ & $<\mathbf{0 . 0 0 1}$ \\
& Age: 3-4 year olds & $-5.69 \pm 1.57$ & $\mathbf{0 . 0 0 1}$ \\
& & & \\
Random Effects & & $\chi^{\mathbf{2}}$ & $\boldsymbol{p}$-value \\
& Sheep ID & 44.95 & $<\mathbf{0 . 0 0 1}$ \\
\hline
\end{tabular}

Does sheep WCC change in response to grazing (prediction 2)?

WCC were significantly higher before than after grazing (LMM2: estimate $\pm \mathrm{SE}=$ $0.41 \pm 0.07, \mathrm{p}<0.001$; Table 6.2; Figure 6.2B), indicating that WCC decreased during grazing. There was a significant negative effect of grazing period (LMM2: estimate \pm $\mathrm{SE}=-0.21 \pm 0.07, \mathrm{p}=0.002$; Table 6.2; Figure 6.2B), with individuals having lower WCC in grazing period 2.

\section{Do individuals with heavier body mass have lower WCC (prediction 3)?}

WCC were significantly negatively associated with body mass (LMM2: estimate \pm SE $=-0.03 \pm 0.01, \mathrm{p}<0.001$; Table 6.2; Figure 6.2C), indicating that heavier individuals 
had lower WCC. Independent of body mass, younger sheep had lower WCC (LMM2: estimate $\pm \mathrm{SE}=-0.21 \pm 0.10, \mathrm{p}=0.049$; Table 6.2; Figure 6.2D).

Table 6.2: Factors predicting individual wool cortisol concentrations $(\mu \mathrm{g} / \mathrm{dL})$ in $n=33$ sheep. Sheep ID was included as a random effect in both models. Statistically significant results are shown in bold.

\begin{tabular}{llcr}
\hline Model & Predictor Variable & Estimate \pm SE & p-value \\
\hline LMM2 & & & \\
Log(Cortisol) & & & \\
Fixed Effects & Intercept & $4.24 \pm 0.33$ & \\
& Weight & $-0.03 \pm 0.01$ & $<\mathbf{0 . 0 0 1}$ \\
& Pre-grazing & $0.41 \pm 0.07$ & $<\mathbf{0 . 0 0 1}$ \\
& Grazing period 2 & $-0.21 \pm 0.07$ & $\mathbf{0 . 0 0 2}$ \\
& Age: 3-4 years old & $-0.21 \pm 0.10$ & $\mathbf{0 . 0 4 9}$ \\
Random Effects & & $\boldsymbol{\chi}^{\mathbf{2}}$ & $\boldsymbol{p}$-value \\
& Sheep ID & 10.19 & $\mathbf{0 . 0 0 1}$ \\
\hline
\end{tabular}

\section{Are there repeatable individual differences in WCC (prediction 4)?}

Cortisol concentrations ranged from $16.78 \mathrm{pg} / \mathrm{mg}-171.25 \mathrm{pg} / \mathrm{mg}$ (mean $\pm \mathrm{SD}=55.19$ $\pm 28.35 ; \mathrm{n}=120$ ). There was a significant effect of the individual and WCC were repeatable across the four sampling points $\left(\chi^{2}=14.08, \mathrm{p}<0.001\right.$, I; Table 6.3 , ICC $=$ $0.11,95 \% \mathrm{CI}=-0.05-0.31, \mathrm{p}=0.016)$, indicating individual consistency in WCC. Whilst there was a significant effect of individual and environment (prediction 2 above) there was no significant interaction between the individual and the environment (I x E; Table 6.3), indicating that whilst there was a population level decrease in WCC post-grazing, individual wool cortisol plasticity was not repeatable across the two grazing periods. Further analysis into cortisol plasticity revealed that the change in WCC was predicted by individuals' initial cortisol concentrations (LMM9: estimate \pm $\mathrm{SE}=-50.80 \pm 7.38, \mathrm{p}<0.001$; Table 6.4; Figure 6.2E), indicating that individuals with higher pre-grazing WCC showed a larger decrease in WCC during grazing. As this analysis compared individuals with different initial cortisol values, there was potential for regression toward the mean to occur (Barnett 2004; Kelly \& Price 2005). Using the equation described by Kelly \& Price (2005), I tested for and found evidence of a differential effect $(T=4.93, \mathrm{df}=32, \mathrm{p}<0.05)$, which suggests that the observed relationship between individuals pre-grazing WCC and cortisol change in response to grazing could have been a statistical rather than actual relationship between the variables (equation 5; Barnett 2004; Kelly \& Price 2005). Therefore, I constructed adjusted values and using these values showed that change in WCC was still predicted 
by individuals' initial cortisol concentrations (LMM10: estimate $\pm \mathrm{SE}=-31.72 \pm 6.90$, $\mathrm{p}<0.001$; Table 6.4; Figure 6.3).

Table 6.3: Comparison of LMMs with different fixed and random effects using an ANOVA test to compare log-likelihood ratios ( $n=120$ cortisol measures). Grazing period was included as a fixed effect in each model. Statistically significant results are shown in bold.

\begin{tabular}{lllllll}
\hline & Model & $\begin{array}{l}\text { Model } \\
\text { comparison }\end{array}$ & $\begin{array}{l}\text { Log- } \\
\text { likelihood }\end{array}$ & d.f & $\chi^{\mathbf{2}}$ & $\boldsymbol{P}$ \\
\hline I & M3: ID as a random intercept & M1 vs. M2 & -48.63 & 5 & 14.08 & $<\mathbf{0 . 0 0 1}$ \\
& & & -55.67 & 4 & & \\
M4: without ID & M3 vs. M4 & -48.63 & 5 & 45.26 & $<\mathbf{0 . 0 0 1}$ \\
& M5: context as a fixed effect & M6 & -71.26 & 4 & & \\
I x E & M7: without context & M5 vs. M6 & -48.51 & 7 & 0.24 & 0.887 \\
& intercept; context as a & & & & & \\
& random slope & & & & & \\
M8: without context as a & & -48.63 & 5 & & \\
& random slope & & & & \\
\hline
\end{tabular}

Table 6.4: Factors predicting cortisol change (LMM9) and adjusted cortisol change (LMM10) during two grazing periods in $n=33$ sheep. Sheep ID was included as a random effect in both models. Statistically significant results are shown in bold.

\begin{tabular}{llcc}
\hline Model & Predictor Variable & Estimate \pm SE & p-value \\
\hline LMM9 & & & \\
Cortisol change (pg/mg) & & & \\
Fixed Effects & Intercept & $183.85 \pm 32.04$ & \\
& (Log) Pre-Grazing Cortisol & $-50.80 \pm 7.38$ & $<\mathbf{0 . 0 0 1}$ \\
& Grazing period 2 & $1.15 \pm 6.62$ & 0.863 \\
& Weight change (\%) & $0.08 \pm 0.25$ & 0.767 \\
Random Effects & & $\chi^{\mathbf{2}}$ & $\boldsymbol{p}$-value \\
& Sheep ID & 0.79 & 0.373 \\
LMM10 & & & \\
Adjusted Cortisol change & Intercept & $122.44 \pm 30.10$ & \\
(pg/mg) Fixed Effects & (Log) Pre-Grazing Cortisol & $-37.72 \pm 6.90$ & \\
& Grazing period 2 & $6.02 \pm 6.71$ & \\
Random Effects & Weight change (\%) & $-0.14 \pm 0.25$ & \\
& & $\chi^{\mathbf{2}}$ & $\boldsymbol{p}$-value \\
& Sheep ID & 0.65 & 0.984 \\
\hline
\end{tabular}



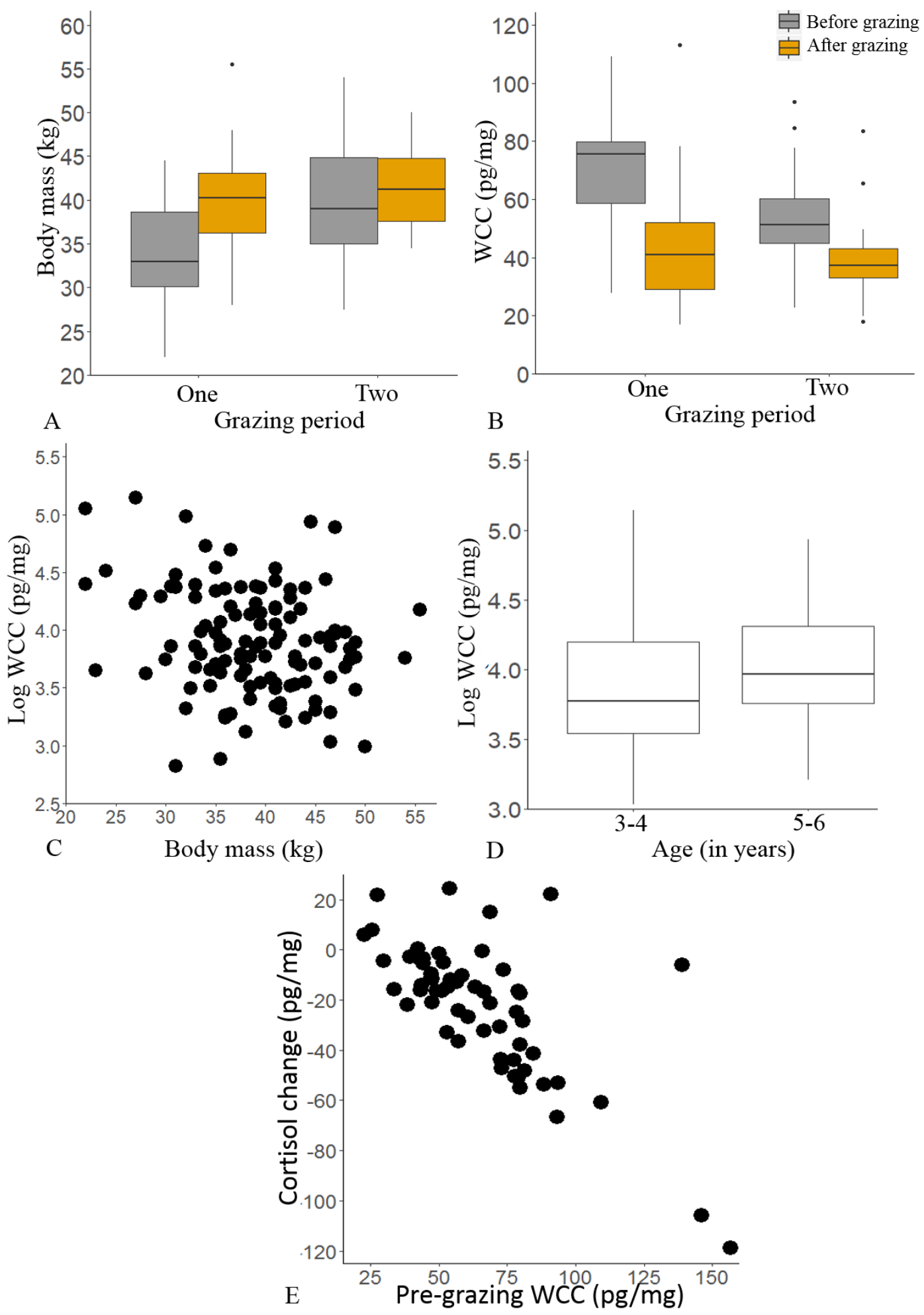

Figure 6.2: (A) Pre- and post-grazing body mass, (B) pre- and post-grazing WCC $(\mathrm{pg} / \mathrm{mg})$ in two grazing periods, $(\mathrm{C})$ relationship between individuals' body mass $(\mathrm{kg})$ and WCC (pg/mg), (D) differences in WCC between two age groups (3-4 and 5-6 years) and (E) relationship between pre-grazing cortisol (pg/mg) and wool cortisol change during the two grazing periods, $n=33$ sheep. Boxes $(A, B, D)$ indicate average value, first and third quartile values, whiskers indicate minimum and maximum values, and dots represent outliers. 


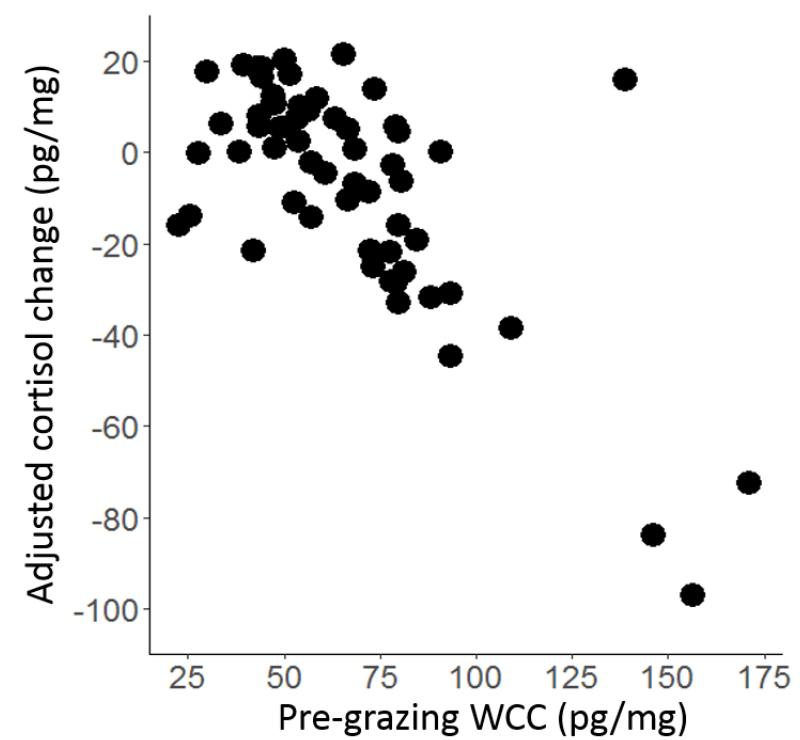

Figure 6.3: Relationship between pre-grazing cortisol (pg/mg) and adjusted wool cortisol change during the two grazing periods, $n=33$ sheep.

\section{Discussion}

Investigating endocrine plasticity over extended periods of time (e.g. months/years) is important to understand how animals may adapt to gradual environmental changes (Gabriel et al. 2005; Ghalambor et al. 2007). Exploring long-term HPA axis activity (i.e. the physiological stress response) in natural populations is difficult because obtaining repeated samples is not always possible. Here, I used domestic mountain sheep to investigate the repeatability and plasticity of wool cortisol across two 1-month grazing periods where individuals were able to graze freely in an upland pasture. I also investigated the link between pre-grazing WCC and level of wool cortisol plasticity during grazing, along with factors predicting WCC (weight, pre-grazing cortisol, second grazing period cortisol, and age) and body mass (pre-grazing weight, second grazing period weight, and age). Below, I discuss each finding in turn.

First, as predicted, results showed that individuals' pre-grazing body mass was lower, showing that individuals gained weight during grazing as a result of being able to graze freely on high quality food during the 1-month grazing period. This is because during periods were resources are increased individuals will increase food intake to store energy as fat and thus increase their body mass (Baile \& Forbes 1974; Forbes 1977). Therefore, from an ecological perspective, it is assumed that in times of plentiful food resources individuals would be able to feed and increase body mass for potential future periods of lower food availability (Buchanan \& Partecke 2012). 
Second, individuals showed significantly higher WCC pre-grazing, meaning WCC decreased during each grazing period (population level plasticity). I also found that WCC was negatively associated with body mass, suggesting that individuals that weighed more had lower HPA axis activity, potentially as they are able to consume more food and store excess energy in the form of fat (Forbes 1977). Research has shown that a decrease in food availability is associated with elevated cortisol levels in red colobus monkeys (Chapman et al. 2015), ring-tailed lemurs (Fardi et al. 2018), Guatemalan black howler monkeys (Alouatta pigra, Behie et al. 2010), polar bears (Kroshko et al. 2017), black-legged kittiwakes (Wingfield \& Kitaysky 2002), song birds (Clinchy et al. 2004) and white ibis (Herring et al. 2011). Individuals use energy for daily metabolic process, which they get from food intake they have consumed (Nelson 2011). In natural environments, changes in food resource occur naturally with seasonal changes (Buchanan \& Partecke 2012), and in periods of diminished resources individuals will need to conserve their energy as they might not know when resources will increase: this can causes the HPA axis to be activated (Caslini et al. 2016). In this study, food was not a limiting factor due to individuals being able to freely graze. As a result of individuals being able to consume unlimited food, there would be no activation of the HPA axis as energy for metabolic process would be plentiful. Individuals were also able to store excess energy as adipose tissue and cortisol production was decreased as a result of not being in a stressful environment. In addition to research showing that a decrease in food availability is associated with elevated cortisol levels, it has also been shown that introducing food to a poor-resource environment can decrease cortisol concentrations (Ashley 2007). The relationship between WCC and body mass that I find here therefore suggests that in wild animals WCC (or hair cortisol concentrations) can be used as a marker of body conditions, i.e. nutritional status. However, it is important to mention that human research has focused on the relationship between obesity and elevated cortisol concentrations, with studies showing that cortisol increases with increased body mass/BMI/waist-to-hip ratio (Wallerius et al. 2003; Manenschijn et al. 2011b; Stalder \& Kirschbaum 2012). This suggests that the relationship between cortisol and body mass is not straight forward and that perhaps at both extremes (low and high food availability) individuals HPA axis can be activated. 
WCC was also associated with age, with younger individuals having lower WCC. The relationship between cortisol and age is complex, and studies have shown mixed results depending on species (Comin et al. 2012; Terwissen et al. 2013; Carlitz et al. 2014; Dettmer et al. 2014; Heimbürge et al. 2020). It is suggested that younger individuals have higher plasma cortisol as a result of lower corticosteroid binding globulin levels (Heimbürge et al. 2020). Research has supported this by showing that older individuals had lower cortisol concentrations in hair of horses (Equus ferus caballus, Comin et al. 2012; Montillo et al. 2014), rhesus monkeys (Dettmer et al. 2014), vervet monkeys (Laudenslager et al. 2012) and guinea baboons (Papio papio, Fourie \& Bernstein 2011), some studies have found no differences in hair cortisol concentration, e.g. orangutans (Pongo spp, Carlitz et al. 2014), grizzly bears (Ursus arctos horribilis, Macbeth et al. 2012), Canada lynx (Lynx canadensis, Terwissen et al. 2013) and domestic dogs (Roth et al. 2016). In line with my findings it has been shown that older pigs (sows compared to piglets) had higher cortisol concentrations (Heimbürge et al. 2020). Additionally, in humans, adults have been shown to have higher cortisol concentrations compared to infants (Dettenborn et al. 2012; Feller et al. 2014). The results from this study therefore indicate the importance of including age in analysis as the effect of age on cortisol levels are species-specific (Heimbürge et al. 2018). The time frame of age comparisons might also be important, as some research compare days (Montillo et al. 2014), months (Fourie \& Bernstein 2011; Comin et al. 2012) or years (Fourie \& Bernstein 2011; Dettenborn et al. 2012; Dettmer et al. 2014; Feller et al. 2014) and it is possible that age related increases and decreases occur differently at different age comparisons. In my study, individuals were grouped into two appropriate age groups with only three years difference, and therefore my result represents only a relatively small age difference. It would be interesting to investigate the relationship across a wider range of ages from newborn to adult sheep to see the full effect of age.

Finally, I found repeatability in individual WCC across the four sampling points, indicating repeatability in long-term HPA axis activity which is in line with previous work showing individual cortisol consistency (Narayan et al. 2013; Fürtbauer et al. 2015b; Madliger \& Love 2016; Cockrem et al. 2017). Whilst there was a population level WCC decrease during the grazing periods (environment effect) and consistent individual differences (individual effect), there was no interaction between the 
environment and the individuals, meaning that whilst all individuals decreased WCC in both grazing periods the individual WCC decrease was not consistent (repeatable) across the two grazing periods. Additionally, whilst there was an environmental effect on the population, I found that individuals' pre-grazing WCC were related to the level of wool cortisol plasticity, with individuals that showed higher initial WCC showing greater plasticity, i.e. a larger decrease in WCC. This could suggest an adaptive value of the endocrine system in response to nutritional status. In relation to my results, it would suggest that the individuals with greater pre-grazing cortisol concentrations are more responsive to environmental changes such as changing food availability, although more controlled nutritional conditions would explore this future. In theoretical framework reviews it has been suggested that individuals that are more flexible and able to respond to environmental changes have greater fitness (Gabriel et al. 2005; Sih et al. 2011; reviewed by Taff \& Vitousek 2016), future work is needed to investigate whether individuals with higher pre-grazing cortisol are better able to respond to environmental changes (i.e. are individuals with higher initial cortisol quicker to respond to predators).

Generally, hormones link the environment and the genome, and the same environment can be interpreted and produce a range of phenotypes (reviewed by Dufty et al. 2002). This could explain the difference in pre-grazing cortisol concentrations among individuals - those that interpreted lower nutritional value of food had increased cortisol concentrations (Dufty et al. 2002). Once the energy requirements where met (through increased food consumption), the individual's cortisol decreased which explains the overall decrease in cortisol concentrations during the grazing periods. Additionally, as environmental conditions varied between the two pre-grazing periods, this could account for the lack of an environmental and individual interaction and suggest that it is the individuals' current environment that affects endocrine plasticity ( $\mathrm{I}$ E E). This further adds to the understanding of endocrine plasticity as it suggests that levels of plasticity are not only dependent on the individual but also the environmental conditions (Dufty et al. 2002).

\section{Conclusion}

Overall, this study shows that, in a population of Welsh mountain sheep, repeated WCC measures can be used to assess individual and population endocrine plasticity 
and changes in body mass to changing food availability over time. When resources are freely available individuals increase body mass, likely an indicator of storing excess food as mass in natural environments where food availability can fluctuate (Buchanan \& Partecke 2012). WCC also decreases during grazing, an indicator of a lack of HPA axis activity when resources are freely available (Ashley 2007; Nelson 2011). Interestingly, cortisol plasticity was predicted by pre-grazing WCC, which could suggest these individuals are potentially more reactive to environmental changes, in this case more reactive to plentiful food resources, by increasing body mass, thus by being more reactive and flexible and potentially having fitness advantages. Taken together these results suggest that WCC can be used as an indicator of sheep nutritional status and show that endocrine plasticity can be depend on the current environmental conditions an individual is in. Future research should look at whether individuals that are more flexible in WCC changes to food availability are also more flexible/reactive when experiencing other environmental changes such as the effects of climate change. 


\section{Chapter 7: General Discussion}

Endocrine plasticity is the reversible change in individual endocrine traits in response to unpredicted physical or social environmental changes (reviewed by Taff \& Vitousek 2016), and being able to respond to and cope with environmental changes is crucial for survival (Windig et al. 2004; Gianoli \& Valladares 2012; reviewed by Taff \& Vitousek 2016). There is a growing body of literature showing endocrine plasticity associated with changes to the physical environment (e.g. novel environment; Hennessy et al. 2009; Colson et al. 2012; Miranda-de la Lama et al. 2012; Fürtbauer \& Heistermann 2016), changes in food availability (Blom et al. 2000; Wingfield \& Kitaysky 2002; Clinchy et al. 2004; Kitaysky et al. 2007; Behie et al. 2010; Herring et al. 2011; Chapman et al. 2015; Fardi et al. 2018), changes to social dynamics (e.g. social isolation; Dronjak et al. 2004; Ruscio et al. 2007; Banerjee \& Adkins-Regan 2011; Ferland \& Schrader 2011; Heimbürge et al. 2018) and interactions with unfamiliar individuals (Castro \& Matt 1997; Woodley et al. 2000). Much less is known about individual variation and repeatability in endocrine plasticity. Why does the variation exist? Is this variation consistent and does it influence the expression/plasticity of other (e.g. behavioural or morphological) traits? If these questions can be answered, and the causes and consequences of variation in endocrine plasticity are known, perhaps it can help researchers understand why some individuals and/or populations are able to cope and thrive under environmental changes, whilst others are not (reviewed by Taff \& Vitousek 2016). In this thesis, I addressed five specific research questions (see Table 7.1) and here, I summarise my key findings in relation to each of these research questions and place them in a wider context. 
Table 7.1: Summary of research questions addressed in each data chapter. Green ticks indicate evidence, red crosses indicate absence of evidence (empty box indicates research question not addressed).

\begin{tabular}{|l|c|c|c|c|}
\hline Research question & $\begin{array}{c}\text { Chapter 3 } \\
\text { (three-spined } \\
\text { sticklebacks) }\end{array}$ & $\begin{array}{c}\text { Chapter 4 } \\
\text { (three-spined } \\
\text { sticklebacks) }\end{array}$ & $\begin{array}{c}\text { Chapter 5 } \\
\text { (human) }\end{array}$ & $\begin{array}{c}\text { Chapter 6 } \\
\text { (Welsh } \\
\text { mountain sheep) }\end{array}$ \\
\hline $\begin{array}{l}\text { 1. Are individuals } \\
\text { repeatable in their } \\
\text { endocrine traits? }\end{array}$ & $\checkmark$ & & \\
\hline $\begin{array}{l}\text { 2. Do individuals show } \\
\text { plasticity in endocrine } \\
\text { traits ('endocrine } \\
\text { plasticity') and are they } \\
\text { repeatable therein? }\end{array}$ & $\mathrm{X}$ & & $V^{*}$ & $\checkmark^{* *}$ \\
\hline $\begin{array}{l}\text { 3. Does between- } \\
\text { individual variation in } \\
\text { endocrine traits predict } \\
\text { plasticity therein? }\end{array}$ & $\mathrm{X}$ & & $\checkmark$ & \\
\hline $\begin{array}{l}\text { 4. Does between- } \\
\text { individual variation in } \\
\text { endocrine traits relate to } \\
\text { behaviour/morphological } \\
\text { condition? }\end{array}$ & & & & \\
\hline $\begin{array}{l}\text { 5. Does the endocrine } \\
\text { status of social partners } \\
\text { affect endocrine } \\
\text { plasticity? }\end{array}$ & $\mathrm{X}$ & & & \\
\hline
\end{tabular}

*Endocrine plasticity measured, but due to experimental set up repeatability in plasticity was not assessed in this chapter

**Repeatability in endocrine plasticity measured, but results show no repeatability in endocrine plasticity

\section{Are individuals repeatable in their endocrine traits?}

Repeatability in endocrine traits is important to investigate between-individual variation in these endocrine traits (Schoenemann \& Bonier 2018). Repeatability in endocrine traits enables researchers to understand individual endocrine profiles and exploring whether repeatability occurs in response to environmental changes can shed light on how individuals cope with challenges (i.e. if endocrine repeatability is consistent across environmental contexts this can be used to predict an individual's response to change; Schoenemann \& Bonier 2018). Previous research has shown repeatability in short-term glucocorticoid measures in various species including birds (Romero \& Reed 2008; Cockrem et al. 2009; Rensel \& Schoech 2011), fish (Bell et al. 2010; Cook et al. 2011; Fürtbauer et al. 2015b; Samaras et al. 2016; Bensky et al. 
2017), and mammals (Koren et al. 2012; Sonnweber et al. 2018; Fürtbauer et al. 2019). Long-term glucocorticoid repeatability has been shown in humans (Raul et al. 2004; Burnard et al. 2017) and non-human primates (Carlitz et al. 2014, 2015; Grant et al. 2017).

I found evidence for repeatability in short-term endocrine traits, i.e. waterborne cortisol concentrations in stickleback fish (Chapter 3), cortisol and testosterone concentrations in humans (Chapter 5), as well as long-term endocrine traits, i.e. wool cortisol concentrations (Chapter 6). The results in this thesis further demonstrate endocrine repeatability across different species and contexts, including physical environment contexts (Chapter 3, 5 \& 6) and social environment context (Chapter 3). Taken together, my findings on repeatability in short- and long-term HPA axis activity, adds to the literature that endocrine measures should be considered a 'personality trait' (Fürtbauer et al. 2015b). This is important because individual differences in endocrine traits may be linked to variation in endocrine plasticity and other behavioural and morphological traits (reviewed by Taff \& Vitousek 2016). For example, individuals with consistently higher endocrine traits could show greater or lesser endocrine plasticity in response to an environmental change. If variation in endocrine traits predicts variation in endocrine plasticity and other traits, it could help researchers understand and predict how individuals will respond to specific environmental changes, such as climate change.

\section{Do individuals show plasticity in endocrine traits ('endocrine plasticity') and are they repeatable therein?}

Endocrine plasticity is the reversible change in endocrine traits in response to unpredicted environmental changes (Taff \& Vitousek 2016). Depending on the environmental gradient, endocrine plasticity can be short- or long-term, taking place within minutes or hours (e.g. social interaction) or over weeks or months (e.g. changing food availability; DeWitt \& Scheiner 2004; Windig et al. 2004; Noh et al. 2017). Previous research has shown endocrine plasticity in response to novel environments (Hennessy et al. 2006; Colson et al. 2012; Miranda-de la Lama et al. 2012), changes in the social environment (Liebgold \& Dibble 2011; Galhardo et al. 2012) and changes in food availability (Blom et al. 2000; Chapman et al. 2015; Fardi et al. 2018). However, repeatability in endocrine plasticity is often difficult to 
investigate as repeated samples are hard to obtain (reviewed by Burnard et al. 2017; Taff et al. 2018).

My results show evidence for a non-significant trend in cortisol plasticity in response to a social task (in humans; Chapter 5) and no cortisol plasticity in response to novel environments (in stickleback fish; Chapter 3). I did find plasticity in wool cortisol concentrations (WCC) in response to changing food availability (in sheep; Chapter 6). Taken together, these results further add to the current knowledge about under which environmental condition plasticity occurs. Interestingly, in my studies, the long-term measure of cortisol (WCC) showed significant plasticity, whereas it was not significant in the short-term measures of cortisol. Perhaps repeatability in plasticity differs between short- and long-term cortisol measures, in addition to varying environments. Short-term cortisol measures, such as saliva, are subject to hourly fluctuations (Dahlgren et al. 2009; Matsuda et al. 2012), which could suggest why plasticity in short-term cortisol measures over hourly time-periods are not significantly different. Future work should investigate both short- and long-term plasticity in the same species under different environmental conditions to see if repeatability in endocrine plasticity, occurs in both long-term and short-term measures or only in long-term measures (i.e. hair/wool cortisol concentration).

\section{Does between-individual variation in endocrine traits predict plasticity therein?}

Previous theoretical work has stated that there should be individual differences found in baseline glucocorticoids and plasticity responses (Guindre-Parker 2020). This is due to baseline glucocorticoids maintaining homeostasis, which is expected to be different for each individual (as individuals perceive stressors differently, which results in varying levels of glucocorticoids being released), and therefore, it would be expected that individuals optimally shift their energetic requirements when environmental conditions change (Madliger \& Love 2016; Guindre-Parker 2020). Individual variation in baseline glucocorticoids and plasticity have been shown in various species including chimpanzees (Pan troglodytes, Anestis et al. 2006), red squirrels (Sciurus vulgaris, Guindre-Parker et al. 2019), beluga whales (Delphinapterus leucas, Schmitt et al. 2010), blue tits (Parus caeruleus, Müller et al. 2006), tree swallows (Tachycineta bicolor, Madliger \& Love 2016) and flatfish (Solea senegalx, Silva et al. 2010). Theoretical arguments suggest that individuals with more flexible slopes (e.g. greater 
flexibility/plasticity) are better able to respond to a dynamic environment (reviewed by Taff \& Vitousek 2016). In my studies, individuals with higher baseline cortisol measures showed greater plasticity, more specifically (a greater decrease their HPA/I axis activity). Whilst increases in HPA/I axis activity are adaptive in stressful environments (i.e. to heighten responses and relocate energy where needed), chronic increases are maladaptive (i.e. can decrease growth Nelson 2011). Therefore, the decrease in glucocorticoids in response to a non-stressful environment is adaptive as it ensures individuals do not become chronically stressed (Tsigos et al. 2000; Nelson 2011; Stephens \& Wand 2012). In my studies, the decrease in response to 'non stressful' environments could represent that pre-conditions were potentially stressful (i.e. anticipation of a social task in humans and less enriching environment in sheep). Increases and decreases in glucocorticoids in response to varying environmental conditions are vital for survival and enables individuals to redirect energy for essential processes (Nelson 2011). This process of maintaining internal physiological homeostasis in response to the changing perceived and actual demands of changing external environments is known as allostasis (McEwen \& Wingfield 2010; Maestripieri \& Hoffman 2011; Nelson 2011).

Considering the Reactive Scope Model, proposed by Romero et al., (2009), which builds on the allostasis concept (McEwen \& Wingfield 2010; Maestripieri \& Hoffman 2011) it suggests four ranges which glucocorticoids fall into when responding to stress. In summary there is (1) predictive homeostasis (the circadian and seasonal variation in concentrations to predictable environmental changes, i.e. seasonal weather changes) and (2) reactive homeostasis (range of concentrations in response to unpredictable environmental changes, i.e. predators), and as individuals will encounter predictable and unpredictable environmental changes daily, the predictive and reactive homeostasis relates to an individual's reactive scope (Romero et al. 2009). Therefore, when individuals respond to physical and social environmental changes, there cortisol plasticity reflects their reactive scope range (Romero et al. 2009). However, when concentrations go above this reactive scope this is (3) homeostatic overload and when it falls below it is (4) homeostatic failure (Romero et al. 2009). In individuals that are already operating at a higher glucocorticoid concentration (i.e. individuals with higher baseline glucocorticoid concentrations in my studies), it would be interesting to know if these individuals would be more plastic and increase glucocorticoids in response to 
a physiologically stressful environment. According to the reactive scope model, if an individual's physiological concentration goes above their reactive scope this would place them in homeostatic overload which has negative effects (Romero et al. 2009). Therefore, if these individuals with higher baseline glucocorticoids are at the top range of their reactive scope, further increases could push them into homeostatic overload which could have negative effects on individuals (Romero et al. 2009). However, it could be that individuals with higher baseline levels within my studies generally have higher upper reactive scope range. To fully understand an individual's reactive scope and under which conditions individuals fall out of their reactive scope, multiple baseline cortisol measures, and measures after various environmental changes (i.e. 'stressful' and 'non-stressful') would need to be taken.

\section{Does between-individual variation in endocrine traits relate to behaviour/morphological condition?}

Coping styles, i.e. coherent sets of physiological and behaviour traits (Koolhaas et al. 1999; Coppens et al. 2010), occur in various vertebrate species, including e.g. Barbary macaques (Macaca sylvanus, Tkaczynski et al. 2019), great tits (Parus major, Carere \& Van Oers 2004; Baugh et al. 2012), zebrafish (Danio rerio, Tudorache et al. 2013, 2015), brown trout (Salmo trutta ,Brelin et al. 2005) and three-spined stickleback fish (Gasterosteus aculeatus, Bensky et al. 2017). Proactive and reactive coping styles are typically found in 'stressful' environments and present two ways that individuals can cope with a challenge; proactive individuals show low HPA axis activity, high physical activity and aggression, and are behaviourally less flexible whilst reactive individuals show high HPA axis activity, immobility and avoidance behaviour and are behaviourally more flexible (Koolhaas et al. 1999; Coppens et al. 2010). In my study on sticklebacks (Chapter 4), I found a significant negative correlation between activity (distance travelled) and average individual waterborne cortisol concentrations, indicating that individuals with higher average HPI axis activity were less active, in a novel but 'non-stressful' environment (i.e. a novel environment that did not increase HPI axis activity). This suggests that coping styles can be found also in non-stressful contexts, which adds to the current literature which typically reports coping styles exclusively in stressful situations (Liu et al. 2013; Saxbe et al. 2015; Fürtbauer \& Heistermann 2016). Perhaps, this suggests that coping styles reflect how individuals 'cope' with varying conditions more generally and not just in stressful contexts. 
Whilst coping styles have been studied widely in non-human animals (Carere \& Van Oers 2004; Brelin et al. 2005; Baugh et al. 2012; Tudorache et al. 2015; Bensky et al. 2017; Tkaczynski et al. 2019), 'coping styles' research in humans tends to be called 'personality research', with personality obtained via questionnaires (Dall et al. 2004; Nettle \& Penke 2010). Big 5 personality traits are commonly used in human personality research to assess individual personalities (Goldberg 1990, 1999; Bibbey et al. 2013). Research has shown that neuroticism and extraversion are correlated with increased cortisol and that high conscientiousness scores correlated with decreased cortisol under normal conditions (i.e. taking daily measures LeBlanc \& Ducharme 2005; Nater et al. 2010; Hill et al. 2013; Steptoe et al. 2017). Under stressful conditions (e.g. undergoing laboratory stress test) lower openness, lower agreeableness and higher neuroticism has been shown to be associated with lower cortisol (Oswald et al. 2006; Bibbey et al. 2013). In Chapter 5, I found that the personality trait conscientiousness was positively correlated with average salivary cortisol concentrations. This result further adds to the mix of results showing different relationships between cortisol and the Big 5 personality traits (Oswald et al. 2006; Nater et al. 2010; Bibbey et al. 2013; Hill et al. 2013; Steptoe et al. 2017). Perhaps, to fully understand how cortisol and personality traits interact in humans and whether certain traits 'help' individuals 'cope' with varying environments I suggest that a longitudinal study on multiple individuals across varying environmental contexts (i.e. stressful and non-stressful) is needed.

In addition to between-individual variation in the links between endocrine and behavioural traits, in Chapter 6, I investigated links between HPA-axis activity and morphology and found that sheep wool cortisol concentrations were negatively associated with body mass. Whilst an increase in cortisol when food is limited has been found in species (Blom et al. 2000; Wingfield \& Kitaysky 2002; Behie et al. 2010; Chapman et al. 2015; Fardi et al. 2018) and a decrease in cortisol when food is added in artificial environments (Fokidis et al. 2012), to my knowledge there was no previous research looking into the relationship between changing food availability and cortisol responses in natural environments. This result highlights plasticity in cortisol and morphology in response to natural increases in food availability, and relationships between endocrine and morphological traits. This is interesting as it suggests that between-individual variation in endocrine traits not only reflects how an individual 
might cope with different contexts, but also reflects individual variation in morphological condition.

Taken together, my results show between-individual variation in glucocorticoid baseline measures, plasticity responses and between-individual variation in links between endocrine measures and behaviour/morphological traits, which supports the theory that populations should include individual differences (Madliger \& Love 2016; Guindre-Parker et al. 2019; Guindre-Parker 2020). These individual differences provide support for the evolutionary "optimal endocrine hypothesis", which proposes that individual variation in endocrine traits is generated through expression of nearoptimal endocrine phenotypes to overcome environmental change (Bonier \& Cox 2020). Variation in phenotypic expression exists because optimal endocrine response will be different for each environmental change. Therefore, it would be advantageous for individuals to respond differently depending on the environmental change, which could explain why between-individual variation exists within populations. Whilst the results from this thesis show between-individual variation in glucocorticoid baseline and plasticity in response to different environmental conditions along with betweenindividual variation in behaviour and morphology, it would be interesting to conduct a longitudinal study on the same species exploring various environmental conditions to see the full effects of between-individual variation.

\section{Does the endocrine status of social partners affect endocrine plasticity?}

Glucocorticoid responses are known to be affected by changes in the social environment (DeVries et al. 2003). For example, interactions with unfamiliar individuals (Castro \& Matt 1997; Woodley et al. 2000) and social isolation can lead to increased glucocorticoids (Dronjak et al. 2004; Ruscio et al. 2007; Banerjee \& Adkins-Regan 2011; Ferland \& Schrader 2011; Heimbürge et al. 2018). Dyad partners can also bidirectionally influence each other's glucocorticoid concentrations, i.e. via cortisol coregulation, which has been shown between familiar dyads in humans (Sbarra \& Hazan 2008; Saxbe \& Repetti 2010; Liu et al. 2013; Buttner et al. 2015) dogs (Cunningham 2017), and stickleback fish in response to a novel, stressful environment (as indicated by increases in cortisol concentrations; Fürtbauer \& Heistermann 2016). In my study, I found no evidence of cortisol coregulation (Chapter 3), perhaps due to cortisol coregulation only occurring in response to a stressful environment (Fürtbauer 
\& Heistermann 2016), which was not the case in my study (cortisol did not increase in response to the novel environment). Cortisol coregulation functions to reduce or inhibit the stress response of a social partner under conditions that they would normally perceive as stressful (Sbarra \& Hazan 2008; Saxbe \& Repetti 2010) which may explain the absence of coregulation in my study.

\section{Conclusion}

The overarching aim of my thesis was to investigate individual differences and plasticity in endocrine traits in different vertebrate systems and, the findings from my research give several insights into this area. In this thesis, I show repeatability in both short-term and long-term endocrine traits in response to changes in both the physical (novel environment and food availability) and social environments (social tasks; conspecific familiarity and housing conditions) in three different vertebrate systems. My findings complement previous results indicating traits are repeatable (Rensel \& Schoech 2011; Fürtbauer et al. 2015b, 2019; Bensky et al. 2017; Burnard et al. 2017; Sonnweber et al. 2018), hence can and should be considered a personality trait (Fürtbauer et al. 2015b). The wider implications of repeatability of glucocorticoids is that they can be used to understand (i) the way individuals cope with a challenge and (ii) whether natural selection can influence these traits (reviewd by Schoenemann $\&$ Bonier 2018). The results from my studies hint at short-term plasticity (i.e. plasticity in salivary cortisol in response to a social task) and show long-term plasticity (i.e. plasticity in wool cortisol concentrations), and suggests that long-term cortisol plasticity might be more repeatable than short-term plasticity; which could be due to fluctuations in short-term measures (which could be hourly; Russell et al. 2012; Caslini et al. 2016). The fluctuations are a result of daily diurnal cortisol rhythms, and short-term measures could be 'masking' short-term plasticity on occasions (Russell et al. 2012). My results did not show repeatability in either short- or long-term plasticity. My results do show long-term plasticity in response to a physical environmental change (i.e. wool cortisol in response to changing food availability).

My findings on between-individual differences in endocrine traits support theoretical suggestions that individuals with higher baseline glucocorticoids are more flexible (Taff \& Vitousek 2016); as individuals with higher baseline cortisol concentrations 
exhibited larger changes (i.e. were more flexible). This suggests that betweenindividual variation in endocrine traits does predict plasticity therein; which has future implications for understanding what physiological conditions enable individuals to cope with environmental change (i.e. whether certain levels of endocrine baseline traits enable individuals to overcome challenges), which ultimately increases survival (reviewed by Schoenemann \& Bonier 2018). Furthermore, my studies provide evidence for a relationship between inter-individual variation in endocrine traits and behaviour and morphological condition, respectively. I found evidence for coping styles (reviewed by Koolhaas et al. 1999; Coppens et al. 2010) in three-spined stickleback fish in a non-stressful environment and some evidence for copying styles in humans (i.e. a link between cortisol and the personality trait consciousness). Finally, whilst effects of social partners on endocrine status have been shown previously (e.g. social buffering/cortisol coregulation; Bartolomucci et al. 2003; DeVries et al. 2003; Rukstalis \& French 2005; Sbarra \& Hazan 2008; Hennessy et al. 2009; Papp et al. 2013; Mörelius et al. 2015; Saxbe et al. 2015; Fürtbauer \& Heistermann 2016), my work on sticklebacks did not show coregulatory effects on HPA-axis activity. This further adds to the suggestion that the endocrine status of social partners only affects plasticity in stressful environments (i.e. environments that cause an increase in HPA/I axis activity; Liu et al. 2013; Saxbe et al. 2015; Fürtbauer \& Heistermann 2016). Under these conditions, the presence of a partner could be beneficial and correlating glucocorticoids could be a way for individuals to cope with the environmental change.

\section{Future directions}

Based on my results investigating my overarching research aims, I suggest the following future research directions:

- Further investigations into repeatability in plasticity. Whilst my results show some plasticity, I was unable to explore repeatability in plasticity in each environment. I suggest purposely designing repeated measures studies that enable exploration of phenotypic plasticity using both short- and long-term measures of plasticity in different species (e.g. saliva and hair cortisol measures in humans in response to social tasks, or waterborne cortisol measures in stickleback fish to foraging or predators). This would help untangle whether 
endocrine plasticity occurs under different environmental conditions, and whether plasticity occurs in both short- and long-term measures of cortisol.

- Further investigation into between-individual variation in endocrine traits predicting plasticity therein. It would be interesting to explore whether individual baseline endocrine traits predict plasticity across different environmental contexts. My results highlight that baseline endocrine traits can predict plasticity in one environmental context, but it would be interesting to investigate whether this is consistent across contexts (both 'stressful' and 'nonstressful' environmental changes).

- Investigations into whether social partners only affect focal individuals' endocrine traits under 'stressful' environmental conditions, i.e. those that cause an increase in HPA/I axis activity. My results hint that under 'non-stressful' conditions there is no effect of social partners, however, as my result is only in one species in response to one environmental condition further, work could explore this in more species and across contexts. 


\section{Chapter 8: Supplementary material}

\section{Appendix 1: Supplementary material for Chapter 3}

Table 8.1: Summary of LMMs exploring potential cortisol coregulation in stickleback fish dyads ( $\mathrm{n}=36$ dyads) for post-NE cortisol measurements, and potential interactions between recent social context and familiarity. Note, both cortisol and partner cortisol were $\log$ transformed (see methods for details). Initially, the model suggested an interaction between housing and partner cortisol (LMM6), but when the nonsignificant familiarity and partner cortisol interaction was removed the interaction was no longer significant (LMM7). Fish ID and Dyad ID were included as random effects. Statistically significant results are shown in bold.

\begin{tabular}{|c|c|c|c|c|}
\hline Model & $\begin{array}{l}\text { Response } \\
\text { Variable }\end{array}$ & Predictor Variable & Estimate \pm s.e. & $p$-value \\
\hline $\begin{array}{l}\text { LMM6 } \\
\text { Post-NE }\end{array}$ & $\begin{array}{l}\text { Cortisol } \\
\text { (ng/g/h) }\end{array}$ & $\begin{array}{l}\text { Intercept } \\
\text { Partner cortisol } \\
\text { Unfamiliar } \\
\text { Housing solitary } \\
\text { Sex Male } \\
\text { Time: PM } \\
\text { Partner cortisol: unfamiliar } \\
\text { Partner cortisol: housing } \\
\text { solitary }\end{array}$ & $\begin{array}{l}0.56 \pm 0.18 \\
0.06 \pm 0.15 \\
-0.11 \pm 0.22 \\
-0.06 \pm 0.22 \\
-0.88 \pm 0.20 \\
-0.20 \pm 0.18 \\
0.18 \pm 0.21 \\
-0.39 \pm 0.18\end{array}$ & $\begin{array}{r}0.680 \\
0.626 \\
0.767 \\
<\mathbf{0 . 0 0 1} \\
0.263 \\
0.385 \\
\mathbf{0 . 0 3 7}\end{array}$ \\
\hline $\begin{array}{l}\text { LMM7 } \\
\text { Post-NE }\end{array}$ & $\begin{array}{l}\text { Cortisol } \\
\text { (ng/g/h) }\end{array}$ & $\begin{array}{l}\text { Intercept } \\
\text { Partner cortisol } \\
\text { Housing solitary } \\
\text { Sex Male } \\
\text { Time PM } \\
\text { Partner cortisol: housing } \\
\text { solitary }\end{array}$ & $\begin{array}{r}0.50 \pm 0.14 \\
0.16 \pm 0.10 \\
-0.11 \pm 0.19 \\
-0.86 \pm 0.20 \\
-0.21 \pm 0.17 \\
-0.32 \pm 0.16\end{array}$ & $\begin{array}{r}0.107 \\
0.551 \\
<\mathbf{0 . 0 0 1} \\
0.240 \\
0.052\end{array}$ \\
\hline
\end{tabular}

Table 8.2: Summary of effect of individual and partner cortisol concentration on changes in cortisol concentrations from pre-NE to post-NE1. Note pre-NE and partner pre-NE cortisol was logged transformed. Fish ID and Dyad ID were included as random effects. Statistically significant results are shown in bold.

\begin{tabular}{lllrr}
\hline Model & Response Variable & Predictor Variable & Estimate \pm s.e. & p-value \\
\hline LMM5 & Cortisol change & Intercept & $191.43 \pm 72.96$ & \\
& & Pre-NE cortisol & $-265.77 \pm 59.17$ & $<\mathbf{0 . 0 0 1}$ \\
& Partner pre-NE & $22.14 \pm 56.24$ & 0.695 \\
& cortisol & & \\
Random & Sex Male & $-280.05 \pm 131.76$ & $\mathbf{0 . 0 3 8}$ \\
Effects & & $\chi^{\mathbf{2}}$ & $\boldsymbol{p}$-value \\
& & & 0.75 & 0.386 \\
\hline
\end{tabular}


Table 8.3: Model output of post hoc analysis ran to investigate whether housing individual socially or solitarily affected change in cortisol. Cortisol change (log transformed) was the response and an interaction between pre-NE cortisol and housing was included. Group ID was included as a random effect.

\begin{tabular}{llcc}
\hline Response Variable & Predictor Variable & Estimate \pm s.e. & p-value \\
\hline (Log)Cortisol Change & & & \\
Fixed effects & Intercept & $4.63 \pm 0.59$ & \\
& (Log)Pre-NE & $-0.97 \pm 0.25$ & $<0.001$ \\
& Housing Social & $-0.81 \pm 0.66$ & 0.228 \\
& (Log)Pre-NE:Housing Social & $0.34 \pm 0.27$ & 0.221 \\
Random effects & & $\boldsymbol{\chi}^{\mathbf{2}}$ & p-value \\
& Group ID & 0.00 & 1 \\
\hline
\end{tabular}




\section{Appendix 2: Supplementary material for Chapter 4}

Table 8.4: Repeatability of (i) total distance travelled, (ii) individual shelter use and (iii) proportion of individual time in shelter with partner between trial one and trial two, controlling for sex, $n=72$ stickleback fish. Dyad ID was included as a random effect. Statistically significant results are shown in bold.

\begin{tabular}{|c|c|c|c|}
\hline \multirow{4}{*}{$\begin{array}{l}\text { Model } \\
\text { LMM1 } \\
\text { Total distance } \\
\text { travelled Trial } 2 \\
\text { Fixed Effects }\end{array}$} & Predictor Variable & Estimate \pm s.e. & p-value \\
\hline & & & \\
\hline & $\begin{array}{l}\text { Intercept } \\
\text { Distance travelled Trial } \\
1\end{array}$ & $\begin{array}{r}-5.21 \pm 4.68 \\
0.35 \pm 0.07\end{array}$ & $<0.001$ \\
\hline & Sex Male & $10.31 \pm 3.38$ & 0.004 \\
\hline Random Effects & Dyad ID & $\begin{array}{l}\chi^{2} \\
12.99\end{array}$ & $\begin{array}{c}p \text {-value } \\
<0.001\end{array}$ \\
\hline $\begin{array}{l}\text { LMM2 } \\
\text { Individual } \\
\text { Shelter use Trial } 2 \\
\text { Fixed Effects }\end{array}$ & $\begin{array}{l}\text { Intercept } \\
\text { Shelter use Trial } 1 \\
\text { Sex Male }\end{array}$ & $\begin{array}{r}18.12 \pm 1.68 \\
0.38 \pm 0.17 \\
-6.94 \pm 2.33\end{array}$ & $\begin{array}{l}0.032 \\
0.004\end{array}$ \\
\hline Random Effects & Dyad ID & $\begin{array}{l}\chi^{2} \\
15.64\end{array}$ & $\begin{array}{l}\boldsymbol{p} \text {-value } \\
0.856\end{array}$ \\
\hline $\begin{array}{l}\text { GLMM1 } \\
\text { \% Together in } \\
\text { Shelter Trial } 2 \\
\text { Fixed Effects }\end{array}$ & $\begin{array}{l}\text { Intercept } \\
\text { \% Together Trial } 1 \\
\text { Sex Male }\end{array}$ & $\begin{array}{l}0.25 \pm 0.05 \\
0.29 \pm 0.15 \\
0.07 \pm 0.08\end{array}$ & $\begin{array}{l}\mathbf{0 . 0 4 9} \\
0.373\end{array}$ \\
\hline Random Effects & Dyad ID & $\begin{array}{l}\chi^{2} \\
73.84\end{array}$ & $\begin{array}{c}p \text {-value } \\
<0.001\end{array}$ \\
\hline
\end{tabular}

Table 8.5: Repeatability of inter-dyad distance travelled between trial one and trial two, $n=36$ stickleback fish dyads.

\begin{tabular}{llll}
\hline Model & Predictor Variable & Estimate \pm s.e. & p-value \\
\hline LM1 & & & \\
Inter-individual distance & & & \\
Trial 2 & & & \\
Fixed Effects & Intercept & $0.36 \pm 0.11$ & \\
& Inter-dyad distance Trial 1 & $0.08 \pm 0.06$ & 0.169 \\
\hline
\end{tabular}


Table 8.6: Factors affecting total distance travelled (m), individual shelter time (mins), proportion of individual time in shelter with partner $(\%)$ and inter-dyad distance $(\mathrm{m})$ across two behaviour trial experiments ( $n=72$ individuals; $n=36$ dyads). Fish ID and Dyad ID was included as random effects where appropriate. Statistically significant results are shown in bold.

\begin{tabular}{|c|c|c|c|}
\hline Model & Predictor Variable & Estimate \pm s.e. & p-value \\
\hline \multicolumn{4}{|l|}{ LMM3 } \\
\hline \multicolumn{4}{|l|}{ Distance travelled } \\
\hline \multirow[t]{6}{*}{ Fixed Effects } & Intercept & $59.95 \pm 9.46$ & \\
\hline & Trial two & $-42.34 \pm 2.77$ & $<0.001$ \\
\hline & Housed socially & $-1.85 \pm 7.58$ & 0.809 \\
\hline & Unfamiliar dyads & $1.51 \pm 7.55$ & 0.843 \\
\hline & Log(Average cortisol) & $-5.65 \pm 2.49$ & 0.025 \\
\hline & Sex Male & $11.52 \pm 4.28$ & 0.008 \\
\hline \multirow[t]{3}{*}{ Random Effects } & & $\chi^{2}$ & p-value \\
\hline & Fish ID & 14.96 & $<0.001$ \\
\hline & Dyad ID & 36.65 & $<0.001$ \\
\hline \multicolumn{4}{|l|}{ LMM4 } \\
\hline \multicolumn{4}{|l|}{ Individual shelter use } \\
\hline \multirow[t]{6}{*}{ Fixed Effects } & Intercept & $6.02 \pm 2.55$ & \\
\hline & Trial two & $12.28 \pm 1.14$ & $<0.001$ \\
\hline & Housed socially & $1.35 \pm 1.99$ & 0.500 \\
\hline & Unfamiliar dyads & $0.83 \pm 1.97$ & 0.674 \\
\hline & Log(Average cortisol) & $0.53 \pm 0.98$ & 0.593 \\
\hline & Sex Male & $-4.93 \pm 1.74$ & 0.006 \\
\hline \multirow[t]{3}{*}{ Random Effects } & & $\chi^{2}$ & p-value \\
\hline & Fish ID & 2.97 & 0.085 \\
\hline & Group ID & 3.69 & 0.055 \\
\hline \multicolumn{4}{|l|}{$\begin{array}{l}\text { GLMM2 } \\
\text { \%Together in shelter }\end{array}$} \\
\hline \multirow[t]{8}{*}{ Fixed Effects } & Intercept & $0.08 \pm 0.80$ & \\
\hline & Trial two & $-1.54 \pm 1.09$ & 0.157 \\
\hline & Housed socially & $-0.24 \pm 0.66$ & 0.713 \\
\hline & Unfamiliar dyads & $-0.80 \pm 0.65$ & 0.219 \\
\hline & Sex Male & $0.94 \pm 0.51$ & 0.069 \\
\hline & Log(Average cortisol) & $-0.08 \pm 0.26$ & 0.744 \\
\hline & Trial two:housed socially & $1.36 \pm 0.90$ & 0.133 \\
\hline & Trial two:unfamiliar dyads & $1.90 \pm 0.91$ & 0.036 \\
\hline
\end{tabular}

LMM5

Inter-dyad distance

Fixed Effects

Intercept

$0.16 \pm 0.03$

Trial two

$0.06 \pm 0.02$

0.002

Housed socially

$-0.01 \pm 0.02$

0.637

Unfamiliar dyads

$-0.01 \pm 0.02$

0.774

\section{Random Effects}




\section{Appendix 3: Supplementary material for Chapter 5}

Questionnaire 8.1: Copy of Perceived Stress Scale adapted for daily use (Cohen et al. 1983).

\section{Perceived Stress Scale}

Participant ID:

Date of Birth:

Gender (Circle): $\quad$ M $\quad$ F
Have you eaten\drank in the last hour? (Circle):

Have you consumed alcohol in the last 12 hours? (Circle):

Have you smoked in the last 12 hours? (Circle):
Yes No

Yes No

Yes No

The questions in this scale ask you about your feelings and thoughts today. In each case, you will be asked to indicate by circling how much you feel or think a certain way.

$0=$ Not at all1 $=$ A little $\quad 2=$ Moderately $\quad 3$ = Quite a Bit $\quad 4$ = Extremely

1. Currently, how upset do you feel because of something that happened unexpectedly today?

2. Currently, do you feel that you are unable to control the important things in your life?

3. Currently, how nervous and stressed do you feel?

$\begin{array}{lllll}0 & 1 & 2 & 3 & 4\end{array}$

4. How confident do you currently feel about your ability to handle your personal problems?

5. Currently, do you feel that things are going your way?

6. Currently, do you feel that you cannot cope with all the things you have to do?

7. Currently, do you feel you are able to control irritations in your life?

8. Currently, how on top of things do you feel?

9. Currently, how angered are you because of things that are outside of your control?

10. Currently, do you feel difficulties are piling up so high that you cannot overcome them? 
Questionnaire 8.2: Copy of International Personality Item Pool (IPIP), to determine Big5 personality traits, given to each participant to complete.

Participant ID:

Date of Birth

Gender (Circle): M F

\section{How Accurately Can You Describe Yourself?}

Describe yourself as you generally are now, not as you wish to be in the future. Describe yourself as you honestly see yourself, in relation to other people you know of the same sex as you are, and roughly your same age. So that you can describe yourself in an honest manner, your responses will be kept in absolute confidence. Indicate for each statement whether it is 1. Very Inaccurate, 2. Moderately Inaccurate, 3. Neither Accurate Nor Inaccurate, 4. Moderately Accurate, or 5. Very Accurate as a description of you.

1. Am the life of the party.

\begin{tabular}{lllll} 
Very & \multicolumn{3}{c}{$\begin{array}{l}\text { Neither } \\
\text { Accurate }\end{array}$} \\
Inaccurate & $\begin{array}{l}\text { Moderately } \\
\text { Inaccurate }\end{array}$ & $\begin{array}{l}\text { Nor } \\
\text { Inaccurate }\end{array}$ & $\begin{array}{l}\text { Moderately } \\
\text { Accurate }\end{array}$ & $\begin{array}{l}\text { Very } \\
\text { Accurate }\end{array}$ \\
0 & 0 & 0 & 0 & 0 \\
0 & 0 & 0 & 0 & 0 \\
0 & 0 & 0 & 0 & 0 \\
0 & 0 & 0 & 0 & 0 \\
0 & 0 & 0 & 0 & 0 \\
0 & 0 & 0 & 0 & 0 \\
0 & 0 & 0 & 0 & 0 \\
0 & 0 & 0 & 0 & 0
\end{tabular}

2. Feel little concern for others.

3. Am always prepared.

4. Get stressed out easily.

5. Have a rich vocabulary.

6. Don't talk a lot.

7. Am interested in people.

8. Leave my belongings around.

9. Am relaxed most of the time.

0

10. Have difficulty understanding abstract ideas.

0

0

0

11. Feel comfortable around people.

12. Insult people.

13. Pay attention to details.

14. Worry about things.

15. Have a vivid imagination. $O$

16. Keep in the background.

17. Sympathize with others' feelings.

18. Make a mess of things. $O$

0

19. Seldom feel blue.

$\mathrm{O}$

20. Am not interested in abstract ideas.

0

21. Start conversations.

O

0

0
O

0

0

0

$\mathrm{O}$

0

O

0

0

0

0

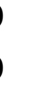

0
0
0
0

0

0

0

0

O

0

0

0

0
0

\section{$(4+)$}




$\begin{array}{lllll}\text { Very } & \text { Moderately } & \text { Neither } & \text { Moderately } & \text { Very } \\ \text { Inaccurate } & \text { Inaccurate } & \begin{array}{l}\text { Accurate } \\ \text { Nor } \\ \text { Inaccurate }\end{array} & \text { Accurate } & \text { Accurate } \\ & & \end{array}$

22. Am not interested in other people's problems.

23. Get chores done right away.

0

24. Am easily disturbed.

25. Have excellent ideas.

26. Have little to say.

27. Have a soft heart.

28. Often forget to put things back in their proper place.

29. Get upset easily.

0

0

O

0

30. Do not have a good imagination.

31. Talk to a lot of different people at parties.

32. Am not really interested in others.

33. Like order.

34. Change my mood a lot.

35. Am quick to understand things.

36. Don't like to draw

attention to myself.

37. Take time out for others. 0

38. Shirk my duties.

39. Have frequent mood

swings.

40. Use difficult words.

41. Don't mind being the centre of attention.

0

$\mathrm{O}$

0

0

0

0

$0 \quad 0$

0

0

0

0

0

0

O

0

O

0

0

42. Feel others' emotions. $O$

0

0

43. Follow a schedule.

0

44. Get irritated easily.

0

0

0

45. Spend time reflecting on things.

0

0

46. Am quiet around strangers.

0

0

47. Make people feel at ease. $O$

0

48. Am exacting in my work. $O$

0

49. Often feel blue.

0

50. Am full of ideas.

0

o 
Table 8.7: Complete table with all measures (pre- and post-hormone concentrations, PSS and scores for each personality trait) for each participant, $\mathrm{n}=13$.

\begin{tabular}{|c|c|c|c|c|c|c|c|c|c|c|c|c|c|}
\hline ID & Sex & Age & $\begin{array}{l}\text { Pre-Cortisol } \\
\text { concentration } \\
(\mu \mathrm{g} / \mathrm{dL})\end{array}$ & $\begin{array}{l}\text { Post-Cortisol } \\
\text { concentration } \\
(\mu \mathrm{g} / \mathrm{dL})\end{array}$ & $\begin{array}{l}\text { Pre- } \\
\text { Testosterone } \\
\text { concentration } \\
(\mathrm{pg} / \mathrm{ml})\end{array}$ & $\begin{array}{l}\text { Post- } \\
\text { Testosterone } \\
\text { concentration } \\
(\mathrm{pg} / \mathrm{ml})\end{array}$ & $\begin{array}{l}\text { Pre- } \\
\text { PSS }\end{array}$ & $\begin{array}{l}\text { Post- } \\
\text { PSS }\end{array}$ & Openness & Conscientiousness & Extraversion & Agreeableness & Neuroticism \\
\hline P1 & $\mathrm{M}$ & 28 & 0.118 & 0.103 & 180.313 & 156.887 & 13 & 16 & 30 & 30 & 30 & 30 & 30 \\
\hline P2 & $\mathrm{F}$ & 42 & 0.132 & 0.105 & 64.039 & 62.399 & 22 & 11 & 41 & 34 & 30 & 40 & 24 \\
\hline P3 & $M$ & 35 & 0.148 & 0.092 & 181.272 & 149.626 & 8 & 5 & 47 & 32 & 29 & 42 & 38 \\
\hline P4 & $M$ & 37 & 0.097 & 0.076 & 197.792 & 189.302 & 13 & 7 & 39 & 26 & 34 & 40 & 27 \\
\hline P5 & $M$ & 57 & 0.094 & 0.200 & 144.037 & 143.050 & 11 & 11 & 31 & 26 & 32 & 43 & 35 \\
\hline P6 & $\mathrm{F}$ & 24 & 0.185 & 0.112 & 135.260 & 88.608 & 17 & 11 & 44 & 32 & 41 & 45 & 25 \\
\hline P7 & $\mathrm{F}$ & 23 & 0.115 & 0.174 & 105.681 & 126.038 & 24 & 17 & 43 & 40 & 26 & 48 & 21 \\
\hline P8 & $M$ & 27 & 0.582 & 0.285 & 103.307 & 93.417 & 6 & 3 & 48 & 35 & 26 & 37 & 32 \\
\hline P9 & $M$ & 25 & 0.209 & 0.109 & 176.463 & 147.003 & 15 & 19 & 49 & 35 & 37 & 47 & 34 \\
\hline P10 & $M$ & 42 & 0.212 & 0.142 & 161.005 & 240.326 & 8 & 6 & 43 & 43 & 35 & 41 & 39 \\
\hline P11 & $\mathrm{F}$ & 54 & 0.190 & 0.120 & 109.035 & 87.616 & 16 & 17 & 45 & 36 & 26 & 31 & 18 \\
\hline P12 & $\mathrm{F}$ & 46 & 0.283 & 0.237 & 64.908 & 69.553 & 4 & 5 & 45 & 38 & 37 & 42 & 46 \\
\hline P13 & $\mathrm{M}$ & 43 & 0.070 & 0.060 & 124.165 & 120.259 & 19 & 12 & 46 & 19 & 44 & 46 & 33 \\
\hline
\end{tabular}


Table 8.8: Additional data set, including two cortisol concentrations, PSS, sex and age of each participant, $\mathrm{n}=56$.

\begin{tabular}{|c|c|c|c|c|c|}
\hline ID & Sex & Age & Cortisol Concentration $(A)(\mu \mathrm{g} / \mathrm{dL})$ & Cortisol Concentration $(B)(\mu \mathrm{g} / \mathrm{dL})$ & PSS \\
\hline 6 & $\mathrm{~F}$ & 21 & 0.892 & 0.405 & 10 \\
\hline 9 & $\mathrm{~F}$ & 20 & 1.072 & 0.733 & 17 \\
\hline 10 & $\mathrm{~F}$ & 22 & 0.513 & 0.282 & 17 \\
\hline 17 & $\mathrm{~F}$ & 22 & 0.829 & 0.764 & 26 \\
\hline 18 & $\mathrm{~F}$ & 23 & 0.507 & 0.242 & 12 \\
\hline 19 & $\mathrm{~F}$ & 21 & 0.45 & 0.669 & 8 \\
\hline 20 & $\mathrm{~F}$ & 26 & 0.48 & 0.169 & 7 \\
\hline 23 & $\mathrm{~F}$ & 22 & 1 & 0.696 & 15 \\
\hline 24 & $\mathrm{~F}$ & 21 & 0.31 & 0.305 & 14 \\
\hline 25 & $\mathrm{~F}$ & 21 & 0.541 & 0.359 & 20 \\
\hline 26 & $\mathrm{~F}$ & 20 & 0.273 & 0.16 & 13 \\
\hline 27 & $\mathrm{~F}$ & 22 & 0.515 & 0.295 & 15 \\
\hline 28 & $\mathrm{~F}$ & 22 & 0.473 & 0.367 & 12 \\
\hline 29 & $\mathrm{~F}$ & 20 & 0.42 & 0.192 & 9 \\
\hline 30 & $\mathrm{~F}$ & 20 & 0.651 & 0.329 & 21 \\
\hline 33 & $\mathrm{~F}$ & 20 & 0.786 & 0.573 & 18 \\
\hline 34 & $F$ & 21 & 0.238 & 0.222 & 11 \\
\hline 35 & $\mathrm{~F}$ & 23 & 0.526 & 0.552 & 21 \\
\hline 37 & $\mathrm{~F}$ & 22 & 0.797 & 0.679 & 17 \\
\hline 38 & $\mathrm{~F}$ & 21 & 0.632 & 0.388 & 5 \\
\hline 39 & $\mathrm{~F}$ & 20 & 0.451 & 0.334 & 17 \\
\hline 42 & $\mathrm{~F}$ & 20 & 0.41 & 0.219 & 14 \\
\hline 43 & $\mathrm{~F}$ & 21 & 0.398 & 0.232 & 19 \\
\hline 44 & $\mathrm{~F}$ & 21 & 0.545 & 0.29 & 8 \\
\hline 45 & $\mathrm{~F}$ & 22 & 0.452 & 0.296 & 29 \\
\hline 47 & $F$ & 20 & 0.54 & 0.492 & 9 \\
\hline 48 & $F$ & 21 & 0.429 & 0.194 & 15 \\
\hline 49 & $F$ & 22 & 0.41 & 0.231 & 17 \\
\hline 50 & $\mathrm{~F}$ & 21 & 1.553 & 0.898 & 9 \\
\hline 51 & $F$ & 20 & 0.931 & 0.546 & 25 \\
\hline 52 & $F$ & 21 & 0.961 & 0.543 & 25 \\
\hline 55 & $\mathrm{~F}$ & 20 & 0.468 & 0.3 & 16 \\
\hline 1 & $M$ & 23 & 0.307 & 0.253 & 26 \\
\hline 2 & $M$ & 23 & 0.594 & 0.231 & 16 \\
\hline 3 & $M$ & 20 & 0.309 & 0.158 & 16 \\
\hline 4 & $\mathrm{M}$ & 23 & 0.413 & 0.218 & 14 \\
\hline 5 & $M$ & 22 & 0.743 & 0.339 & 16 \\
\hline 7 & $M$ & 28 & 0.12 & 0.09 & 40 \\
\hline 8 & $M$ & 21 & 0.814 & 0.549 & 23 \\
\hline 11 & $M$ & 21 & 0.869 & 0.592 & 11 \\
\hline 12 & $M$ & 22 & 1.027 & 0.493 & 15 \\
\hline 13 & $\mathrm{M}$ & 21 & 1.193 & 0.619 & 13 \\
\hline
\end{tabular}




\begin{tabular}{rrrrrr}
\hline ID & Sex & Age & Cortisol Concentration $(A)(\mu \mathrm{g} / \mathrm{dL})$ & Cortisol Concentration $(B)(\mu \mathrm{g} / \mathrm{dL})$ & PSS \\
\hline 14 & $\mathrm{M}$ & 20 & 0.198 & 0.132 & 17 \\
15 & $\mathrm{M}$ & 21 & 0.17 & 0.131 & 8 \\
21 & $\mathrm{M}$ & 22 & 0.55 & 0.38 & 25 \\
22 & $\mathrm{M}$ & 22 & 1.003 & 0.401 & 17 \\
31 & $\mathrm{M}$ & 23 & 0.112 & 0.154 & 5 \\
32 & $\mathrm{M}$ & 21 & 0.581 & 0.592 & 13 \\
36 & $\mathrm{M}$ & 22 & 0.524 & 0.3 & 14 \\
40 & $\mathrm{M}$ & 21 & 1.22 & 0.625 & 9 \\
41 & $\mathrm{M}$ & 21 & 0.869 & 0.544 & 12 \\
46 & $\mathrm{M}$ & 21 & 0.403 & 0.257 & 8 \\
53 & $\mathrm{M}$ & 21 & 0.856 & 0.421 & 7 \\
54 & $\mathrm{M}$ & 21 & 1.171 & 0.499 & 10 \\
56 & $\mathrm{M}$ & 21 & 0.262 & 0.107 & 7 \\
\hline
\end{tabular}




\section{Appendix 4: ASAB Conference Poster}

Poster incorporating results from Chapter 4; presented at ASAB Summer Conference 2019, New Frontiers in the Study of Animal Behaviour in Konstanz, Germany.

\section{The effects of familiarity on repeatability of behaviour in pairs of three-spined sticklebacks}

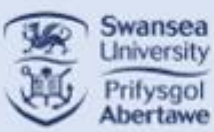

Amanda Fry, Andrew King, Ines Fürtbauer

Department of Biosciences, College of Science, Swansea University, Swansea, UK

amanda.fry@swansea.ac.uk@ eAmandaFry 270

\section{HYPOTHESIS AND PREDICTIONS}

Across animal species, individuals prefer to associate with familiar partners ${ }^{1,2}$ and familiarity between individuals can affect behaviour ${ }^{3}$, altering the type ${ }^{4}$ and repeatability of behaviour ${ }^{3}$, as well as the dynamics of social interactions ${ }^{6}$.

We hypothesised that familiarity would affect the repeatability and type of behaviours shown by pairs of three-spined stickleback fish, and predicted familiar pairs would show 1) stronger social attraction, 2) more active behaviours, and 3) greater repeatability compared to unfamiliar fish.

\section{METHODS}

$\mathrm{N}=48$ fish ( $\mathrm{n}=12$ familiar dyads and $\mathrm{n}=12$ unfamiliar dyads) were filmed in a novel environment containing two shelters ( $n=2$ trials). Using image-tracking analyses, the (i) distance travelled $(\mathrm{m})$, (ii) individual shelter use (mins), (iii) shared shelter use (\%), and (iv) pair inter-individual distance ( $\mathrm{m}$ ) were extracted for each fish in each trial.

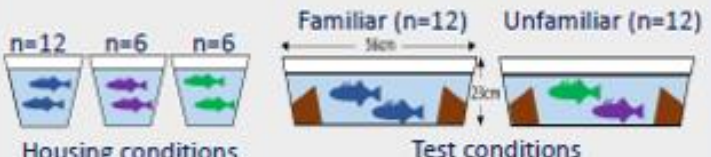

\section{RESULTS}
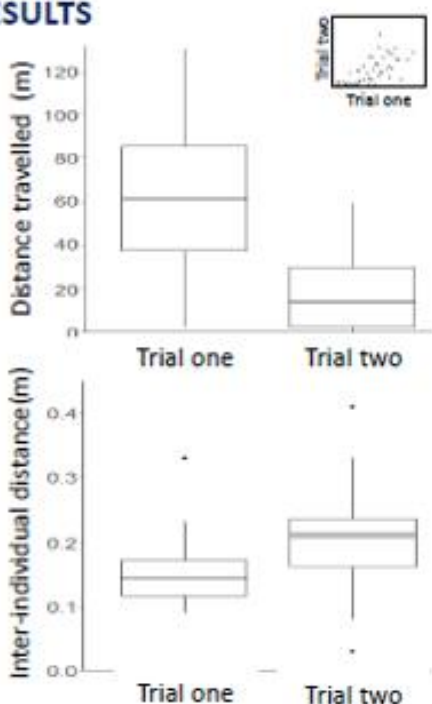

Trial one Trial two

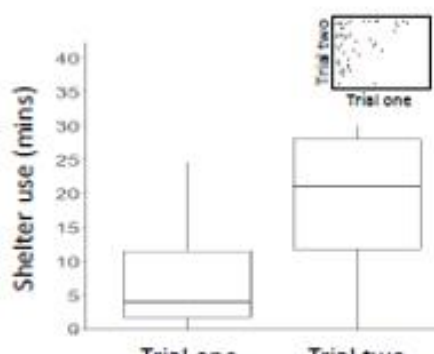

Trial one

Trial two

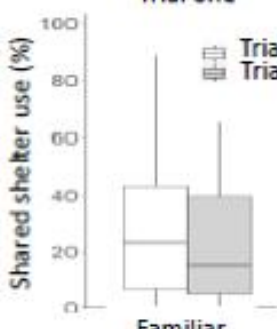

Familiar

Familiarity did not affect distance travelled, sheiter use, or interindividual distance (LMMs: $p>0.05$ )

Distance travelled was repeatable across trials (LMM : p<0.001) and lower in trial 2 (LMM: $p<0.001$ )

Shelter use was repeatable across trials (LMM: $p=0.019)$, and greater in trial 2 (LMM: $p<0.001$ )

Inter-individual distance was not repeatable ( $L M M$ : $p=0.593$ ) and greater in trial 2 (LMM: $p=0.014)$

Shared sheiter use was not repeatable (GLMM: $p=0.169$ ) and was higher in trial 2 for unfamiliar dyads (GLMM: $p=0.009$ )

\section{DISCUSSION}

Contrary to our hypothesis and predictions, familiarity did not affect fish behaviour in three of four of our behavioural measures. Instead, the current social and ecological environment (trial) appear to explain variation across our dataset. The lack of a major familiarity effect could be explained by the length of time fish spend together and/or the short-term nature of our testing. Further investigations will be required to test these ideas.

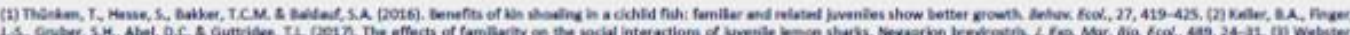

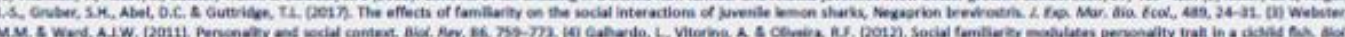

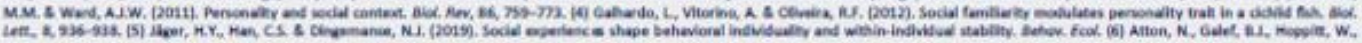

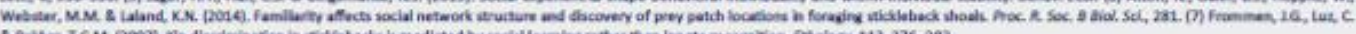

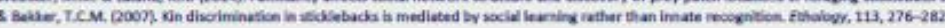




\section{Appendix 5: ECBB Conference Poster}

Poster incorporating results from Chapter 6; presented at ECBB Summer Conference 2018, $9^{\text {th }}$ European Conference in Behavioural Biology in Liverpool, UK.

\section{Sheep wool cortisol: Intra-individual repeatability and plasticity in response to grazing-related changes in body mass \\ Ines Fürtbauer ${ }^{1}$, Charlotte Solman ${ }^{1}$, Amanda Fry ${ }^{1}$ \\ ${ }^{1}$ Department of Biosciences, Swansea University, UK

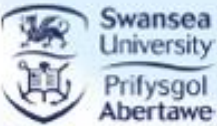

Repeatability of wool cortisol concentrations (WCC) and relationships with body weight:
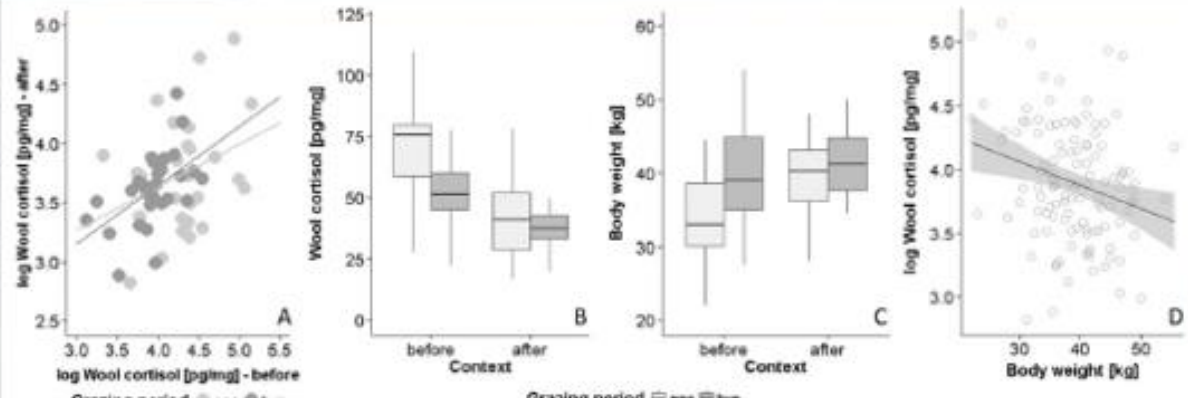

(A) Repeatability in WCC (LMM: $p=0.003$ ). Differences between (B) WOC (LMM: $p<0.001$ ) and (C) body weight (LMM: $p<0.001$ ) before and after two one-month long grazing periods. (D) Relationship between body weight and WCC (LMM: $\mathrm{p}=0.009$ )

Methods:

- $\mathrm{N}=33$ barren ewes

$\cdot N=150$ wool samples

* $\mathrm{N}=2$ one-month grazing periods in semi-improved enclosed 11.5 ha upland pasture at Bangor University's Henfaes Research Centre, North Wales; sheep weighed before and after

- WCC assessed via enzyme immunoassay

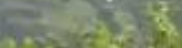

ancis

Conclusions:

- Consistent individual differences in WCC

- Potential use of WCC as a measure of

physical condition/nutritional stress

- Differences in WCC across the wool shaft

may reflect differences in hypothalamic-

pituitary-adrenal (HPA) axis activity during

different time windows ('cortisol calendar')

- Use of WCC to study effects of gradually

changing variables on long-term HPA axis

activity

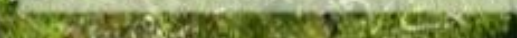

sề, i.fuertbauer@swansea.ac.uk (सil) http://ines.fuertbauer.com Y@ines_fuertbauer
Pürtbauer L., Solman C, \&. Fry A. (under review) Wooi cortisol as a retrospective measure of long-term physiological stress
Acknowledgements:

Andrew King, Lucy Lush, oplems,o Uplands $\mathrm{N}_{2} \mathrm{O}$ project

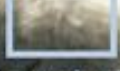

Lower back samples (n=120)

WCC across the wool shaft:

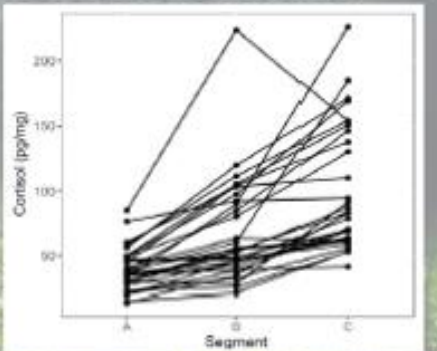

Significant differences in wcc between segments (LMM: $p<0.001$ ) \& consistent individual differences random effect iD; $p<0.001$ )

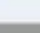

a




\section{Chapter 9: References}

Abouheif, M., Al-Owaimer, A., Kraidees, M., Metwally, H. \& Shafey, T. (2013). Effect of restricted feeding and realimentation on feed performance and carcass characteristics of growing lambs. Rev. Bras. Zootec., 42, 95-101.

Accorsi, P.A., Carloni, E., Valsecchi, P., Viggiani, R., Gamberoni, M., Tamanini, C., et al. (2008). Cortisol determination in hair and faeces from domestic cats and dogs. Gen. Comp. Endocrinol., 155, 398-402.

Adam, E.K. \& Kumari, M. (2009). Assessing salivary cortisol in large-scale, epidemiological research. Psychoneuroendocrinology, 34, 1423-1436.

Adams, N.J., Cockrem, J.F., Taylor, G.A., Candy, E.J. \& Bridges, J. (2005). Corticosterone responses of hand-reared and parent-reared grey-faced petrel chicks (Pterodroma macroptera gouldi). Zoo Biol., 24, 283-290.

Adams, N.J., Farnworth, M.J., Rickett, J., Parker, K.A. \& Cockrem, J.F. (2011). Behavioural and corticosterone responses to capture and confinement of wild blackbirds (Turdus merula). Appl. Anim. Behav. Sci., 134, 246-255.

Adkins-Regan, E. (2005). Hormones and animal social behavior. Princeton University Press.

Afrisham, R., Sadegh-Nejadi, S., SoliemaniFar, O., Kooti, W., Ashtary-Larky, D., Alamiri, F., et al. (2016). Salivary testosterone levels under psychological stress and its relationship with rumination and five personality traits in medical students. Psychiatry Investig., 13, 637.

Agrawal, A.A. (2001). Ecology: Phenotypic plasticity in the interactions and evolution of species. Science (80-. ).

Allen, A.P., Kennedy, P.J., Cryan, J.F., Dinan, T.G. \& Clarke, G. (2014). Biological and psychological markers of stress in humans: Focus on the Trier Social Stress Test. Neurosci. Biobehav. Rev.

Alsop, D. \& Vijayan, M. (2009). The zebrafish stress axis: Molecular fallout from the teleost-specific genome duplication event. Gen. Comp. Endocrinol., 161, 62-66.

Altmann, J., Lynch, J.W., Nguyen, N., Alberts, S.C. \& Gesquiere, L.R. (2004). Lifehistory correlates of steroid concentrations in wild peripartum baboons. Am. J. Primatol., 64, 95-106.

Anestis, S.F., Bribiescas, R.G. \& Hasselschwert, D.L. (2006). Age, rank, and personality effects on the cortisol sedation stress response in young chimpanzees. Physiol. Behav., 89, 287-294.

Angelier, F., Weimerskirch, H., Dano, S. \& Chastel, O. (2007). Age, experience and reproductive performance in a long-lived bird: A hormonal perspective. Behav. Ecol. Sociobiol., 61, 611-621.

Archard, G.A., Earley, R.L., Hanninen, A.F. \& Braithwaite, V.A. (2012). Correlated behaviour and stress physiology in fish exposed to different levels of predation pressure. Funct. Ecol., 26, 637-645. 
Ash, H., Smith, T.E., Knight, S. \& Buchanan-Smith, H.M. (2018). Measuring physiological stress in the common marmoset (Callithrix jacchus): Validation of a salivary cortisol collection and assay technique. Physiol. Behav., 185, 14-22.

Ashley, P.J. (2007). Fish welfare: Current issues in aquaculture. Appl. Anim. Behav. Sci., 104, 199-235.

Atton, N., Galef, B.J., Hoppitt, W., Webster, M.M. \& Laland, K.N. (2014). Familiarity affects social network structure and discovery of prey patch locations in foraging stickleback shoals. Proc. R. Soc. B Biol. Sci., 281, 20140579-20140579.

Atwell, J.W., Cardoso, G.C., Whittaker, D.J., Campbell-Nelson, S., Robertson, K.W. \& Ketterson, E.D. (2012). Boldness behavior and stress physiology in a novel urban environment suggest rapid correlated evolutionary adaptation. Behav. Ecol., 23, 960-969.

Auer, S.K., Salin, K., Rudolf, A.M., Anderson, G.J. \& Metcalfe, N.B. (2015). Flexibility in metabolic rate confers a growth advantage under changing food availability. J. Anim. Ecol., 84, 1405-1411.

Baile, C.A. \& Forbes, J.M. (1974). Control of feed intake and regulation of energy balance in ruminants. Physiol. Rev., 54, 160-214.

Banerjee, S.B. \& Adkins-Regan, E. (2011). Effect of isolation and conspecific presence in a novel environment on corticosterone concentrations in a social avian species, the zebra finch (Taeniopygia guttata). Horm. Behav., 60, 233-238.

Barnett, A.G. (2004). Regression to the mean: what it is and how to deal with it. Int. J. Epidemiol., 34, 215-220.

Bartolomucci, A., Palanza, P., Sacerdote, P., Ceresini, G., Chirieleison, A., Panerai, A.., et al. (2003). Individual housing induces altered immuno-endocrine responses to psychological stress in male mice. Psychoneuroendocrinology, 28, $540-558$.

Barton, B.A. (2002). Stress in Fishes: A Diversity of Responses with Particular Reference to Changes in Circulating Corticosteroids. Integr. Comp. Biol., 42, $517-525$.

Basarab, J.A., Price, M.A., Aalhus, J.L., Okine, E.K., Snelling, W.M. \& Lyle, K.L. (2003). Residual feed intake and body composition in young growing cattle. Can. J. Anim. Sci., 83, 189-204.

Batabyal, A. \& Thaker, M. (2019). Social coping styles of lizards are reactive and not proactive in urban areas. Gen. Comp. Endocrinol., 270, 67-74.

Bates, D., Mächler, M., Bolker, B. \& Walker, S. (2014). Fitting linear mixed-effects models using lme4.

Bates, D.M. \& Pinheiro, J.C. (2000). Mixcd-Effects Models in S and S-PLUS. New York Springer-Verlag.

Bateup, H.S., Booth, A., Shirtcliff, E.A. \& Granger, D.A. (2002). Testosterone, cortisol, and women's competition. Evol. Hum. Behav., 23, 181-192.

Baugh, A.T., Bastien, B., Still, M.B. \& Stowell, N. (2018). Validation of water-borne 
steroid hormones in a tropical frog (Physalaemus pustulosus). Gen. Comp. Endocrinol., 261, 67-80.

Baugh, A.T., Schaper, S. V., Hau, M., Cockrem, J.F., de Goede, P. \& Oers, K. van. (2012). Corticosterone responses differ between lines of great tits (Parus major) selected for divergent personalities. Gen. Comp. Endocrinol., 175, 488-494.

Bedgood, D., Boggiano, M.M. \& Turan, B. (2014). Testosterone and social evaluative stress: the moderating role of basal cortisol. Psychoneuroendocrinology, 47, 107115 .

Behie, A.M., Pavelka, M.S.M. \& Chapman, C.A. (2010). Sources of variation in fecal cortisol levels in howler monkeys in belize. Am. J. Primatol., 72, n/a-n/a.

Behringer, V. \& Deschner, T. (2017). Non-invasive monitoring of physiological markers in primates. Horm. Behav.

Bell, A.M., Hankison, S.J. \& Laskowski, K.L. (2009). The repeatability of behaviour: a meta-analysis. Anim. Behav., 77, 771-783.

Bell, A.M., Henderson, L. \& Huntingford, F.A. (2010). Behavioral and respiratory responses to stressors in multiple populations of three-spined sticklebacks that differ in predation pressure. J. Comp. Physiol. B, 180, 211-220.

Bennett, A. \& Hayssen, V. (2010). Measuring cortisol in hair and saliva from dogs: Coat color and pigment differences. Domest. Anim. Endocrinol., 39, 171-180.

Bensky, M.K., Paitz, R., Pereira, L. \& Bell, A.M. (2017). Testing the predictions of coping styles theory in threespined sticklebacks. Behav. Processes, 136, 1-10.

Bergamasco, L., Osella, M.C., Savarino, P., Larosa, G., Ozella, L., Manassero, M., et al. (2010). Heart rate variability and saliva cortisol assessment in shelter dog: Human-animal interaction effects. Appl. Anim. Behav. Sci., 125, 56-68.

Bibbey, A., Carroll, D., Roseboom, T.J. \& de Rooij, S.R. (2013). Personality and physiological reactions to acute psychological stress. Int. J. Psychophysiol., 90, $28-36$.

Bierbach, D., Laskowski, K.L. \& Wolf, M. (2017). Behavioural individuality in clonal fish arises despite near-identical rearing conditions. Nat. Commun., 8, 1-7.

Bines, J.A. \& Morant, S. V. (1983). The effect of body condition on metabolic changes associated with intake of food by the cow. Br. J. Nutr., 50, 81-89.

Blas, J., Bortolotti, G.R., Tella, J.L., Baos, R. \& Marchant, T.A. (2007). Stress response during development predicts fitness in a wild, long lived vertebrate. Proc. Natl. Acad. Sci. U. S. A., 104, 8880-8884.

Blaxter, K.L., Wainman, F.W. \& Wilson, R.S. (1961). The regulation of food intake by sheep. Anim. Prod., 3, 51-61.

Blom, S., Andersson, T.B. \& Förlin, L. (2000). Effects of food deprivation and handling stress on head kidney 17 $\alpha$-hydroxyprogesterone 21-hydroxylase activity, plasma cortisol and the activities of liver detoxification enzymes in rainbow trout. Aquat. Toxicol., 48, 265-274.

Blumstein, D. (2012). Social behaviour. In: Behavioural Responses to a Changing 
World: Mechanisms and Consequences (eds. Candolin, U. \& Wong, B.B.M.). Oxford University Press, Oxford, pp. 119-128.

Bolivar, V.J. (2009). Intrasession and intersession habituation in mice: From inbred strain variability to linkage analysis. Neurobiol. Learn. Mem., 92, 206-214.

Bonier, F. \& Cox, R.M. (2020). Do hormone manipulations reduce fitness? A metaanalytic test of the Optimal Endocrine Phenotype Hypothesis. Mol. Cell. Endocrinol.

Bonier, F., Martin, P.R., Moore, I.T. \& Wingfield, J.C. (2009). Do baseline glucocorticoids predict fitness? Trends Ecol. Evol.

Brelin, D., Petersson, E. \& Winberg, S. (2005). Divergent Stress Coping Styles in Juvenile Brown Trout ( Salmo trutta ). Ann. N. Y. Acad. Sci., 1040, 239-245.

Brosnan, S.F. \& Postma, E. (2017). Humans as a model for understanding biological fundamentals. Proc. R. Soc. B Biol. Sci., 284, 20172146.

Brown, W.A., Monti, P.M. \& Corriveau, D.P. (1978). Serum testosterone and sexual activity and interest in men. Arch. Sex. Behav., 7, 97-103.

Buchanan, K.L. \& Partecke, J. (2012). The endocrine system: can homeostasis be maintained in a changing world? In: Behavioural Responses to a Changing World: Mechanisms and Consequences (eds. Candolin, U. \& Wong, B.). Oxford University Press, Oxford, pp. 32-45.

Buck, C.L., O'Reilly, K.M. \& Kildaw, S.D. (2007). Interannual variability of Blacklegged Kittiwake productivity is reflected in baseline plasma corticosterone. Gen. Comp. Endocrinol., 150, 430-436.

Burnard, C., Ralph, C., Hynd, P., Hocking Edwards, J. \& Tilbrook, A. (2017). Hair cortisol and its potential value as a physiological measure of stress response in human and non-human animals. Anim. Prod. Sci., 57, 401.

Buttner, A., Thompson, B., Strasser, R. \& Santo, J. (2015). Evidence for a synchronization of hormonal states between humans and dogs during competition. Physiol. Behav., 142, 54-62.

Campbell, J. \& Ehlert, U. (2012). Acute psychosocial stress: does the emotional stress response correspond with physiological responses? Psychoneuroendocrinology, $37,1111-1134$.

Canale, C. \& Henry, P. (2010). Adaptive phenotypic plasticity and resilience of vertebrates to increasing climatic unpredictability. Clim. Res., 43, 135-147.

Cappeliez, P. \& O'Rourke, N. (2002). Profiles of reminiscence among older adults: Perceived stress, life attitudes, and personality variables. Int. J. Aging Hum. Dev., 54, 255-266.

Carere, C. \& Van Oers, K. (2004). Shy and bold great tits (Parus major): Body temperature and breath rate in response to handling stress. Physiol. Behav., 82, 905-912.

Carlitz, E.H.D., Kirschbaum, C., Miller, R., Rukundo, J. \& van Schaik, C.P. (2015). Effects of body region and time on hair cortisol concentrations in chimpanzees 
(Pan troglodytes). Gen. Comp. Endocrinol., 223, 9-15.

Carlitz, E.H.D., Kirschbaum, C., Stalder, T. \& van Schaik, C.P. (2014). Hair as a longterm retrospective cortisol calendar in orang-utans (Pongo spp.): New perspectives for stress monitoring in captive management and conservation. Gen. Comp. Endocrinol., 195, 151-156.

Carter, A., Goldizen, A. \& Heinsohn, R. (2012). Personality and plasticity: temporal behavioural reaction norms in a lizard, the Namibian rock agama. Anim. Behav., $84,471-477$.

Casal, N., Manteca, X., Peña L, R., Bassols, A. \& Fàbrega, E. (2017). Analysis of cortisol in hair samples as an indicator of stress in pigs. J. Vet. Behav., 19, 1-6.

Caslini, C., Comin, A., Peric, T., Prandi, A., Pedrotti, L. \& Mattiello, S. (2016). Use of hair cortisol analysis for comparing population status in wild red deer (Cervus elaphus) living in areas with different characteristics. Eur. J. Wildl. Res., 62, 713723.

Castanheira, M.F., Conceição, L.E.C., Millot, S., Rey, S., Bégout, M.-L., Damsgård, B., et al. (2017). Coping styles in farmed fish: consequences for aquaculture. Rev. Aquac., 9, 23-41.

Casto, K. V. \& Edwards, D.A. (2016). Before, during, and after: how phases of competition differentially affect testosterone, cortisol, and estradiol levels in women athletes. Adapt. Hum. Behav. Physiol., 2, 11-25.

Casto, K. V, Elliott, C.M. \& Edwards, D.A. (2014). Original research intercollegiate cross country competition: effects of warm-up and racing on salivary levels of cortisol and testosterone. Int. J. Exerc. Sci.

Castro, W.L.R. \& Matt, K.S. (1997). The Importance of Social Condition in the Hormonal and Behavioral Responses to an Acute Social Stressor in the Male Siberian Dwarf Hamster (Phodopus sungorus). Horm. Behav., 32, 209-216.

Chapman, C.A., Schoof, V.A.M., Bonnell, T.R., Gogarten, J.F. \& Calmé, S. (2015). Competing pressures on populations: long-term dynamics of food availability, food quality, disease, stress and animal abundance. Philos. Trans. R. Soc. Lond. B. Biol. Sci., 370, 20140112.

Chivers, D.P., Brown, G.E. \& Smith, R.J.F. (1995). Familiarity and shoal cohesion in fathead minnows ( Pimephales promelas ): implications for antipredator behaviour. Can. J. Zool., 73, 955-960.

Chua, B., Coenen, E., van Delen, J. \& Weary, D.M. (2002). Effects of Pair Versus Individual Housing on the Behavior and Performance of Dairy Calves. J. Dairy Sci., 85, 360-364.

Clements, A.D. (2013). Salivary cortisol measurement in developmental research: Where do we go from here? Dev. Psychobiol., 55, 205-220.

Clinchy, M., Zanette, L., Boonstra, R., Wingfield, J.C. \& Smith, J.N.M. (2004). Balancing food and predator pressure induces chronic stress in songbirds. Proceedings. Biol. Sci., 271, 2473-9.

Cockrem, J.F. (2007). Stress, corticosterone responses and avian personalities. J. 
Ornithol., 148, 169-178.

Cockrem, J.F. (2013a). Corticosterone responses and personality in birds: Individual variation and the ability to cope with environmental changes due to climate change. Gen. Comp. Endocrinol., 190, 156-163.

Cockrem, J.F. (2013b). Individual variation in glucocorticoid stress responses in animals. Gen. Comp. Endocrinol., 181, 45-58.

Cockrem, J.F., Barrett, D.P., Candy, E.J. \& Potter, M.A. (2009). Corticosterone responses in birds: Individual variation and repeatability in Adelie penguins (Pygoscelis adeliae) and other species, and the use of power analysis to determine sample sizes. Gen. Comp. Endocrinol., 163, 158-168.

Cockrem, J.F., Candy, E.J., Barrett, D.P., Agnew, P. \& Potter, M.A. (2017). Individual variation and repeatability of corticosterone responses of little penguins (Eudyptula minor) sampled in two successive years at Oamaru, New Zealand. Gen. Comp. Endocrinol., 244, 86-92.

Cockrem, J.F., Potter, M.A., Barrett, D.P. \& Candy, E.J. (2008). Corticosterone Responses to Capture and Restraint in Emperor and Adelie Penguins in Antarctica. Zoolog. Sci., 25, 291-298.

Cockrem, J.F., Potter, M.A. \& Candy, E.J. (2006). Corticosterone in relation to body mass in Adelie penguins (Pygoscelis adeliae) affected by unusual sea ice conditions at Ross Island, Antarctica. Gen. Comp. Endocrinol., 149, 244-252.

Cohen, S., Kamarck, T. \& Mermelstein, R. (1983). A global measure of perceived stress. J. Health Soc. Behav., 24, 385.

Colson, V., Martin, E., Orgeur, P. \& Prunier, A. (2012). Influence of housing and social changes on growth, behaviour and cortisol in piglets at weaning. Physiol. Behav., 107, 59-64.

Comin, A., Prandi, A., Peric, T., Corazzin, M., Dovier, S. \& Bovolenta, S. (2011). Hair cortisol levels in dairy cows from winter housing to summer highland grazing. Livest. Sci., 138, 69-73.

Comin, A., Veronesi, M.C., Montillo, M., Faustini, M., Valentini, S., Cairoli, F., et al. (2012). Hair cortisol level as a retrospective marker of hypothalamic-pituitaryadrenal axis activity in horse foals. Vet. J., 194, 131-132.

Conard, M.A. \& Matthews, R.A. (2008). Modeling the stress process: Personality eclipses dysfunctional cognitions and workload in predicting stress. Pers. Individ. Dif., 44, 171-181.

Cook, K. V., O’Connor, C.M., Gilmour, K.M. \& Cooke, S.J. (2011). The glucocorticoid stress response is repeatable between years in a wild teleost fish. J. Comp. Physiol. A Neuroethol. Sensory, Neural, Behav. Physiol., 197, 11891196.

Cook, K.V., O’Connor, C.M., McConnachie, S.H., Gilmour, K.M. \& Cooke, S.J. (2012). Condition dependent intra-individual repeatability of stress-induced cortisol in a freshwater fish. Comp. Biochem. Physiol. Part A Mol. Integr. Physiol., 161, 337-343. 
Coppens, C.M., de Boer, S.F. \& Koolhaas, J.M. (2010). Coping styles and behavioural flexibility: towards underlying mechanisms. Philos. Trans. R. Soc. Lond. B. Biol. Sci., 365, 4021-8.

Cordeiro, E.M.G., Campbell, J.F., Phillips, T.W. \& With, K.A. (2018). Behavioral and social mechanisms behind pattern formation: an experimental study of animal movement. Landsc. Ecol., 33, 1881-1894.

Costa, J.H.C., von Keyserlingk, M.A.G. \& Weary, D.M. (2016). Invited review: Effects of group housing of dairy calves on behavior, cognition, performance, and health. J. Dairy Sci., 99, 2453-2467.

Couzin, I.D. (2006). Behavioral ecology: Social organization in fission-fusion societies. Curr. Biol.

Crino, O.L., Larkin, I. \& Phelps, S.M. (2010). Stress coping styles and singing behavior in the short-tailed singing mouse (Scotinomys teguina). Horm. Behav., $58,334-340$.

Cunningham, K. (2017). Hormonal Synchronization of Cortisol Levels and Emotional Contagion Between Human Owners and Agility Dogs. University of Nebraska Omaha.

D’Anna-Hernandez, K.L., Ross, R.G., Natvig, C.L. \& Laudenslager, M.L. (2011). Hair cortisol levels as a retrospective marker of hypothalamic-pituitary axis activity throughout pregnancy: Comparison to salivary cortisol. Physiol. Behav., 104, 348-353.

Dabbs, J.M. (1990). Salivary testosterone measurements: Reliability across hours, days, and weeks. Physiol. Behav., 48, 83-86.

Dahlgren, A., Kecklund, G., Theorell, T. \& Åkerstedt, T. (2009). Day-to-day variation in saliva cortisol-Relation with sleep, stress and self-rated health. Biol. Psychol., $82,149-155$.

Dall, S.R.X., Houston, A.I. \& McNamara, J.M. (2004). The behavioural ecology of personality: consistent individual differences from an adaptive perspective. Ecol. Lett., 7, 734-739.

Davenport, M.D., Lutz, C.K., Tiefenbacher, S., Novak, M.A. \& Meyer, J.S. (2008). A Rhesus Monkey Model of Self-Injury: Effects of Relocation Stress on Behavior and Neuroendocrine Function. Biol. Psychiatry, 63, 990-996.

Davenport, M.D., Tiefenbacher, S., Lutz, C.K., Novak, M.A. \& Meyer, J.S. (2006). Analysis of endogenous cortisol concentrations in the hair of rhesus macaques. Gen. Comp. Endocrinol., 147, 255-261.

David, J.., Gibert, P. \& Moreteau, B. (2004). Evolution of reaction norms. In: Phenotypic plasticity: functional and conceptual approaches (eds. DeWitt, T.J. \& Scheiner, S..). Oxford University Press, pp. 50-63.

Davies, N.B. (Nicholas B.., Krebs, J.R. (John R.. \& West, S.A. (2012). An introduction to behavioural ecology. Wiley-Blackwell.

DeSoto, M.C. \& Salinas, M. (2015). Neuroticism and cortisol: the importance of checking for sex differences. Psychoneuroendocrinology, 62, 174-179. 
Dettenborn, L., Tietze, A., Kirschbaum, C. \& Stalder, T. (2012). The assessment of cortisol in human hair: Associations with sociodemographic variables and potential confounders. Stress, 15, 578-588.

Dettmer, A.M., Novak, M.A., Meyer, J.S. \& Suomi, S.J. (2014). Population densitydependent hair cortisol concentrations in rhesus monkeys (Macaca mulatta). Psychoneuroendocrinology, 42, 59-67.

Dettmer, A.M., Rosenberg, K., Menard, M.T., El-Mallah, S.N., Woodward, R.A., Suomi, S.J., et al. (2017). Differential relationships between chronic hormone profiles in pregnancy and maternal investment in rhesus monkey mothers with hair loss in the neonatal period. Am. J. Primatol., 79, e22489.

DeVries, A.C., Craft, T.K.S., Glasper, E.R., Neigh, G.N. \& Alexander, J.K. (2007). Social influences on stress responses and health. Psychoneuroendocrinology, 32, 587-603.

DeVries, A.C., Glasper, E.R. \& Detillion, C.E. (2003). Social modulation of stress responses. Physiol. Behav., 79, 399-407.

DeWitt, T.J. \& Scheiner, S.. (2004). Phenotypic plasticity: functional and conceptual approaches. Oxford University Press.

DeWitt, T.J., Sih, A. \& Wilson, D.S. (1998). Costs and limits of phenotypic plasticity. Trends Ecol. Evol., 13, 77-81.

Dillon, P., Buckley, F., O’Connor, P., Hegarty, D. \& Rath, M. (2003). A comparison of different dairy cow breeds on a seasonal grass-based system of milk production. 1. Milk production, live weight, body condition score and DM intake. Livest. Prod. Sci., 83, 21-33.

Dingemanse, N.J., Kazem, A.J.N., Réale, D. \& Wright, J. (2010). Behavioural reaction norms: animal personality meets individual plasticity. Trends Ecol. Evol., 25, 8189.

Dingemanse, N.J. \& Wolf, M. (2013). Between-individual differences in behavioural plasticity within populations: causes and consequences. Anim. Behav., 85, 10311039.

Ditzen, B., Neumann, I.D., Bodenmann, G., von Dawans, B., Turner, R.A., Ehlert, U., et al. (2007). Effects of different kinds of couple interaction on cortisol and heart rate responses to stress in women. Psychoneuroendocrinology, 32, 565-574.

Donker, M. (2015). Physiological Stress, Perceived Stress, and Mathematical Performance in Primary School Children.

Doyle, R.E., Lee, C., Deiss, V., Fisher, A.D., Hinch, G.N. \& Boissy, A. (2011). Measuring judgement bias and emotional reactivity in sheep following long-term exposure to unpredictable and aversive events. Physiol. Behav., 102, 503-510.

Dronjak, S., Gavrilović, L., Filipović, D. \& Radojčić, M.B. (2004). Immobilization and cold stress affect sympatho-adrenomedullary system and pituitaryadrenocortical axis of rats exposed to long-term isolation and crowding. Physiol. Behav., 81, 409-415.

Dufty, A.M., Clobert, J. \& Møller, A.P. (2002). Hormones, developmental plasticity 
and adaptation. Trends Ecol. Evol.

Dyer, J.R.G., Croft, D.P., Morrell, L.J. \& Krause, J. (2009). Shoal composition determines foraging success in the guppy. Behav. Ecol., 20, 165-171.

Dzieweczynski, T.L., Gill, C.E. \& Perazio, C.E. (2012). Opponent familiarity influences the audience effect in male-male interactions in Siamese fighting fish. Anim. Behav., 83, 1219-1224.

Dzviti, M., Mapfumo, L. \& Muchenje, V. (2019). Relationship between saliva and blood cortisol in handled cows. Asian-Australasian J. Anim. Sci., 32, 734-741.

Edwards, D.A. \& Casto, K. V. (2013). Women's intercollegiate athletic competition: cortisol, testosterone, and the dual-hormone hypothesis as it relates to status among teammates. Horm. Behav., 64, 153-160.

Edwards, D.A. \& Kurlander, L.S. (2010). Women's intercollegiate volleyball and tennis: effects of warm-up, competition, and practice on saliva levels of cortisol and testosterone. Horm. Behav., 58, 606-613.

Edwards, D.A., Wetzel, K. \& Wyner, D.R. (2006). Intercollegiate soccer: saliva cortisol and testosterone are elevated during competition, and testosterone is related to status and social connectedness with teammates. Physiol. Behav., 87, $135-143$.

Eeva, T., Lehikoinen, E. \& Nikinmaa, M. (2003). Pollution-induced nutritional stress in birds: an experimental study of direct and indirect effects. Ecol. Appl., 13, $1242-1249$.

Eisenegger, C., Haushofer, J. \& Fehr, E. (2011). The role of testosterone in social interaction. Trends Cogn. Sci., 15, 263-271.

Ellenberg, U., Setiawan, A.N., Cree, A., Houston, D.M. \& Seddon, P.J. (2007). Elevated hormonal stress response and reduced reproductive output in Yelloweyed penguins exposed to unregulated tourism. Gen. Comp. Endocrinol., 152, $54-63$.

Ellis, T., James, J.D. \& Scott, A.P. (2005). Branchial release of free cortisol and melatonin by rainbow trout. J. Fish Biol., 67, 535-540.

Ellis, T., James, J.D., Stewart, C. \& Scott, A.P. (2004). A non-invasive stress assay based upon measurement of free cortisol released into the water by rainbow trout. J. Fish Biol., 65, 1233-1252.

Ergon, T. \& Ergon, R. (2015). When three traits make a line: Evolution of phenotypic plasticity and genetic assimilation through linear reaction norms in stochastic environments. bioRxiv, 034256.

Esposito, L., Auletta, L., Ciani, F., Pelagalli, A., Pasolini, M.P., Lamagna, B., et al. (2017). Hair cortisol levels in captive brown hare (Lepus europaeus): potential effect of sex, age, and breeding technology. Eur. J. Wildl. Res., 63, 62.

Esteban, M. \& Castaño, A. (2009). Non-invasive matrices in human biomonitoring: A review. Environ. Int.

Fairbanks, L.A., Jorgensen, M.J., Bailey, J.N., Breidenthal, S.E., Grzywa, R. \& 
Laudenslager, M.L. (2011). Heritability and genetic correlation of hair cortisol in vervet monkeys in low and higher stress environments. Psychoneuroendocrinology, 36, 1201-1208.

Fardi, S., Sauther, M.L., Cuozzo, F.P., Jacky, I.A.Y. \& Bernstein, R.M. (2018). The effect of extreme weather events on hair cortisol and body weight in a wild ringtailed lemur population (Lemur catta) in southwestern Madagascar. Am. J. Primatol., 80, e22731.

Feller, S., Vigl, M., Bergmann, M.M., Boeing, H., Kirschbaum, C. \& Stalder, T. (2014). Predictors of hair cortisol concentrations in older adults. Psychoneuroendocrinology, 39, 132-140.

Ferland, C.L. \& Schrader, L.A. (2011). Cage mate separation in pair-housed male rats evokes an acute stress corticosterone response. Neurosci. Lett., 489, 154-158.

Ferrari, S., Millot, S., Leguay, D., Chatain, B. \& Bégout, M.L. (2015). Consistency in European seabass coping styles: A life-history approach. Appl. Anim. Behav. Sci., $167,74-88$.

Fischer, E.K., Harris, R.M., Hofmann, H.A. \& Hoke, K.L. (2014). Predator exposure alters stress physiology in guppies across timescales. Horm. Behav., 65, 165-172.

Fokidis, B., Burin des Roziers, M., Sparr, R., Rogowski, C., Sweazea, K. \& Deviche, P. (2012). Unpredictable food availability induces metabolic and hormonal changes independent of food intake in a sedentary songbird. J. Exp. Biol., 215, 2920-2930.

Forbes, J.M. (1977). Interrelationships between physical and metabolic control of voluntary food intake in fattening, pregnant and lactating mature sheep: a model. Anim. Prod., 24, 91-101.

Forsman, A. (2015). Rethinking phenotypic plasticity and its consequences for individuals, populations and species. Heredity (Edinb).

Fourie, N.H. \& Bernstein, R.M. (2011). Hair cortisol levels track phylogenetic and age related differences in hypothalamic-pituitary-adrenal (HPA) axis activity in nonhuman primates. Gen. Comp. Endocrinol., 174, 150-155.

Francavilla, V.C., Vitale, F., Ciaccio, M., Bongiovanni, T., Marotta, C., Caldarella, R., et al. (2018). Use of Saliva in Alternative to Serum Sampling to Monitor Biomarkers Modifications in Professional Soccer Players. Front. Physiol., 9, 1828 .

Fürtbauer, I., Brown, M.R. \& Heistermann, M. (2020). Collective action reduces androgen responsiveness with implications for shoaling dynamics in stickleback fish. Horm. Behav., 119, 104636.

Fürtbauer, I. \& Fry, A. (2018). Social conformity in solitary crabs, Carcinus maenas, is driven by individual differences in behavioural plasticity. Anim. Behav., 135, 131-137.

Fürtbauer, I. \& Heistermann, M. (2016). Cortisol coregulation in fish. Sci. Rep., 6, 30334.

Fürtbauer, I., King, A.J. \& Heistermann, M. (2015a). Visible implant elastomer (VIE) 
tagging and simulated predation risk elicit similar physiological stress responses in three-spined stickleback Gasterosteus aculeatus. J. Fish Biol., 86, 1644-1649.

Fürtbauer, I., Pond, A., Heistermann, M. \& King, A.J. (2015b). Personality, plasticity and predation: linking endocrine and behavioural reaction norms in stickleback fish. Funct. Ecol., 29, 931-940.

Fürtbauer, I., Solman, C. \& Fry, A. (2019). Sheep wool cortisol as a retrospective measure of long-term HPA axis activity and its links to body mass. Domest. Anim. Endocrinol., 68, 39-46.

Gabriel, P.O. \& Black, J.M. (2010). Behavioural syndromes in Steller's jays: the role of time frames in the assessment of behavioural traits. Anim. Behav., 80, 689697.

Gabriel, W., Luttbeg, B., Sih, A. \& Tollrian, R. (2005). Environmental tolerance, heterogeneity, and the evolution of reversible plastic responses. Am. Nat., 166, 339-53.

Galhardo, L., Vitorino, A. \& Oliveira, R.F. (2012). Social familiarity modulates personality trait in a cichlid fish. Biol. Lett., 8, 936-938.

Gangloff, E.J., Sparkman, A.M., Holden, K.G., Corwin, C.J., Topf, M. \& Bronikowski, A.M. (2017). Geographic variation and within-individual correlations of physiological stress markers in a widespread reptile, the common garter snake (Thamnophis sirtalis). Comp. Biochem. Physiol. Part A Mol. Integr. Physiol., 205, 68-76.

Gatti, R., Antonelli, G., Prearo, M., Spinella, P., Cappellin, E. \& De Palo, E.F. (2009). Cortisol assays and diagnostic laboratory procedures in human biological fluids. Clin. Biochem.

Ghalambor, C.K., McKay, J.K., Carroll, S.P. \& Reznick, D.N. (2007). Adaptive versus non-adaptive phenotypic plasticity and the potential for contemporary adaptation in new environments. Funct. Ecol., 21, 394-407.

Ghassemi Nejad, J., Kim, B.-W., Lee, B.-H. \& Sung, K.-I. (2017). Coat and hair color: hair cortisol and serotonin levels in lactating Holstein cows under heat stress conditions. Anim. Sci. J., 88, 190-194.

Ghassemi Nejad, J., Lohakare, J.D., Son, J.K., Kwon, E.G., West, J.W. \& Sung, K.I. (2014). Wool cortisol is a better indicator of stress than blood cortisol in ewes exposed to heat stress and water restriction. animal, 8, 128-132.

Gianoli, E. \& Valladares, F. (2012). Studying phenotypic plasticity: the advantages of a broad approach. Biol. J. Linn. Soc., 105, 1-7.

Gidlow, C.J., Randall, J., Gillman, J., Silk, S. \& Jones, M. V. (2016). Hair cortisol and self-reported stress in healthy, working adults. Psychoneuroendocrinology, 63, 163-169.

Glenk, L.M., Kothgassner, O.D., Stetina, B.U., Palme, R., Kepplinger, B. \& Baran, H. (2014). Salivary cortisol and behavior in therapy dogs during animal-assisted interventions: A pilot study. J. Vet. Behav. Clin. Appl. Res., 9, 98-106.

Goldberg, L.R. (1990). Personality processes and individual differences; an alternative 
"description of personality": the Big-Five factor structure. J. Personal. Psychol., $59,1216-1229$.

Goldberg, L.R. (1999). A broad-bandwidth, public domain, personality inventory measuring the lower-level of several five-factor models. In: Personality Psychology in Europe. pp. 7-28.

Gordon, M.K., Peloso, E., Auker, A. \& Dozier, M. (2005). Effect of flavored beverage crystals on salivary cortisol enzyme-immunoreactive assay measurements. Dev. Psychobiol., 47, 189-195.

Grant, K.S., Worlein, J.M., Meyer, J.S., Novak, M.A., Kroeker, R., Rosenberg, K., et al. (2017). A longitudinal study of hair cortisol concentrations in Macaca nemestrina mothers and infants. Am. J. Primatol., 79, e22591.

Griffiths, S.W. \& Magurran, A.E. (1997). Familiarity in schooling fish: how long does it take to acquire? Anim. Behav., 53, 945-949.

Grigg, E.K., Marie Nibblett, B., Robinson, J.Q. \& Smits, J.E. (2017). Evaluating pair versus solitary housing in kennelled domestic dogs (Canis familiaris) using behaviour and hair cortisol: A pilot study. Vet. Rec. Open, 4, e000193.

Gröschl, M. (2009). The physiological role of hormones in saliva. BioEssays, 31, 843 852.

Grutter, A.S. \& Pankhurst, N.W. (2000). The effects of capture, handling, confinement and ectoparasite load on plasma levels of cortisol, glucose and lactate in the coral reef fish Hemigymnus melapterus. J. Fish Biol., 57, 391-401.

Guayasamin, O.L., Couzin, I.D. \& Miller, N.Y. (2017). Behavioural plasticity across social contexts is regulated by the directionality of inter-individual differences. Behav. Processes, 141, 196-204.

Guimont, F.S. \& Wynne-Edwards, K.E. (2006). Individual variation in cortisol responses to acute "on-back" restraint in an outbred hamster. Horm. Behav., 50, 252-260.

Guindre-Parker, S. (2018). The Evolutionary Endocrinology of Circulating Glucocorticoids in Free-Living Vertebrates: Recent Advances and Future Directions across Scales of Study. Integr. Comp. Biol., 58, 814-825.

Guindre-Parker, S. (2020). Individual variation in glucocorticoid plasticity: considerations \& future directions. Integr. Comp. Biol.

Guindre-Parker, S., McAdam, A.G., Van Kesteren, F., Palme, R., Boonstra, R., Boutin, S., et al. (2019). Individual variation in phenotypic plasticity of the stress axis. Biol. Lett., 15.

Hahlbeck, E., Katsiadaki, I., Mayer, I., Adolfsson-Erici, M., James, J. \& Bengtsson, B.-E. (2004). The juvenile three-spined stickleback - model organism for the study of estrogenic and androgenic endocrine disruption in laboratory and field. Aquat. Toxicol. Elsevier B.V.

Hamel, A.F., Lutz, C.K., Coleman, K., Worlein, J.M., Peterson, E.J., Rosenberg, K.L., et al. (2017). Responses to the Human Intruder Test are related to hair cortisol phenotype and sex in rhesus macaques ( Macaca mulatta ). Am. J. Primatol., 79, 
e22526.

Hamel, A.F., Meyer, J.S., Henchey, E., Dettmer, A.M., Suomi, S.J. \& Novak, M.A. (2011). Effects of shampoo and water washing on hair cortisol concentrations. Clin. Chim. Acta, 412, 382-385.

Hamilton, L.D., Carré, J.M., Mehta, P.H., Olmstead, N. \& Whitaker, J.D. (2015). Social neuroendocrinology of status: a review and future directions. Adapt. Hum. Behav. Physiol., 1, 202-230.

Harman, S.M., Metter, E.J., Tobin, J.D., Pearson, J. \& Blackman, M.R. (2001). Longitudinal effects of aging on serum total and free testosterone levels in healthy men. J. Clin. Endocrinol. Metab., 86, 724-731.

Hau, M. \& Goymann, W. (2015). Endocrine mechanisms, behavioral phenotypes and plasticity: known relationships and open questions. Front. Zool., 12, S7.

Heimbürge, S., Kanitz, E. \& Otten, W. (2018). The use of hair cortisol for the assessment of stress in animals. Gen. Comp. Endocrinol.

Heimbürge, S., Kanitz, E., Tuchscherer, A. \& Otten, W. (2020). Within a hair's breadth - Factors influencing hair cortisol levels in pigs and cattle. Gen. Comp. Endocrinol., 288, 113359.

Heintz, M.R., Santymire, R.M., Parr, L.A. \& Lonsdorf, E. V. (2011). Validation of a cortisol enzyme immunoassay and characterization of salivary cortisol circadian rhythm in chimpanzees (Pan troglodytes). Am. J. Primatol., 73, 903-908.

Hellhammer, D.H., Wüst, S. \& Kudielka, B.M. (2009). Salivary cortisol as a biomarker in stress research. Psychoneuroendocrinology, 34, 163-171.

Hellhammer, J. \& Schubert, M. (2012). The physiological response to Trier Social Stress Test relates to subjective measures of stress during but not before or after the test. Psychoneuroendocrinology, 37, 119-124.

Hennessy, M.B., Hornschuh, G., Kaiser, S. \& Sachser, N. (2006). Cortisol responses and social buffering: A study throughout the life span. Horm. Behav., 49, 383390.

Hennessy, M.B., Kaiser, S. \& Sachser, N. (2009). Social buffering of the stress response: Diversity, mechanisms, and functions. Front. Neuroendocrinol., 30, 470-482.

Herring, G., Cook, M.I., Gawlik, D.E. \& Call, E.M. (2011). Food availability is expressed through physiological stress indicators in nestling white ibis: a food supplementation experiment. Funct. Ecol., 25, 682-690.

Hetts, S., Derrell Clark, J., Calpin, J.P., Arnold, C.E. \& Mateo, J.M. (1992). Influence of housing conditions on beagle behaviour. Appl. Anim. Behav. Sci., 34, 137-155.

Hicks, T.A., McGlone, J.J., Whisnant, C.S., Kattesh, H.G. \& Norman, R.L. (1998). Behavioral, endocrine, immune, and performance measures for pigs exposed to acute stress. J. Anim. Sci., 76, 474.

Hill, E.M., Billington, R. \& Krägeloh, C. (2013). The cortisol awakening response and the big five personality dimensions. Pers. Individ. Dif., 55, 600-605. 
Hodes, A., Lodish, M.B., Tirosh, A., Meyer, J., Belyavskaya, E., Lyssikatos, C., et al. (2017). Hair cortisol in the evaluation of Cushing syndrome. Endocrine, 56, 164174.

Hoffman, M.C., D’Anna-Hernandez, K., Benitez, P., Ross, R.G. \& Laudenslager, M.L. (2017). Cortisol during human fetal life: Characterization of a method for processing small quantities of newborn hair from 26 to 42 weeks gestation. Dev. Psychobiol., 59, 123-127.

Hofman, L.F. (2001). Human saliva as a diagnostic specimen. In: Journal of Nutrition. American Institute of Nutrition, pp. 1621-1625.

van Holland, B.J., Frings-Dresen, M.H.W. \& Sluiter, J.K. (2012). Measuring shortterm and long-term physiological stress effects by cortisol reactivity in saliva and hair. Int. Arch. Occup. Environ. Health, 85, 849-852.

Huntingford, F.A. \& Ruiz-Gomez, M.L. (2009). Three-spined sticklebacks Gasterosteus aculeatus as a model for exploring behavioural biology. J. Fish Biol., 75, 1943-1976.

Van Jaarsveld, A. \& Skinner, J. (1992). Adrenocorticol responsiveness to immobilization stress in spotted hyenas (Crocuta crocuta). Comp. Biochem. Physiol., 103, 73-79.

Jennifer Morton, A. \& Howland, D.S. (2013). Large genetic animal models of huntington's disease. J. Huntingtons. Dis., 2, 3-19.

Jensen, M.B., Munksgaard, L., Mogensen, L. \& Krohn, C.C. (1999). Effects of housing in different social environments on Open-Field and social responses of female dairy calves. Acta Agric. Scand. A Anim. Sci., 49, 113-120.

Jogems-Kosterman, B.J.M., de Knijff, D.W.W., Kusters, R. \& van Hoof, J.J.M. (2007). Basal cortisol and DHEA levels in women with borderline personality disorder. J. Psychiatr. Res., 41, 1019-1026.

Jolles, J.W., Aaron Taylor, B. \& Manica, A. (2016). Recent social conditions affect boldness repeatability in individual sticklebacks. Anim. Behav., 112, 139-145.

Jolles, J.W., Boogert, N.J., Sridhar, V.H., Couzin, I.D. \& Manica, A. (2017). Consistent Individual Differences Drive Collective Behavior and Group Functioning of Schooling Fish. Curr. Biol., 27, 2862-2868.e7.

Jolles, J.W., Fleetwood-Wilson, A., Nakayama, S., Stumpe, M.C., Johnstone, R.A. \& Manica, A. (2014). The role of previous social experience on risk-taking and leadership in three-spined sticklebacks. Behav. Ecol., 25, 1395-1401.

Jolles, J.W., Laskowski, K.L., Boogert, N.J. \& Manica, A. (2018). Repeatable group differences in the collective behaviour of stickleback shoals across ecological contexts. Proc. R. Soc. B Biol. Sci., 285, 20172629.

Kalra, S., Einarson, A., Karaskov, T., Van Uum, S. \& Koren, G. (2007). The relationship between stress and hair cortisol in healthy pregnant women. Clin. Investig. Med., 30.

Karlén, J., Ludvigsson, J., Frostell, A., Theodorsson, E. \& Faresjö, T. (2011). Cortisol in hair measured in young adults - a biomarker of major life stressors? BMC Clin. 
Pathol., 11, 12.

Keck, V.A., Edgerton, D.S., Hajizadeh, S., Swift, L.L., Dupont, W.D., Lawrence, C., et al. (2015). Effects of Habitat Complexity on Pair-Housed Zebrafish. J. Am. Assoc. Lab. Anim. Sci., 54, 378-383.

Keller, B.A., Finger, J.-S., Gruber, S.H., Abel, D.C. \& Guttridge, T.L. (2017). The effects of familiarity on the social interactions of juvenile lemon sharks (Negaprion brevirostris). J. Exp. Mar. Bio. Ecol., 489, 24-31.

Kelly, C. \& Price, T.D. (2005). Correcting for regression to the mean in behavior and ecology. Am. Nat., 166, 700-707.

Kenagy, G.J. \& Place, N.J. (2000). Seasonal changes in plasma glucocorticosteroids of free-living female yellow-pine chipmunks: Effects of reproduction and capture and handling. Gen. Comp. Endocrinol., 117, 189-199.

Kendrick, K.M., Da Costa, A.P., Leigh, A.E., Hinton, M.R. \& Peirce, J.W. (2001). Sheep don't forget a face. Nature, 414, 165-166.

Ketay, S., Welker, K.M. \& Slatcher, R.B. (2017). The roles of testosterone and cortisol in friendship formation. Psychoneuroendocrinology, 76, 88-96.

Khalfa, S., Bella, S.D., Roy, M., Peretz, I. \& Lupien, S.J. (2003). Effects of relaxing music on salivary cortisol level after psychological stress. Ann. N. Y. Acad. Sci., 999, 374-376.

King, A.J., Williams, L.J. \& Mettke-Hofmann, C. (2015). The effects of social conformity on Gouldian finch personality. Anim. Behav., 99, 25-31.

Kirschbaum, C., Bartussek, D. \& Strasburger, C.J. (1992). Cortisol responses to psychological stress and correlations with personality traits. Pers. Individ. Dif., $13,1353-1357$.

Kirschbaum, C., Pirke, K.M. \& Hellhammer, D.H. (1993). The 'Trier Social Stress Test'--a tool for investigating psychobiological stress responses in a laboratory setting. Neuropsychobiology, 28, 76-81.

Kirschbaum, C., Tietze, A., Skoluda, N. \& Dettenborn, L. (2009). Hair as a retrospective calendar of cortisol production-Increased cortisol incorporation into hair in the third trimester of pregnancy. Psychoneuroendocrinology, 34, 32-37.

Kitaysky, A., Piatt, J. \& Wingfield, J. (2007). Stress hormones link food availability and population processes in seabirds. Mar. Ecol. Prog. Ser., 352, 245-258.

Kitaysky, A.S., Piatt, J.F., Wingfield, J.C. \& Romano, M. (1999). The adrenocortical stress-response of Black-legged Kittiwake chicks in relation to dietary restrictions. J. Comp. Physiol. B Biochem. Syst. Environ. Physiol., 169, 303-310.

Kobayashi, H. \& Miyazaki, Y. (2015). Distribution characteristics of salivary cortisol measurements in a healthy young male population. J. Physiol. Anthropol., 34.

Koolhaas, J.., Korte, S.., De Boer, S.., Van Der Vegt, B.., Van Reenen, C.., Hopster, H., et al. (1999). Coping styles in animals: current status in behavior and stressphysiology. Neurosci. Biobehav. Rev., 23, 925-935.

Koolhaas, J.M., de Boer, S.F., Coppens, C.M. \& Buwalda, B. (2010). 
Neuroendocrinology of coping styles: Towards understanding the biology of individual variation. Front. Neuroendocrinol., 31, 307-321.

Koren, L., Whiteside, D., Fahlman, Å., Ruckstuhl, K., Kutz, S., Checkley, S., et al. (2012). Cortisol and corticosterone independence in cortisol-dominant wildlife. Gen. Comp. Endocrinol., 177, 113-119.

Kornienko, O., Clemans, K.H., Out, D. \& Granger, D.A. (2014). Hormones, behavior, and social network analysis: Exploring associations between cortisol, testosterone, and network structure. Horm. Behav., 66, 534-544.

Kornienko, O., Schaefer, D.R., Weren, S., Hill, G.W. \& Granger, D.A. (2016). Cortisol and testosterone associations with social network dynamics. Horm. Behav., 80, 92-102.

Koski, S.E. \& Burkart, J.M. (2015). Common marmosets show social plasticity and group-level similarity in personality. Sci. Rep., 5, 8878.

Kristiansen, T.S. \& Fernö, A. (2007). Individual behaviour and growth of halibut (Hippoglossus hippoglossus L.) fed sinking and floating feed: Evidence of different coping styles. Appl. Anim. Behav. Sci., 104, 236-250.

Kroshko, T., Kapronczai, L., Cattet, M.R.L., Macbeth, B.J., Stenhouse, G.B., Obbard, M.E., et al. (2017). Comparison of methanol and isopropanol as wash solvents for determination of hair cortisol concentration in grizzly bears and polar bears. MethodsX, 4, 68-75.

Kruuk, H. \& Parish, T. (1985). Food, food availability and weight of badgers (Meles meles) in relation to agricultural changes. J. Appl. Ecol., 22, 705.

Kudielka, B.M., Hellhammer, D.H. \& Wüst, S. (2009). Why do we respond so differently? Reviewing determinants of human salivary cortisol responses to challenge. Psychoneuroendocrinology, 34, 2-18.

Kudielka, B.M. \& Kirschbaum, C. (2004). Sex differences in HPA axis responses to stress: a review.

Kurvers, R.H.J.M., Adamczyk, V.M.A.P., Kraus, R.H.S., Hoffman, J.I., van Wieren, S.E., van der Jeugd, H.P., et al. (2013). Contrasting context dependence of familiarity and kinship in animal social networks. Anim. Behav., 86, 993-1001.

Laland, K.N. \& Williams, K. (1998). Social transmission of maladaptive information in the guppy. Behav. Ecol., 9, 493-499.

Laskowski, K.L. \& Bell, A.M. (2013). Competition avoidance drives individual differences in response to a changing food resource in sticklebacks. Ecol. Lett., $16,746-753$.

Laudenslager, M.L., Jorgensen, M.J. \& Fairbanks, L.A. (2012). Developmental patterns of hair cortisol in male and female nonhuman primates: Lower hair cortisol levels in vervet males emerge at puberty. Psychoneuroendocrinology, 37, 1736-1739.

LeBlanc, J. \& Ducharme, M.B. (2005). Influence of personality traits on plasma levels of cortisol and cholesterol. Physiol. Behav., 84, 677-680. 
Levine, A., Zagoory-Sharon, O., Feldman, R., Lewis, J.G. \& Weller, A. (2007). Measuring cortisol in human psychobiological studies. Physiol. Behav., 90, 4353.

Lewis, J.G. (2006). Steroid analysis in saliva: an overview. Clin. Biochem. Rev., 27, 139-46.

Liebgold, E.B. \& Dibble, C.J. (2011). Better the devil you know: familiarity affects foraging activity of red-backed salamanders (Plethodon cinereus). Anim. Behav., 82, 1059-1066.

Liening, S.H., Stanton, S.J., Saini, E.K. \& Schultheiss, O.C. (2010). Salivary testosterone, cortisol, and progesterone: Two-week stability, interhormone correlations, and effects of time of day, menstrual cycle, and oral contraceptive use on steroid hormone levels. Physiol. Behav., 99, 8-16.

Lippi, G., Dipalo, M., Buonocore, R., Gnocchi, C., Aloe, R. \& Delsignore, R. (2016). Analytical Evaluation of Free Testosterone and Cortisol Immunoassays in Saliva as a Reliable Alternative to Serum in Sports Medicine. J. Clin. Lab. Anal., 30, 732-735.

Littin, K.E. \& Cockrem, J.F. (2001). Individual variation in corticosterone secretion in laying hens. Br. Poult. Sci., 42, 536-546.

Liu, S., Rovine, M.J., Cousino Klein, L. \& Almeida, D.M. (2013). Synchrony of diurnal cortisol pattern in couples. J. Fam. Psychol., 27, 579-588.

Lockwood, S.A., Kattesh, H.G., Rhinehart, J.D., Strickland, L.G., Krawczel, P.D., Wilkerson, J.B., et al. (2017). Relationships among temperament, acute and chronic cortisol and testosterone concentrations, and breeding soundness during performance testing of Angus bulls. Theriogenology, 89, 140-145.

Lovallo, W.R., Farag, N.H., Vincent, A.S., Thomas, T.L. \& Wilson, M.F. (2006). Cortisol responses to mental stress, exercise, and meals following caffeine intake in men and women. Pharmacol. Biochem. Behav., 83, 441-447.

Lowry, H., Lill, A. \& Wong, B.B.M. (2013). Behavioural responses of wildlife to urban environments. Biol. Rev., 88, 537-549.

Lucon-Xiccato, T., Mazzoldi, C. \& Griggio, M. (2017). Sex composition modulates the effects of familiarity in new environment. Behav. Processes, 140, 133-138.

Lush, L., Wilson, R.P., Holton, M.D., Hopkins, P., Marsden, K.A., Chadwick, D.R., et al. (2018). Classification of sheep urination events using accelerometers to aid improved measurements of livestock contributions to nitrous oxide emissions. Comput. Electron. Agric., 150, 170-177.

Lyons, D.M., Price, E.O. \& Moberg, G.P. (1993). Social grouping tendencies and separation-induced distress in juvenile sheep and goats. Dev. Psychobiol., 26, 251-259.

Macbeth, B.J., Cattet, M.R.L., Obbard, M.E., Middel, K. \& Janz, D.M. (2012). Evaluation of hair cortisol concentration as a biomarker of long-term stress in free-ranging polar bears. Wildl. Soc. Bull., 36, 747-758.

Madliger, C.L. \& Love, O.P. (2016). Employing individual measures of baseline 
glucocorticoids as population-level conservation biomarkers: considering withinindividual variation in a breeding passerine. Conserv. Physiol., 4, cow048.

Maestripieri, D. \& Hoffman, C.L. (2011). Chronic stress, allostatic load, and aging in nonhuman primates. Dev. Psychopathol., 23, 1187-1195.

Manenschijn, L., Koper, J.W., Lamberts, S.W.J. \& Van Rossum, E.F.C. (2011a). Evaluation of a method to measure long term cortisol levels. Steroids, 76, 10321036.

Manenschijn, L., van Kruysbergen, R.G.P.M., de Jong, F.H., Koper, J.W. \& van Rossum, E.F.C. (2011b). Shift work at young age is associated with elevated long-term cortisol levels and body mass index. J. Clin. Endocrinol. Metab., 96, E1862-E1865.

Manning, A. \& Dawkins, M.S. (1998). Social organization. In: An Introduction to Animal Behaviour. Cambridge University Press, pp. 355-404.

Marchand, A., Durand, P. \& Lupien, S. (2013). Work hours and cortisol variation from non-working to working days. Int. Arch. Occup. Environ. Health, 86, 553-559.

Marco, E.M., Rapino, C., Caprioli, A., Borsini, F., Maccarrone, M. \& Laviola, G. (2011). Social encounter with a novel partner in adolescent rats: Activation of the central endocannabinoid system. Behav. Brain Res., 220, 140-145.

Marras, S., Killen, S.S., Claireaux, G., Domenici, P. \& McKenzie, D.J. (2011). Behavioural and kinematic components of the fast-start escape response in fish: individual variation and temporal repeatability. J. Exp. Biol., 214, 3102-10.

Martins, C.I.M., Schrama, J.W. \& Verreth, J.A.J. (2006). The relationship between individual differences in feed efficiency and stress response in African catfish Clarias gariepinus. Aquaculture, 256, 588-595.

Martins, C.I.M., Silva, P.I.M., Conceição, L.E.C., Costas, B., Höglund, E., Øverli, Ø., et al. (2011). Linking fearfulness and coping styles in fish. PLoS One, 6.

Matsuda, S., Yamaguchi, T., Okada, K., Gotouda, A. \& Mikami, S. (2012). Day-today variations in salivary cortisol measurements. J. Prosthodont. Res., 56, 37-41.

Matsunaga, W. \& Watanabe, E. (2010). Habituation of medaka (Oryzias latipes) demonstrated by open-field testing. Behav. Processes, 85, 142-150.

McDonald, N.D., Rands, S.A., Hill, F., Elder, C. \& Ioannou, C.C. (2016). Consensus and experience trump leadership, suppressing individual personality during social foraging. Sci. Adv., 2, e1600892.

McEwen, B.S. \& Wingfield, J.C. (2010). What is in a name? Integrating homeostasis, allostasis and stress. Horm. Behav.

McKinnon, J.S. \& McPhail, J.D. (1996). Male aggression and colour in divergent populations of the threespine stickleback: Experiments with animations. Can. J. Zool., 74, 1727-1733.

McQuillan, H.J., Lokman, P.M. \& Young, G. (2003). Effects of sex steroids, sex, and sexual maturity on cortisol production: An in vitro comparison of chinook salmon and rainbow trout interrenals. Gen. Comp. Endocrinol., 133, 154-163. 
Meagher, R.K., Daros, R.R., Costa, J.H.C., von Keyserlingk, M.A.G., Hötzel, M.J. \& Weary, D.M. (2015). Effects of Degree and Timing of Social Housing on Reversal Learning and Response to Novel Objects in Dairy Calves. PLoS One, $10, \mathrm{e} 0132828$.

Meka, J.M. \& McCormick, S.D. (2005). Physiological response of wild rainbow trout to angling: Impact of angling duration, fish size, body condition, and temperature. Fish. Res., 72, 311-322.

Mertens, P.A. \& Unshelm, J. (1996). Effects of group and individual housing on the behavior of kennelled dogs in animal shelters. Anthrozoos, 9, 40-51.

Meyer, J.S. \& Novak, M.A. (2012). Minireview: Hair Cortisol: A Novel Biomarker of Hypothalamic-Pituitary-Adrenocortical Activity. Endocrinology, 153, 41204127.

Miles, M.C., Vitousek, M.N., Husak, J.F., Johnson, M.A., Martin, L.B., Taff, C.C., et al. (2018). Standing Variation and the Capacity for Change: Are Endocrine Phenotypes More Variable Than Other Traits? Integr. Comp. Biol., 58, 751-762.

Miner, B.G., Sultan, S.E., Morgan, S.G., Padilla, D.K. \& Relyea, R.A. (2005). Ecological consequences of phenotypic plasticity. Trends Ecol. Evol.

Miranda-de la Lama, G.C., Villarroel, M. \& María, G.A. (2012). Behavioural and physiological profiles following exposure to novel environment and social mixing in lambs. Small Rumin. Res., 103, 158-163.

Modlmeier, A.P., Liebmann, J.E. \& Foitzik, S. (2012). Diverse societies are more productive: a lesson from ants. Proceedings. Biol. Sci., 279, 2142-50.

Mondelli, V., Pariante, C.M., Navari, S., Aas, M., D’Albenzio, A., Di Forti, M., et al. (2010). Higher cortisol levels are associated with smaller left hippocampal volume in first-episode psychosis. Schizophr. Res., 119, 75-78.

Monteiro, L.S. (1975). Food efficiency in relation to estimated growth of body components in cattle. Anim. Prod., 20, 315-335.

Montillo, M., Comin, A., Corazzin, M., Peric, T., Faustini, M., Veronesi, M.C., et al. (2014). The Effect of Temperature, Rainfall, and Light Conditions on Hair Cortisol Concentrations in Newborn Foals. J. Equine Vet. Sci., 34, 774-778.

Mörelius, E., Örtenstrand, A., Theodorsson, E. \& Frostell, A. (2015). A randomised trial of continuous skin-to-skin contact after preterm birth and the effects on salivary cortisol, parental stress, depression, and breastfeeding. Early Hum. Dev., 91, 63-70.

Morrell, L.J., Croft, D.P., Dyer, J.R.G., Chapman, B.B., Kelley, J.L., Laland, K.N., et al. (2008). Association patterns and foraging behaviour in natural and artificial guppy shoals. Anim. Behav., 76, 855-864.

Morton, A.J. \& Avanzo, L. (2011). Executive Decision-Making in the Domestic Sheep. PLoS One, 6, e15752.

Moya, D., Schwartzkopf-Genswein, K.S. \& Veira, D.M. (2013). Standardization of a non-invasive methodology to measure cortisol in hair of beef cattle. Livest. Sci., $158,138-144$. 
Müller, C., Jenni-Eiermann, S., Blondel, J., Perret, P., Caro, S.P., Lambrechts, M., et al. (2006). Effect of human presence and handling on circulating corticosterone levels in breeding blue tits (Parus caeruleus). Gen. Comp. Endocrinol., 148, 163171.

Müller, C., Jenni-Eiermann, S., Blondel, J., Perret, P., Caro, S.P., Lambrechts, M.M., et al. (2007). Circulating corticosterone levels in breeding blue tits Parus caeruleus differ between island and mainland populations and between habitats. Gen. Comp. Endocrinol., 154, 128-136.

Narayan, E., Molinia, F., Christi, K., Morley, C. \& Cockrem, J. (2010). Urinary corticosterone metabolite responses to capture, and annual patterns of urinary corticosterone in wild and captive endangered Fijian ground frogs (Platymantis vitiana). Aust. J. Zool., 58, 189.

Narayan, E.J., Cockrem, J.F. \& Hero, J.-M. (2013). Repeatability of baseline corticosterone and short-term corticosterone stress responses, and their correlation with testosterone and body condition in a terrestrial breeding anuran (Platymantis vitiana). Comp. Biochem. Physiol. Part A Mol. Integr. Physiol., 165, 304-312.

Narayan, E.J., Cockrem, J.F. \& Hero, J.M. (2012a). Urinary corticosterone metabolite responses to capture and handling in two closely related species of free-living Fijian frogs. Gen. Comp. Endocrinol., 177, 55-61.

Narayan, E.J., Molinia, F.C., Cockrem, J.F. \& Hero, J.M. (2012b). Individual variation and repeatability in urinary corticosterone metabolite responses to capture in the cane toad (Rhinella marina). Gen. Comp. Endocrinol., 175, 284-289.

Nater, U.M., Hoppmann, C. \& Klumb, P.L. (2010). Neuroticism and conscientiousness are associated with cortisol diurnal profiles in adults-Role of positive and negative affect. Psychoneuroendocrinology, 35, 1573-1577.

Nejad, J.G., Kim, B.-W., Lee, B.-H., Kim, J.-Y. \& Sung, K.-I. (2017). Effects of water addition to total mixed ration on water intake, nutrient digestibility, wool cortisol and blood indices in Corriedale ewes. Asian-Australasian J. Anim. Sci., 30, 14351441.

Nelson, R.J. (2011). An introduction to behavioural endocrinology. 4th edn. Sinauer Associates.

Nettle, D. \& Penke, L. (2010). Personality: bridging the literatures from human psychology and behavioural ecology. Philos. Trans. R. Soc. B Biol. Sci., 365, 4043-4050.

Neu, M., Laudenslager, M.L. \& Robinson, J. (2008). Coregulation in Salivary Cortisol During Maternal Holding of Premature Infants. Biol. Res. Nurs., 10, 226-240.

Noh, S., Everman, E.R., Berger, C.M. \& Morgan, T.J. (2017). Seasonal variation in basal and plastic cold tolerance: Adaptation is influenced by both long- and shortterm phenotypic plasticity. Ecol. Evol., 7, 5248-5257.

Nussey, D.H., Wilson, A.J. \& Brommer, J.E. (2007). The evolutionary ecology of individual phenotypic plasticity in wild populations. J. Evol. Biol., 20, 831-844.

O'Connor, E.A., Pottinger, T.G. \& Sneddon, L.U. (2011). The effects of acute and 
chronic hypoxia on cortisol, glucose and lactate concentrations in different populations of three-spined stickleback. Fish Physiol. Biochem., 37, 461-469.

O'Neill, S.J., Williamson, J.E., Tosetto, L. \& Brown, C. (2018). Effects of acclimatisation on behavioural repeatability in two behaviour assays of the guppy Poecilia reticulata. Behav. Ecol. Sociobiol., 72, 1-11.

Obel, C., Hedegaard, M., Henriksen, T.B., Secher, N.J., Olsen, J. \& Levine, S. (2005). Stress and salivary cortisol during pregnancy. Psychoneuroendocrinology, 30, 647-656.

Oei, N.Y.L., Elzinga, B.M., Wolf, O.T., de Ruiter, M.B., Damoiseaux, J.S., Kuijer, J.P.A., et al. (2007). Glucocorticoids Decrease Hippocampal and Prefrontal Activation during Declarative Memory Retrieval in Young Men. Brain Imaging Behav., 1, 31-41.

van Oers, K., Klunder, M. \& Drent, P.J. (2005). Context dependence of personalities: risk-taking behavior in a social and a nonsocial situation. Behav. Ecol., 16, 716723.

Oliveira, R. \& Galhardo, L. (2009). Psychological Stress and Welfare in Fish. Annu. Rev. Biomed. Sci., 11, 1-20.

Olsson, I.A.S. \& Westlund, K. (2007). More than numbers matter: The effect of social factors on behaviour and welfare of laboratory rodents and non-human primates. Appl. Anim. Behav. Sci., 103, 229-254.

Olstad, D.L., Ball, K., Wright, C., Abbott, G., Brown, E. \& Turner, A.I. (2016). Hair cortisol levels, perceived stress and body mass index in women and children living in socioeconomically disadvantaged neighborhoods: The READI study. Stress, 19, 158-167.

Oswald, L.M., Zandi, P., Nestadt, G., Potash, J.B., Kalaydjian, A.E. \& Wand, G.S. (2006). Relationship between Cortisol Responses to Stress and Personality. Neuropsychopharmacology, 31, 1583-1591.

Ouyang, J.Q., Sharp, P.J., Dawson, A., Quetting, M. \& Hau, M. (2011). Hormone levels predict individual differences in reproductive success in a passerine bird. Proceedings. Biol. Sci., 278, 2537-45.

Øverli, Ø., Korzan, W.J., Höglund, E., Winberg, S., Bollig, H., Watt, M., et al. (2004). Stress coping style predicts aggression and social dominance in rainbow trout. Horm. Behav., 45, 235-241.

Pankhurst, N.W. (2011). The endocrinology of stress in fish: An environmental perspective. Gen. Comp. Endocrinol., 170, 265-275.

Pankhurst, N.W., Wells, R.M.G. \& Carragher, J.F. (1992). Effects of stress on plasma cortisol levels and blood viscosity in blue mao mao, Scorpis violaceus (hutton), a marine teleost. Comp. Biochem. Physiol. -- Part A Physiol., 101, 335-339.

Papp, L.M., Pendry, P. \& Adam, E.K. (2009). Mother-Adolescent Physiological Synchrony in Naturalistic Settings: Within-Family Cortisol Associations and Moderators. J. Fam. Psychol., 23, 882-894.

Papp, L.M., Pendry, P., Simon, C.D. \& Adam, E.K. (2013). Spouses' Cortisol 
Associations and Moderators: Testing Physiological Synchrony and Connectedness in Everyday Life. Fam. Process, 52, 284-298.

Parent-Lamarche, A. \& Marchand, A. (2015). The moderating role of personality traits in the relationship between work and salivary cortisol: a cross-sectional study of 401 employees in 34 Canadian companies. BMC Psychol., 3, 45.

Paulsen, R.B., Wilkins, D.G., Slawson, M.H., Shaw, K. \& Rollins, D.E. (2001). Effect off four laboratory decontamination procedures on the quantitative determination of cocaine and metabolites in hair by HPLC-MS. J. Anal. Toxicol., 25, 490-496.

Pavlidis, M., Digka, N., Theodoridi, A., Campo, A., Barsakis, K., Skouradakis, G., et al. (2013). Husbandry of Zebrafish, Danio Rerio , and the Cortisol Stress Response. Zebrafish, 10, 524-531.

Pérez-Escudero, A., Vicente-Page, J., Hinz, R.C., Arganda, S. \& de Polavieja, G.G. (2014). idTracker: tracking individuals in a group by automatic identification of unmarked animals. Nat. Methods, 11, 743-748.

Peric, T., Corazzin, M., Romanzin, A., Bovolenta, S., Prandi, A., Montillo, M., et al. (2017). Cortisol and DHEA concentrations in the hair of dairy cows managed indoor or on pasture. Livest. Sci., 202, 39-43.

Pfefferle, D., Plümer, S., Burchardt, L., Treue, S. \& Gail, A. (2018). Assessment of stress responses in rhesus macaques (Macaca mulatta) to daily routine procedures in system neuroscience based on salivary cortisol concentrations. PLoS One, 13, e0190190.

Piersma, T. \& Drent, J. (2003). Phenotypic flexibility and the evolution of organismal design. Trends Ecol. Evol., 18, 228-233.

Pinnapureddy, A.R., Stayner, C., McEwan, J., Baddeley, O., Forman, J. \& Eccles, M.R. (2015). Large animal models of rare genetic disorders: Sheep as phenotypically relevant models of human genetic disease. Orphanet J. Rare Dis.

Pluess, M., Bolten, M., Pirke, K.M. \& Hellhammer, D. (2010). Maternal trait anxiety, emotional distress, and salivary cortisol in pregnancy. Biol. Psychol., 83, 169175 .

Pottinger, T.G., Cook, A., Jürgens, M.D., Sebire, M., Henrys, P.A., Katsiadaki, I., et al. (2011). Indices of stress in three-spined sticklebacks Gasterosteus aculeatus in relation to extreme weather events and exposure to wastewater effluent. J. Fish Biol., 79, 256-279.

Pottinger, T.G. \& Feuchtmayr, H. (2020). The short-term stress response of threespined sticklebacks to climate-related stressors: a mesocosm study. Hydrobiologia, 847, 3691-3703.

Poulin, R., Hammond-Tooke, C.A. \& Nakagawa, S. (2012). Parasitism and behavioural syndromes in the fish Gobiomorphus cotidianus. Behaviour, 149, 601-622.

Powell, D.J. \& Schlotz, W. (2012). Daily Life Stress and the Cortisol Awakening Response: Testing the Anticipation Hypothesis. PLoS One, 7, e52067.

Price, E., Coleman, R., Ahsmann, J., Glendewar, G., Hunt, J., Smith, T., et al. (2019). 
Individual, social, and environmental factors affecting salivary and fecal cortisol levels in captive pied tamarins (Saguinus bicolor). Am. J. Primatol., 81.

Pride, R.E. (2005). High faecal glucocorticoid levels predict mortality in ring-tailed lemurs (Lemur catta). Biol. Lett., 1, 60-63.

R Development Core Team, R. (2018). R: A language and environment for statistical computing.

Raul, J.S., Cirimele, V., Ludes, B. \& Kintz, P. (2004). Detection of physiological concentrations of cortisol and cortisone in human hair. Clin. Biochem., 37, 11051111.

Reefmann, N., Wechsler, B. \& Gygax, L. (2009). Behavioural and physiological assessment of positive and negative emotion in sheep. Anim. Behav., 78, 651659.

Remage-Healey, L., Adkins-Regan, E. \& Romero, L.M. (2003). Behavioral and adrenocortical responses to mate separation and reunion in the zebra finch. Horm. Behav., 43, 108-114.

Rensel, M.A. \& Schoech, S.J. (2011). Repeatability of baseline and stress-induced corticosterone levels across early life stages in the Florida scrub-jay (Aphelocoma coerulescens). Horm. Behav., 59, 497-502.

Restituto, P., Galofré, J.C., Gil, M.J., Mugueta, C., Santos, S., Monreal, J.I., et al. (2008). Advantage of salivary cortisol measurements in the diagnosis of glucocorticoid related disorders. Clin. Biochem., 41, 688-692.

Riek, A., Schrader, L., Zerbe, F. \& Petow, S. (2019). Comparison of cortisol concentrations in plasma and saliva in dairy cattle following ACTH stimulation. J. Dairy Res., 86, 406-409.

Rieucau, G., Morand-Ferron, J. \& Giraldeau, L.-A. (2010). Group size effect in nutmeg mannikin: between-individuals behavioral differences but same plasticity. Behav. Ecol., 21, 684-689.

Romero, L.M., Dickens, M.J. \& Cyr, N.E. (2009). The reactive scope model - A new model integrating homeostasis, allostasis, and stress. Horm. Behav.

Romero, L.M. \& Reed, J.M. (2008). Repeatability of baseline corticosterone concentrations. Gen. Comp. Endocrinol., 156, $27-33$.

Romero, L.M. \& Wikelski, M. (2001). Corticosterone levels predict survival probabilities of galápagos marine iguanas during El Niño events. Proc. Natl. Acad. Sci. U. S. A., 98, 7366-7370.

Rose, C.L., Murphy, L.B., Byard, L. \& Nikzad, K. (2002). The role of the Big Five personality factors in vigilance performance and workload. Eur. J. Pers., 16, 185200.

Rose, R.M., Kreuz, L.E., Holaday, J.W., Sulak, K.J. \& Johnson, C.E. (1972). Diurnal Variation Of Plasma Testosterone And Cortisol. J. Endocrinol., 54, 177-178.

Roth, L.S. V., Faresjö, Å., Theodorsson, E. \& Jensen, P. (2016). Hair cortisol varies with season and lifestyle and relates to human interactions in German shepherd 
dogs. Sci. Rep., 6, 19631.

De Rouck, M. \& Law, G. (2005). A comparative study of the influence of social housing on the behavior of captive tigers. Anim. Welf., 14, 229-238.

Roy, T. \& Bhat, A. (2018). Repeatability in boldness and aggression among wild zebrafish (Danio rerio) from two differing predation and flow regimes. J. Comp. Psychol., 132, 349-360.

Ruane, N.M. \& Komen, H. (2003). Measuring cortisol in the water as an indicator of stress caused by increased loading density in common carp (Cyprinus carpio). Aquaculture, 218, 685-693.

Rudolph, K.E., Sánchez, B.N., Stuart, E.A., Greenberg, B., Fujishiro, K., Wand, G.S., et al. (2016). Job strain and the cortisol diurnal cycle in MESA: Accounting for between- and within-day variability. Am. J. Epidemiol.

Ruis, M.A.W., Te Brake, J.H.A., Engel, B., Ekkel, E.D., Buist, W.G., Blokhuis, H.J., et al. (1997). The circadian rhythm of salivary cortisol in growing pigs: effects of age, gender, and stress. Physiol. Behav., 62, 623-630.

Ruiz-Gomez, M. de L., Huntingford, F.A., Øverli, Ø., Thörnqvist, P.-O. \& Höglund, E. (2011). Response to environmental change in rainbow trout selected for divergent stress coping styles. Physiol. Behav., 102, 317-322.

Ruiz-Gomez, M. de L., Kittilsen, S., Höglund, E., Huntingford, F.A., Sørensen, C., Pottinger, T.G., et al. (2008). Behavioral plasticity in rainbow trout (Oncorhynchus mykiss) with divergent coping styles: When doves become hawks. Horm. Behav., 54, 534-538.

Rukstalis, M. \& French, J.A. (2005). Vocal buffering of the stress response: exposure to conspecific vocalizations moderates urinary cortisol excretion in isolated marmosets. Horm. Behav., 47, 1-7.

Ruscio, M.G., Sweeny, T., Hazelton, J., Suppatkul, P. \& Sue Carter, C. (2007). Social environment regulates corticotropin releasing factor, corticosterone and vasopressin in juvenile prairie voles. Horm. Behav., 51, 54-61.

Russell, E., Koren, G., Rieder, M. \& Van Uum, S. (2012). Hair cortisol as a biological marker of chronic stress: Current status, future directions and unanswered questions. Psychoneuroendocrinology, 37, 589-601.

Sachser, N., Kaiser, S. \& Hennessy, M.B. (2013). Behavioural profiles are shaped by social experience: when, how and why. Philos. Trans. R. Soc. B Biol. Sci., 368, 20120344.

Salaberger, T., Millard, M., Makarem, S. El, Möstl, E., Grünberger, V., KrametterFrötscher, R., et al. (2016). Influence of external factors on hair cortisol concentrations. Gen. Comp. Endocrinol., 233, 73-78.

Samaras, A., Dimitroglou, A., Sarropoulou, E., Papaharisis, L., Kottaras, L. \& Pavlidis, M. (2016). Repeatability of cortisol stress response in the European sea bass (Dicentrarchus labrax) and transcription differences between individuals with divergent responses. Sci. Rep., 6, 1-11.

Sánchez-Muros, M.J., Villacreces, S., Miranda-de la Lama, G., de Haro, C. \& García- 
Barroso, F. (2013). Effects of chemical and handling exposure on fatty acids, oxidative stress and morphological welfare indicators in gilt-head sea bream (Sparus aurata). Fish Physiol. Biochem., 39, 581-591.

Sapolsky, R. (1994). Individual differences and the stress response. Neurosci.

Sapolsky, R.M., Romero, L.M. \& Munck, A.U. (2000). How Do Glucocorticoids Influence Stress Responses? Integrating Permissive, Suppressive, Stimulatory, and Preparative Actions*. Endocr. Rev., 21, 55-89.

Sauvé, B., Koren, G., Walsh, G., Tokmakejian, S. \& Van Uum, S.H.M. (2007). Measurement of cortisol in human hair as a biomarker of systemic exposure. Clin. Investig. Med., 30, E183-E191.

Sawyer, G., Webster, D. \& Narayan, E. (2019). Measuring wool cortisol and progesterone levels in breeding maiden Australian merino sheep (Ovis aries). PLoS One, 14, e0214734.

Saxbe, D. \& Repetti, R.L. (2010). For Better or Worse? Coregulation of Couples' Cortisol Levels and Mood States. J. Pers. Soc. Psychol., 98, 92-103.

Saxbe, D.E., Adam, E.K., Schetter, C.D., Guardino, C.M., Simon, C., McKinney, C.O., et al. (2015). Cortisol covariation within parents of young children: Moderation by relationship aggression. Psychoneuroendocrinology, 62, 121128.

Sbarra, D.A. \& Hazan, C. (2008). Coregulation, Dysregulation, Self-Regulation: An Integrative Analysis and Empirical Agenda for Understanding Adult Attachment, Separation, Loss, and Recovery. Personal. Soc. Psychol. Rev., 12, 141-167.

Schmidt, K.L., MacDougall-Shackleton, E.A., Soma, K.K. \& MacDougallShackleton, S.A. (2014). Developmental programming of the HPA and HPG axes by early-life stress in male and female song sparrows. Gen. Comp. Endocrinol., 196, 72-80.

Schmitt, T.L., St. Aubin, D.J., Schaefer, A.M. \& Dunn, J.L. (2010). Baseline, diurnal variations, and stress-induced changes of stress hormones in three captive beluga whales, Delphinapterus leucas. Mar. Mammal Sci., 26, 635-647.

Schoenemann, K.L. \& Bonier, F. (2018). Repeatability of glucocorticoid hormones in vertebrates: a meta-analysis. PeerJ, 6, e4398.

Schoenle, L.A., Zimmer, C. \& Vitousek, M.N. (2018). Understanding Context Dependence in Glucocorticoid-Fitness Relationships: The Role of the Nature of the Challenge, the Intensity and Frequency of Stressors, and Life History. Integr. Comp. Biol., 58, 777-789.

Schreck, C.. \& Tort, L. (2016). The Concept of Stress in Fish. Fish Physiol., 35, 1-34.

Schuett, W. \& Dall, S.R.X. (2009). Sex differences, social context and personality in zebra finches (Taeniopygia guttata). Anim. Behav., 77, 1041-1050.

Schumann, K., Guenther, A., Jewgenow, K. \& Trillmich, F. (2014). Animal Housing and Welfare: Effects of Housing Conditions on Body Weight and Cortisol in a Medium-Sized Rodent (Cavia aperea). J. Appl. Anim. Welf. Sci., 17, 111-124. 
Schwartz, E.B., Granger, D.A., Susman, E.J., Gunnar, M.R. \& Laird, B. (1998). Assessing salivary cortisol in studies of child development. Child Dev., 69, 15031513.

Scott, A.P. \& Ellis, T. (2007). Measurement of fish steroids in water-a review. Gen. Comp. Endocrinol.

Scott, A.P., Hirschenhauser, K., Bender, N., Oliveira, R., Earley, R.L., Sebire, M., et al. (2008). Non-invasive measurement of steroids in fish-holding water: Important considerations when applying the procedure to behaviour studies. In: Behaviour. Brill, pp. 1307-1328.

Sebire, M., Katsiadaki, I. \& Scott, A.P. (2007). Non-invasive measurement of 11ketotestosterone, cortisol and androstenedione in male three-spined stickleback (Gasterosteus aculeatus). Gen. Comp. Endocrinol., 152, 30-38.

Shams, S., Amlani, S., Buske, C., Chatterjee, D. \& Gerlai, R. (2018). Developmental social isolation affects adult behavior, social interaction, and dopamine metabolite levels in zebrafish. Dev. Psychobiol., 60, 43-56.

Sherman, G.D., Lee, J.J., Cuddy, A.J.C., Renshon, J., Oveis, C., Gross, J.J., et al. (2012). Leadership is associated with lower levels of stress. Proc. Natl. Acad. Sci. U. S. A., 109, 17903-7.

Shoal, G.D., Giancola, P.R. \& Kirillova, G.P. (2003). Salivary cortisol, personality, and aggressive behavior in adolescent boys: A 5-year longitudinal study. J. Am. Acad. Child Adolesc. Psychiatry, 42, 1101-1107.

Sih, A. (2004). A behavioral ecological view of phenotypic plasticity. In: Phenotypic plasticity: functional and conceptual approaches (eds. DeWitt, T.J. \& Scheiner, S..). Oxford University Press, pp. 112-125.

Sih, A., Ferrari, M.C.O. \& Harris, D.J. (2011). Evolution and behavioural responses to human-induced rapid environmental change. Evol. Appl., 4, 367-387.

Silva, P.I.M., Martins, C.I.M., Engrola, S., Marino, G., Øverli, Ø. \& Conceição, L.E.C. (2010). Individual differences in cortisol levels and behaviour of Senegalese sole (Solea senegalensis) juveniles: Evidence for coping styles. Appl. Anim. Behav. Sci., 124, 75-81.

Silverin, B., Arvidsson, B. \& Wingfield, J. (1997). The adrenocortical responses to stress in breeding Willow Warblers Phylloscopus trochilus in Sweden: Effects of latitude and gender. Funct. Ecol., 11, 376-384.

Siniscalchi, M., McFarlane, J.R., Kauter, K.G., Quaranta, A. \& Rogers, L.J. (2013). Cortisol levels in hair reflect behavioural reactivity of dogs to acoustic stimuli. Res. Vet. Sci., 94, 49-54.

Sladek, M.R., Doane, L.D., Luecken, L.J. \& Eisenberg, N. (2016). Perceived stress, coping, and cortisol reactivity in daily life: A study of adolescents during the first year of college. Biol. Psychol., 117, 8-15.

Smeets-Janssen, M.M.J., Roelofs, K., van Pelt, J., Spinhoven, P., Zitman, F.G., Penninx, B.W.J.H., et al. (2015). Salivary testosterone is consistently and positively associated with extraversion: results from the Netherlands study of depression and anxiety. Neuropsychobiology, 71, 76-84. 
Smith, G.T., Wingfield, J.C. \& Veit, R.R. (1994). Adrenocortical response to stress in the common diving petrel, Pelecanoides urinatrix. Physiol. Zool., 67, 526-537.

Smith, S.M. \& Vale, W.W. (2006). The role of the hypothalamic-pituitary-adrenal axis in neuroendocrine responses to stress. Dialogues Clin. Neurosci.

Smith, T.E. \& French, J.A. (1997). Psychosocial stress and urinary cortisol excretion in marmoset monkeys (Callithrix kuhli). Physiol. Behav., 62, 225-232.

Sokolowski, M.B. (2010). Social Interactions in "Simple" Model Systems. Neuron, $65,780-794$.

Sonnweber, R., Araya-Ajoy, Y.G., Behringer, V., Deschner, T., Tkaczynski, P., Fedurek, P., et al. (2018). Circadian rhythms of urinary cortisol levels vary between individuals in wild male chimpanzees: A reaction norm approach. Front. Ecol. Evol., 6, 85.

van der Staay, F.J., van Zutphen, J.A., de Ridder, M.M. \& Nordquist, R.E. (2017). Effects of environmental enrichment on decision-making behavior in pigs. Appl. Anim. Behav. Sci., 194, 14-23.

Stalder, T. \& Kirschbaum, C. (2012). Analysis of cortisol in hair - State of the art and future directions. Brain. Behav. Immun., 26, 1019-1029.

Stamp, E., Crust, L., Swann, C., Perry, J., Clough, P. \& Marchant, D. (2015). Relationships between mental toughness and psychological wellbeing in undergraduate students. Pers. Individ. Dif., 75, 170-174.

Stamps, J. \& Groothuis, T.G.G. (2010). The development of animal personality: relevance, concepts and perspectives. Biol. Rev., 85, 301-325.

Stawski, R.S., Cichy, K.E., Piazza, J.R. \& Almeida, D.M. (2013). Associations among daily stressors and salivary cortisol: Findings from the National Study of Daily Experiences. Psychoneuroendocrinology, 38, 2654-2665.

Stedman, J.M., Hallinger, K.K., Winkler, D.W. \& Vitousek, M.N. (2017). Heritable variation in circulating glucocorticoids and endocrine flexibility in a free-living songbird. J. Evol. Biol., 30, 1724-1735.

Stephens, M.A.C. \& Wand, G. (2012). Stress and the HPA axis: Role of glucocorticoids in alcohol dependence. Alcohol Res. Curr. Rev.

Steptoe, A., Easterlin, E. \& Kirschbaum, C. (2017). Conscientiousness, hair cortisol concentration, and health behaviour in older men and women. Psychoneuroendocrinology, 86, 122-127.

Sterelny, K. (2017). Humans as model organisms. Proc. R. Soc. B Biol. Sci., 284, 20172115.

Stins, J.F., Roelofs, K., Villan, J., Kooijman, K., Hagenaars, M.A. \& Beek, P.J. (2011). Walk to me when I smile, step back when I'm angry: emotional faces modulate whole-body approach-avoidance behaviors. Exp. Brain Res., 212, 603-611.

Strandburg-Peshkin, A., Papageorgiou, D., Crofoot, M.C. \& Farine, D.R. (2018). Inferring influence and leadership in moving animal groups. Philos. Trans. $R$. Soc. B Biol. Sci., 373, 20170006. 
Strum, S.C. (1991). Weight and age in wild olive baboons. Am. J. Primatol., 25, 219 237.

Stubsjøen, S.M., Bohlin, J., Dahl, E., Knappe-Poindecker, M., Fjeldaas, T., Lepschy, M., et al. (2015). Assessment of chronic stress in sheep (part I): The use of cortisol and cortisone in hair as non-invasive biological markers. Small Rumin. Res., 132, $25-31$.

Sue Carter, C., Courtney Devries, A. \& Getz, L.L. (1995). Physiological substrates of mammalian monogamy: The prairie vole model. Neurosci. Biobehav. Rev., 19, 303-314.

Swelum, A.A.A., Ayadi, M., Alhidary, I., Alowaimer, A. \& Abouheif, M. (2017). The relationships between body fatness, leptin, testosterone, and reproductive performance in ram lambs as affected by level and frequency of feeding. Theriogenology, 89, 79-85.

Taff, C.C., Schoenle, L.A. \& Vitousek, M.N. (2018). The repeatability of glucocorticoids: A review and meta-analysis. Gen. Comp. Endocrinol., 260, 136145 .

Taff, C.C. \& Vitousek, M.N. (2016). Endocrine flexibility: optimizing phenotypes in a dynamic world? Trends Ecol. Evol., 31, 476-488.

Tarullo, A.R., St. John, A.M. \& Meyer, J.S. (2017). Chronic stress in the mother-infant dyad: Maternal hair cortisol, infant salivary cortisol and interactional synchrony. Infant Behav. Dev., 47, 92-102.

Taylor, M.K. \& Cooke, S.J. (2014). Repeatability of movement behaviour in a wild salmonid revealed by telemetry. J. Fish Biol., 84, 1240-1246.

Taylor, S.C.S., Moore, A.J. \& Thiessen, R.B. (1986). Voluntary food intake in relation to body weight among British breeds of cattle. Anim. Prod., 42, 11-18.

Teruhisa, U., Ryoji, H., Taisuke, I., Tatsuya, S., Fumihiro, M. \& Tatsuo, S. (1981). Use of saliva for monitoring unbound free cortisol levels in serum. Clin. Chim. Acta, 110, 245-253.

Terwissen, C. V., Mastromonaco, G.F. \& Murray, D.L. (2013). Influence of adrenocorticotrophin hormone challenge and external factors (age, sex, and body region) on hair cortisol concentration in Canada lynx (Lynx canadensis). Gen. Comp. Endocrinol., 194, 162-167.

Thomas, M. V., Branscum, A., Miller, C.S., Ebersole, J., Al-Sabbagh, M. \& Schuster, J.L. (2009). Within-Subject Variability in Repeated Measures of Salivary Analytes in Healthy Adults. J. Periodontol., 80, 1146-1153.

Thomas, M.L. \& Simmons, L.W. (2011). Short-term phenotypic plasticity in longchain cuticular hydrocarbons. Proc. R. Soc. B Biol. Sci., 278, 3123-3128.

Thornton, R.F., Hood, R.L., Jones, P.N. \& Re, V.M. (1979). Compensatory growth in sheep. Aust. J. Agric. Res., 30, 135-151.

Thünken, T., Hesse, S., Bakker, T.C.M. \& Baldauf, S.A. (2016). Benefits of kin shoaling in a cichlid fish: familiar and related juveniles show better growth. Behav. Ecol., 27, 419-425. 
Tilbrook, A. (2000). Effects of stress on reproduction in non-rodent mammals: the role of glucocorticoids and sex differences. Rev. Reprod., 5, 105-113.

Tinghitella, R.M., Lehto, W.R. \& Lierheimer, V.F. (2018). Color and behavior differently predict competitive outcomes for divergent stickleback color morphs. Curr. Zool., 64, 115-123.

Tkaczynski, P.J., Ross, C., Lehmann, J., Mouna, M., Majolo, B. \& MacLarnon, A. (2019). Repeatable glucocorticoid expression is associated with behavioural syndromes in males but not females in a wild primate. R. Soc. Open Sci., 6.

Trevisan, C., Montillo, M., Prandi, A., Mkupasi, E.M., Ngowi, H.A. \& Johansen, M. V. (2017). Hair cortisol and dehydroepiandrosterone concentrations in naturally Taenia solium infected pigs in Tanzania. Gen. Comp. Endocrinol., 246, 23-28.

Trumble, B.C., Cummings, D., von Rueden, C., O’Connor, K.A., Smith, E.A., Gurven, M., et al. (2012). Physical competition increases testosterone among Amazonian forager-horticulturalists: a test of the "challenge hypothesis". Proceedings. Biol. Sci., 279, 2907-12.

Tsigos, C., Kyrou, I., Kassi, E. \& Chrousos, G.P. (2000). Stress, Endocrine Physiology and Pathophysiology. Endotext. MDText.com, Inc.

Tudorache, C., Ter Braake, A., Tromp, M., Slabbekoorn, H. \& Schaaf, M.J.M. (2015). Behavioral and physiological indicators of stress coping styles in larval zebrafish. Stress, 18, 121-128.

Tudorache, C., Schaaf, M.J.M. \& Slabbekoorn, H. (2013). Covariation between behaviour and physiology indicators of coping style in zebrafish (Danio rerio). J. Endocrinol., 219, 251-258.

Urhausen, A., Kullmer, T. \& Kindermann, W. (1987). A 7-week follow-up study of the behaviour of testosterone and cortisol during the competition period in rowers. Eur. J. Appl. Physiol. Occup. Physiol., 56, 528-533.

Ursache, A., Merz, E.C., Melvin, S., Meyer, J. \& Noble, K.G. (2017). Socioeconomic status, hair cortisol and internalizing symptoms in parents and children. Psychoneuroendocrinology, 78, 142-150.

Valero, A., Magurran, A.E. \& Garcia, C.M. (2009). Guppy males distinguish between familiar and unfamiliar females of a distantly related species. Anim. Behav., 78, $441-445$.

Varlinskaya, E.I., Spear, L.P. \& Spear, N.E. (1999). Social Behavior and Social Motivation in Adolescent Rats: Role of Housing Conditions and Partner's Activity. Physiol. Behav., 67, 475-482.

Vedhara, K., Miles, J., Bennett, P., Plummer, S., Tallon, D., Brooks, E., et al. (2003). An investigation into the relationship between salivary cortisol, stress, anxiety and depression. Biol. Psychol., 62, 89-96.

Verbeek, P., Iwamoto, T. \& Murakami, N. (2008). Variable stress-responsiveness in wild type and domesticated fighting fish. Physiol. Behav., 93, 83-88.

Vermeirssen, E.L.M. \& Scott, A.P. (1996). Excretion of free and conjugated steroids in rainbow trout (Oncorhynchus mykiss): Evidence for branchial excretion of the 
maturation-inducing steroid, 17,20ß-dihydroxy-4-pregnen-3-one. Gen. Comp. Endocrinol., 101, 180-194.

Vialard-Miguel, J., Belaidi, N., Lembeye, L. \& Corcuff, J.-B. (2005). Lemon juice alters cortisol assays in saliva. Clin. Endocrinol. (Oxf)., 63, 478-479.

Visser, E.K., Ellis, A.D. \& Van Reenen, C.G. (2008). The effect of two different housing conditions on the welfare of young horses stabled for the first time. Appl. Anim. Behav. Sci., 114, 521-533.

Wallerius, S., Rosmond, R., Ljung, T., Holm, G. \& Björntorp, P. (2003). Rise in morning saliva cortisol is associated with abdominal obesity in men: A preliminary report. J. Endocrinol. Invest., 26, 616-619.

Ward, A.J.W. \& Hart, P.J.B. (2003). The effects of kin and familiarity on interactions between fish. Fish Fish., 4, 348-358.

Ward, A.J.W. \& Hart, P.J.B. (2005). Foraging benefits of shoaling with familiars may be exploited by outsiders. Anim. Behav., 69, 329-335.

Ward, A.J.W., Hart, P.J.B. \& Krause, J. (2004). The effects of habitat- and diet-based cues on association preferences in three-spined sticklebacks. Behav. Ecol., 15, 925-929.

Ward, A.J.W., Sumpter, D.J.T., Couzin, I.D., Hart, P.J.B. \& Krause, J. (2008). Quorum decision-making facilitates information transfer in fish shoals. Proc. Natl. Acad. Sci., 105, 6948-6953.

Weaver, S.J., Hynd, P.I., Ralph, C.R., Hocking Edwards, J.E., Burnard, C.L., Narayan, E., et al. (2020). Chronic elevation of plasma cortisol causes differential expression of predominating glucocorticoid in plasma, saliva, fecal, and wool matrices in sheep. Domest. Anim. Endocrinol., 74, 106503.

Webster, M.M. \& Hart, P.J.B. (2006). Subhabitat selection by foraging threespine stickleback (Gasterosteus aculeatus): previous experience and social conformity. Behav. Ecol. Sociobiol., 60, 77-86.

Webster, M.M. \& Laland, K.N. (2009). Evaluation of a non-invasive tagging system for laboratory studies using three-spined sticklebacks (Gasterosteus aculeatus). J. Fish Biol., 75, 1868-1873.

Webster, M.M. \& Ward, A.J.W. (2011). Personality and social context. Biol. Rev, 86, $759-773$.

White, L.J., Thomson, J.S., Pounder, K.C., Coleman, R.C. \& Sneddon, L.U. (2017). The impact of social context on behaviour and the recovery from welfare challenges in zebrafish, Danio rerio. Anim. Behav., 132, 189-199.

Williams, T.D. (2008). Individual variation in endocrine systems: moving beyond the "tyranny of the Golden Mean". Philos. Trans. R. Soc. Lond. B. Biol. Sci., 363, 1687-98.

Windig, J.., De Kovel, C.. \& De Jong, G. (2004). Genetics and mechanics of plasticity. In: Phenotypic plasticity: functional and conceptual approaches (eds. DeWitt, T.J. \& Scheiner, S..). Oxford University Press, pp. 31-49. 
Wingfield, J.C. (2013). The comparative biology of environmental stress: behavioural endocrinology and variation in ability to cope with novel, changing environments. Anim. Behav., 85, 1127-1133.

Wingfield, J.C. \& Kitaysky, A.S. (2002). Endocrine Responses to Unpredictable Environmental Events: Stress or Anti-Stress Hormones? Integr. Comp. Biol., 42, 600-609.

Wingfield, J.C. \& Sapolsky, R.M. (2003). Reproduction and Resistance to Stress: When and How. J. Neuroendocrinol., 15, 711-724.

Wolak, M.E., Fairbairn, D.J. \& Paulsen, Y.R. (2012). Guidelines for estimating repeatability. Methods Ecol. Evol., 3, 129-137.

Wooddell, L.J., Hamel, A.F., Murphy, A.M., Byers, K.L., Kaburu, S.S.K., Meyer, J.S., et al. (2017). Relationships between affiliative social behavior and hair cortisol concentrations in semi-free ranging rhesus monkeys. Psychoneuroendocrinology, 84, 109-115.

Woodley, S.K., Matt, K.S. \& Moore, M.C. (2000). Neuroendocrine responses in freeliving female and male lizards after aggressive interactions. Physiol. Behav., 71, 373-381.

Wosu, A.C., Valdimarsdóttir, U., Shields, A.E., Williams, D.R. \& Williams, M.A. (2013). Correlates of cortisol in human hair: Implications for epidemiologic studies on health effects of chronic stress. Ann. Epidemiol.

Yamanashi, Y., Teramoto, M., Morimura, N., Nogami, E. \& Hirata, S. (2018). Social relationship and hair cortisol level in captive male chimpanzees (Pan troglodytes). Primates, 59, 145-152.

Yom-Tov, Y. \& Geffen, E. (2011). Recent spatial and temporal changes in body size of terrestrial vertebrates: probable causes and pitfalls. Biol. Rev., 86, 531-541.

Zimmer, C. (2019). Environmental unpredictability shapes glucocorticoid regulation across populations of tree swallows. bioRxiv Evol. Biol., 2019.12.23.887075. 